\title{
Supporting Information: Assessing the Accuracy of Local Hybrid Density Functional Approximations for Molecular Response Properties
}

\author{
Christof Holzer* \\ Institute of Theoretical Solid State Physics, Karlsruhe Institute of Technology (KIT), 76131 Karlsruhe, Germany \\ Yannick J. Franzke ${ }^{\dagger}$ \\ Fachbereich Chemie, Philipps-Universität Marburg, 35032 Marburg, Germany and \\ Institute of Physical Chemistry, Karlsruhe Institute of Technology (KIT), 76131 Karlsruhe, Germany \\ Max Kehry \\ Institute of Physical Chemistry, Karlsruhe Institute of Technology (KIT), 76131 Karlsruhe, Germany \\ (Dated: April 12, 2021)
}

\footnotetext{
* C. Holzer and Y. J. Franzke contributed equally to this work.; Email for correspondence: christof.holzer@kit.edu

$\dagger$ C. Holzer and Y. J. Franzke contributed equally to this work.; Email for correspondence: yannick.franzke@ chemie.uni-marburg.de
} 


\section{CONTENTS}

S1. TD-DFT Data

\begin{tabular}{|lr}
\hline A. Excitation Energies for the Small Organic Molecule Test Set & S4
\end{tabular}

\begin{tabular}{|rr}
\hline B. Excitation Energies for the Metal-Organic Test Set & S6
\end{tabular}

\begin{tabular}{|lr}
\hline C. Optimized Structures at the B3LYP/def2-TZVPP level & S9
\end{tabular}

$\begin{array}{lr}\text { S2. } G W \text { Data } & \text { S13 }\end{array}$

\begin{tabular}{|lr}
\hline A. Kohn-Sham Eigenvalues for the GW27 and GW100 Test Sets & S21
\end{tabular}

\begin{tabular}{|rr}
\hline B. Quasi-particle Energies for the GW27 and GW100 Test Sets & S26
\end{tabular}

\begin{tabular}{lr}
\hline C. Quasi-particle Energies for the GW27 and GW100 Test Sets using evGW & S31
\end{tabular}

\begin{tabular}{lr}
\hline S3. Polarizabilities and Magnetizabilities & S36
\end{tabular}

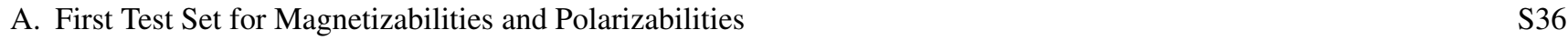

\begin{tabular}{lr}
\hline B. Second Test Set for Magnetizabilities & S40
\end{tabular}

\begin{tabular}{lr}
\hline S4. NMR Coupling Data & S42 \\
\hline
\end{tabular}

\begin{tabular}{|l|l}
\hline 1. Optimized Structures at the KT3 Level & S45 \\
\hline 2. Op &
\end{tabular}

$\begin{array}{ll}\text { 2. Optimized Structures at the BP86 Level } & \text { S47 }\end{array}$

$\begin{array}{ll}\text { 3. Optimized Structures at the PBE Level } & \text { S49 }\end{array}$

$\begin{array}{ll}\text { 4. Optimized Structures at the TPSS Level } & \text { S51 }\end{array}$

5. Optimized Structures at the BH\&HLYP Level $\quad$ S53

$\begin{array}{ll}\text { 6. Optimized Structures at the B3LYP Level } & \text { S55 }\end{array}$

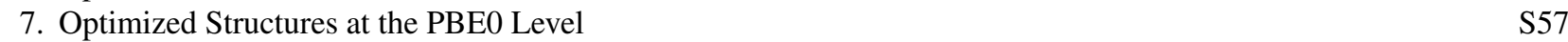

\begin{tabular}{lr}
\hline 8. Optimized Structures at the TPSSh Level & S59
\end{tabular}

9. Optimized Structures at the LC- $\omega$ PBE Level $\quad$ S61

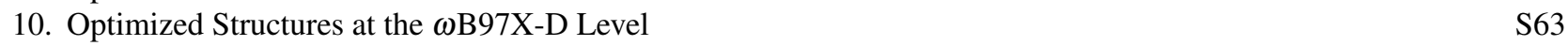

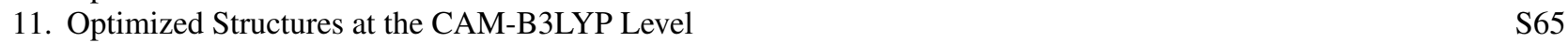

\begin{tabular}{|l|l}
\hline 12. Optimized Structures at the CAM-QPT-00 Level & S67
\end{tabular}

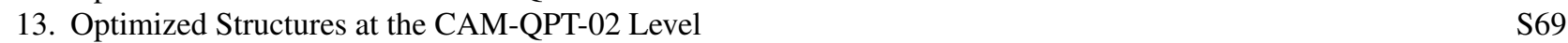

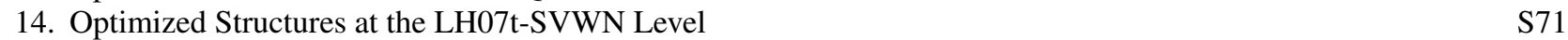

\begin{tabular}{|lr} 
15. Optimized Structures at the LH12ct-SsirPW92 Level & S73
\end{tabular}

$\begin{array}{ll}\text { 16. Optimized Structures at the LH14t-calPBE Level } & \text { S75 }\end{array}$

$\begin{array}{ll}\text { 17. Optimized Structures at the LH20t Level } & \text { S77 }\end{array}$

\begin{tabular}{ll}
\hline 18. Optimized Structures at the mPSTS Level & S79
\end{tabular}

\begin{tabular}{|l|l}
\hline 19. Optimized Structures at the LHJ14 Level & S81
\end{tabular}

S5. NMR Shielding Data 28

\begin{tabular}{|l|l} 
A. NMR Shieldings and Shifts of the ${ }^{1} \mathrm{H}$ and ${ }^{13} \mathrm{C}$ Test Set & S83
\end{tabular}

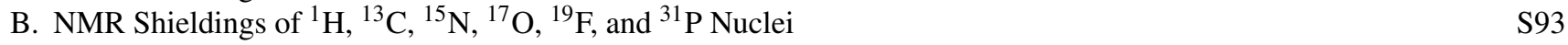

\begin{tabular}{lr}
\hline C. NMR Shieldings and Shifts of Transition-Metal Oxo Compounds & S99
\end{tabular}

\begin{tabular}{lr}
\hline 1. NMR Data & S99 \\
\hline 2. Optimized Struturesat he HFLevel & S100
\end{tabular}

\begin{tabular}{|lr}
\hline 2. Optimized Structures at the HF Level & S100
\end{tabular}

\begin{tabular}{ll}
\hline 3. Optimized Structures at the KT3 Level & S103
\end{tabular}

\begin{tabular}{|lr}
\hline 4. Optimized Structures at the BP86 Level] S105 & S105
\end{tabular}

\begin{tabular}{|ll}
\hline 5. Optimized Structures at the PBE Level & S107
\end{tabular}

$\begin{array}{ll}\text { 6. Optimized Structures at the TPSS Level } & \text { S109 }\end{array}$

\begin{tabular}{ll}
\hline 7. Optimized Structures at the BH\&HLYP Level & S111
\end{tabular}

$\begin{array}{ll}\text { 8. Optimized Structures at the B3LYP Level } & \text { S113 }\end{array}$

$\begin{array}{lr}\text { 9. Optimized Structures at the PBE0 Level } & \text { S115 }\end{array}$

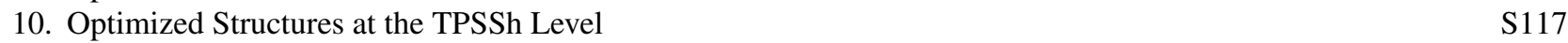

\begin{tabular}{lr}
\hline 11. Optimized Structures at the LC- $\omega$ PBE Level & S119
\end{tabular}

$\begin{array}{lr}\text { 12. Optimized Structures at the } \omega \text { B97X-D Level } & \text { S121 }\end{array}$

\begin{tabular}{|l|l}
\hline 13. Optimized Structures at the CAM-B3LYP Level & S123
\end{tabular}

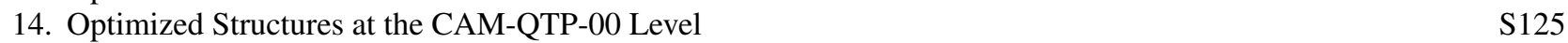

\begin{tabular}{|l|l}
\hline 15. Optimized Structures at the CAM-QTP-02 Level & S127
\end{tabular}

\begin{tabular}{|rr}
\hline 16. Optimized Structures at the LH07t-SVWN Level & S129
\end{tabular}

\begin{tabular}{ll}
\hline 17. Optimized Structures at the LH12ct-SsirPW92 Level & S131
\end{tabular} 
18. Optimized Structures at the LH14t-calPBE Level $\quad$ S133

19. Optimized Structures at the LH20t Level $\quad$ S135

\begin{tabular}{lr}
\hline 20. Optimized Structures at the mPSTS Level & S137
\end{tabular}

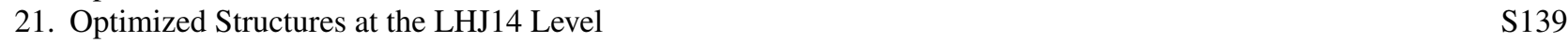

\begin{tabular}{|rr}
\hline D. NMR Shieldings and Shifts of Xenon Fluorides & S141
\end{tabular}

\begin{tabular}{|ll}
\hline 1. NMR Data & S141 \\
\hline
\end{tabular}

\begin{tabular}{ll}
\hline 2. Optimized Structures at the HF Level & S142
\end{tabular}

$\begin{array}{ll}\text { 3. Optimized Structures at the KT3 Level } & \text { S142 }\end{array}$

$\begin{array}{ll}\text { 4. Optimized Structures at the BP86 Level } & \text { S143 }\end{array}$

\begin{tabular}{|lc} 
5. Optimized Structures at the PBE Level & S143
\end{tabular}

$\begin{array}{lr}\text { 6. Optimized Structures at the TPSS Level } & \text { S144 }\end{array}$

\begin{tabular}{ll}
\hline 7. Optimized Structures at the BH\&HLYP Level & S144
\end{tabular}

\begin{tabular}{ll}
\hline 8. Optimized Structures at the B3LYP Level & S145
\end{tabular}

\begin{tabular}{ll}
\hline 9. Optimized Structures at the PBE0 Level & S145
\end{tabular}

\begin{tabular}{|cc}
\hline 10. Optimized Structures at the TPSSh Level & S146
\end{tabular}

\begin{tabular}{|l|l}
\hline 11. Optimized Structures at the LC- $\omega$ PBE Level & S146
\end{tabular}

$\begin{array}{lr}\text { 12. Optimized Structures at the } \omega \text { B97X-D Level } & \text { S147 }\end{array}$

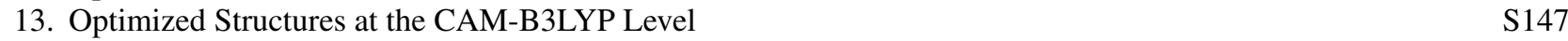

\begin{tabular}{|l|l}
\hline 14. Optimized Structures at the CAM-QTP-00 Level & S148
\end{tabular}

\begin{tabular}{|l|l}
\hline 15. Optimized Structures at the CAM-QTP-02 Level & S148
\end{tabular}

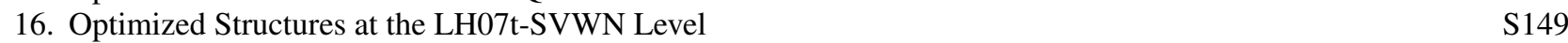

$\begin{array}{ll}\text { 17. Optimized Structures at the LH12ct-SsirPW92 Level } & \text { S149 }\end{array}$

$\begin{array}{lr}\text { 18. Optimized Structures at the LH14t-calPBE Level } & \text { S150 }\end{array}$

\begin{tabular}{|l|l}
\hline 19. Optimized Structures at the LH20t Level & S150
\end{tabular}

\begin{tabular}{|ll}
\hline 20. Optimized Structures at the mPSTS Level & S151
\end{tabular}

\begin{tabular}{|l|l}
\hline 21. & Optimized Structures at the LHJ14 Level
\end{tabular}

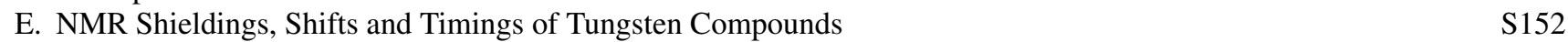

\begin{tabular}{|lr} 
1. NMR Shieldings and Shifts & S152
\end{tabular}

$\begin{array}{ll}\text { 2. Timings } & \text { S157 }\end{array}$

\begin{tabular}{ll}
\hline 3. Optimized Structures at the HF Level & S160
\end{tabular}

$\begin{array}{ll}\text { 4. Optimized Structures at the KT3 Level } & \text { S162 }\end{array}$

$\begin{array}{ll}\text { 5. Optimized Structures at the BP86 Level } & \text { S164 }\end{array}$

$\begin{array}{ll}\text { 6. Optimized Structures at the PBE Level } & \text { S166 }\end{array}$

\begin{tabular}{ll}
\hline 7. Optimized Structures at the TPSS Level & S168
\end{tabular}

\begin{tabular}{lr}
\hline 8. Optimized Structures at the BH\&HLYP Level & S170
\end{tabular}

9. Optimized Structures at the B3LYP Level $\quad S 172$

\begin{tabular}{lr}
\hline 10. Optimized Structures at the PBE0 Level & S174
\end{tabular}

\begin{tabular}{|l|l} 
11. Optimized Structures at the TPSSh Level & S176
\end{tabular}

\begin{tabular}{lr}
\hline 12. Optimized Structures at the LC- $\omega$ PBE Level & S178
\end{tabular}

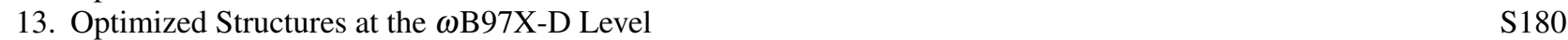

\begin{tabular}{ll}
\hline 14. Optimized Structures at the CAM-B3LYP Level & S182
\end{tabular}

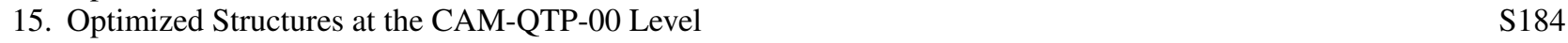

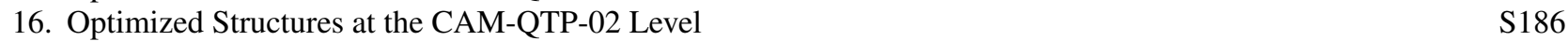

\begin{tabular}{|l|l} 
17. Optimized Structures at the LH07t-SVWN Level & S188
\end{tabular}

$\begin{array}{lr}\text { 18. Optimized Structures at the LH12ct-SsirPW92 Level } & \text { S190 }\end{array}$

19. Optimized Structures at the LH14t-calPBE Level $\quad S 192$

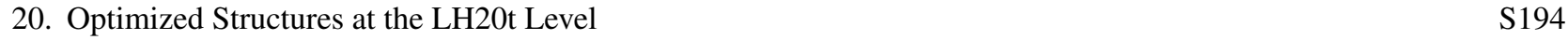

\begin{tabular}{|l|l}
\hline 21. Optimized Structures at the mPSTS Level & S196
\end{tabular}

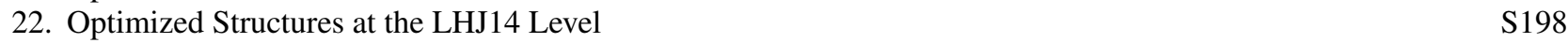

\begin{tabular}{lr}
\hline S6. Magnetically Induced Current Densities & S200
\end{tabular}

$\begin{array}{lr}\text { References } & \text { S203 }\end{array}$ 


\section{S1. TD-DFT DATA}

\section{A. Excitation Energies for the Small Organic Molecule Test Set}

The following subsection lists the 41 adiabtic singlet excitation energies obtained from the test set of Jacquemin and coworkers of Ref. 1. We adopt the same strategy as the authors of Ref. 1, making their results strictly comparable to those reported within this work. Based on the provided structures, the excitation energies are calculated and corrected by the reported B3LYP zero-point vibrational energies [2] (ZPVEs) to yield the 0-0 excitation energy. All calculations use the aug-cc-pVTZ basis set [3-5]. Tight thresholds were applied for the self-consistent field (SCF) procedure; energies were converged up to $10^{-9} \mathrm{E}_{\mathrm{h}}$ and a threshold of $10^{-7}$ a.u. was applied for the root mean square of the density matrix. A threshold of $10^{-5}$ a.u. was used for the norm of the residuum to indicate the convergence of the response equations for time-dependent density functional theory (TD-DFT) [6]. Large grids (grid size 4) were employed for the numerical integration of the DFT parts [7]. The kinetic energy density, $\tau$, is generalized using the paramagnetic current density [8,9]. All calculations in this work were carried out with TURBOMOLE [10.14].

TABLE S1. Mean signed error, mean averaged error, standard deviation, and maximum error of the adiabatic excitation energies of 41 singlet excitation energies from the test set described in Ref. 1] All calculations carried out using listed functional and the aug-cc-pVTZ basis set. Ground-state and excited state geometries taken from Ref. 1. All values are in eV.

\begin{tabular}{lrrrr}
\hline & MSE & MAE & STD & Max. Err. \\
\hline BP86 & -0.198 & 0.237 & 0.227 & 0.657 \\
PBE & -0.204 & 0.240 & 0.224 & 0.632 \\
TPSS & -0.114 & 0.204 & 0.238 & 0.643 \\
BH\&HLYP & -0.005 & 0.387 & 0.450 & 0.757 \\
B3LYP & -0.192 & 0.265 & 0.292 & 0.828 \\
PBE0 & -0.138 & 0.273 & 0.297 & 0.734 \\
TPSSh & -0.039 & 0.211 & 0.249 & 0.570 \\
LC- $\omega$ PBE & -0.021 & 0.291 & 0.328 & 0.588 \\
$\omega B 97 X-D$ & -0.097 & 0.273 & 0.313 & 0.693 \\
CAM-B3LYP & -0.100 & 0.274 & 0.317 & 0.709 \\
CAM-QTP-00 & 0.200 & 0.477 & 0.519 & 1.061 \\
CAM-QTP-02 & 0.128 & 0.363 & 0.404 & 0.790 \\
LH07t & 0.027 & 0.221 & 0.259 & 0.509 \\
LH12ct & 0.015 & 0.754 & 1.014 & 2.863 \\
LH14t & 0.019 & 0.225 & 0.264 & 0.494 \\
LH20t & 0.028 & 0.225 & 0.264 & 0.502 \\
LHJ14 & -0.055 & 0.212 & 0.240 & 0.527 \\
mPSTS & 0.014 & 0.218 & 0.262 & 0.580 \\
\hline
\end{tabular}




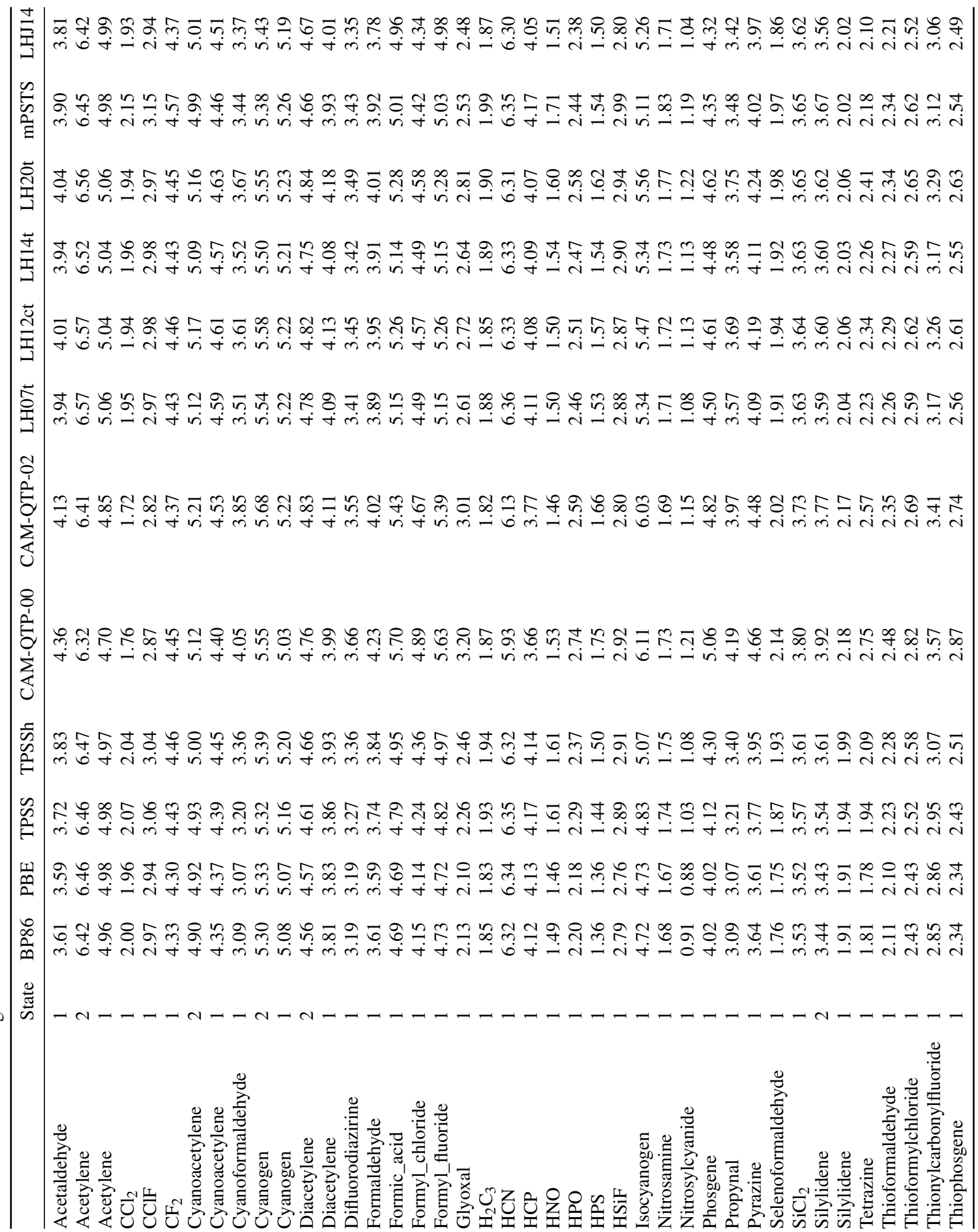




\section{B. Excitation Energies for the Metal-Organic Test Set}

In the second test set, molecules with metal centers are considered. We tested a set of DFAs on a set of molecules, namely: $\mathrm{Ag}_{2}, \mathrm{CdH}, \mathrm{CrF}_{6}, \mathrm{CuCl}, \mathrm{MnO}_{4}^{-}, \mathrm{MoF}_{6},\left[\mathrm{Ni}(\mathrm{CO})_{4}\right],\left[\mathrm{Rh}(\mathrm{bpy})_{3}\right]^{3+}$ (bpy = 2,2-bipyridine), $\left[\mathrm{Ru}(\mathrm{bpy})_{3}\right]^{2+}, \mathrm{TcO}_{4}^{-}, \mathrm{YO}, \mathrm{ZnH}$, and $[\mathrm{Zn}($ porph $)]$ (porph = porphyrin). All structures were optimized using the B3LYP/def2-TZVPP method [15]. For the molecules $\mathrm{CrF}_{6}$ (in matrix)[16], $\mathrm{MoF}_{6}$ (in gas phase)[17], $\mathrm{MnO}_{4}$ (in gas phase)[18], [Ni(CO) $)_{4}$ (in matrix)[19] , [Rh(bpy) $]^{3+}$ (in $\mathrm{MeOH}$ )[20], [ $\left.\mathrm{Ru}(\mathrm{bpy})_{3}\right]^{2+}$ (in vaccum)[21], $\mathrm{TcO}_{4}^{-}$(in gas phase)[22], and [ $\mathrm{Zn}($ porph)] (in $\mathrm{MeOH}$ )[23] the experimental values of the maxima of the peak positions are used. Excited-state TD-DFT calculations were then performed at the optimized geometry again using the def2-TZVPP basis set. For comparison with experiment in this test set, the vertical excitation energies from TD-DFT are directly compared to the experimentally obtained maximum peak position. While this is not fully correct, as vertical excitation energies lack the effects of geometry relaxation and ZPVE, this is a common procedure used in studies for extended molecular systems. Tight thresholds were applied for the self-consistent field procedure; energies were converged up to $10^{-9} \mathrm{E}_{\mathrm{h}}$ and a threshold of $10^{-7}$ a.u. was applied for the root mean square of the density matrix. Geometries were considered converged when the energy change between optimization steps falls below $10^{-6} \mathrm{E}_{\mathrm{h}}$. A threshold of $10^{-5} \mathrm{a}$.u. was used for the norm of the residuum to indicate the convergence of the response equations. Large grids (grid size 4) were employed for the numerical integration of the DFT parts [7]. The kinetic energy density, $\tau$, is generalized using the paramagnetic current density [8, 9].

TABLE S3. Mean signed error, mean averaged error, standard deviation, and maximum error of the vertical excitation energies of 26 singlet excitation energies from the test set described in the main manuscript. All calculations carried out using listed functional and the def2-TZVPP basis set. All values are in $\mathrm{eV}$.

\begin{tabular}{lcccc}
\hline & MSE & MAE & STD & Max. Err. \\
\hline BP86 & 0.371 & -0.108 & 0.434 & 0.841 \\
PBE & 0.363 & -0.126 & 0.432 & 0.848 \\
TPSS & 0.336 & -0.089 & 0.414 & 0.874 \\
BH\&HLYP & 0.371 & 0.366 & 0.423 & 1.048 \\
B3LYP & 0.255 & 0.160 & 0.314 & 0.864 \\
PBE0 & 0.238 & 0.200 & 0.291 & 0.818 \\
TPSSh & 0.358 & 0.463 & 0.446 & 1.573 \\
LC- $\omega$ PBE & 1.146 & 4.099 & 1.490 & 7.902 \\
$\omega B 97 X-D$ & 0.222 & 0.076 & 0.304 & 0.857 \\
CAM-B3LYP & 0.272 & 0.341 & 0.329 & 0.988 \\
CAM-QTP-00 & 0.215 & 0.134 & 0.303 & 0.920 \\
CAM-QTP-02 & 0.268 & 0.027 & 0.354 & 0.901 \\
LH07t & 0.000 & 0.000 & 0.000 & 0.874 \\
LH12ct & 0.227 & 0.156 & 0.295 & 0.886 \\
LH14t & 0.223 & 0.107 & 0.298 & 0.855 \\
LH20t & 0.213 & 0.163 & 0.275 & 0.832 \\
LHJ14 & 0.274 & 0.053 & 0.361 & 0.919 \\
mPSTS & 0.247 & 0.055 & 0.323 & 0.792 \\
\hline
\end{tabular}




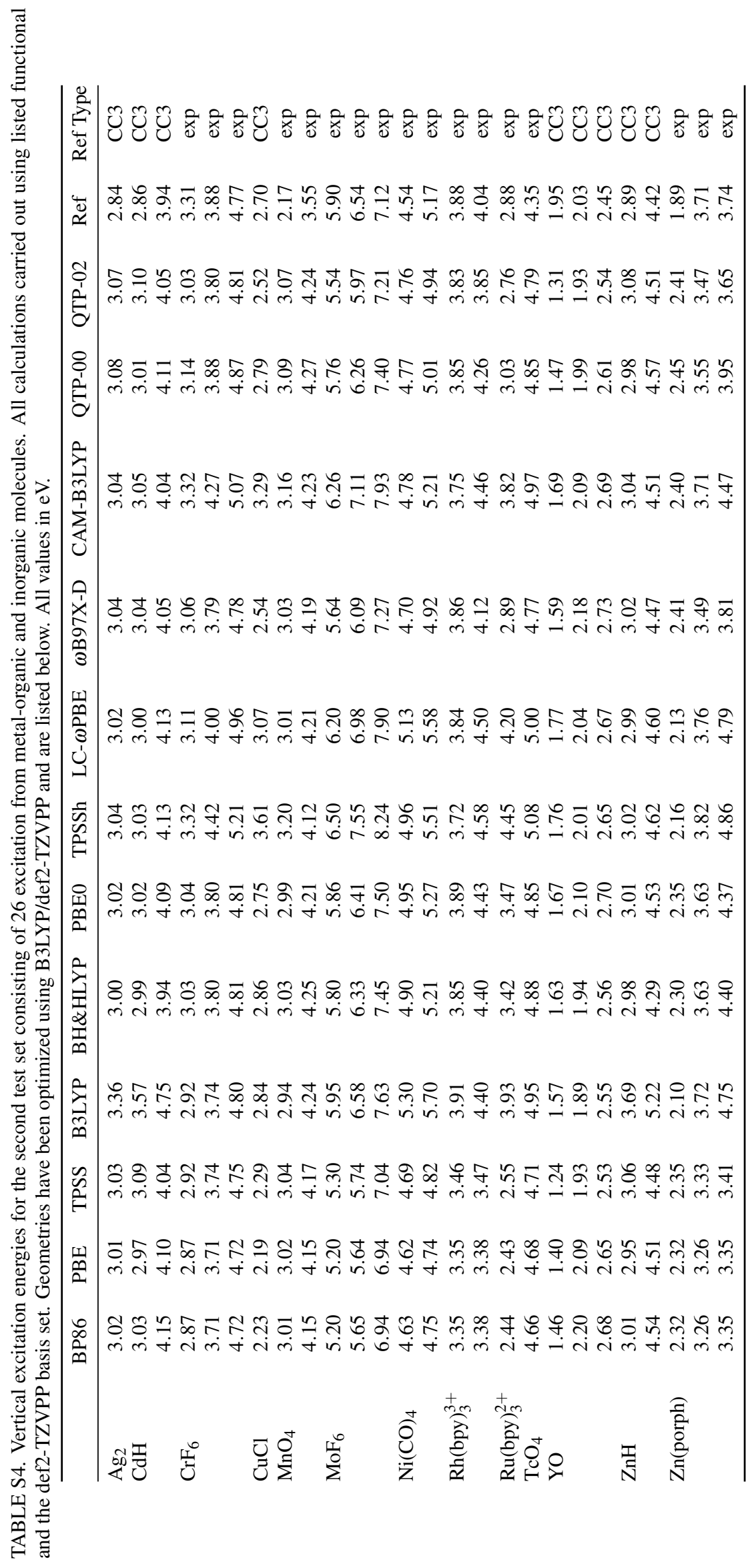




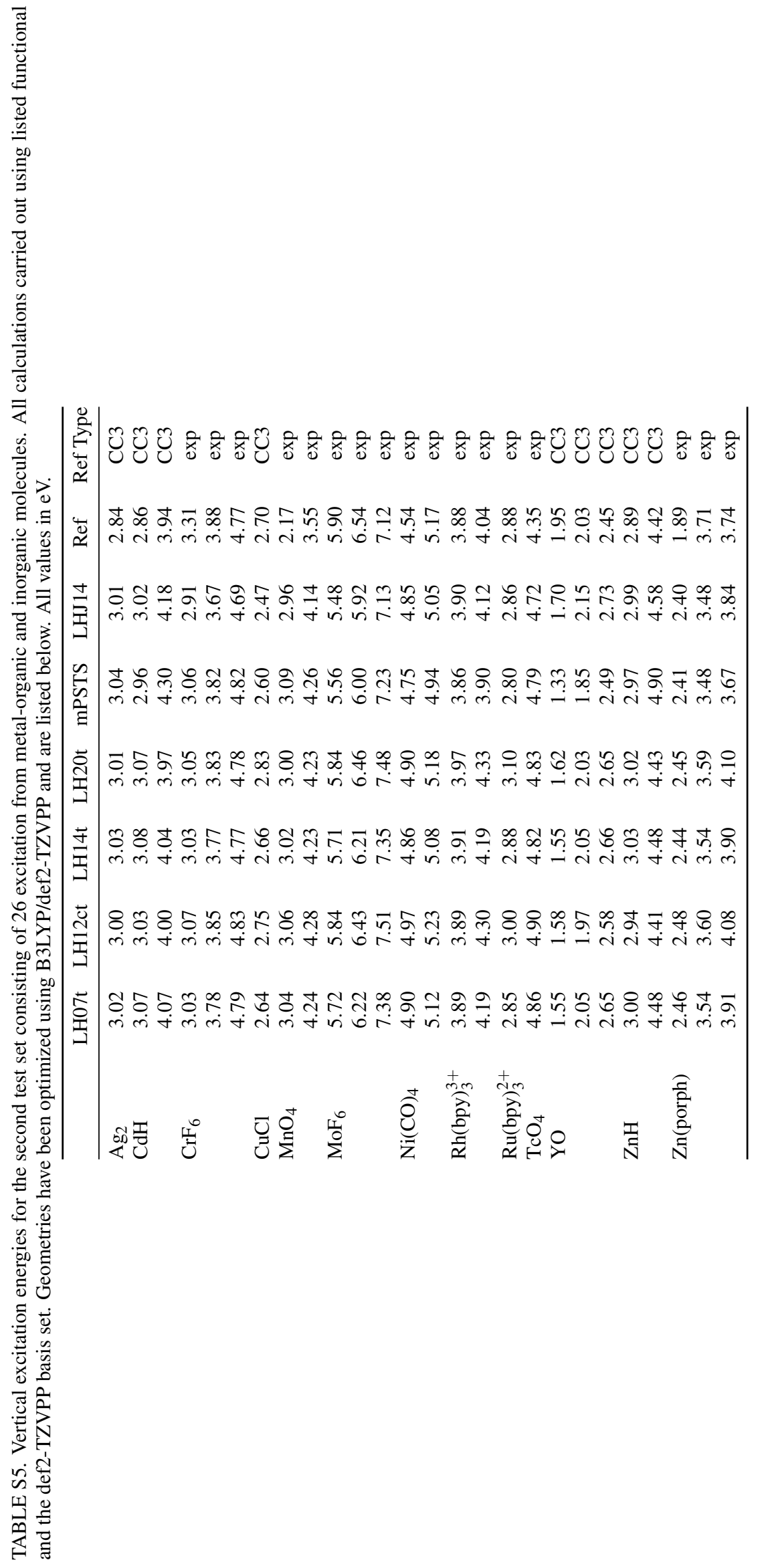




\section{Optimized Structures at the B3LYP/def2-TZVPP level}

The Cartesian coordinates of the structures are listed in Ångström.

$\begin{array}{lrrr}\mathrm{Ag} & 0.0000000 & 0.0000000 & -1.2955745 \\ \mathrm{Ag} & 0.0000000 & 0.0000000 & 1.2955745 \\ & & & \\ \mathrm{Cd} & 0.0000000 & 0.0000000 & -0.8925456 \\ \mathrm{H} & -0.0000000 & 0.0000000 & 0.8925456 \\ & & & \\ & & & \\ \mathrm{Cr} & -0.0000000 & -0.0000000 & -0.0000000 \\ \mathrm{~F} & 1.7265081 & -0.0000000 & 0.0000000 \\ \mathrm{~F} & 0.0000000 & -0.0000000 & -1.7265081 \\ \mathrm{~F} & 0.0000000 & 1.7265081 & -0.0000000 \\ \mathrm{~F} & 0.0000000 & 0.0000000 & 1.7265081 \\ \mathrm{~F} & -0.0000000 & -1.7265081 & -0.0000000 \\ \mathrm{~F} & -1.7265081 & 0.0000000 & -0.0000000\end{array}$

$\begin{array}{lrrr}\mathrm{Cu} & 0.0000000 & 0.0000000 & -1.0459975 \\ \mathrm{Cl} & 0.0000000 & -0.0000000 & 1.0459975\end{array}$

$\begin{array}{llll}\text { Mn } & -0.0000000 & 0.0000000 & 0.0000000\end{array}$

$\begin{array}{llll}0 & 0.9236560 & 0.9236560 & -0.9236560\end{array}$

$\begin{array}{llll}0 & -0.9236560 & -0.9236560 & -0.9236560\end{array}$

$\begin{array}{llll}0 & 0.9236560 & -0.9236560 & 0.9236560\end{array}$

$\begin{array}{lllll}0 & -0.9236560 & 0.9236560 & 0.9236560\end{array}$

$\begin{array}{llll}\text { Mo } & 0.0000000 & 0.0000000 & -0.0000000\end{array}$

$\begin{array}{llll}\text { F } & 0.0000000 & 1.8295396 & 0.0000000\end{array}$

F $\quad 0.0000000 \quad 0.0000000 \quad 1.8295396$

$\begin{array}{llll}\text { F } & 1.8295396 & -0.0000000 & 0.0000000\end{array}$

$\begin{array}{llll}\text { F } & 0.0000000 & 0.0000000 & -1.8295396\end{array}$

$\begin{array}{llll}F & -1.8295396 & 0.0000000 & 0.0000000\end{array}$

$\begin{array}{llll}\text { F } & -0.0000000 & -1.8295396 & 0.0000000\end{array}$

$\begin{array}{lrrr}\mathrm{Ni} & 0.0000000 & -0.0000000 & -0.0000000 \\ \mathrm{C} & -1.0652146 & 1.0652146 & 1.0652146 \\ \mathrm{O} & -1.7204062 & 1.7204062 & 1.7204062 \\ \mathrm{C} & 1.0652146 & -1.0652146 & 1.0652146 \\ \mathrm{O} & 1.7204062 & -1.7204062 & 1.7204062 \\ \mathrm{C} & -1.0652146 & -1.0652146 & -1.0652146 \\ \mathrm{O} & -1.7204062 & -1.7204062 & -1.7204062 \\ \mathrm{C} & 1.0652146 & 1.0652146 & -1.0652146 \\ \mathrm{O} & 1.7204062 & 1.7204062 & -1.7204062 \\ & & & \\ & & & \\ \mathrm{C} & -1.6741157 & 4.5482017 & -0.0500736 \\ \mathrm{C} & -0.7382028 & 4.1802040 & -1.0072986 \\ \mathrm{C} & -0.2471639 & 2.8850618 & -0.9949666 \\ \mathrm{C} & -1.5570807 & 2.3214556 & 0.8533938\end{array}$




\begin{tabular}{|c|c|c|c|}
\hline 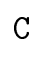 & -2.0837240 & 3.6091542 & 0.8866856 \\
\hline$C$ & -1.9281710 & 1.2580065 & 1.8014497 \\
\hline & -1.6184735 & -0.9735518 & \\
\hline & -2.5056951 & -0.8559589 & 3.470251 \\
\hline$C$ & -3.1192944 & 0.3694077 & $3.6934^{\circ}$ \\
\hline C & -2.8263517 & 1.4322900 & 2.84995 \\
\hline 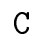 & -2.7076972 & -0.6721073 & -1.26032 \\
\hline $\mathrm{C}$ & -3.5859335 & -0.9448322 & -2.29620 \\
\hline 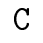 & -3.0964121 & -0.9769952 & -3.59513 \\
\hline $\mathrm{C}$ & -1.7461170 & -0.7384220 & -3.811622 \\
\hline C & -0.9077984 & -0.4711693 & -2.733597 \\
\hline $\mathrm{C}$ & 0.5353703 & -0.2091109 & -2.8610 \\
\hline $\mathrm{C}$ & 1.2225692 & -0.2216942 & -4.071 \\
\hline & 2.5855546 & 0.0409180 & -4.09500 \\
\hline & 3.2394960 & 0.3145516 & -2.901174 \\
\hline & 2.5073247 & 0.3123733 & -1.725382 \\
\hline C & 047 & -3.04 & -0.3 \\
\hline $\mathrm{C}$ & 1.9 & 1.4 & $1 . \varepsilon$ \\
\hline $\mathrm{C}$ & 2.9 & 1.6 & \\
\hline $\mathrm{C}$ & 3.5864682 & 0.47 & \\
\hline & 3.2020740 & -0.7572389 & 2.7 \\
\hline & 2.1685821 & -0.823 & 1.776077 \\
\hline C & $1.6 \mathrm{~s}$ & -2.07 & 1.166158 \\
\hline C & & -3.3266942 & \\
\hline C & & -4.4 & \\
\hline $\mathrm{C}$ & 0.65 & -4.3 & -0 . \\
\hline & -2.0809075 & 5.55 & -0 \\
\hline & -0.389 & $4.87^{\circ}$ & -1.7 \\
\hline & 0.48 & 2.56 & -1.7 \\
\hline & -2.8108919 & 3.8834265 & 1.635552 \\
\hline $\mathrm{H}$ & -1.1 & -1.9 & \\
\hline & -2.7 & -1.7 & \\
\hline H & -3.8 & 0.4 & 4.5 \\
\hline & -3.29 & 2.389 & $3 . c$ \\
\hline & -3.0515164 & -0.6402314 & -0.2 \\
\hline & -4.62 & -1.1 & -2. \\
\hline H & -3.75 & -1.1847587 & -4.429022 \\
\hline $\mathrm{H}$ & -1.3 & $-0.7 €$ & -4.8 \\
\hline $\mathrm{H}$ & & -0.4 & -4.5 \\
\hline $\mathrm{H}$ & 3.1 & 0.0 & -5 \\
\hline $\mathrm{H}$ & 4.2987745 & 0.5 & -2.8 \\
\hline $\mathrm{H}$ & 2.9810663 & 0.5219301 & -0.778828 \\
\hline $\mathrm{H}$ & -0.6712354 & -2.8912893 & -0.9 \\
\hline $\mathrm{H}$ & & 2.3628663 & 1.5 \\
\hline $\mathrm{H}$ & & 2.5945778 & 3.180897 \\
\hline $\mathrm{H}$ & & & \\
\hline $\mathrm{H}$ & & -1.6 & \\
\hline $\mathrm{H}$ & & -3.4264874 & \\
\hline $\mathrm{H}$ & 2.1439277 & -5.4303606 & 1.0 \\
\hline $\mathrm{H}$ & 0.2238639 & -5.1610263 & $-0.57417 \epsilon$ \\
\hline $\mathrm{N}$ & -0.6417395 & 1.9762961 & -0.089993 \\
\hline $\mathrm{N}$ & -1.3347119 & 0.0525720 & 1.596416 \\
\hline $\mathrm{N}$ & -1.4021410 & -0.4421144 & -1.467888 \\
\hline N & 1.1899013 & 0.0591146 & -1.70064 \\
\hline $\mathrm{N}$ & 1.5318874 & 0.3079484 & 1.373080 \\
\hline N & & -1.9501792 & \\
\hline & & & \\
\hline
\end{tabular}




\begin{tabular}{|c|c|c|c|}
\hline $\mathrm{C}$ & -1.7001028 & 4.5723620 & -0.0247146 \\
\hline$C$ & -0.7623750 & 4.2043403 & -0.9806064 \\
\hline & -0.2707309 & 2.9106605 & -0.9677921 \\
\hline & -1.5755126 & 2.3422259 & 0.8690683 \\
\hline$C$ & -2.1065247 & 3.6300283 & 0.906167 \\
\hline$C$ & -1.9490988 & 1.2785557 & 1.815010 \\
\hline C & -1.6490085 & -0.9427715 & 2.42758 \\
\hline $\mathrm{C}$ & -2.5357706 & -0.8240997 & 3.48359 \\
\hline $\mathrm{C}$ & -3.1483518 & 0.4019288 & 3.707336 \\
\hline & -2.8494804 & 1.4590720 & 2.863105 \\
\hline & -2.7039378 & -0.6839621 & -1.302672 \\
\hline & -3.5801670 & -0.9590804 & -2.338105 \\
\hline & -3.0904450 & -0.9911996 & -3.637217 \\
\hline & -1.7426112 & -0.7479537 & -3.846618 \\
\hline & -0.9085106 & -0.4778210 & -2.763607 \\
\hline C & 0.53 & -0.2104262 & -2.891327 \\
\hline $\mathrm{C}$ & & -0.2220907 & \\
\hline $\mathrm{C}$ & $2.5^{\circ}$ & 0.0 & -4 \\
\hline $\mathrm{C}$ & 3.22 & 017 & -2 \\
\hline $\mathrm{C}$ & 2.497 & 2746 & -1.7 \\
\hline & $0.1797^{\circ}$ & 504281 & -0.2 \\
\hline $\mathrm{C}$ & 1.9370163 & 1.4670949 & \\
\hline $\mathrm{C}$ & 2.9 & 1.5888863 & \\
\hline $\mathrm{C}$ & & & \\
\hline $\mathrm{C}$ & 3.2 & -0 . & \\
\hline $\mathrm{C}$ & $2.1 \mathrm{~s}$ & $-0 . \varepsilon$ & 1. \\
\hline $\mathrm{C}$ & 1.71 & -2.0 & 1.1 \\
\hline c & 2.26 & -3.3 & 33 \\
\hline C & 1.74 & -4.4 & \\
\hline $\mathrm{C}$ & 0.68 & -4.3 & $-0 . c$ \\
\hline $\mathrm{H}$ & -2.1 & 5.5 & -0 . \\
\hline $\mathrm{H}$ & -0.4 & 4.9 & -1 \\
\hline $\mathrm{H}$ & 0.45 & 2.5 & -1.6 \\
\hline $\mathrm{H}$ & -2.83 & 3.85 & 1.6 \\
\hline & -1.15 & -1.8808778 & 834 \\
\hline $\mathrm{H}$ & -2.7360541 & -1.6788785 & 4.113428 \\
\hline $\mathrm{H}$ & -3.8451278 & 0.5340149 & 4.523295 \\
\hline $\mathrm{H}$ & -3.314 & 2.4 & 3.022655 \\
\hline $\mathrm{H}$ & -3.0 & -0.6 & -0. \\
\hline $\mathrm{H}$ & -4.6229253 & -1.1 & -2.1 \\
\hline $\mathrm{H}$ & -3.7443344 & -1.2 & -4.472135 \\
\hline $\mathrm{H}$ & -1.3449413 & -0.7675602 & -4.849490 \\
\hline $\mathrm{H}$ & 0.6924173 & -0.4388633 & -5.023938 \\
\hline $\mathrm{H}$ & 3.1129796 & 0.0363131 & -5.074791 \\
\hline $\mathrm{H}$ & 4.2874074 & 0.5339317 & -2.914294 \\
\hline $\mathrm{H}$ & & 0.5 & -0.821211 \\
\hline $\mathrm{H}$ & -0.6475032 & -2.9 & -0.947892 \\
\hline $\mathrm{H}$ & 1.4034578 & 3786 & 1.543733 \\
\hline $\mathrm{H}$ & 3.2280898 & 2.5654292 & 3.19573 \\
\hline $\mathrm{H}$ & 4.4227779 & 0.4966297 & 3.962379 \\
\hline $\mathrm{H}$ & 3.7287660 & -1.6824363 & 3.036474 \\
\hline $\mathrm{H}$ & 3.0907564 & -3.4367580 & 2.14099 \\
\hline $\mathrm{H}$ & 2.1716322 & -5.4470179 & \\
\hline $\mathrm{H}$ & & -5.1785219 & -0.543171 \\
\hline N & -0.6577054 & 1.9900178 & -0.069231 \\
\hline & -1.3534626 & 0.0755477 & 1.602758 \\
\hline & -1.3954142 & -0.4484919 & -1.49509 \\
\hline
\end{tabular}




\begin{tabular}{|c|c|c|c|}
\hline $\mathrm{N}$ & 1.1798233 & 0.0594883 & -1.7269910 \\
\hline $\mathrm{N}$ & 1.5515106 & 0.2869165 & 1.3817897 \\
\hline N & 0.6709765 & -1.9585273 & 0.3200498 \\
\hline $\mathrm{Ru}$ & -0.0010190 & 0.0010908 & 0.0029882 \\
\hline Tc & 0.0000000 & 0.0000000 & -0.0000000 \\
\hline 0 & 0.9897039 & 0.9897039 & -0.9897039 \\
\hline U & -0.9897039 & -0.9897039 & -0.9897039 \\
\hline U & 0.9897039 & -0.9897039 & 0.9897039 \\
\hline 0 & -0.9897039 & 0.9897039 & 0.9897039 \\
\hline & 0.0000000 & 0.0000000 & -0.8990593 \\
\hline 0 & 0.0000000 & 0.0000000 & 0.8990593 \\
\hline $\mathrm{Zn}$ & 0.0000000 & 0.0000000 & -0.812556 \\
\hline & 0.0000000 & 0.0000000 & 0.812556 \\
\hline $\mathrm{Zn}$ & -0.0000000 & 0.0000000 & 0.0000000 \\
\hline C & -2.4222075 & 2.4222075 & 0.0000000 \\
\hline $\mathrm{C}$ & -2.4222075 & -2.4222075 & 0.0000000 \\
\hline $\mathrm{C}$ & 2.4222075 & -2.4222075 & 0.0000000 \\
\hline $\mathrm{C}$ & 2.4222075 & 2.4222075 & 0.000000 \\
\hline $\mathrm{N}$ & 0.0000000 & -2.0526990 & 0.000000 \\
\hline $\mathrm{N}$ & -2.0526990 & 0.0000000 & 0.000000 \\
\hline $\mathrm{N}$ & 0.0000000 & 2.0526990 & 0.0000000 \\
\hline $\mathrm{N}$ & 2.0526990 & -0.0000000 & 0.0000000 \\
\hline $\mathrm{C}$ & 1.1010752 & 2.8633520 & 0.0000000 \\
\hline $\mathrm{C}$ & -1.1010752 & 2.8633520 & 0.0000000 \\
\hline $\mathrm{C}$ & -2.8633520 & 1.1010752 & 0.000000 \\
\hline $\mathrm{C}$ & -2.8633520 & -1.1010752 & 0.000000 \\
\hline $\mathrm{C}$ & 2.8633520 & 1.1010752 & 0.000000 \\
\hline $\mathrm{C}$ & 2.8633520 & -1.1010752 & 0.0000000 \\
\hline $\mathrm{C}$ & 1.1010752 & -2.8633520 & 0.0000000 \\
\hline $\mathrm{C}$ & -1.1010752 & -2.8633520 & 0.0000000 \\
\hline C & 0.6796059 & 4.2 & 0.000000 \\
\hline $\mathrm{C}$ & -0.6796059 & 4.2430079 & 0.000000 \\
\hline $\mathrm{C}$ & -4.2430079 & 0.6796059 & 0.000000 \\
\hline $\mathrm{C}$ & -4.2430079 & -0.6796059 & 0.000000 \\
\hline $\mathrm{C}$ & -0.6796059 & -4.2430079 & 0.000000 \\
\hline $\mathrm{C}$ & 0.6796059 & -4.2430079 & 0.000000 \\
\hline $\mathrm{C}$ & 4.2430079 & 0.6796059 & 0.000000 \\
\hline $\mathrm{C}$ & 4.2430079 & -0.6796059 & 0.000000 \\
\hline $\mathrm{H}$ & 1.3431135 & 5.0932388 & 0.000000 \\
\hline $\mathrm{H}$ & -1.3431135 & 5.0932388 & 0.000000 \\
\hline $\mathrm{H}$ & -5.0932388 & 1.3431135 & 0.000000 \\
\hline $\mathrm{H}$ & -5.0932388 & -1.3431135 & 0.000000 \\
\hline $\mathrm{H}$ & -1.3431135 & -5.0932388 & 0.000000 \\
\hline $\mathrm{H}$ & 1.3431135 & -5.0932388 & 0.000000 \\
\hline $\mathrm{H}$ & 5.0932388 & 1.3431135 & 0.000000 \\
\hline $\mathrm{H}$ & 5.0932388 & -1.3431135 & 0.000000 \\
\hline $\mathrm{H}$ & -3.1874775 & 3.1874775 & 0.000000 \\
\hline $\mathrm{H}$ & -3.1874775 & -3.1874775 & 0.000000 \\
\hline $\mathrm{H}$ & 3.1874775 & -3.1874775 & 0.000000 \\
\hline $\mathrm{H}$ & 3.1874775 & 3.1874775 & 0.000000 \\
\hline
\end{tabular}




\section{S2. $G W$ DATA}

The following subsections list the Kohn-Sham eigenvalues and quasi-particle energies for all molecules in the GW27 [24] and $G W 100$ [25] test sets. Geometries and basis sets were chosen in accordance with the $\operatorname{CCSD}(\mathrm{T})$ reference values provided by Krause and co-workers [26]. All calculations were done using the def2-TZVPP basis set [15, 27], as was used for the reference values. The RI- $J$ approximation was used throughout with suitable auxiliary basis sets taken from the TURBOMOLE basis set library [28-31]. Exchange-correlation terms stemming from the density functional approximations (DFAs) were evaluated using large grids (gridsize 5) [7]. Ground states were converged to at least $10^{-9} \mathrm{E}_{\mathrm{h}}$ in energy with a root mean square of the density below $10^{-7}$. For the subsequent $G_{0} W_{0}$ calculations, "MP2-fitting" type basis sets for the evaluation of the dielectric matrix and related quantities were used [32, 33]. The analytic continuation was performed by a Padé extrapolation using 128 parameters derived from 128 frequencies along the imaginary axis for 15 points between the highest occupied molecular orbital (HOMO) and lowest unoccupied molecular orbital (LUMO) energy levels, shifted by $0.2 \mathrm{eV}$ into the imaginary plane. This shift was reduced to $0.1 \mathrm{eV}$ for calculations on boron nitride employing the BP86 [34, 35] and PBE [36] functionals due to the low HOMO-LUMO gap predicted for these DFAs. All calculations in this work were carried out with TURBOMOLE [10-14, 24, 37,-41]. For comparison ionization potentials using eigenvalue-only self-consistent $G W(\mathrm{ev} G W)[42]$ are provided.

Figure S1. Comparison of various functionals for the calculation of the first ionization potentials for molecules in the GW27 test set using the evGW approximation. LH07t-SVWN, LH12ct-SsirPW92, and LH14t-calPBE are abbreviated with LH07t, LH12ct, and LH14t. MAE: mean-absolute error, Max: maximum absolute error, MSD: mean signed deviation, STD: standard deviation.

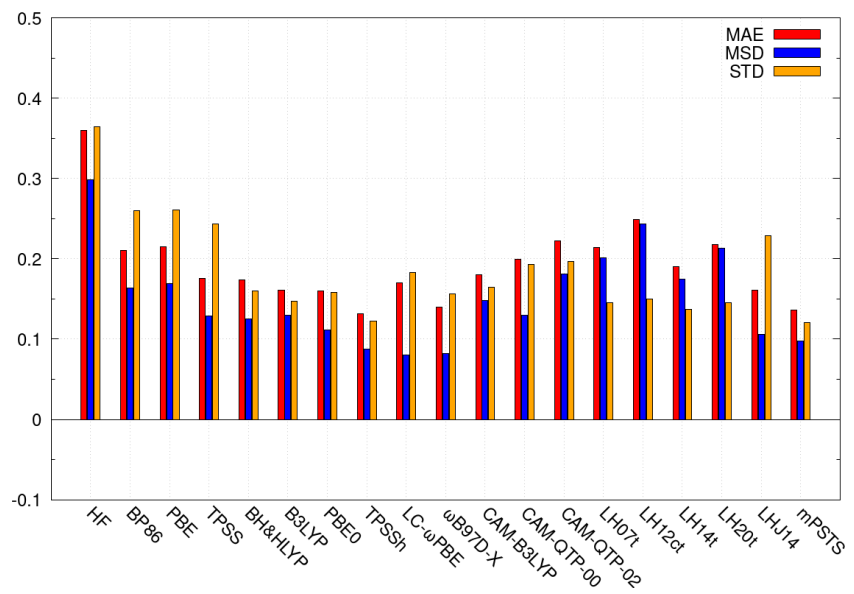

Figure S2. Comparison of various functionals for the calculation of the first ionization potentials for molecules in the $G W 100$ test set using the ev $G W$ approximation. See Figure $\mathrm{S} 1$ for further details regarding abbreviations.

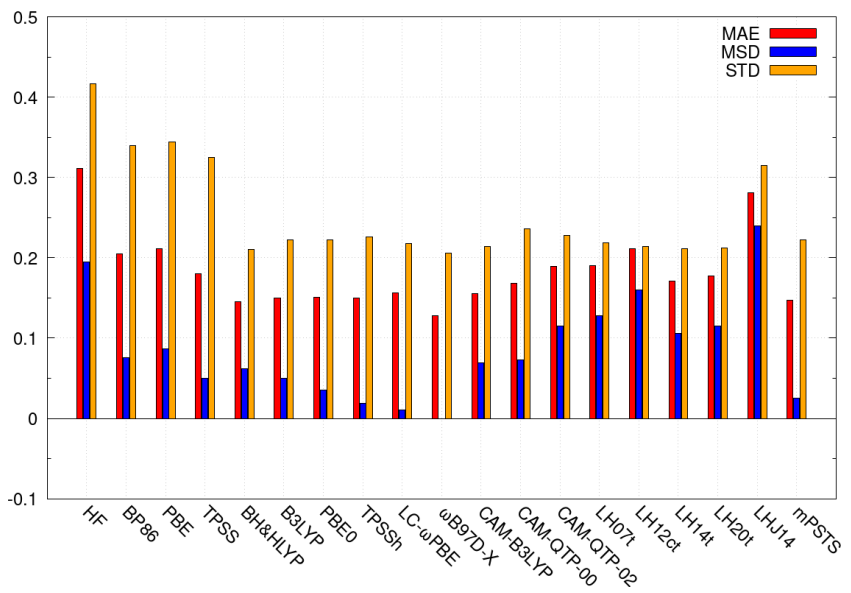




\section{今ั}

苍

这

偣

ए.

$\stackrel{\square}{\square}$

๖ै.

胥

\section{응}

胥

苟

$$
\text { 댈 }
$$$$
\text { 过 }
$$

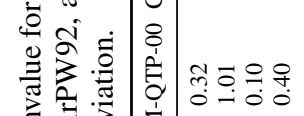

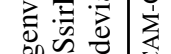

ब

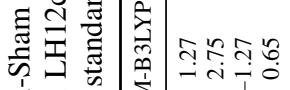

至完

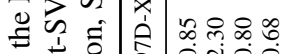

के

可

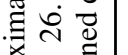

눌 $\frac{500}{5}$

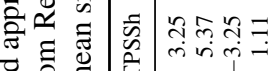

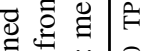

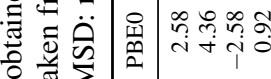

苛言

एँ.

호 $>0$

च

흥 홍

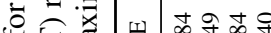

a

$\infty \begin{array}{ccc}\infty & \infty \\ \infty & \infty & \infty\end{array}$

击诜芒

ल चूल

定

ส $:$ व

응

to

శึ 워

吨

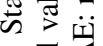

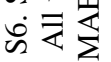

空密守 


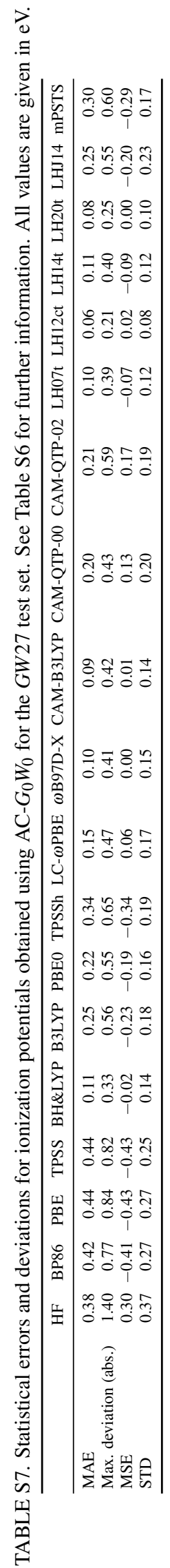




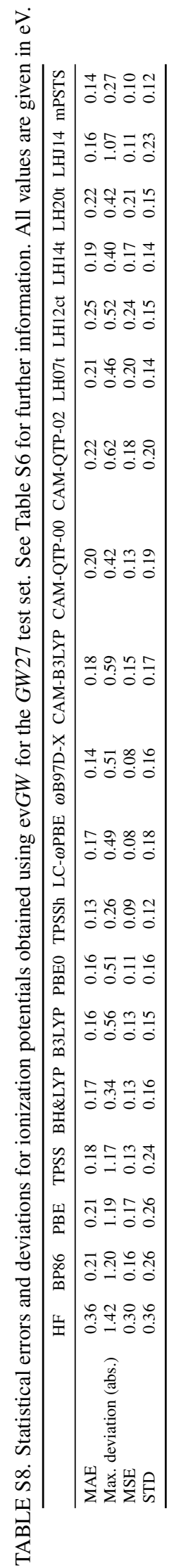




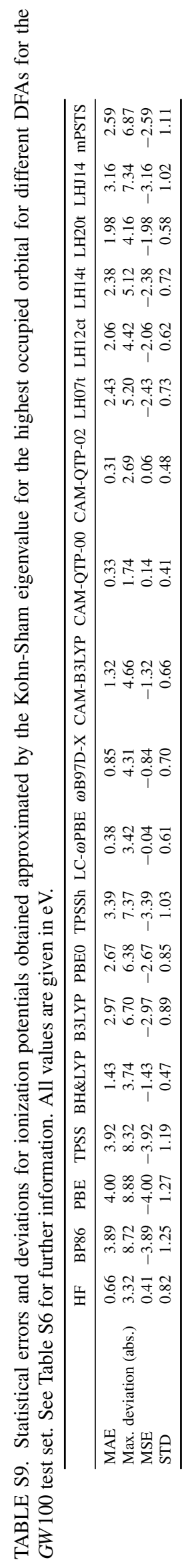




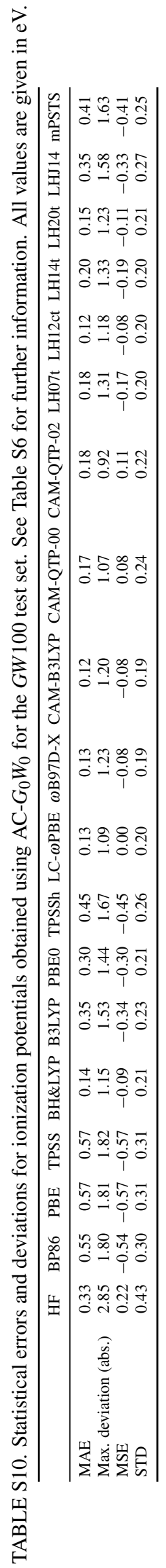




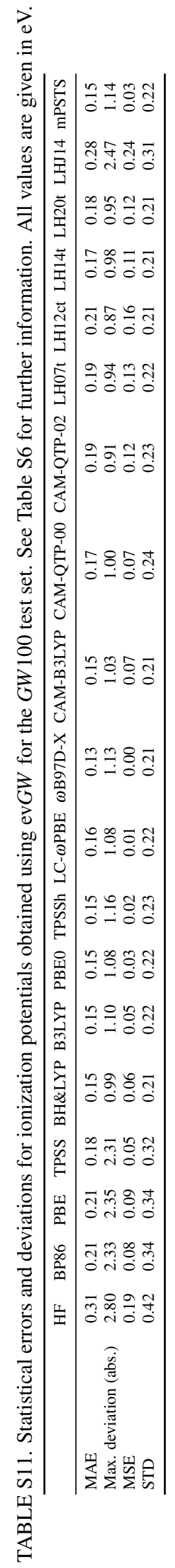


A. Kohn-Sham Eigenvalues for the GW27 and GW100 Test Sets 


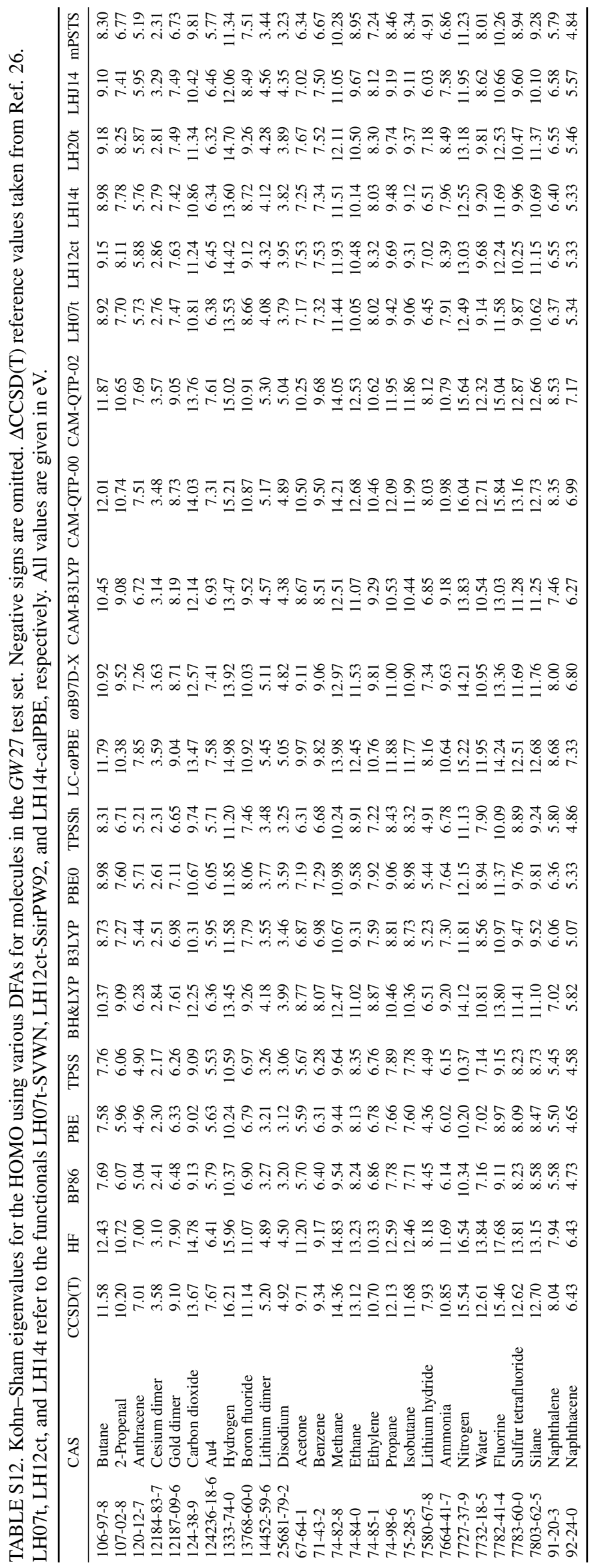




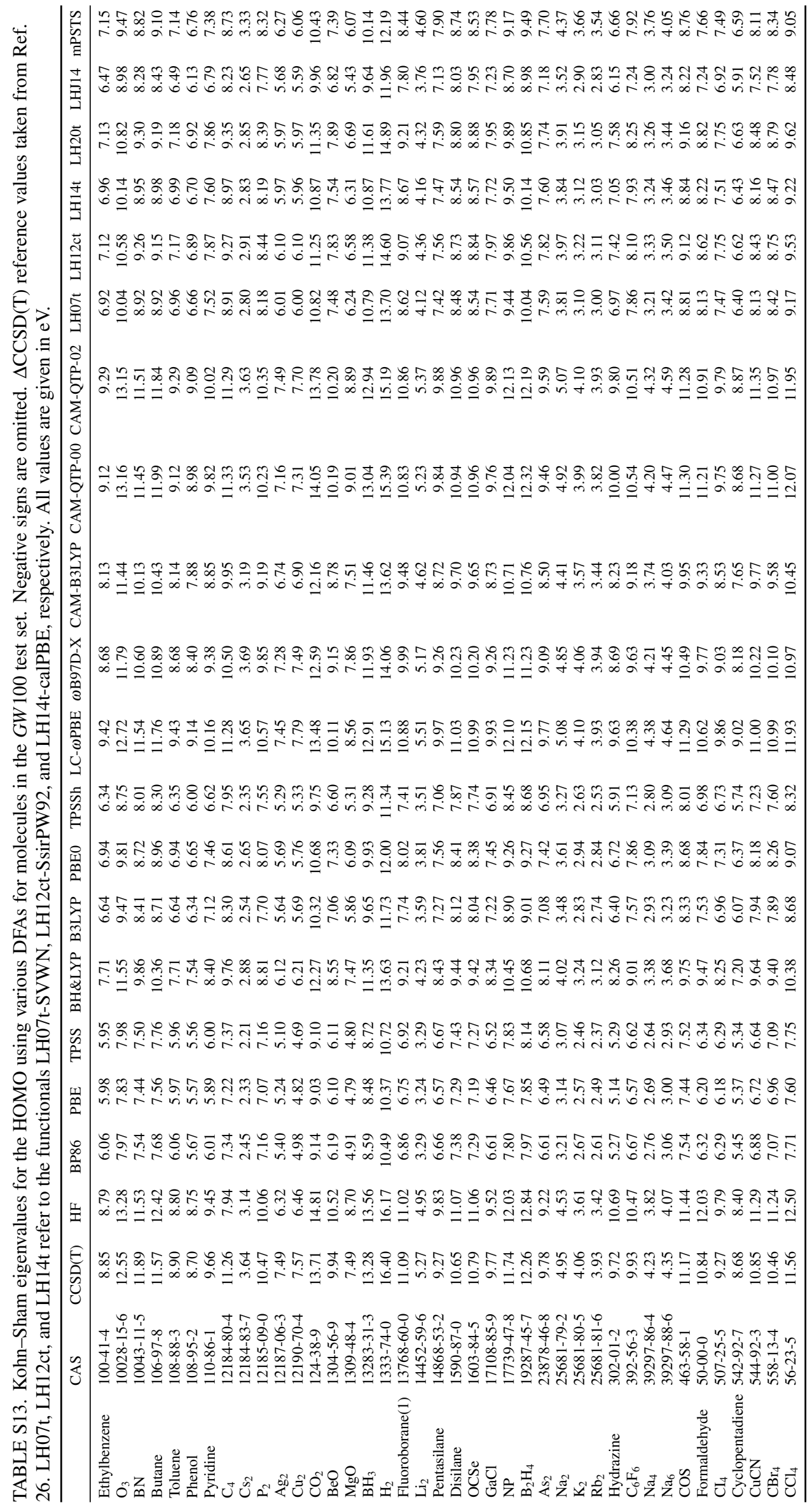




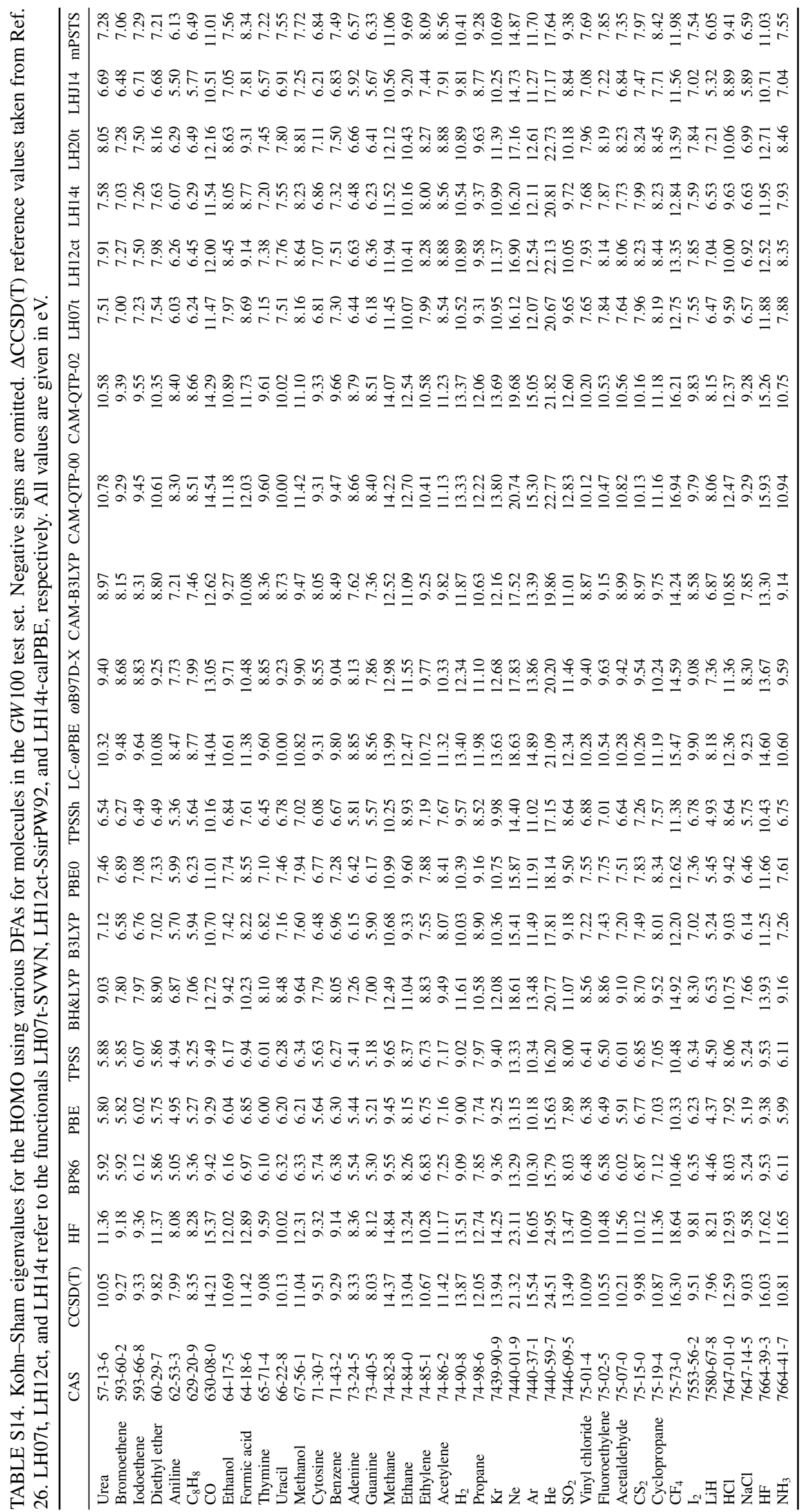




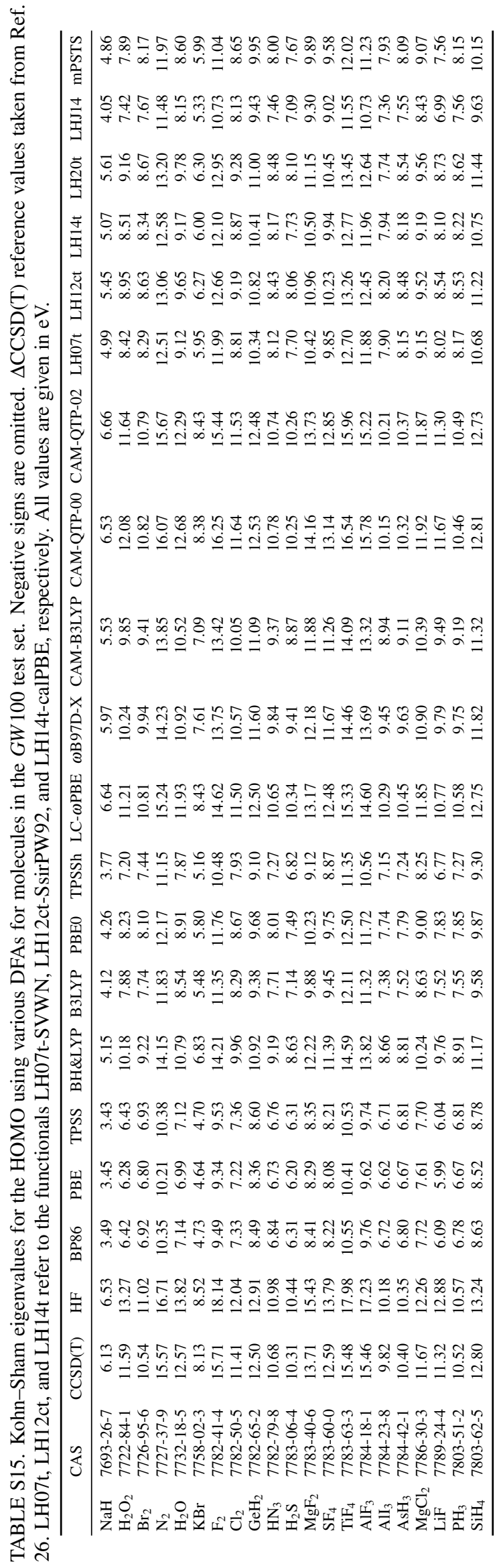


B. Quasi-particle Energies for the GW27 and GW100 Test Sets 


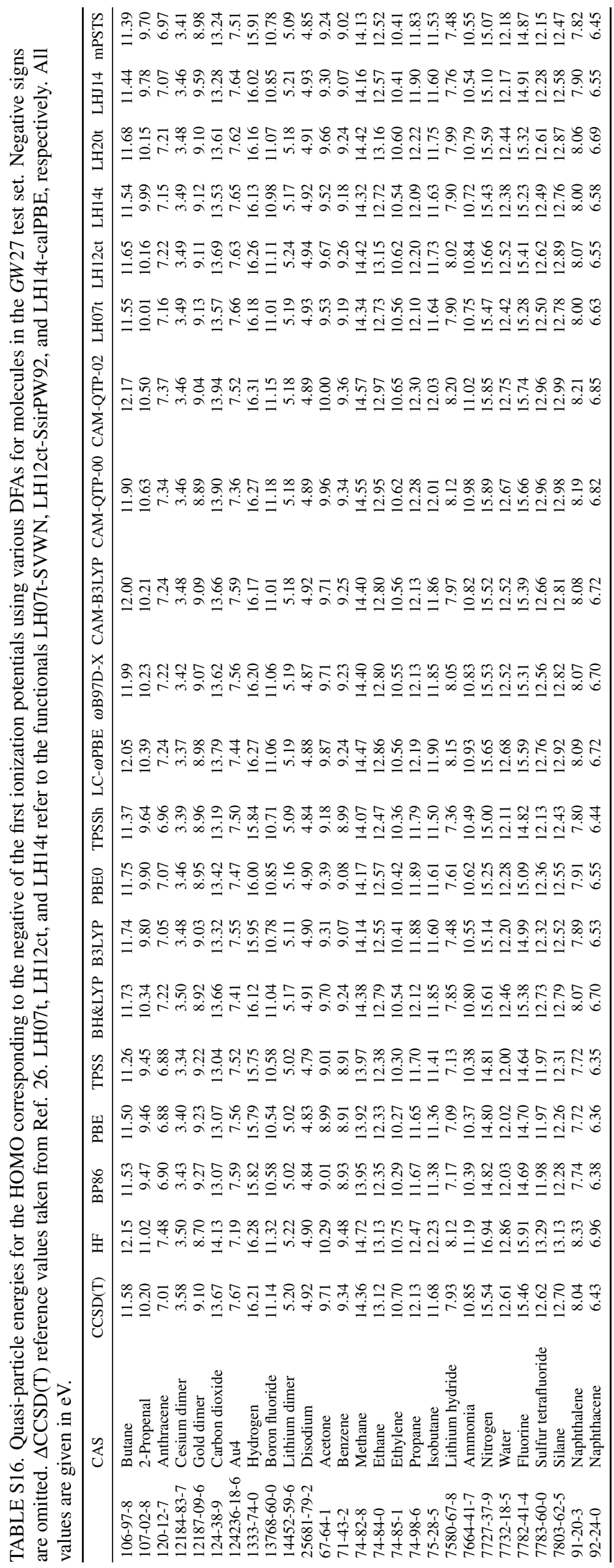




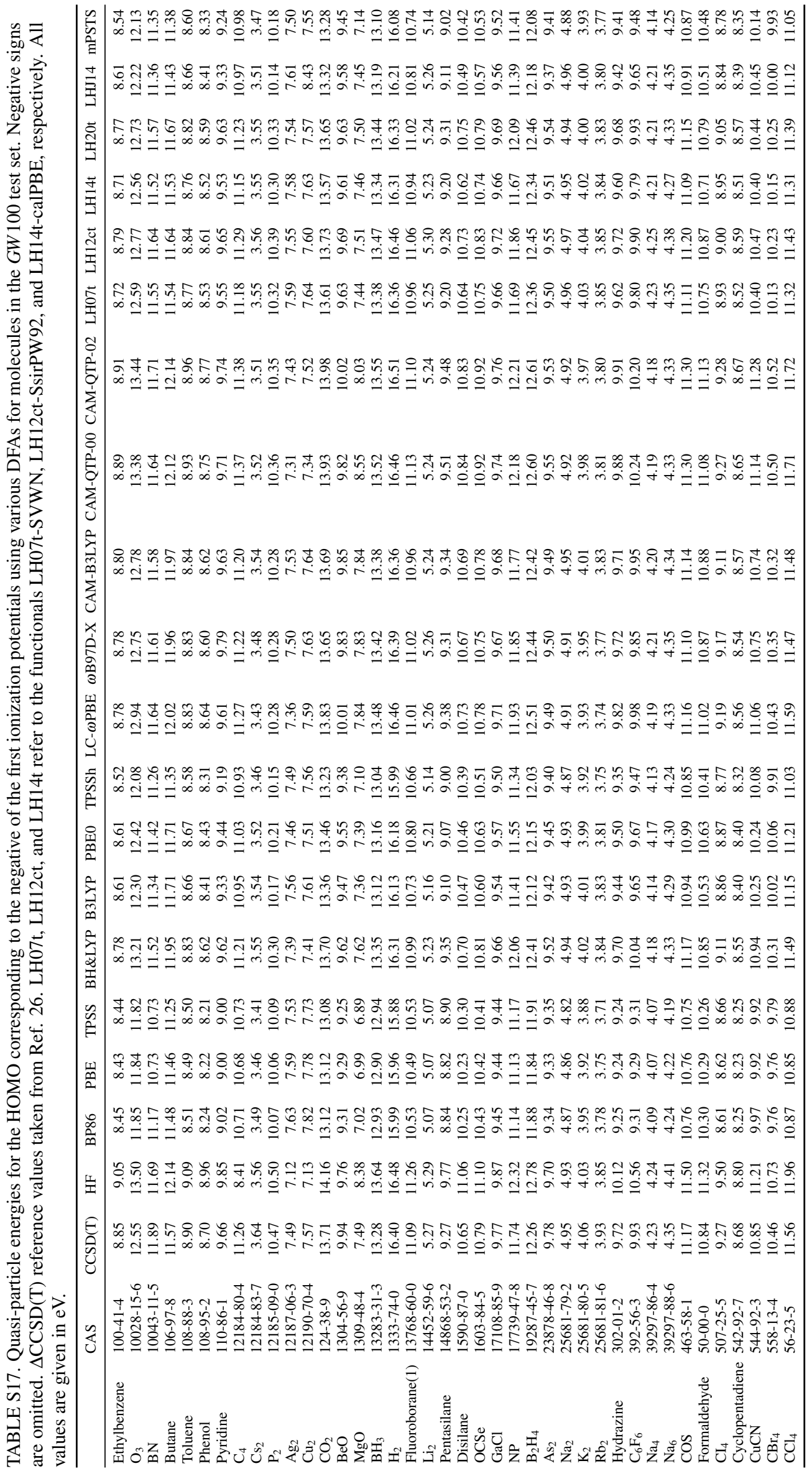




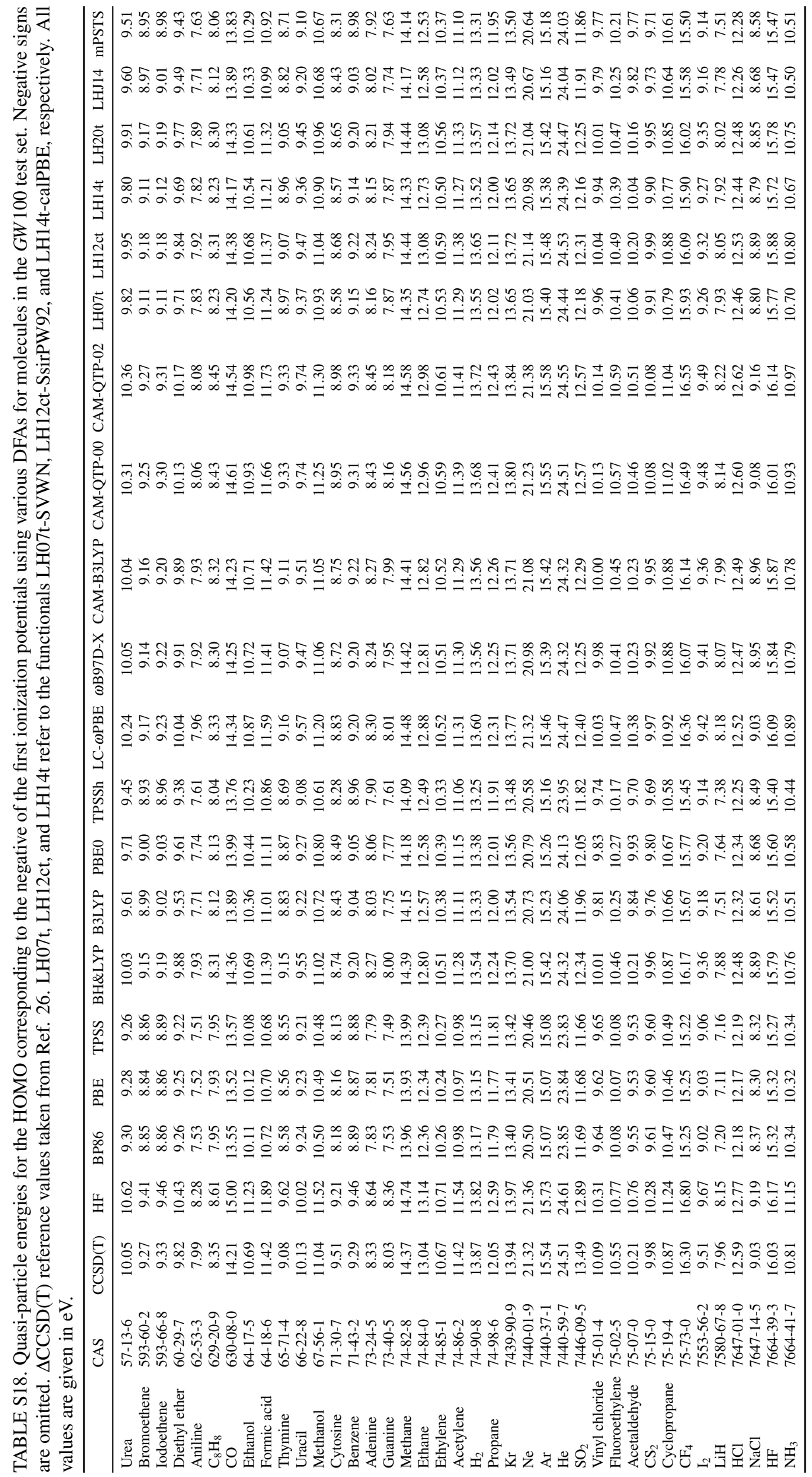




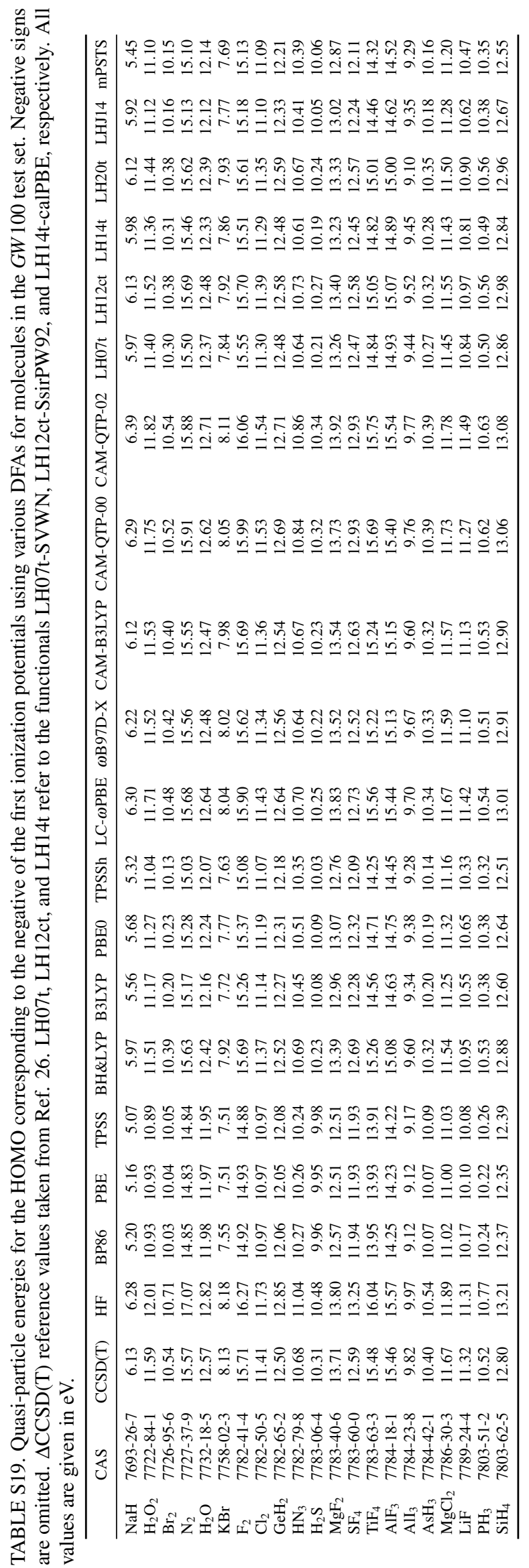


C. Quasi-particle Energies for the GW27 and GW100 Test Sets using evGW 


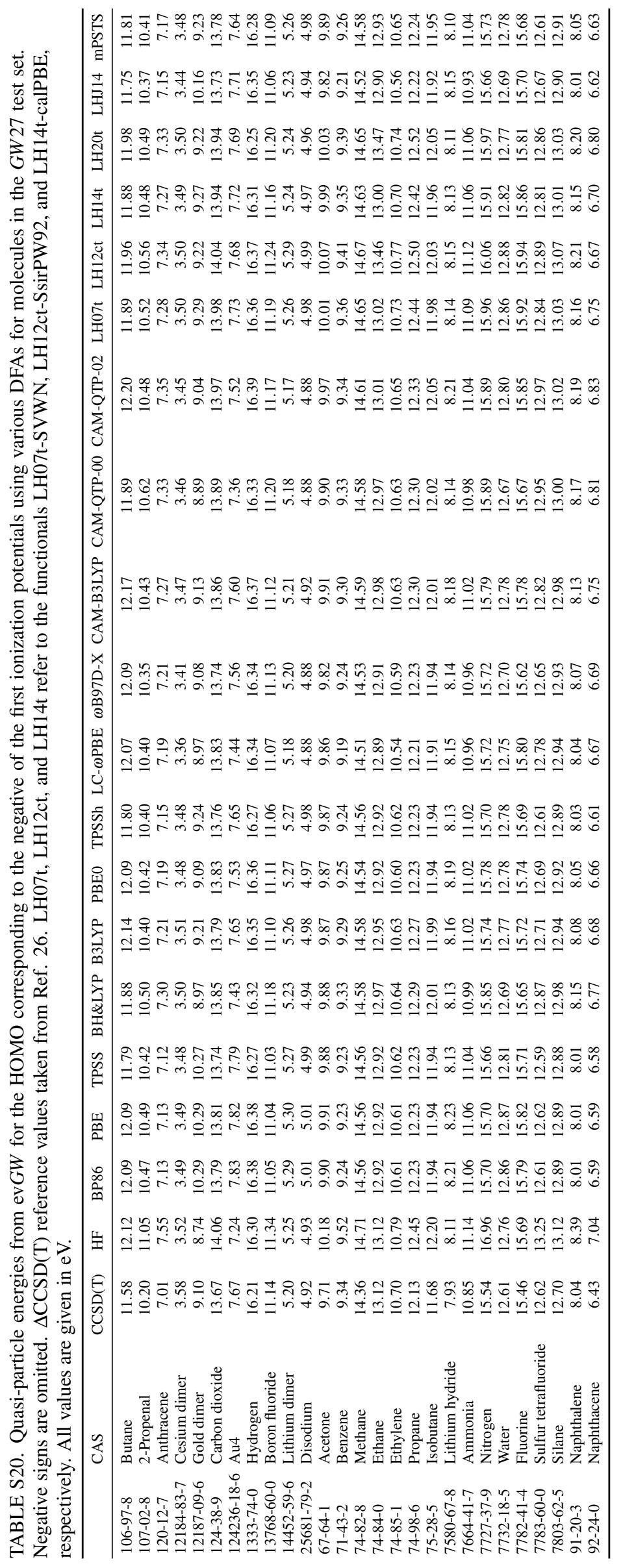




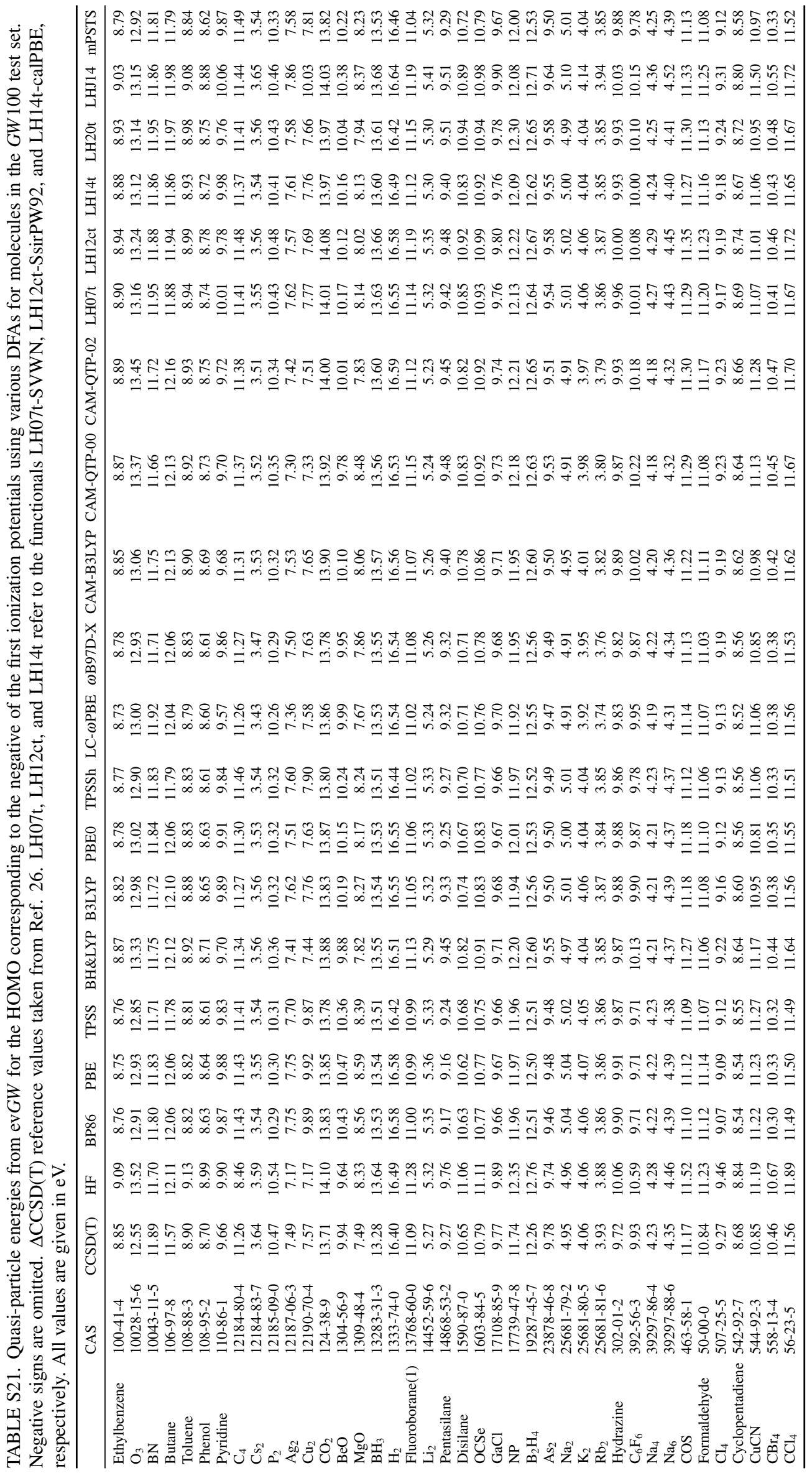




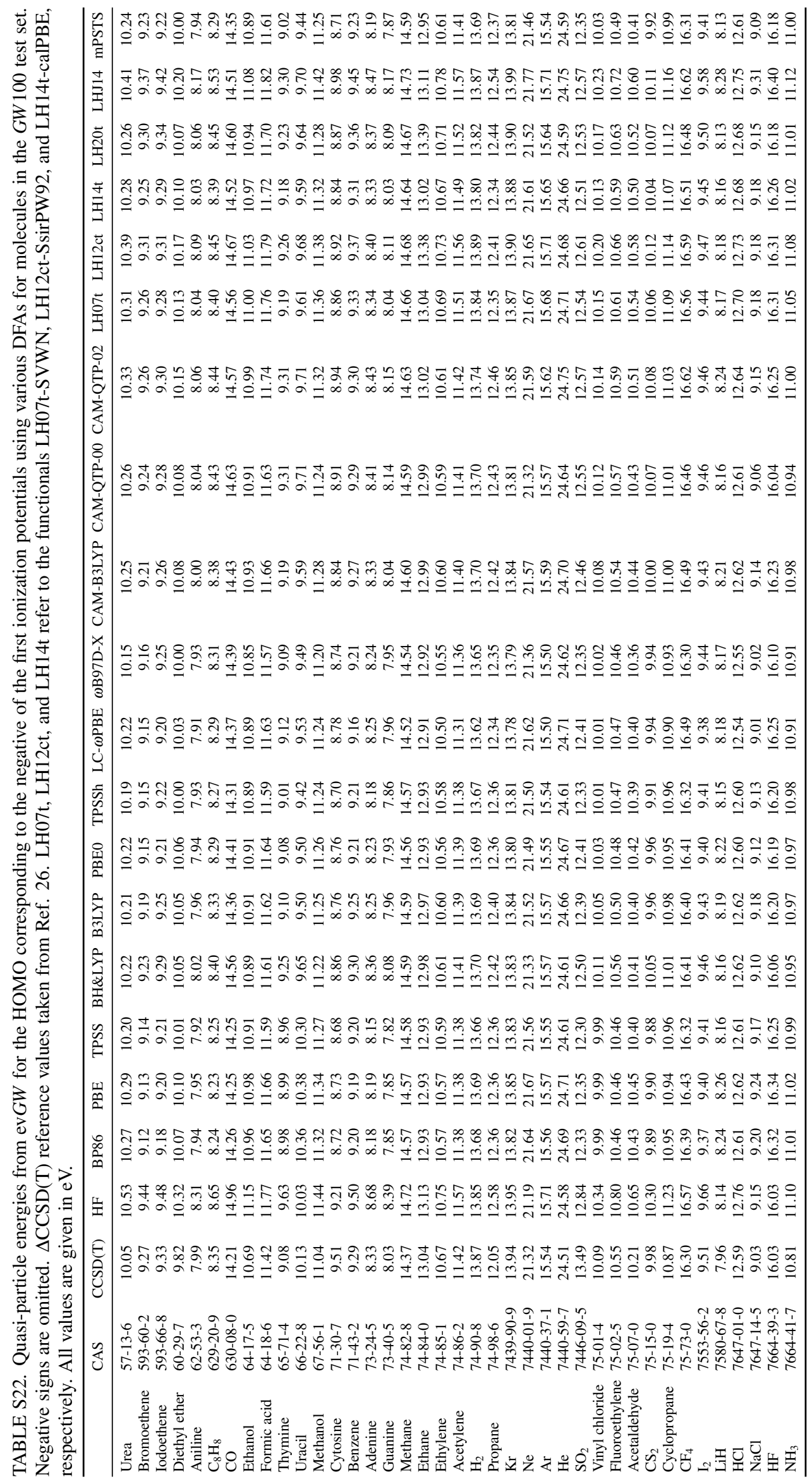




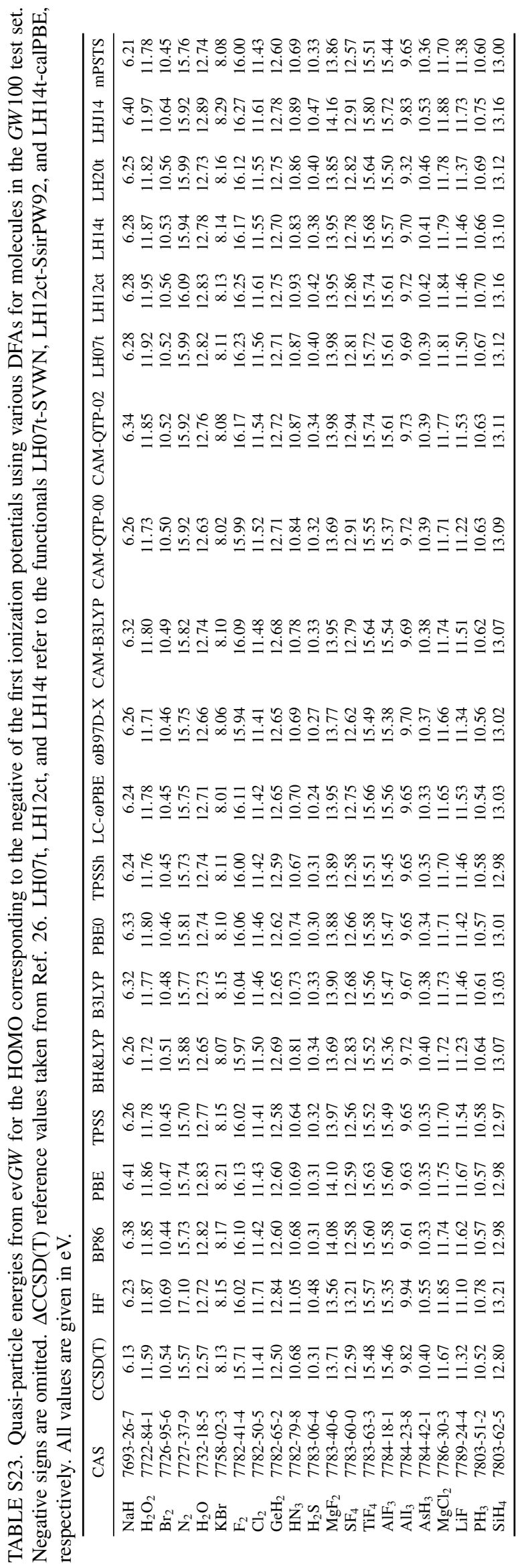




\section{S3. POLARIZABILITIES AND MAGNETIZABILITIES}

\section{A. First Test Set for Magnetizabilities and Polarizabilities}

This subsection lists the static dipole polarizability and magnetizability evaluated for the molecules $\mathrm{H}_{2} \mathrm{O}, \mathrm{NH}_{3}, \mathrm{CH}_{4}, \mathrm{PH}_{3}$, $\mathrm{H}_{2} \mathrm{~S}, \mathrm{CO}_{2}, \mathrm{CSO}, \mathrm{CS}_{2}, \mathrm{C}_{3} \mathrm{H}_{4}, \mathrm{C}_{2} \mathrm{H}_{2}, \mathrm{CH}_{2} \mathrm{O}, \mathrm{CF}_{2} \mathrm{O}, \mathrm{CH}_{4}$, and $\mathrm{HCN}$. Throughout this section the aug-cc-pV5Z basis set [3-5] is used. Polarizabilities and magnetizabilities were calculated at the molecular geometry optimized at the respective DFT/aug-ccpCV5Z level of theory. Tight thresholds were applied for the self-consistent field (SCF) procedure; energies were converged up to $10^{-9} \mathrm{E}_{\mathrm{h}}$ and a threshold of $10^{-9}$ a.u. was applied for the root mean square of the density matrix. Furthermore, the norm of the residuum in the coupled-perturbed Kohn-Sham (CPKS) equations was required to be less than $10^{-8}$ a.u. to ensure a well converged perturbed density matrix. Geometries were considered converged when the energy change between optimization steps falls below $10^{-6} \mathrm{E}_{\mathrm{h}}$. Very large grids (grid size 5) were employed for the numerical integration of the DFT parts [7]. To obtain the magnetizabilities, we first calculate the disturbed density matrices using TURBOMOLE [10-12, 43-45] These disturbed density matrices are then processed by the GIMIC program, [46-49] which calculates the gauge-invariant magnetizabilities from the current density susceptibility tensor [50]. The latter step is performed by an numerical integration, for which we employed the same grid (grid size 5) as within the TURBOMOLE calculations. As reference values for the magnetizabilities, we used the same values as Ruud, Helgaker, and co-workers [51-53]. For polarizabilities, we used experimental reference values. Were available, we used the same values as Hait and Head-Gordon [54]. For the compounds not found in the latter work, we used the experimental values from the $\mathrm{CRC}$ handbook of chemistry and physics [55]. The only exception is $\mathrm{CF}_{2} \mathrm{O}$, where no plausible experimental value could be found. Therefore, we estimated the polarizability from a CCSD calculation using the same basis set as described before.

While it was argued before that experimental data may be in error, we deemed them reliable enough for this overall assessment of polarizabilities. Unlike Ref. 54, we also update the geometry for each method. While this is rather time-consuming given the large basis set, the smaller test set makes it feasible. Our benchmarks for polarizabilities therefore try to simulate the deviation that is obtained if an experimental value is simulated in silico using only ab initio data from the given functional.

TABLE S24. Polarizabilitities of the test set evaluated for all tested functionals. LH07t, LH12ct, and LH14t refer to the functionals LH07tSVWN, LH12ct-SsirPW92, and LH14t-calPBE, respectively. All values in $\AA^{3}$.

\begin{tabular}{lccccccc}
\hline & Ref. & LH07t & LH12ct & LH14t & LH20t & mPSTS & LHJ14 \\
\hline $\mathrm{C}_{2} \mathrm{H}_{2}$ & 3.487 & 3.467 & 3.466 & 3.465 & 3.437 & 3.464 & 3.494 \\
$\mathrm{C}_{3} \mathrm{H}_{4}$ & 5.979 & 6.046 & 6.035 & 6.035 & 5.974 & 6.052 & 6.072 \\
$\mathrm{CF}_{2} \mathrm{O}$ & 2.811 & 2.754 & 2.718 & 2.755 & 2.701 & 2.802 & 2.862 \\
$\mathrm{CH}_{2} \mathrm{O}$ & 2.800 & 2.648 & 2.625 & 2.637 & 2.589 & 2.667 & 2.700 \\
$\mathrm{CH}_{4}$ & 2.448 & 2.508 & 2.498 & 2.494 & 2.457 & 2.493 & 2.522 \\
$\mathrm{CO}_{2}$ & 2.507 & 2.528 & 2.506 & 2.528 & 2.492 & 2.549 & 2.596 \\
$\mathrm{CS}_{2}$ & 8.201 & 8.236 & 8.247 & 8.243 & 8.220 & 8.146 & 8.246 \\
$\mathrm{CSO}$ & 5.085 & 5.055 & 5.046 & 5.059 & 5.023 & 5.035 & 5.100 \\
$\mathrm{H}_{2} \mathrm{O}$ & 1.501 & 1.446 & 1.420 & 1.445 & 1.413 & 1.472 & 1.517 \\
$\mathrm{H}_{2} \mathrm{~S}$ & 3.658 & 3.713 & 3.699 & 3.706 & 3.663 & 3.672 & 3.734 \\
$\mathrm{HCN}$ & 2.593 & 2.523 & 2.517 & 2.520 & 2.497 & 2.525 & 2.559 \\
$\mathrm{NH}_{3}$ & 2.103 & 2.138 & 2.110 & 2.133 & 2.095 & 2.154 & 2.205 \\
$\mathrm{PH}_{3}$ & 4.237 & 4.596 & 4.597 & 4.572 & 4.506 & 4.525 & 4.584 \\
\hline & & & & & & &
\end{tabular}




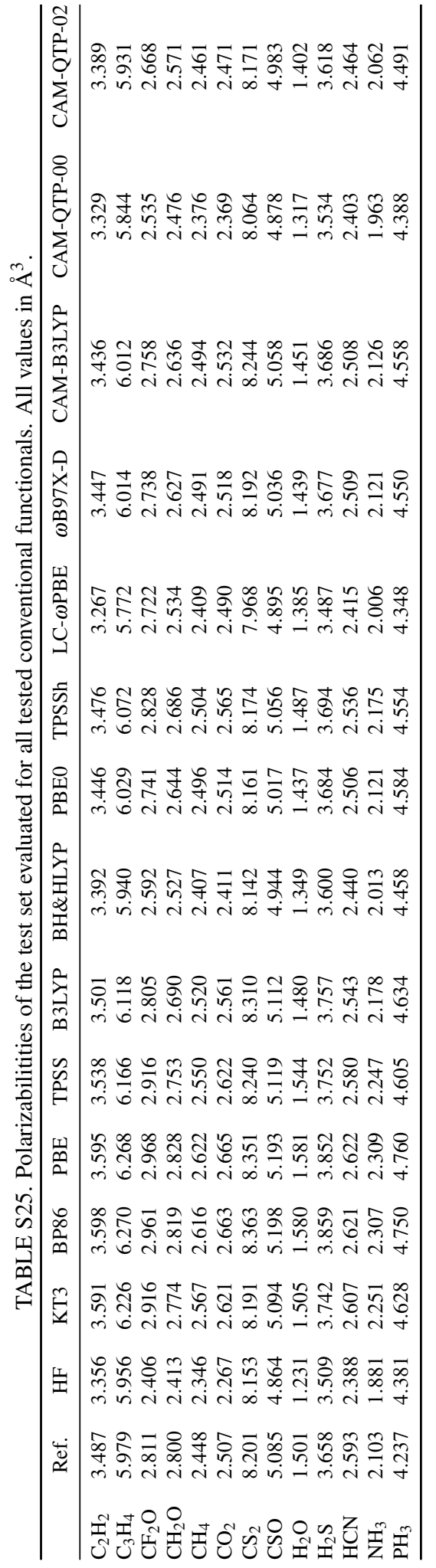




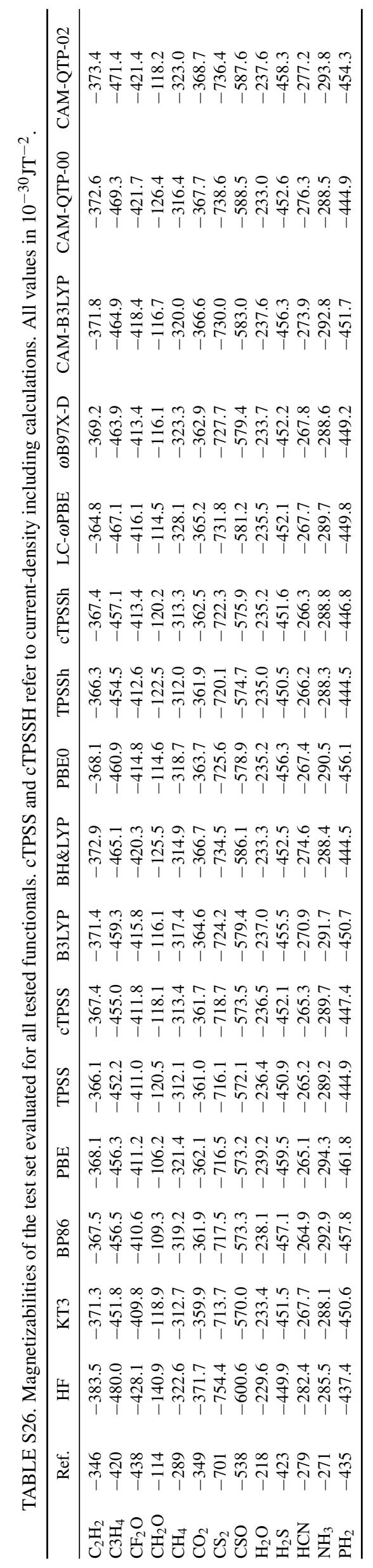




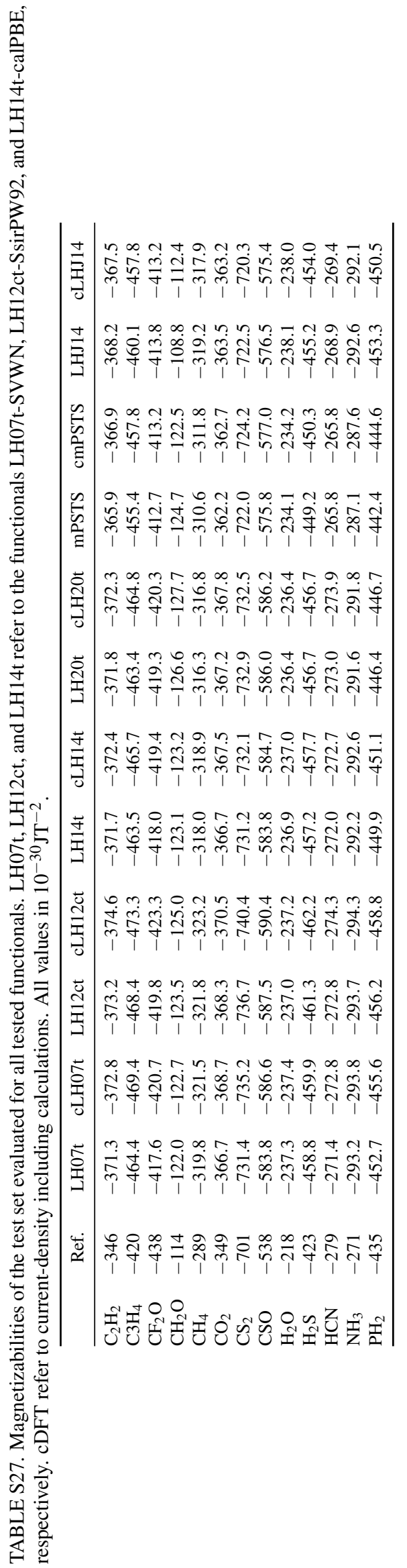




\section{B. Second Test Set for Magnetizabilities}

This subsection lists the static magnetizability evaluated for the test set reported in Ref. 56. We used the geometries provided within Ref. 56 and the same aug-cc-pCVQZ basis set [3-5, 57,-59], making results highly comparable. As before, tight thresholds were applied for the self-consistent field (SCF) procedure; energies were converged up to $10^{-9} \mathrm{E}_{\mathrm{h}}$ and a threshold of $10^{-9}$ a.u. was applied for the root mean square of the density matrix. Furthermore, the norm of the residuum in the coupledperturbed Kohn-Sham (CPKS) equations was required to be less than $10^{-8}$ a.u. to ensure a well converged perturbed density matrix. Note that $\mathrm{O}_{3}$ was excluded from the error analysis due to the CCSD(T) results probably still being not well converged [50, 56]. For conventional functionals our results are in excellent agreement with the those reported in Ref. [50, which was to be expected as the same procedure is employed.

Tab. S28 shows the MAE, MSD, and standard deviation for a the functional approximations tested within this work. The $\tau$-based LHFs perform rather well for the test set of Ref. 56, being among the best performing functionals among the 43 DFAs tested by Lehtola et al. for this set [50]. LH20t even manages to slightly surpass BH\&HLYP, the best performing global hybrid. Also, CAM-B3LYP, CAM-QTP-00, and CAM-QTP-02 perform rather well. To conclude, DFAs which incorporate a significant amount of HF exchange perform rather well for this set set, which carries over to the $\tau$-dependent LHFs. This behavior has already been observed for excitation energies and $G_{0} W_{0}$ quasiparticle energies. The mPSTS performance is similar to TPSS(h), which is again in line with previous observations. Within the GIAO formalism used in this work, including current-dependent terms is only of minor importance for the small molecules investigated. Interestingly, including the current-depenent terms improves the accuracy of TPSS and the LHF mPSTS slightly, while deteriorating the results of TPSSh. While not included in the statistical analysis, a close inspection of Tab. S29 reveals that large effects of the current density density are found for $\mathrm{O}_{3}$ for TPSS(h), mPSTS, LH20t, and LHJ14, indicating that the inclusion of these terms is important for these functionals in some cases. We also note that these terms are detrimental for full gauge-invariance, and therefore a more significant decline in quality may be observed for larger molecules.

TABLE S28. Mean absolute percent-wise error (MAPE), mean signed percent-wise deviation (MSPD), and mean standard deviation for magnetizabilities in the second test set. All calculation were performed using the aug-cc-pCVQZ basis set to guarantee a converged result.

\begin{tabular}{lrrr}
\hline & \multicolumn{3}{c}{ Magnetizability } \\
\cline { 2 - 4 } & MAE & \multicolumn{1}{c}{ MSD } & STD \\
\hline HF & 7.22 & -3.70 & 8.41 \\
KT3 & 9.20 & 8.40 & 8.04 \\
BP86 & 8.70 & 7.49 & 8.78 \\
PBE & 9.15 & 7.09 & 9.45 \\
TPSS & 8.24 & 7.88 & 6.90 \\
cTPSS & 7.83 & 7.46 & 7.07 \\
BH\&HLYP & 3.13 & 2.17 & 4.70 \\
B3LYP & 5.44 & 4.55 & 6.04 \\
PBE0 & 5.98 & 5.59 & 6.86 \\
TPSSh & 7.22 & 7.10 & 6.08 \\
cTPSSh & 7.67 & 7.58 & 8.12 \\
LC- $\omega$ PBE & 4.96 & 4.15 & 6.15 \\
WB97X-D & 6.27 & 5.94 & 6.45 \\
CAM-B3LYP & 3.74 & 2.41 & 4.90 \\
CAM-QTP-00 & 3.24 & 0.91 & 4.72 \\
CAM-QTP-02 & 3.29 & -0.20 & 4.40 \\
LH07t & 3.68 & 2.01 & 4.81 \\
cLH07t & 3.55 & 0.18 & 4.55 \\
LH12ct & 3.59 & 0.14 & 4.71 \\
cLH12ct & 3.74 & -1.89 & 4.48 \\
LH14t & 3.21 & 2.06 & 4.29 \\
cLH14t & 3.02 & 1.28 & 4.14 \\
LH20t & 2.70 & 1.14 & 4.17 \\
cLH20t & 2.47 & 0.45 & 3.77 \\
mPSTS & 7.15 & 7.13 & 6.10 \\
cmPSTS & 6.85 & 6.83 & 6.39 \\
LHJ14 & 6.98 & 5.62 & 7.09 \\
cLHJ14 & 7.13 & 5.56 & 7.70 \\
\hline & & &
\end{tabular}




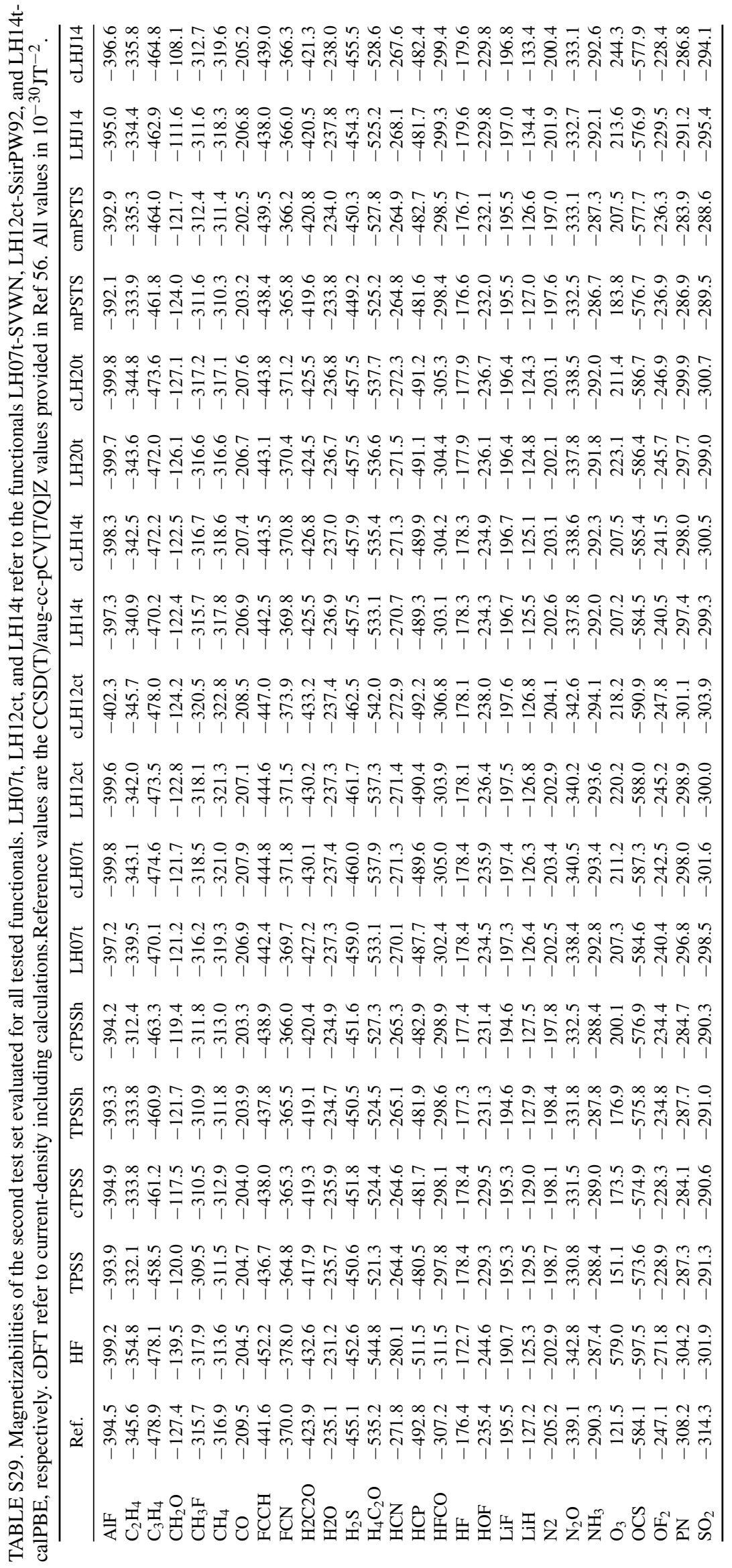




\section{S4. NMR COUPLING DATA}

The structures of the molecules are obtained using the aug-cc-pVQZ basis set [3, 4, 60] and large grids (gridsize 4) [7]. Energies were converged with a criterion of $10^{-9} \mathrm{E}_{\mathrm{h}}$ for the SCF energy and $10^{-9}$ a.u. for the root mean square of the density matrix. Geometries were considered converged when the energy change between optimization steps falls below $10^{-6} E_{h}$. The Cartesian coordinates of the optimized structures are listed after the NMR spin-spin coupling constants in Tab. S30. These were calculated with the aug-ccJ-pVTZ basis set. The ccJ-pVTZ [61] basis set was obtained from the Basis Set Exchange library [62, 63] and the augmentation functions were added [4]. This allows to compare the DFT results to the CC3 results [64]. All four terms of Ramsey's theory [65], i.e. the Fermi-contact (FC), spin-dipole (SD), paramagnetic spin-orbit (PSO), and the diamagnetic spin-orbit (DSO) term, were calculated. The norm of the residuum in the coupled perturbed Kohn-Sham equations [6] was required to be less than $10^{-6}$ a.u. and due to the generalized kinetic energy density [8] the PSO term was also calculated iteratively for TPSS. Note that the previous implementation of spin-spin coupling constants at the meta-generalized gradient approximation and LHF level of theory [66] neglected the paramagnetic current density contribution to the exchange-correlation kernel. All calculations in this work were carried out with TURBOMOLE [6, 10, 14]. 


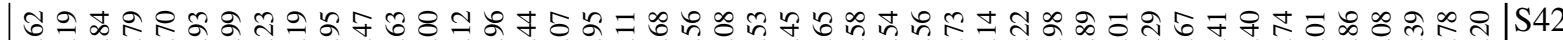

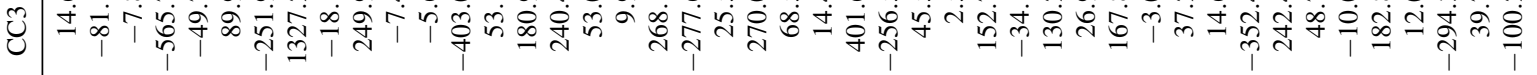

林

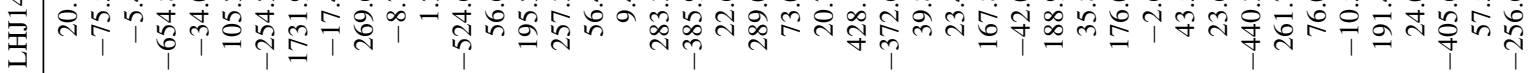

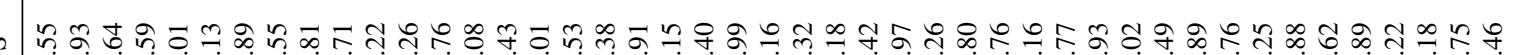

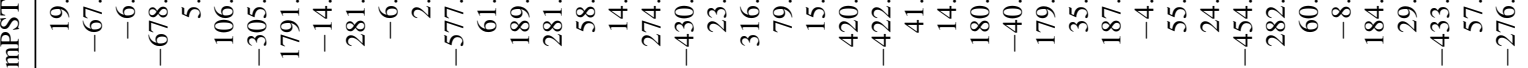

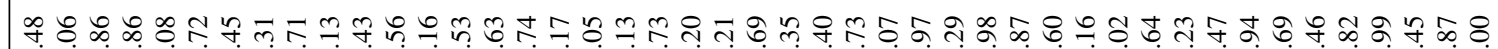

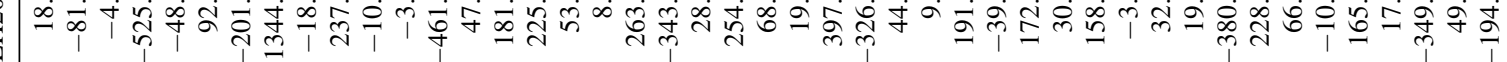

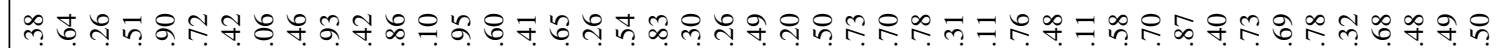

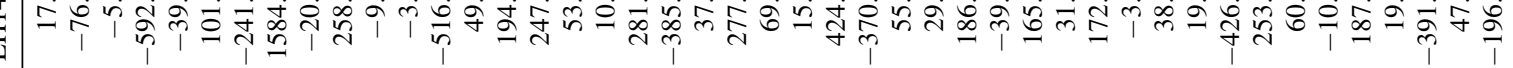

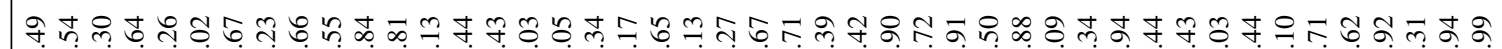
तु

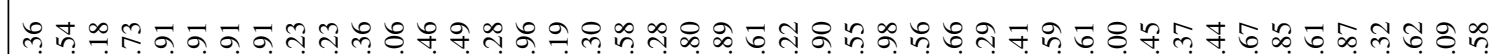

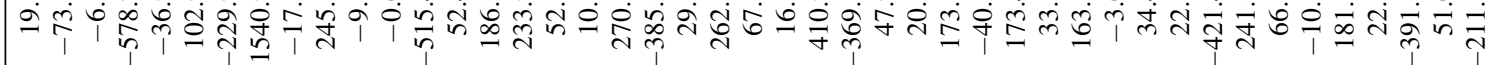

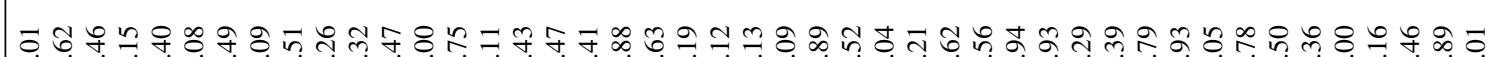

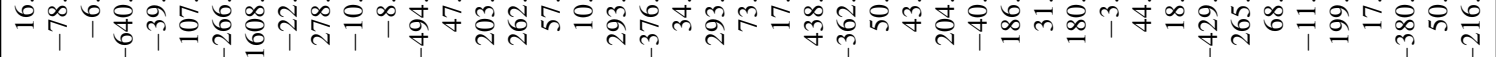

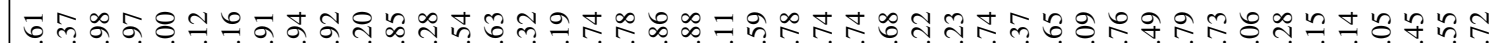

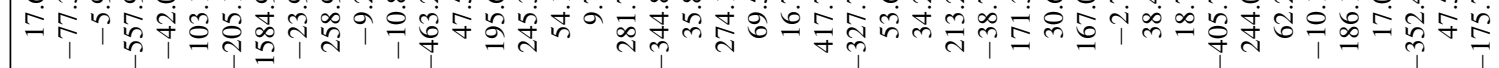

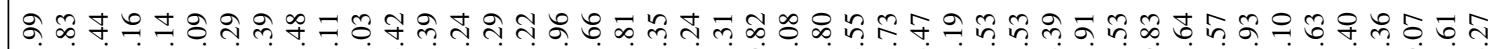

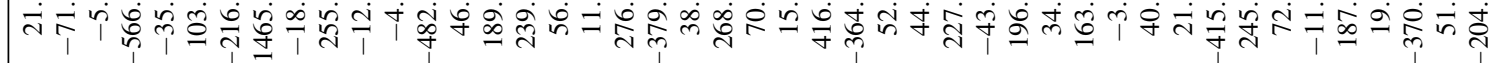

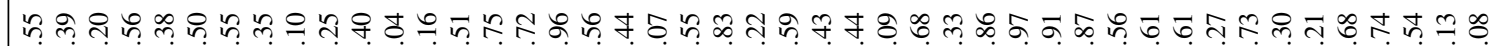
तेंत

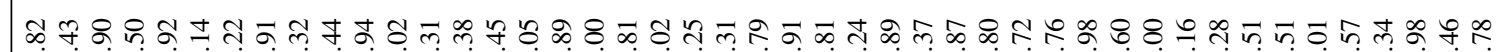

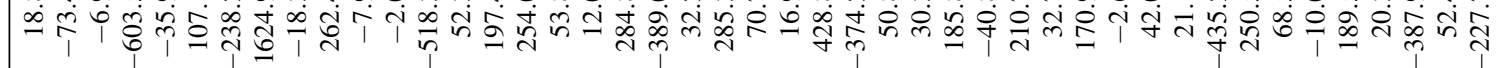

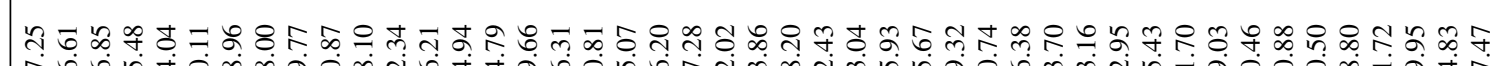
=

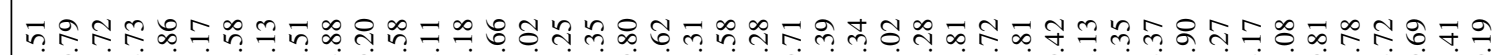

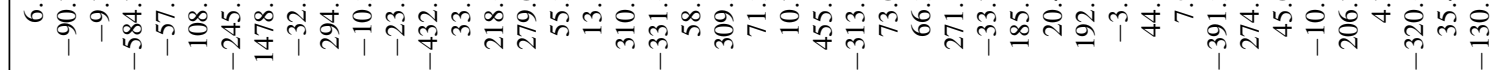

击

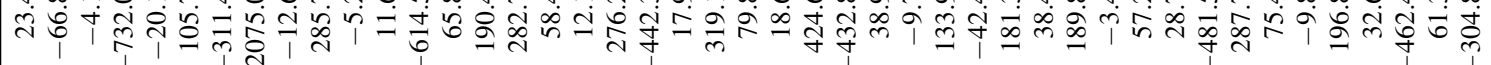
的 人

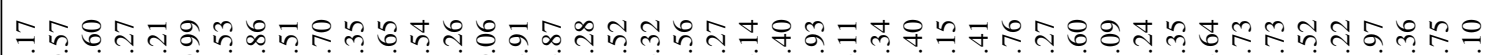

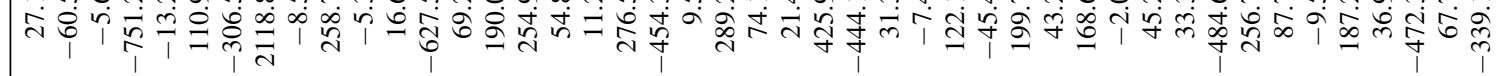

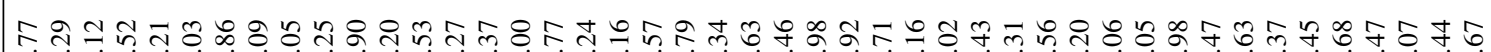

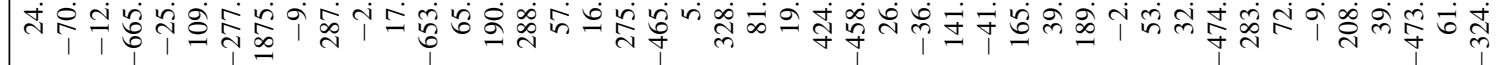




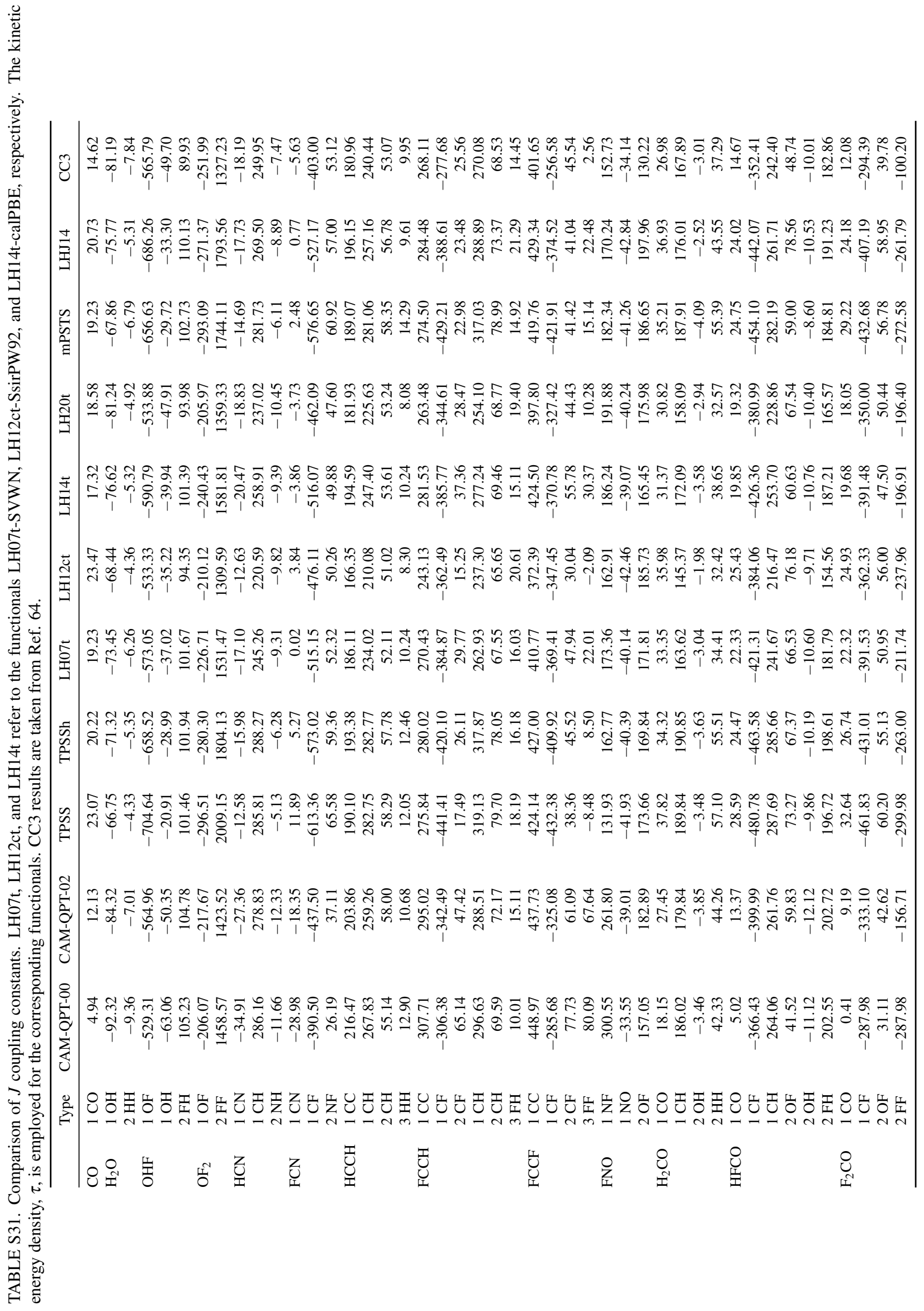


1. Optimized Structures at the KT3 Level

The Cartesian coordinates of the structures are listed in Ångström.

\begin{tabular}{|c|c|c|c|}
\hline C & -0.0000000 & -0.0000000 & -0.5660329 \\
\hline 0 & 0.0000000 & 0.0000000 & 0.5660329 \\
\hline $\mathrm{C}$ & 0.0000000 & 0.0000000 & 0.0966417 \\
\hline 0 & 0.0000000 & 0.0000000 & 1.2720973 \\
\hline $\mathrm{F}$ & 1.0647210 & 0.0000000 & -0.6843695 \\
\hline $\mathrm{F}$ & -1.0647210 & 0.0000000 & -0.6843695 \\
\hline C & 0.0000000 & -0.0000000 & 0.5943687 \\
\hline $\mathrm{C}$ & 0.0000000 & 0.0000000 & -0.5943687 \\
\hline $\mathrm{F}$ & 0.0000000 & 0.0000000 & 1.8776851 \\
\hline $\mathrm{F}$ & -0.0000000 & 0.0000000 & -1.8776851 \\
\hline $\mathrm{C}$ & -0.0000000 & 0.0000000 & 0.6547277 \\
\hline C & -0.0000000 & 0.0000000 & -0.5424281 \\
\hline $\mathrm{H}$ & 0.0000000 & 0.0000000 & 1.7060317 \\
\hline $\mathrm{F}$ & 0.0000000 & 0.0000000 & -1.8183313 \\
\hline $\mathrm{F}$ & -0.0000000 & 0.0000000 & -1.2315355 \\
\hline $\mathrm{C}$ & 0.0000000 & 0.0000000 & 0.0355685 \\
\hline N & -0.0000000 & -0.0000000 & 1.1959670 \\
\hline 0 & -0.9973250 & 0.3268177 & 0.0000000 \\
\hline $\mathrm{N}$ & 0.1237154 & 0.5016792 & 0.0000000 \\
\hline $\mathrm{F}$ & 0.8736096 & -0.8284969 & 0.0000000 \\
\hline C & 0.0000000 & 0.0000000 & -0.0104035 \\
\hline 0 & 0.0000000 & 0.0000000 & 1.1925244 \\
\hline $\mathrm{H}$ & 0.9319701 & 0.0000000 & -0.5910604 \\
\hline $\mathrm{H}$ & -0.9319701 & 0.0000000 & -0.5910604 \\
\hline 0 & 0.0000000 & 0.0000000 & 0.3928403 \\
\hline $\mathrm{H}$ & -0.7498634 & 0.0000000 & -0.1964207 \\
\hline $\mathrm{H}$ & 0.7498634 & 0.0000000 & -0.1964207 \\
\hline $\mathrm{C}$ & -0.0000000 & 0.0000000 & 0.6006333 \\
\hline $\mathrm{C}$ & -0.0000000 & 0.0000000 & -0.6006333 \\
\hline $\mathrm{H}$ & 0.0000000 & 0.0000000 & 1.6541275 \\
\hline $\mathrm{H}$ & 0.0000000 & -0.0000000 & -1.6541275 \\
\hline $\mathrm{H}$ & -0.0000000 & 0.0000000 & -1.0901359 \\
\hline $\mathrm{C}$ & 0.0000000 & 0.0000000 & -0.0315951 \\
\hline $\mathrm{N}$ & -0.0000000 & 0.0000000 & 1.1217310 \\
\hline
\end{tabular}




$\begin{array}{rrrr}\text { C } & 0.0720472 & 0.0550483 & 0.0000000 \\ \text { O } & 1.2169233 & -0.2299166 & 0.0000000 \\ \text { F } & -0.9136128 & -0.8686941 & 0.0000000 \\ \text { H } & -0.3753577 & 1.0435623 & 0.0000000 \\ & & & \\ \text { O } & 0.0000000 & 0.0000000 & 0.5748528 \\ F & -1.1104875 & 0.0000000 & -0.2874264 \\ F & 1.1104875 & 0.0000000 & -0.2874264 \\ & & & \\ & & & \\ \text { O } & -0.5068193 & 0.1803781 & 0.0000000 \\ \text { H } & -0.3585553 & -0.7709927 & 0.0000000 \\ \text { F } & 0.8653747 & 0.5906146 & 0.0000000\end{array}$




\section{Optimized Structures at the BP86 Level}

The Cartesian coordinates of the structures are listed in Ångström.

\begin{tabular}{|c|c|c|c|}
\hline C & 0.0000000 & 0.0000000 & -0.5676693 \\
\hline 0 & -0.0000000 & 0.0000000 & 0.5676693 \\
\hline C & 0.0000000 & 0.0000000 & 0.0987624 \\
\hline 0 & 0.0000000 & 0.0000000 & 1.2792126 \\
\hline $\mathrm{F}$ & 1.0739031 & 0.0000000 & -0.6889875 \\
\hline $\mathrm{F}$ & -1.0739031 & 0.0000000 & -0.6889875 \\
\hline C & 0.0000000 & 0.0000000 & 0.5975042 \\
\hline C & 0.0000000 & 0.0000000 & -0.5975033 \\
\hline $\mathrm{F}$ & -0.0000000 & -0.0000000 & 1.8884892 \\
\hline $\mathrm{F}$ & -0.0000000 & -0.0000000 & -1.8884902 \\
\hline C & 0.0000000 & -0.0000000 & 0.6553029 \\
\hline C & -0.0000000 & -0.0000000 & -0.5472193 \\
\hline $\mathrm{H}$ & -0.0000000 & 0.0000000 & 1.7223915 \\
\hline $\mathrm{F}$ & 0.0000000 & 0.0000000 & -1.8304752 \\
\hline $\mathrm{F}$ & 0.0000000 & 0.0000000 & -1.2371752 \\
\hline C & -0.0000000 & -0.0000000 & 0.0365798 \\
\hline $\mathrm{N}$ & 0.0000000 & 0.0000000 & 1.2005954 \\
\hline 0 & -1.0065778 & 0.3335446 & 0.0000000 \\
\hline $\mathrm{N}$ & 0.1165349 & 0.5120554 & 0.0000000 \\
\hline $\mathrm{F}$ & 0.8900429 & -0.8456000 & 0.0000000 \\
\hline $\mathrm{C}$ & -0.0000000 & 0.0000000 & -0.0058311 \\
\hline 0 & 0.0000000 & 0.0000000 & 1.2021378 \\
\hline $\mathrm{H}$ & 0.9466826 & 0.0000000 & -0.5981534 \\
\hline $\mathrm{H}$ & -0.9466826 & 0.0000000 & -0.5981534 \\
\hline 0 & 0.0000000 & 0.0000000 & 0.3969404 \\
\hline $\mathrm{H}$ & -0.7650283 & 0.0000000 & -0.1984707 \\
\hline $\mathrm{H}$ & 0.7650283 & 0.0000000 & -0.198470 \\
\hline C & -0.0000000 & -0.0000000 & 0.6029673 \\
\hline C & -0.0000000 & 0.0000000 & -0.6029681 \\
\hline $\mathrm{H}$ & 0.0000000 & -0.0000000 & 1.6722451 \\
\hline $\mathrm{H}$ & -0.0000000 & 0.0000000 & -1.6722443 \\
\hline $\mathrm{H}$ & 0.0000000 & 0.0000000 & -1.1016281 \\
\hline C & -0.0000000 & 0.0000000 & -0.0276517 \\
\hline & -0.0000000 & 0.0000000 & 1.1292797 \\
\hline
\end{tabular}




$\begin{array}{rrrr}\text { C } & 0.0753348 & 0.0538845 & 0.0000000 \\ \text { O } & 1.2249044 & -0.2331981 & 0.0000000 \\ \text { F } & -0.9204369 & -0.8787948 & 0.0000000 \\ \text { H } & -0.3798023 & 1.0581083 & 0.0000000 \\ & & & \\ & & & \\ O & 0.0000000 & 0.0000000 & 0.5790173 \\ F & -1.1257032 & 0.0000000 & -0.2895086 \\ F & 1.1257032 & 0.0000000 & -0.2895086 \\ & & & \\ & & & \\ 0 & -0.5113929 & 0.1851137 & 0.0000000 \\ H & -0.3639202 & -0.7838284 & 0.0000000 \\ F & 0.8753131 & 0.5987147 & 0.0000000\end{array}$




\section{Optimized Structures at the PBE Level}

The Cartesian coordinates of the structures are listed in Ångström.

\begin{tabular}{|c|c|c|c|}
\hline C & -0.0000000 & 0.0000000 & -0.5677214 \\
\hline 0 & 0.0000000 & 0.0000000 & 0.5677214 \\
\hline C & 0.0000000 & 0.0000000 & 0.0984971 \\
\hline 0 & 0.0000000 & 0.0000000 & 1.2790630 \\
\hline$F$ & 1.0733114 & 0.0000000 & -0.6887800 \\
\hline $\mathrm{F}$ & -1.0733114 & 0.0000000 & -0.6887800 \\
\hline $\mathrm{C}$ & -0.0000000 & -0.0000000 & 0.5978651 \\
\hline $\mathrm{C}$ & -0.0000000 & -0.0000000 & -0.5978637 \\
\hline $\mathrm{F}$ & 0.0000000 & 0.0000000 & 1.8880997 \\
\hline $\mathrm{F}$ & -0.0000000 & 0.0000000 & -1.8881011 \\
\hline C & -0.0000000 & 0.0000000 & 0.6552887 \\
\hline $\mathrm{C}$ & -0.0000000 & -0.0000000 & -0.5479585 \\
\hline $\mathrm{H}$ & 0.0000000 & 0.0000000 & 1.7231452 \\
\hline $\mathrm{F}$ & 0.0000000 & 0.0000000 & -1.8304754 \\
\hline F & 0.0000000 & 0.0000000 & -1.2369375 \\
\hline C & -0.0000000 & 0.0000000 & 0.0361987 \\
\hline $\mathrm{N}$ & 0.0000000 & -0.0000000 & 1.2007388 \\
\hline 0 & -1.0057419 & 0.3327849 & 0.0000000 \\
\hline $\mathrm{N}$ & 0.1174630 & 0.5107690 & 0.0000000 \\
\hline $\mathrm{F}$ & 0.8882789 & -0.8435540 & 0.0000000 \\
\hline $\mathrm{C}$ & -0.0000000 & 0.0000000 & -0.0056613 \\
\hline 0 & 0.0000000 & 0.0000000 & 1.2021953 \\
\hline $\mathrm{H}$ & 0.9472918 & 0.0000000 & -0.5982670 \\
\hline $\mathrm{H}$ & -0.9472918 & 0.0000000 & -0.5982670 \\
\hline 0 & 0.0000000 & 0.0000000 & 0.396903 \\
\hline $\mathrm{H}$ & -0.7646875 & 0.0000000 & -0.198452 \\
\hline $\mathrm{H}$ & 0.7646875 & 0.0000000 & -0.198452 \\
\hline C & -0.0000000 & 0.0000000 & 0.6033272 \\
\hline C & 0.0000000 & 0.0000000 & -0.6033280 \\
\hline $\mathrm{H}$ & -0.0000000 & 0.0000000 & 1.673439 \\
\hline $\mathrm{H}$ & -0.0000000 & 0.0000000 & -1.673439 \\
\hline $\mathrm{H}$ & 0.0000000 & -0.0000000 & -1.1023967 \\
\hline C & -0.0000000 & 0.0000000 & -0.027552 \\
\hline & 0.0000000 & 0.0000000 & 1.129949 \\
\hline
\end{tabular}




$\begin{array}{rrrr}\text { C } & 0.0749982 & 0.0532876 & 0.0000000 \\ \text { O } & 1.2247941 & -0.2335303 & 0.0000000 \\ \text { F } & -0.9196470 & -0.8782799 & 0.0000000 \\ \text { H } & -0.3801453 & 1.0585226 & 0.0000000 \\ & & & \\ & & & \\ O & 0.0000000 & 0.0000000 & 0.5785357 \\ F & -1.1232484 & 0.0000000 & -0.2892678 \\ F & 1.1232484 & 0.0000000 & -0.2892678 \\ & & & \\ & & & \\ 0 & -0.5100930 & 0.1853243 & 0.0000000 \\ \text { H } & -0.3639479 & -0.7836332 & 0.0000000 \\ \text { F } & 0.8740409 & 0.5983089 & 0.0000000\end{array}$




\section{Optimized Structures at the TPSS Level}

The Cartesian coordinates of the structures are listed in Ångström.

\begin{tabular}{|c|c|c|c|}
\hline $\mathrm{C}$ & 0.0000000 & 0.0000000 & -0.5668147 \\
\hline 0 & 0.0000000 & 0.0000000 & 0.5668147 \\
\hline C & 0.0000000 & 0.0000000 & 0.0991696 \\
\hline 0 & 0.0000000 & 0.0000000 & 1.2776498 \\
\hline$F$ & 1.0716881 & 0.0000000 & -0.6884097 \\
\hline $\mathrm{F}$ & -1.0716881 & 0.0000000 & -0.6884097 \\
\hline $\mathrm{C}$ & -0.0000000 & -0.0000000 & 0.5951832 \\
\hline $\mathrm{C}$ & -0.0000000 & 0.0000000 & -0.5951836 \\
\hline$F$ & 0.0000000 & 0.0000000 & 1.8861263 \\
\hline $\mathrm{F}$ & -0.0000000 & -0.0000000 & -1.8861259 \\
\hline C & -0.0000000 & -0.0000000 & 0.6543148 \\
\hline $\mathrm{C}$ & -0.0000000 & 0.0000000 & -0.5439198 \\
\hline $\mathrm{H}$ & 0.0000000 & -0.0000000 & 1.7170946 \\
\hline $\mathrm{F}$ & 0.0000000 & -0.0000000 & -1.8274896 \\
\hline F & -0.0000000 & 0.0000000 & -1.2359851 \\
\hline $\mathrm{C}$ & 0.0000000 & -0.0000000 & 0.0374307 \\
\hline $\mathrm{N}$ & -0.0000000 & 0.0000000 & 1.1985544 \\
\hline 0 & -1.0046225 & 0.3325474 & 0.0000000 \\
\hline $\mathrm{N}$ & 0.1161822 & 0.5127475 & 0.0000000 \\
\hline $\mathrm{F}$ & 0.8884404 & -0.8452949 & 0.0000000 \\
\hline C & -0.0000000 & 0.0000000 & -0.0082642 \\
\hline 0 & 0.0000000 & 0.0000000 & 1.1992331 \\
\hline $\mathrm{H}$ & 0.9416390 & 0.0000000 & -0.5954844 \\
\hline $\mathrm{H}$ & -0.9416390 & 0.0000000 & -0.5954844 \\
\hline 0 & 0.0000000 & 0.0000000 & 0.3958577 \\
\hline $\mathrm{H}$ & -0.7641956 & 0.0000000 & -0.1979294 \\
\hline $\mathrm{H}$ & 0.7641956 & -0.0000000 & -0.1979294 \\
\hline C & 0.0000000 & 0.0000000 & 0.6010033 \\
\hline C & 0.0000000 & 0.0000000 & -0.6010040 \\
\hline $\mathrm{H}$ & -0.0000000 & -0.0000000 & 1.6658711 \\
\hline $\mathrm{H}$ & -0.0000000 & -0.0000000 & -1.665870 \\
\hline $\mathrm{H}$ & 0.0000000 & 0.0000000 & -1.0977404 \\
\hline $\mathrm{C}$ & -0.0000000 & 0.0000000 & -0.0282215 \\
\hline & 0.0000000 & -0.0000000 & 1.1259618 \\
\hline
\end{tabular}




$\begin{array}{rrrr}\text { C } & 0.0747698 & 0.0559192 & 0.0000000 \\ \text { O } & 1.2224894 & -0.2326042 & 0.0000000 \\ \text { F } & -0.9189884 & -0.8770887 & 0.0000000 \\ \text { H } & -0.3782708 & 1.0537737 & 0.0000000 \\ & & & \\ \text { O } & 0.0000000 & 0.0000000 & 0.5808264 \\ F & -1.1254032 & 0.0000000 & -0.2904132 \\ \text { F } & 1.1254032 & 0.0000000 & -0.2904132 \\ & & & \\ & & & \\ \text { O } & -0.5130506 & 0.1840918 & 0.0000000 \\ \text { H } & -0.3630472 & -0.7824067 & 0.0000000 \\ \text { F } & 0.8760978 & 0.5983149 & 0.0000000\end{array}$


5. Optimized Structures at the BH\&HLYP Level

The Cartesian coordinates of the structures are listed in Ångström.

\begin{tabular}{|c|c|c|c|}
\hline C & -0.0000000 & -0.0000000 & -0.5555617 \\
\hline 0 & 0.0000000 & 0.0000000 & 0.5555617 \\
\hline C & 0.0000000 & 0.0000000 & 0.0912191 \\
\hline 0 & 0.0000000 & 0.0000000 & 1.2489920 \\
\hline $\mathrm{F}$ & 1.0495062 & 0.0000000 & -0.6701056 \\
\hline $\mathrm{F}$ & -1.0495062 & 0.0000000 & -0.6701056 \\
\hline C & -0.0000000 & 0.0000000 & 0.5845265 \\
\hline C & -0.0000000 & 0.0000000 & -0.5845264 \\
\hline $\mathrm{F}$ & 0.0000000 & -0.0000000 & 1.8549349 \\
\hline $\mathrm{F}$ & 0.0000000 & 0.0000000 & -1.8549349 \\
\hline C & 0.0000000 & 0.0000000 & 0.6419113 \\
\hline $\mathrm{C}$ & 0.0000000 & 0.0000000 & -0.5367754 \\
\hline $\mathrm{H}$ & -0.0000000 & 0.0000000 & 1.6948704 \\
\hline $\mathrm{F}$ & -0.0000000 & -0.0000000 & -1.8000063 \\
\hline F & 0.0000000 & 0.0000000 & -1.2113147 \\
\hline C & -0.0000000 & 0.0000000 & 0.0383811 \\
\hline $\mathrm{N}$ & -0.0000000 & 0.0000000 & 1.1729336 \\
\hline 0 & -0.9702515 & 0.3121282 & 0.0000000 \\
\hline $\mathrm{N}$ & 0.1329594 & 0.4802608 & 0.0000000 \\
\hline $\mathrm{F}$ & 0.8372921 & -0.7923890 & 0.0000000 \\
\hline C & -0.0000000 & 0.0000000 & -0.0069424 \\
\hline 0 & 0.0000000 & 0.0000000 & 1.1787926 \\
\hline $\mathrm{H}$ & 0.9299905 & 0.0000000 & -0.5859251 \\
\hline $\mathrm{H}$ & -0.9299905 & 0.0000000 & -0.5859251 \\
\hline 0 & 0.0000000 & 0.0000000 & 0.3817558 \\
\hline $\mathrm{H}$ & -0.7568739 & 0.0000000 & -0.1908785 \\
\hline $\mathrm{H}$ & 0.7568739 & -0.0000000 & -0.1908785 \\
\hline C & -0.0000000 & 0.0000000 & 0.5924332 \\
\hline C & 0.0000000 & 0.0000000 & -0.5924337 \\
\hline $\mathrm{H}$ & -0.0000000 & 0.0000000 & 1.6472147 \\
\hline $\mathrm{H}$ & -0.0000000 & 0.0000000 & -1.647214 \\
\hline $\mathrm{H}$ & 0.0000000 & 0.0000000 & -1.0830148 \\
\hline $\mathrm{C}$ & -0.0000000 & -0.0000000 & -0.0247135 \\
\hline & 0.0000000 & 0.0000000 & 1.1077284 \\
\hline
\end{tabular}




$\begin{array}{rrrr}\text { C } & 0.0668436 & 0.0485333 & 0.0000000 \\ \text { O } & 1.1967561 & -0.2294642 & 0.0000000 \\ \text { F } & -0.8941907 & -0.8609447 & 0.0000000 \\ \text { H } & -0.3694090 & 1.0418756 & 0.0000000 \\ & & & \\ \text { O } & 0.0000000 & 0.0000000 & 0.5627949 \\ F & -1.0704683 & 0.0000000 & -0.2813975 \\ F & 1.0704683 & 0.0000000 & -0.2813975 \\ & & & \\ & & & \\ \text { O } & -0.4838116 & 0.1804798 & 0.0000000 \\ \text { H } & -0.3626757 & -0.7684626 & 0.0000000 \\ \text { F } & 0.8464873 & 0.5879828 & 0.0000000\end{array}$


6. Optimized Structures at the B3LYP Level

The Cartesian coordinates of the structures are listed in Ångström.

\begin{tabular}{|c|c|c|c|}
\hline C & 0.0000000 & 0.0000000 & -0.5620164 \\
\hline 0 & -0.0000000 & 0.0000000 & 0.5620164 \\
\hline C & 0.0000000 & 0.0000000 & 0.0962531 \\
\hline 0 & 0.0000000 & 0.0000000 & 1.2661732 \\
\hline $\mathrm{F}$ & 1.0649192 & 0.0000000 & -0.6812132 \\
\hline $\mathrm{F}$ & -1.0649192 & 0.0000000 & -0.6812132 \\
\hline C & -0.0000000 & 0.0000000 & 0.5912988 \\
\hline $\mathrm{C}$ & -0.0000000 & 0.0000000 & -0.5912978 \\
\hline $\mathrm{F}$ & 0.0000000 & 0.0000000 & 1.8755818 \\
\hline $\mathrm{F}$ & 0.0000000 & 0.0000000 & -1.8755827 \\
\hline C & 0.0000000 & 0.0000000 & 0.6498015 \\
\hline C & 0.0000000 & 0.0000000 & -0.5412674 \\
\hline $\mathrm{H}$ & -0.0000000 & 0.0000000 & 1.7096099 \\
\hline $\mathrm{F}$ & -0.0000000 & -0.0000000 & -1.8181440 \\
\hline $\mathrm{F}$ & 0.0000000 & 0.0000000 & -1.2265954 \\
\hline C & 0.0000000 & 0.0000000 & 0.0383552 \\
\hline $\mathrm{N}$ & -0.0000000 & 0.0000000 & 1.1882402 \\
\hline 0 & -0.9915621 & 0.3257321 & 0.0000000 \\
\hline $\mathrm{N}$ & 0.1192014 & 0.5036793 & 0.0000000 \\
\hline $\mathrm{F}$ & 0.8723606 & -0.8294115 & 0.0000000 \\
\hline C & 0.0000000 & 0.0000000 & -0.0068583 \\
\hline 0 & 0.0000000 & 0.0000000 & 1.1918465 \\
\hline $\mathrm{H}$ & 0.9382399 & 0.0000000 & -0.5924941 \\
\hline $\mathrm{H}$ & -0.9382399 & -0.0000000 & -0.5924941 \\
\hline 0 & 0.0000000 & 0.0000000 & 0.3894532 \\
\hline $\mathrm{H}$ & -0.7628538 & 0.0000000 & -0.1947272 \\
\hline $\mathrm{H}$ & 0.7628538 & 0.0000000 & -0.1947272 \\
\hline C & 0.0000000 & 0.0000000 & 0.5979241 \\
\hline C & 0.0000000 & 0.0000000 & -0.5979245 \\
\hline $\mathrm{H}$ & -0.0000000 & 0.0000000 & 1.6596383 \\
\hline $\mathrm{H}$ & 0.0000000 & 0.0000000 & -1.6596379 \\
\hline $\mathrm{H}$ & 0.0000000 & -0.0000000 & -1.0922968 \\
\hline $\mathrm{C}$ & -0.0000000 & 0.0000000 & -0.0265314 \\
\hline $\mathrm{N}$ & 0.0000000 & -0.0000000 & 1.1188282 \\
\hline
\end{tabular}




$\begin{array}{rrrr}\text { C } & 0.0723046 & 0.0525156 & 0.0000000 \\ \text { O } & 1.2127000 & -0.2311684 & 0.0000000 \\ \text { F } & -0.9102505 & -0.8719740 & 0.0000000 \\ H & -0.3747541 & 1.0506268 & 0.0000000 \\ & & & \\ & & & \\ O & 0.0000000 & 0.0000000 & 0.5754295 \\ F & -1.1037794 & 0.0000000 & -0.2877148 \\ F & 1.1037794 & 0.0000000 & -0.2877148 \\ & & & \\ & & & \\ 0 & -0.5017287 & 0.1821879 & 0.0000000 \\ \text { H } & -0.3643282 & -0.7782346 & 0.0000000 \\ F & 0.8660569 & 0.5960466 & 0.0000000\end{array}$


7. Optimized Structures at the PBEO Level

The Cartesian coordinates of the structures are listed in Ångström.

\begin{tabular}{|c|c|c|c|}
\hline C & 0.0000000 & -0.0000000 & -0.5611889 \\
\hline 0 & -0.0000000 & 0.0000000 & 0.5611889 \\
\hline $\mathrm{C}$ & 0.0000000 & 0.0000000 & 0.0931055 \\
\hline 0 & 0.0000000 & 0.0000000 & 1.2612583 \\
\hline $\mathrm{F}$ & 1.0578065 & 0.0000000 & -0.6771819 \\
\hline & -1.0578065 & 0.0000000 & -0.6771819 \\
\hline & -0.0000000 & -0.0000000 & 0.5911973 \\
\hline $\mathrm{C}$ & 0.0000000 & 0.0000000 & -0.5911974 \\
\hline$F$ & -0.0000000 & 0.0000000 & 1.8676921 \\
\hline$F$ & 0.0000000 & 0.0000000 & -1.8676920 \\
\hline C & 0.0000000 & -0.0000000 & 0.6473689 \\
\hline $\mathrm{C}$ & 0.0000000 & 0.0000000 & -0.5437339 \\
\hline $\mathrm{H}$ & -0.0000000 & -0.0000000 & 1.7090275 \\
\hline $\mathrm{F}$ & -0.0000000 & 0.0000000 & -1.8126625 \\
\hline $\mathrm{F}$ & 0.0000000 & -0.0000000 & -1.2212143 \\
\hline $\mathrm{C}$ & -0.0000000 & 0.0000000 & 0.0360818 \\
\hline $\mathrm{N}$ & 0.0000000 & -0.0000000 & 1.1851325 \\
\hline 0 & -0.9829797 & 0.3185388 & 0.0000000 \\
\hline $\mathrm{N}$ & 0.1292359 & 0.4899585 & 0.0000000 \\
\hline $\mathrm{F}$ & 0.8537438 & -0.8084974 & 0.0000000 \\
\hline $\mathrm{C}$ & 0.0000000 & 0.0000000 & -0.0057118 \\
\hline 0 & 0.0000000 & 0.0000000 & 1.1892378 \\
\hline $\mathrm{H}$ & 0.9395972 & 0.0000000 & -0.5917630 \\
\hline $\mathrm{H}$ & -0.9395972 & 0.0000000 & -0.5917630 \\
\hline 0 & 0.0000000 & 0.0000000 & 0.3891873 \\
\hline $\mathrm{H}$ & -0.7592269 & 0.0000000 & -0.1945942 \\
\hline $\mathrm{H}$ & 0.7592269 & -0.0000000 & -0.194594 \\
\hline $\mathrm{C}$ & -0.0000000 & 0.0000000 & 0.5979810 \\
\hline C & -0.0000000 & -0.0000000 & -0.5979806 \\
\hline $\mathrm{H}$ & 0.0000000 & 0.0000000 & 1.6618097 \\
\hline $\mathrm{H}$ & -0.0000000 & 0.0000000 & -1.661810 \\
\hline $\mathrm{H}$ & -0.0000000 & -0.0000000 & -1.0934004 \\
\hline $\mathrm{C}$ & 0.0000000 & 0.0000000 & -0.0256640 \\
\hline & -0.0000000 & -0.0000000 & 1.1190644 \\
\hline
\end{tabular}




$\begin{array}{rrrr}\text { C } & 0.0692499 & 0.0488252 & 0.0000000 \\ \text { O } & 1.2084045 & -0.2321360 & 0.0000000 \\ \text { F } & -0.9028435 & -0.8671801 & 0.0000000 \\ \text { H } & -0.3748109 & 1.0504910 & 0.0000000 \\ & & & \\ \text { O } & 0.0000000 & 0.0000000 & 0.5670860 \\ F & -1.0860129 & 0.0000000 & -0.2835430 \\ F & 1.0860129 & 0.0000000 & -0.2835430 \\ & & & \\ & & & \\ \text { O } & -0.4915430 & 0.1838280 & 0.0000000 \\ \text { H } & -0.3623929 & -0.7742108 & 0.0000000 \\ \text { F } & 0.8539358 & 0.5903828 & 0.0000000\end{array}$


8. Optimized Structures at the TPSSh Level

The Cartesian coordinates of the structures are listed in Ångström.

\begin{tabular}{|c|c|c|c|}
\hline C & 0.0000000 & -0.0000000 & -0.5642113 \\
\hline 0 & -0.0000000 & 0.0000000 & 0.5642113 \\
\hline C & 0.0000000 & 0.0000000 & 0.0968137 \\
\hline 0 & 0.0000000 & 0.0000000 & 1.2703223 \\
\hline F & 1.0654113 & 0.0000000 & -0.6835680 \\
\hline$F$ & -1.0654113 & 0.0000000 & -0.6835680 \\
\hline C & 0.0000000 & -0.0000000 & 0.5927139 \\
\hline C & 0.0000000 & -0.0000000 & -0.5927144 \\
\hline F & -0.0000000 & -0.0000000 & 1.8779012 \\
\hline $\mathrm{F}$ & -0.0000000 & 0.0000000 & -1.8779006 \\
\hline C & 0.0000000 & 0.0000000 & 0.6511661 \\
\hline C & -0.0000000 & 0.0000000 & -0.5426322 \\
\hline $\mathrm{H}$ & 0.0000000 & 0.0000000 & 1.7119418 \\
\hline $\mathrm{F}$ & -0.0000000 & 0.0000000 & -1.8204757 \\
\hline F & 0.0000000 & 0.0000000 & -1.2294808 \\
\hline C & -0.0000000 & 0.0000000 & 0.0372229 \\
\hline $\mathrm{N}$ & 0.0000000 & 0.0000000 & 1.1922579 \\
\hline 0 & -0.9949029 & 0.3263578 & 0.0000000 \\
\hline $\mathrm{N}$ & 0.1209353 & 0.5044807 & 0.0000000 \\
\hline $\mathrm{F}$ & 0.8739675 & -0.8308384 & 0.0000000 \\
\hline C & -0.0000000 & 0.0000000 & -0.0079247 \\
\hline 0 & 0.0000000 & 0.0000000 & 1.1942193 \\
\hline $\mathrm{H}$ & 0.9391364 & 0.0000000 & -0.5931473 \\
\hline $\mathrm{H}$ & -0.9391364 & 0.0000000 & -0.5931473 \\
\hline 0 & 0.0000000 & 0.0000000 & 0.3927688 \\
\hline $\mathrm{H}$ & -0.7619621 & 0.0000000 & -0.1963850 \\
\hline $\mathrm{H}$ & 0.7619621 & 0.0000000 & -0.1963850 \\
\hline $\mathrm{C}$ & -0.0000000 & -0.0000000 & 0.5990910 \\
\hline C & -0.0000000 & 0.0000000 & -0.5990914 \\
\hline $\mathrm{H}$ & 0.0000000 & -0.0000000 & 1.6619420 \\
\hline $\mathrm{H}$ & -0.0000000 & 0.0000000 & -1.6619416 \\
\hline $\mathrm{H}$ & -0.0000000 & -0.0000000 & -1.0945232 \\
\hline $\mathrm{C}$ & 0.0000000 & -0.0000000 & -0.027387 \\
\hline $\mathrm{N}$ & 0.0000000 & 0.0000000 & 1.1219103 \\
\hline
\end{tabular}




$\begin{array}{lrrr}\text { C } & 0.0723644 & 0.0537296 & 0.0000000 \\ \text { O } & 1.2159116 & -0.2322336 & 0.0000000 \\ \text { F } & -0.9119642 & -0.8725182 & 0.0000000 \\ \text { H } & -0.3763118 & 1.0510221 & 0.0000000 \\ & & & \\ & & & \\ \text { O } & 0.0000000 & 0.0000000 & 0.5766643 \\ \text { F } & -1.1084865 & 0.0000000 & -0.2883322 \\ \text { F } & 1.1084865 & -0.0000000 & -0.2883322 \\ & & & \\ & & & \\ \text { O } & -0.5050219 & 0.1836391 & 0.0000000 \\ \text { H } & -0.3624466 & -0.7786478 & 0.0000000 \\ \text { F } & 0.8674685 & 0.5950087 & 0.0000000\end{array}$


9. Optimized Structures at the LC- $\omega P B E$ Level

The Cartesian coordinates of the structures are listed in Ångström.

\begin{tabular}{|c|c|c|c|}
\hline C & 0.0000000 & 0.0000000 & -0.5604209 \\
\hline 0 & -0.0000000 & 0.0000000 & 0.5604209 \\
\hline C & 0.0000000 & 0.0000000 & 0.0941683 \\
\hline 0 & 0.0000000 & 0.0000000 & 1.2608945 \\
\hline $\mathrm{F}$ & 1.0599198 & 0.0000000 & -0.6775314 \\
\hline $\mathrm{F}$ & -1.0599198 & 0.0000000 & -0.6775314 \\
\hline $\mathrm{C}$ & -0.0000000 & 0.0000000 & 0.5889087 \\
\hline $\mathrm{C}$ & -0.0000000 & -0.0000000 & -0.5889087 \\
\hline $\mathrm{F}$ & 0.0000000 & 0.0000000 & 1.8699967 \\
\hline F & -0.0000000 & -0.0000000 & -1.8699967 \\
\hline C & -0.0000000 & 0.0000000 & 0.6455156 \\
\hline $\mathrm{C}$ & -0.0000000 & 0.0000000 & -0.5400185 \\
\hline $\mathrm{H}$ & 0.0000000 & 0.0000000 & 1.7084236 \\
\hline $\mathrm{F}$ & 0.0000000 & -0.0000000 & -1.8139207 \\
\hline $\mathrm{F}$ & -0.0000000 & -0.0000000 & -1.2215520 \\
\hline $\mathrm{C}$ & 0.0000000 & -0.0000000 & 0.0390545 \\
\hline $\mathrm{N}$ & -0.0000000 & 0.0000000 & 1.1824974 \\
\hline 0 & -0.9783975 & 0.3136463 & 0.0000000 \\
\hline $\mathrm{N}$ & 0.1349732 & 0.4854543 & 0.0000000 \\
\hline $\mathrm{F}$ & 0.8434243 & -0.7991007 & 0.0000000 \\
\hline $\mathrm{C}$ & 0.0000000 & 0.0000000 & -0.0078173 \\
\hline 0 & -0.0000000 & 0.0000000 & 1.1868438 \\
\hline $\mathrm{H}$ & 0.9392048 & 0.0000000 & -0.5895133 \\
\hline $\mathrm{H}$ & -0.9392048 & 0.0000000 & -0.5895133 \\
\hline 0 & 0.0000000 & 0.0000000 & 0.3866617 \\
\hline $\mathrm{H}$ & -0.7624245 & 0.0000000 & -0.1933314 \\
\hline $\mathrm{H}$ & 0.7624245 & -0.0000000 & -0.1933314 \\
\hline $\mathrm{C}$ & 0.0000000 & 0.0000000 & 0.5948370 \\
\hline C & 0.0000000 & 0.0000000 & -0.594836 \\
\hline $\mathrm{H}$ & -0.0000000 & 0.0000000 & 1.6598510 \\
\hline $\mathrm{H}$ & -0.0000000 & 0.0000000 & -1.6598513 \\
\hline H & -0.0000000 & 0.0000000 & -1.0919774 \\
\hline $\mathrm{C}$ & & 0.0000000 & -0.023781 \\
\hline $\mathrm{N}$ & 0.0000000 & -0.0000000 & 1.115758 \\
\hline
\end{tabular}


C $\quad 0.0698251$

0.0504137

0.0000000

o 1.2071684

$-0.2326959$

0.0000000

F $\quad-0.9027087$

$-0.8690376$

0.0000000

$\mathrm{H} \quad-0.3742848$

1.0513199

0.0000000

$0 \quad 0.0000000$

0.0000000

0.5656778

F $\quad-1.0798653$

0.0000000

$-0.2828389$

F $\quad 1.0798653$

0.0000000

$-0.2828389$

$0 \quad-0.4872905$

0.1845036

0.0000000

$\mathrm{H} \quad-0.3633694$

$-0.7748810$

0.0000000

F $\quad 0.8506599$

0.5903774

0.0000000 
10. Optimized Structures at the $\omega B 97 X-D$ Level

The Cartesian coordinates of the structures are listed in Ångström.

\begin{tabular}{|c|c|c|c|}
\hline C & -0.0000000 & -0.0000000 & -0.5609103 \\
\hline 0 & 0.0000000 & 0.0000000 & 0.5609103 \\
\hline $\mathrm{C}$ & 0.0000000 & 0.0000000 & 0.0922228 \\
\hline 0 & 0.0000000 & 0.0000000 & 1.2598975 \\
\hline $\mathrm{F}$ & 1.0584045 & -0.0000000 & -0.6760602 \\
\hline$F$ & -1.0584045 & 0.0000000 & -0.6760602 \\
\hline C & 0.0000000 & 0.0000000 & 0.5902228 \\
\hline $\mathrm{C}$ & 0.0000000 & 0.0000000 & -0.5902228 \\
\hline$F$ & -0.0000000 & 0.0000000 & 1.8672666 \\
\hline$F$ & -0.0000000 & 0.0000000 & -1.8672666 \\
\hline C & 0.0000000 & 0.0000000 & 0.6464886 \\
\hline $\mathrm{C}$ & 0.0000000 & 0.0000000 & -0.5423075 \\
\hline $\mathrm{H}$ & -0.0000000 & 0.0000000 & 1.7072444 \\
\hline $\mathrm{F}$ & -0.0000000 & -0.0000000 & -1.8114256 \\
\hline $\mathrm{F}$ & 0.0000000 & 0.0000000 & -1.2199189 \\
\hline $\mathrm{C}$ & 0.0000000 & 0.0000000 & 0.0366719 \\
\hline $\mathrm{N}$ & 0.0000000 & 0.0000000 & 1.1832470 \\
\hline 0 & -0.9822837 & 0.3178384 & 0.0000000 \\
\hline $\mathrm{N}$ & 0.1294028 & 0.4908936 & 0.0000000 \\
\hline $\mathrm{F}$ & 0.8528809 & -0.8087320 & 0.0000000 \\
\hline $\mathrm{C}$ & 0.0000000 & 0.0000000 & -0.0073664 \\
\hline 0 & 0.0000000 & 0.0000000 & 1.1872824 \\
\hline $\mathrm{H}$ & 0.9384498 & 0.0000000 & -0.5899580 \\
\hline $\mathrm{H}$ & -0.9384498 & 0.0000000 & -0.5899580 \\
\hline 0 & 0.0000000 & 0.0000000 & 0.3875062 \\
\hline $\mathrm{H}$ & -0.7587927 & 0.0000000 & -0.1937537 \\
\hline $\mathrm{H}$ & 0.7587927 & 0.0000000 & -0.1937537 \\
\hline $\mathrm{C}$ & -0.0000000 & 0.0000000 & 0.5967127 \\
\hline C & -0.0000000 & 0.0000000 & -0.5967127 \\
\hline $\mathrm{H}$ & 0.0000000 & 0.0000000 & 1.659226 \\
\hline $\mathrm{H}$ & 0.0000000 & 0.0000000 & -1.6592269 \\
\hline $\mathrm{H}$ & 0.0000000 & 0.0000000 & -1.0913316 \\
\hline $\mathrm{C}$ & 0.0000000 & 0.0000000 & -0.0255721 \\
\hline & 0.0000000 & -0.0000000 & 1.1169037 \\
\hline
\end{tabular}




$\begin{array}{lrrr}\text { C } & 0.0682681 & 0.0494144 & 0.0000000 \\ \text { O } & 1.2069297 & -0.2314693 & 0.0000000 \\ \text { F } & -0.9021618 & -0.8677474 & 0.0000000 \\ \text { H } & -0.3730361 & 1.0498022 & 0.0000000 \\ & & & \\ & & & \\ 0 & 0.0000000 & 0.0000000 & 0.5667852 \\ \text { F } & -1.0862535 & 0.0000000 & -0.2833926 \\ \text { F } & 1.0862535 & 0.0000000 & -0.2833926 \\ & & & \\ & & & \\ 0 & -0.4911012 & 0.1826595 & 0.0000000 \\ \text { H } & -0.3626406 & -0.7733021 & 0.0000000 \\ \text { F } & 0.8537417 & 0.5906426 & 0.0000000\end{array}$


11. Optimized Structures at the CAM-B3LYP Level

The Cartesian coordinates of the structures are listed in Ångström.

\begin{tabular}{|c|c|c|c|}
\hline $\mathrm{C}$ & -0.0000000 & -0.0000000 & -0.5596997 \\
\hline 0 & 0.0000000 & 0.0000000 & 0.5596997 \\
\hline C & 0.0000000 & 0.0000000 & 0.0945958 \\
\hline 0 & 0.0000000 & 0.0000000 & 1.2602769 \\
\hline $\mathrm{F}$ & 1.0592323 & 0.0000000 & -0.6774364 \\
\hline $\mathrm{F}$ & -1.0592323 & -0.0000000 & -0.6774364 \\
\hline $\mathrm{C}$ & -0.0000000 & 0.0000000 & 0.5886598 \\
\hline $\mathrm{C}$ & -0.0000000 & 0.0000000 & -0.5886598 \\
\hline $\mathrm{F}$ & 0.0000000 & 0.0000000 & 1.8688280 \\
\hline $\mathrm{F}$ & 0.0000000 & 0.0000000 & -1.8688280 \\
\hline C & -0.0000000 & 0.0000000 & 0.6460835 \\
\hline $\mathrm{C}$ & -0.0000000 & 0.0000000 & -0.5395733 \\
\hline $\mathrm{H}$ & 0.0000000 & 0.0000000 & 1.7059741 \\
\hline$F$ & 0.0000000 & 0.0000000 & -1.8124843 \\
\hline F & 0.0000000 & 0.0000000 & -1.2211820 \\
\hline C & 0.0000000 & 0.0000000 & 0.0388446 \\
\hline $\mathrm{N}$ & 0.0000000 & 0.0000000 & 1.1823374 \\
\hline 0 & -0.9817900 & 0.3180093 & 0.0000000 \\
\hline $\mathrm{N}$ & 0.1274296 & 0.4932383 & 0.0000000 \\
\hline $\mathrm{F}$ & 0.8543604 & -0.8112477 & 0.0000000 \\
\hline C & 0.0000000 & 0.0000000 & -0.0075581 \\
\hline 0 & 0.0000000 & 0.0000000 & 1.1868449 \\
\hline $\mathrm{H}$ & 0.9376720 & 0.0000000 & -0.5896434 \\
\hline $\mathrm{H}$ & -0.9376720 & 0.0000000 & -0.5896434 \\
\hline 0 & 0.0000000 & 0.0000000 & 0.3868502 \\
\hline $\mathrm{H}$ & -0.7635115 & 0.0000000 & -0.1934257 \\
\hline $\mathrm{H}$ & 0.7635115 & 0.0000000 & -0.1934257 \\
\hline C & -0.0000000 & 0.0000000 & 0.5952193 \\
\hline C & 0.0000000 & -0.0000000 & -0.5952079 \\
\hline $\mathrm{H}$ & 0.0000000 & 0.0000000 & 1.6570142 \\
\hline $\mathrm{H}$ & -0.0000000 & 0.0000000 & -1.6570255 \\
\hline $\mathrm{H}$ & 0.0000000 & 0.0000000 & -1.0902614 \\
\hline $\mathrm{C}$ & -0.0000000 & 0.0000000 & -0.0247311 \\
\hline $\mathrm{N}$ & 0.0000000 & 0.0000000 & 1.1149925 \\
\hline
\end{tabular}




$\begin{array}{rrrr}\text { C } & 0.0703544 & 0.0510788 & 0.0000000 \\ \text { O } & 1.2067288 & -0.2319164 & 0.0000000 \\ \text { F } & -0.9033941 & -0.8687031 & 0.0000000 \\ \text { H } & -0.3736890 & 1.0495407 & 0.0000000 \\ & & & \\ & & & \\ O & 0.0000000 & 0.0000000 & 0.5706897 \\ F & -1.0878123 & 0.0000000 & -0.2853448 \\ F & 1.0878123 & 0.0000000 & -0.2853448 \\ & & & \\ & & & \\ 0 & -0.4934464 & 0.1832670 & 0.0000000 \\ \text { H } & -0.3638938 & -0.7761656 & 0.0000000 \\ F & 0.8573401 & 0.5928986 & 0.0000000\end{array}$


12. Optimized Structures at the CAM-QPT-00 Level

The Cartesian coordinates of the structures are listed in Ångström.

\begin{tabular}{|c|c|c|c|}
\hline $\mathrm{C}$ & 0.0000000 & 0.0000000 & -0.5531337 \\
\hline 0 & -0.0000000 & 0.0000000 & 0.5531337 \\
\hline C & 0.0000000 & 0.0000000 & 0.0894470 \\
\hline 0 & 0.0000000 & 0.0000000 & 1.2427273 \\
\hline $\mathrm{F}$ & 1.0432298 & 0.0000000 & -0.6660871 \\
\hline $\mathrm{F}$ & -1.0432298 & 0.0000000 & -0.6660871 \\
\hline C & 0.0000000 & 0.0000000 & 0.5820631 \\
\hline $\mathrm{C}$ & 0.0000000 & 0.0000000 & -0.5820634 \\
\hline $\mathrm{F}$ & -0.0000000 & 0.0000000 & 1.8467363 \\
\hline F & -0.0000000 & -0.0000000 & -1.8467360 \\
\hline C & -0.0000000 & -0.0000000 & 0.6381386 \\
\hline $\mathrm{C}$ & -0.0000000 & -0.0000000 & -0.5355268 \\
\hline $\mathrm{H}$ & 0.0000000 & 0.0000000 & 1.6905211 \\
\hline $\mathrm{F}$ & 0.0000000 & 0.0000000 & -1.7931329 \\
\hline $\mathrm{F}$ & -0.0000000 & 0.0000000 & -1.2053136 \\
\hline $\mathrm{C}$ & -0.0000000 & 0.0000000 & 0.0383003 \\
\hline $\mathrm{N}$ & -0.0000000 & -0.0000000 & 1.1670133 \\
\hline 0 & -0.9622795 & 0.3063939 & 0.0000000 \\
\hline $\mathrm{N}$ & 0.1401825 & 0.4694637 & 0.0000000 \\
\hline $\mathrm{F}$ & 0.8220970 & -0.7758576 & 0.0000000 \\
\hline C & 0.0000000 & 0.0000000 & -0.0071048 \\
\hline 0 & 0.0000000 & 0.0000000 & 1.1736152 \\
\hline $\mathrm{H}$ & 0.9289360 & 0.0000000 & -0.5832552 \\
\hline $\mathrm{H}$ & -0.9289360 & 0.0000000 & -0.5832552 \\
\hline 0 & 0.0000000 & 0.0000000 & 0.3791401 \\
\hline $\mathrm{H}$ & -0.7562886 & 0.0000000 & -0.1895706 \\
\hline $\mathrm{H}$ & 0.7562886 & 0.0000000 & -0.1895706 \\
\hline C & -0.0000000 & -0.0000000 & 0.5899093 \\
\hline C & 0.0000000 & -0.0000000 & -0.5899093 \\
\hline $\mathrm{H}$ & -0.0000000 & -0.0000000 & 1.6441282 \\
\hline $\mathrm{H}$ & -0.0000000 & 0.0000000 & -1.6441282 \\
\hline $\mathrm{H}$ & 0.0000000 & 0.0000000 & -1.0807571 \\
\hline C & & 0.0000000 & -0.0231560 \\
\hline $\mathrm{N}$ & 0.0000000 & 0.0000000 & 1.1039131 \\
\hline
\end{tabular}




$\begin{array}{rrrr}\text { C } & 0.0649967 & 0.0469104 & 0.0000000 \\ \text { O } & 1.1906313 & -0.2298244 & 0.0000000 \\ \text { F } & -0.8874160 & -0.8571639 & 0.0000000 \\ \text { H } & -0.3682120 & 1.0400779 & 0.0000000 \\ & & & \\ \text { O } & 0.0000000 & 0.0000000 & 0.5571214 \\ F & -1.0568366 & 0.0000000 & -0.2785607 \\ \text { F } & 1.0568366 & 0.0000000 & -0.2785607 \\ & & & \\ & & & \\ \text { O } & -0.4760355 & 0.1810299 & 0.0000000 \\ \text { H } & -0.3619687 & -0.7656790 & 0.0000000 \\ \text { F } & 0.8380043 & 0.5846491 & 0.0000000\end{array}$


13. Optimized Structures at the CAM-QPT-02 Level

The Cartesian coordinates of the structures are listed in Ångström.

\begin{tabular}{|c|c|c|c|}
\hline C & -0.0000000 & 0.0000000 & -0.5561941 \\
\hline 0 & 0.0000000 & 0.0000000 & 0.5561941 \\
\hline C & 0.0000000 & 0.0000000 & 0.0916516 \\
\hline 0 & 0.0000000 & 0.0000000 & 1.2509437 \\
\hline $\mathrm{F}$ & 1.0497303 & 0.0000000 & -0.6712976 \\
\hline $\mathrm{F}$ & -1.0497303 & -0.0000000 & -0.6712976 \\
\hline C & -0.0000000 & 0.0000000 & 0.5852645 \\
\hline C & -0.0000000 & 0.0000000 & -0.5852645 \\
\hline $\mathrm{F}$ & 0.0000000 & 0.0000000 & 1.8569981 \\
\hline $\mathrm{F}$ & 0.0000000 & -0.0000000 & -1.8569981 \\
\hline C & 0.0000000 & -0.0000000 & 0.6408756 \\
\hline C & 0.0000000 & -0.0000000 & -0.5381921 \\
\hline $\mathrm{H}$ & -0.0000000 & 0.0000000 & 1.7000854 \\
\hline $\mathrm{F}$ & -0.0000000 & 0.0000000 & -1.8027689 \\
\hline $\mathrm{F}$ & -0.0000000 & -0.0000000 & -1.2124746 \\
\hline C & 0.0000000 & -0.0000000 & 0.0385687 \\
\hline $\mathrm{N}$ & -0.0000000 & 0.0000000 & 1.1739059 \\
\hline 0 & -0.9694843 & 0.3093523 & 0.0000000 \\
\hline $\mathrm{N}$ & 0.1374482 & 0.4779722 & 0.0000000 \\
\hline $\mathrm{F}$ & 0.8320361 & -0.7873245 & 0.0000000 \\
\hline C & -0.0000000 & 0.0000000 & -0.0075918 \\
\hline 0 & 0.0000000 & 0.0000000 & 1.1797030 \\
\hline $\mathrm{H}$ & 0.9364140 & 0.0000000 & -0.5860556 \\
\hline $\mathrm{H}$ & -0.9364140 & -0.0000000 & -0.5860556 \\
\hline 0 & 0.0000000 & 0.0000000 & 0.3831074 \\
\hline $\mathrm{H}$ & -0.7628800 & 0.0000000 & -0.1915542 \\
\hline $\mathrm{H}$ & 0.7628800 & -0.0000000 & -0.1915542 \\
\hline $\mathrm{C}$ & -0.0000000 & 0.0000000 & 0.5920790 \\
\hline C & 0.0000000 & 0.0000000 & -0.5920682 \\
\hline $\mathrm{H}$ & -0.0000000 & 0.0000000 & 1.6532041 \\
\hline $\mathrm{H}$ & -0.0000000 & 0.0000000 & -1.6532149 \\
\hline $\mathrm{H}$ & 0.0000000 & 0.0000000 & -1.0873088 \\
\hline $\mathrm{C}$ & -0.0000000 & 0.0000000 & -0.0226184 \\
\hline $\mathrm{N}$ & 0.0000000 & 0.0000000 & 1.1099272 \\
\hline
\end{tabular}




$\begin{array}{rrrr}\text { C } & 0.0672497 & 0.0482518 & 0.0000000 \\ \text { O } & 1.1977552 & -0.2325689 & 0.0000000 \\ \text { F } & -0.8929767 & -0.8628699 & 0.0000000 \\ \text { H } & -0.3720282 & 1.0471870 & 0.0000000 \\ & & & \\ & & & \\ 0 & 0.0000000 & 0.0000000 & 0.5629110 \\ F & -1.0666126 & 0.0000000 & -0.2814555 \\ F & 1.0666126 & 0.0000000 & -0.2814555 \\ & & & \\ & & & \\ 0 & -0.4819063 & 0.1840247 & 0.0000000 \\ H & -0.3631240 & -0.7722800 & 0.0000000 \\ F & 0.8450302 & 0.5882554 & 0.0000000\end{array}$


14. Optimized Structures at the LHO7t-SVWN Level

The Cartesian coordinates of the structures are listed in Ångström.

\begin{tabular}{|c|c|c|c|}
\hline C & 0.0000000 & 0.0000000 & -0.5628466 \\
\hline 0 & -0.0000000 & 0.0000000 & 0.5628466 \\
\hline C & 0.0000000 & 0.0000000 & 0.0949511 \\
\hline 0 & 0.0000000 & 0.0000000 & 1.2665105 \\
\hline $\mathrm{F}$ & 1.0644088 & 0.0000000 & -0.6807308 \\
\hline $\mathrm{F}$ & -1.0644088 & 0.0000000 & -0.6807308 \\
\hline C & -0.0000000 & -0.0000000 & 0.5926770 \\
\hline C & -0.0000000 & -0.0000000 & -0.5926775 \\
\hline $\mathrm{F}$ & 0.0000000 & -0.0000000 & 1.8763672 \\
\hline $\mathrm{F}$ & 0.0000000 & 0.0000000 & -1.8763667 \\
\hline C & 0.0000000 & 0.0000000 & 0.6496518 \\
\hline C & -0.0000000 & -0.0000000 & -0.5439292 \\
\hline $\mathrm{H}$ & -0.0000000 & 0.0000000 & 1.7146210 \\
\hline $\mathrm{F}$ & -0.0000000 & 0.0000000 & -1.8203436 \\
\hline $\mathrm{F}$ & -0.0000000 & 0.0000000 & -1.2262544 \\
\hline C & 0.0000000 & 0.0000000 & 0.0373181 \\
\hline $\mathrm{N}$ & -0.0000000 & 0.0000000 & 1.1889363 \\
\hline 0 & -0.9889115 & 0.3224258 & 0.0000000 \\
\hline $\mathrm{N}$ & 0.1262977 & 0.4942365 & 0.0000000 \\
\hline $\mathrm{F}$ & 0.8626138 & -0.8166623 & 0.0000000 \\
\hline $\mathrm{C}$ & -0.0000000 & 0.0000000 & -0.0083654 \\
\hline 0 & 0.0000000 & 0.0000000 & 1.1926241 \\
\hline $\mathrm{H}$ & 0.9412791 & 0.0000000 & -0.5921294 \\
\hline $\mathrm{H}$ & -0.9412791 & 0.0000000 & -0.592129 \\
\hline 0 & 0.0000000 & 0.0000000 & 0.3856020 \\
\hline $\mathrm{H}$ & -0.7647285 & 0.0000000 & -0.1928016 \\
\hline $\mathrm{H}$ & 0.7647285 & -0.0000000 & -0.192801 \\
\hline C & 0.0000000 & -0.0000000 & 0.5989538 \\
\hline C & 0.0000000 & 0.0000000 & -0.5989531 \\
\hline $\mathrm{H}$ & -0.0000000 & -0.0000000 & 1.6661855 \\
\hline $\mathrm{H}$ & 0.0000000 & 0.0000000 & -1.6661862 \\
\hline $\mathrm{H}$ & 0.0000000 & 0.0000000 & -1.0963131 \\
\hline $\mathrm{C}$ & -0.0000000 & 0.0000000 & -0.0252044 \\
\hline & 0.0000000 & 0.0000000 & 1.121517 \\
\hline
\end{tabular}




$\begin{array}{rrrr}\text { C } & 0.0709097 & 0.0512767 & 0.0000000 \\ \text { O } & 1.2133550 & -0.2320670 & 0.0000000 \\ \text { F } & -0.9091110 & -0.8725863 & 0.0000000 \\ \text { H } & -0.3751536 & 1.0533766 & 0.0000000 \\ & & & \\ \text { O } & 0.0000000 & 0.0000000 & 0.5689037 \\ \text { F } & -1.0957838 & 0.0000000 & -0.2844518 \\ \text { F } & 1.0957838 & 0.0000000 & -0.2844518 \\ & & & \\ & & & \\ \text { O } & -0.4950191 & 0.1827911 & 0.0000000 \\ \text { H } & -0.3646978 & -0.7769412 & 0.0000000 \\ \text { F } & 0.8597168 & 0.5941501 & 0.0000000\end{array}$


15. Optimized Structures at the LH12ct-SsirPW92 Level

The Cartesian coordinates of the structures are listed in Ångström.

\begin{tabular}{|c|c|c|c|}
\hline C & 0.0000000 & -0.0000000 & -0.5627007 \\
\hline 0 & -0.0000000 & 0.0000000 & 0.5627007 \\
\hline C & 0.0000000 & 0.0000000 & 0.0942465 \\
\hline 0 & 0.0000000 & 0.0000000 & 1.2655842 \\
\hline $\mathrm{F}$ & 1.0642541 & 0.0000000 & -0.6799154 \\
\hline $\mathrm{F}$ & -1.0642541 & 0.0000000 & -0.6799154 \\
\hline $\mathrm{C}$ & 0.0000000 & 0.0000000 & 0.5926234 \\
\hline $\mathrm{C}$ & 0.0000000 & 0.0000000 & -0.5926236 \\
\hline $\mathrm{F}$ & -0.0000000 & -0.0000000 & 1.8766961 \\
\hline$F$ & 0.0000000 & 0.0000000 & -1.8766959 \\
\hline C & -0.0000000 & 0.0000000 & 0.6497751 \\
\hline $\mathrm{C}$ & -0.0000000 & -0.0000000 & -0.5440943 \\
\hline $\mathrm{H}$ & 0.0000000 & -0.0000000 & 1.7151979 \\
\hline F & 0.0000000 & 0.0000000 & -1.8208787 \\
\hline $\mathrm{F}$ & 0.0000000 & -0.0000000 & -1.2259407 \\
\hline $\mathrm{C}$ & -0.0000000 & 0.0000000 & 0.0375146 \\
\hline $\mathrm{N}$ & 0.0000000 & 0.0000000 & 1.1884261 \\
\hline 0 & -0.9864049 & 0.3204952 & 0.0000000 \\
\hline $\mathrm{N}$ & 0.1294985 & 0.4890779 & 0.0000000 \\
\hline $\mathrm{F}$ & 0.8569064 & -0.8095731 & 0.0000000 \\
\hline $\mathrm{C}$ & -0.0000000 & 0.0000000 & -0.0088261 \\
\hline 0 & 0.0000000 & 0.0000000 & 1.1921611 \\
\hline $\mathrm{H}$ & 0.9408028 & 0.0000000 & -0.5916675 \\
\hline $\mathrm{H}$ & -0.9408028 & 0.0000000 & -0.591667 \\
\hline 0 & 0.0000000 & 0.0000000 & 0.3835191 \\
\hline $\mathrm{H}$ & -0.7635160 & 0.0000000 & -0.1917601 \\
\hline $\mathrm{H}$ & 0.7635160 & 0.0000000 & -0.1917601 \\
\hline $\mathrm{C}$ & 0.0000000 & 0.0000000 & 0.5992709 \\
\hline $\mathrm{C}$ & 0.0000000 & -0.0000000 & -0.5992703 \\
\hline $\mathrm{H}$ & -0.0000000 & 0.0000000 & 1.6670783 \\
\hline $\mathrm{H}$ & 0.0000000 & 0.0000000 & -1.6670789 \\
\hline $\mathrm{H}$ & -0.0000000 & 0.0000000 & -1.0965314 \\
\hline $\mathrm{C}$ & 0.0000000 & 0.0000000 & -0.024997 \\
\hline $\mathrm{N}$ & -0.0000000 & 0.0000000 & 1.1215291 \\
\hline
\end{tabular}




$\begin{array}{rrrr}\text { C } & 0.0701817 & 0.0505816 & 0.0000000 \\ \text { O } & 1.2128146 & -0.2314402 & 0.0000000 \\ \text { F } & -0.9086362 & -0.8722494 & 0.0000000 \\ \text { H } & -0.3743600 & 1.0531079 & 0.0000000 \\ & & & \\ \text { O } & 0.0000000 & 0.0000000 & 0.5657519 \\ \text { F } & -1.0905397 & 0.0000000 & -0.2828759 \\ \text { F } & 1.0905397 & -0.0000000 & -0.2828759 \\ & & & \\ & & & \\ \text { O } & -0.4912053 & 0.1819060 & 0.0000000 \\ \text { H } & -0.3650048 & -0.7751278 & 0.0000000 \\ \text { F } & 0.8562101 & 0.5932218 & 0.0000000\end{array}$


16. Optimized Structures at the LH14t-calPBE Level

The Cartesian coordinates of the structures are listed in Ångström.

\begin{tabular}{|c|c|c|c|}
\hline $\mathrm{C}$ & -0.0000000 & 0.0000000 & -0.5621448 \\
\hline 0 & 0.0000000 & 0.0000000 & 0.5621448 \\
\hline C & 0.0000000 & 0.0000000 & 0.0948830 \\
\hline 0 & 0.0000000 & 0.0000000 & 1.2650828 \\
\hline$F$ & 1.0629149 & 0.0000000 & -0.6799829 \\
\hline$F$ & -1.0629149 & 0.0000000 & -0.6799829 \\
\hline C & 0.0000000 & 0.0000000 & 0.5916332 \\
\hline C & 0.0000000 & 0.0000000 & -0.5916327 \\
\hline $\mathrm{F}$ & -0.0000000 & -0.0000000 & 1.8740535 \\
\hline$F$ & -0.0000000 & -0.0000000 & -1.8740540 \\
\hline C & 0.0000000 & 0.0000000 & 0.6489133 \\
\hline C & 0.0000000 & 0.0000000 & -0.5425228 \\
\hline $\mathrm{H}$ & -0.0000000 & -0.0000000 & 1.7114997 \\
\hline $\mathrm{F}$ & 0.0000000 & 0.0000000 & -1.8178902 \\
\hline $\mathrm{F}$ & -0.0000000 & 0.0000000 & -1.2250257 \\
\hline C & 0.0000000 & 0.0000000 & 0.0375741 \\
\hline $\mathrm{N}$ & 0.0000000 & 0.0000000 & 1.1874515 \\
\hline 0 & -0.9873763 & 0.3212157 & 0.0000000 \\
\hline $\mathrm{N}$ & 0.1273758 & 0.4933577 & 0.0000000 \\
\hline$F$ & 0.8600006 & -0.8145734 & 0.0000000 \\
\hline C & 0.0000000 & 0.0000000 & -0.0092891 \\
\hline 0 & 0.0000000 & 0.0000000 & 1.1907225 \\
\hline $\mathrm{H}$ & 0.9387746 & 0.0000000 & -0.5907167 \\
\hline $\mathrm{H}$ & -0.9387746 & 0.0000000 & -0.5907167 \\
\hline 0 & 0.0000000 & 0.0000000 & 0.3855838 \\
\hline $\mathrm{H}$ & -0.7641031 & 0.0000000 & -0.1927924 \\
\hline $\mathrm{H}$ & 0.7641031 & 0.0000000 & -0.1927924 \\
\hline C & 0.0000000 & 0.0000000 & 0.5979249 \\
\hline $\mathrm{C}$ & 0.0000000 & 0.0000000 & -0.5979241 \\
\hline $\mathrm{H}$ & -0.0000000 & -0.0000000 & 1.6626187 \\
\hline $\mathrm{H}$ & 0.0000000 & 0.0000000 & -1.6626195 \\
\hline $\mathrm{H}$ & 0.0000000 & 0.0000000 & -1.0940581 \\
\hline C & -0.0000000 & 0.0000000 & -0.0255371 \\
\hline $\mathrm{N}$ & 0.0000000 & 0.0000000 & 1.1195952 \\
\hline
\end{tabular}




$\begin{array}{rrrr}\text { C } & 0.0703487 & 0.0517969 & 0.0000000 \\ \text { O } & 1.2118241 & -0.2317942 & 0.0000000 \\ \text { F } & -0.9077357 & -0.8711085 & 0.0000000 \\ \text { H } & -0.3744371 & 1.0511058 & 0.0000000 \\ & & & \\ \text { O } & 0.0000000 & 0.0000000 & 0.5700140 \\ \text { F } & -1.0941535 & 0.0000000 & -0.2850070 \\ \text { F } & 1.0941535 & 0.0000000 & -0.2850070 \\ & & & \\ & & & \\ \text { O } & -0.4953212 & 0.1826959 & 0.0000000 \\ \text { H } & -0.3640389 & -0.7762255 & 0.0000000 \\ \text { F } & 0.8593601 & 0.5935296 & 0.0000000\end{array}$


17. Optimized Structures at the LH2Ot Level

The Cartesian coordinates of the structures are listed in Ångström.

\begin{tabular}{|c|c|c|c|}
\hline $\mathrm{C}$ & 0.0000000 & -0.0000000 & -0.5600895 \\
\hline 0 & -0.0000000 & 0.0000000 & 0.5600895 \\
\hline C & 0.0000000 & 0.0000000 & 0.0941284 \\
\hline 0 & 0.0000000 & 0.0000000 & 1.2600598 \\
\hline $\mathrm{F}$ & 1.0589511 & -0.0000000 & -0.6770941 \\
\hline $\mathrm{F}$ & -1.0589511 & 0.0000000 & -0.6770941 \\
\hline C & -0.0000000 & -0.0000000 & 0.5891458 \\
\hline C & -0.0000000 & 0.0000000 & -0.5891459 \\
\hline $\mathrm{F}$ & 0.0000000 & 0.0000000 & 1.8689974 \\
\hline F & -0.0000000 & 0.0000000 & -1.8689972 \\
\hline C & 0.0000000 & 0.0000000 & 0.6467961 \\
\hline $\mathrm{C}$ & -0.0000000 & -0.0000000 & -0.5399173 \\
\hline $\mathrm{H}$ & 0.0000000 & -0.0000000 & 1.7061088 \\
\hline$F$ & 0.0000000 & 0.0000000 & -1.8129875 \\
\hline $\mathrm{F}$ & -0.0000000 & 0.0000000 & -1.2213128 \\
\hline $\mathrm{C}$ & 0.0000000 & 0.0000000 & 0.0383614 \\
\hline $\mathrm{N}$ & -0.0000000 & 0.0000000 & 1.1829515 \\
\hline 0 & -0.9812727 & 0.3174130 & 0.0000000 \\
\hline $\mathrm{N}$ & 0.1307174 & 0.4873747 & 0.0000000 \\
\hline $\mathrm{F}$ & 0.8505553 & -0.8047877 & 0.0000000 \\
\hline $\mathrm{C}$ & -0.0000000 & 0.0000000 & -0.0109371 \\
\hline 0 & 0.0000000 & 0.0000000 & 1.1858334 \\
\hline $\mathrm{H}$ & 0.9341585 & 0.0000000 & -0.5874481 \\
\hline $\mathrm{H}$ & -0.9341585 & 0.0000000 & -0.5874481 \\
\hline 0 & 0.0000000 & 0.0000000 & 0.3818519 \\
\hline $\mathrm{H}$ & -0.7632764 & 0.0000000 & -0.1909265 \\
\hline $\mathrm{H}$ & 0.7632764 & 0.0000000 & -0.1909265 \\
\hline $\mathrm{C}$ & 0.0000000 & 0.0000000 & 0.5957162 \\
\hline C & 0.0000000 & 0.0000000 & -0.5957155 \\
\hline $\mathrm{H}$ & -0.0000000 & 0.0000000 & 1.6570781 \\
\hline $\mathrm{H}$ & 0.0000000 & -0.0000000 & -1.6570788 \\
\hline H & 0.0000000 & 0.0000000 & -1.090088 \\
\hline C & -0.0000000 & -0.0000000 & -0.0252064 \\
\hline $\mathrm{N}$ & -0.0000000 & 0.0000000 & 1.1152947 \\
\hline
\end{tabular}




$\begin{array}{rrrr}\text { C } & 0.0690560 & 0.0519251 & 0.0000000 \\ \text { O } & 1.2067384 & -0.2307653 & 0.0000000 \\ \text { F } & -0.9036963 & -0.8679989 & 0.0000000 \\ \text { H } & -0.3720981 & 1.0468390 & 0.0000000 \\ & & & \\ \text { O } & 0.0000000 & 0.0000000 & 0.5676389 \\ F & -1.0858793 & 0.0000000 & -0.2838194 \\ F & 1.0858793 & 0.0000000 & -0.2838194 \\ & & & \\ & & & \\ \text { O } & -0.4910393 & 0.1817017 & 0.0000000 \\ \text { H } & -0.3637728 & -0.7735177 & 0.0000000 \\ F & 0.8548120 & 0.5918161 & 0.0000000\end{array}$


18. Optimized Structures at the mPSTS Level

The Cartesian coordinates of the structures are listed in Ångström.

\begin{tabular}{|c|c|c|c|}
\hline C & -0.0000000 & 0.0000000 & -0.5645419 \\
\hline 0 & 0.0000000 & 0.0000000 & 0.5645419 \\
\hline C & 0.0000000 & 0.0000000 & 0.0965035 \\
\hline 0 & 0.0000000 & 0.0000000 & 1.2706047 \\
\hline F & 1.0661526 & 0.0000000 & -0.6835541 \\
\hline $\mathrm{F}$ & -1.0661526 & 0.0000000 & -0.6835541 \\
\hline C & -0.0000000 & -0.0000000 & 0.5928929 \\
\hline C & 0.0000000 & 0.0000000 & -0.5928936 \\
\hline $\mathrm{F}$ & -0.0000000 & -0.0000000 & 1.8790351 \\
\hline $\mathrm{F}$ & -0.0000000 & -0.0000000 & -1.8790344 \\
\hline C & 0.0000000 & 0.0000000 & 0.6516251 \\
\hline C & 0.0000000 & -0.0000000 & -0.5426988 \\
\hline $\mathrm{H}$ & -0.0000000 & 0.0000000 & 1.7125403 \\
\hline$F$ & -0.0000000 & 0.0000000 & -1.8214665 \\
\hline F & -0.0000000 & -0.0000000 & -1.2302013 \\
\hline C & 0.0000000 & 0.0000000 & 0.0373162 \\
\hline $\mathrm{N}$ & -0.0000000 & -0.0000000 & 1.1928850 \\
\hline 0 & -0.9951431 & 0.3261884 & 0.0000000 \\
\hline $\mathrm{N}$ & 0.1213292 & 0.5039424 & 0.0000000 \\
\hline $\mathrm{F}$ & 0.8738138 & -0.8301308 & 0.0000000 \\
\hline C & 0.0000000 & 0.0000000 & -0.0078476 \\
\hline 0 & 0.0000000 & 0.0000000 & 1.1946906 \\
\hline $\mathrm{H}$ & 0.9388627 & -0.0000000 & -0.5934215 \\
\hline $\mathrm{H}$ & -0.9388627 & 0.0000000 & -0.5934215 \\
\hline 0 & 0.0000000 & 0.0000000 & 0.3928161 \\
\hline $\mathrm{H}$ & -0.7609692 & 0.0000000 & -0.1964086 \\
\hline $\mathrm{H}$ & 0.7609692 & 0.0000000 & -0.1964086 \\
\hline $\mathrm{C}$ & 0.0000000 & 0.0000000 & 0.5994072 \\
\hline C & 0.0000000 & 0.0000000 & -0.5994082 \\
\hline $\mathrm{H}$ & -0.0000000 & 0.0000000 & 1.6624327 \\
\hline $\mathrm{H}$ & 0.0000000 & -0.0000000 & -1.6624317 \\
\hline $\mathrm{H}$ & -0.0000000 & 0.0000000 & -1.0947424 \\
\hline $\mathrm{C}$ & 0.0000000 & 0.0000000 & -0.0275745 \\
\hline $\mathrm{N}$ & 0.0000000 & 0.0000000 & 1.1223169 \\
\hline
\end{tabular}




$\begin{array}{lrrr}\text { C } & 0.0721330 & 0.0536384 & 0.0000000 \\ \text { O } & 1.2164363 & -0.2318089 & 0.0000000 \\ \text { F } & -0.9125869 & -0.8728035 & 0.0000000 \\ \text { H } & -0.3759824 & 1.0509740 & 0.0000000 \\ & & & \\ & & & \\ \text { O } & 0.0000000 & 0.0000000 & 0.5756949 \\ F & -1.1083059 & 0.0000000 & -0.2878474 \\ F & 1.1083059 & 0.0000000 & -0.2878474 \\ & & & \\ & & & \\ \text { O } & -0.5043017 & 0.1831706 & 0.0000000 \\ \text { H } & -0.3625573 & -0.7781983 & 0.0000000 \\ F & 0.8668590 & 0.5950277 & 0.0000000\end{array}$




\section{Optimized Structures at the LHJ14 Level}

The Cartesian coordinates of the structures are listed in Ångström.

\begin{tabular}{|c|c|c|c|}
\hline $\mathrm{C}$ & -0.0000000 & 0.0000000 & -0.5619822 \\
\hline 0 & 0.0000000 & 0.0000000 & 0.5619822 \\
\hline C & 0.0000000 & 0.0000000 & 0.0962429 \\
\hline 0 & 0.0000000 & 0.0000000 & 1.2656576 \\
\hline$F$ & 1.0616130 & 0.0000000 & -0.6809503 \\
\hline$F$ & -1.0616130 & -0.0000000 & -0.6809503 \\
\hline C & -0.0000000 & 0.0000000 & 0.5907282 \\
\hline C & 0.0000000 & -0.0000000 & -0.5907266 \\
\hline $\mathrm{F}$ & -0.0000000 & 0.0000000 & 1.8715756 \\
\hline $\mathrm{F}$ & -0.0000000 & 0.0000000 & -1.8715772 \\
\hline C & -0.0000000 & 0.0000000 & 0.6485363 \\
\hline C & 0.0000000 & -0.0000000 & -0.5402926 \\
\hline $\mathrm{H}$ & 0.0000000 & -0.0000000 & 1.7058933 \\
\hline$F$ & -0.0000000 & 0.0000000 & -1.8141370 \\
\hline$F$ & 0.0000000 & -0.0000000 & -1.2243071 \\
\hline C & -0.0000000 & 0.0000000 & 0.0377755 \\
\hline $\mathrm{N}$ & 0.0000000 & 0.0000000 & 1.1865316 \\
\hline 0 & -0.9887227 & 0.3223100 & 0.0000000 \\
\hline $\mathrm{N}$ & 0.1236308 & 0.5008772 & 0.0000000 \\
\hline $\mathrm{F}$ & 0.8650919 & -0.8231872 & 0.0000000 \\
\hline C & -0.0000000 & 0.0000000 & -0.0096146 \\
\hline 0 & 0.0000000 & 0.0000000 & 1.1885439 \\
\hline $\mathrm{H}$ & 0.9359207 & 0.0000000 & -0.5894647 \\
\hline $\mathrm{H}$ & -0.9359207 & 0.0000000 & -0.5894647 \\
\hline 0 & 0.0000000 & 0.0000000 & 0.3885155 \\
\hline $\mathrm{H}$ & -0.7627429 & 0.0000000 & -0.1942583 \\
\hline $\mathrm{H}$ & 0.7627429 & 0.0000000 & -0.1942583 \\
\hline C & 0.0000000 & 0.0000000 & 0.5962715 \\
\hline $\mathrm{C}$ & 0.0000000 & -0.0000000 & -0.5962719 \\
\hline $\mathrm{H}$ & 0.0000000 & -0.0000000 & 1.6555328 \\
\hline $\mathrm{H}$ & 0.0000000 & 0.0000000 & -1.6555324 \\
\hline $\mathrm{H}$ & 0.0000000 & 0.0000000 & -1.0898652 \\
\hline C & -0.0000000 & 0.0000000 & -0.0267214 \\
\hline $\mathrm{N}$ & 0.0000000 & -0.0000000 & 1.1165867 \\
\hline
\end{tabular}




$\begin{array}{rrrr}\text { C } & 0.0715878 & 0.0536324 & 0.0000000 \\ \text { O } & 1.2108231 & -0.2320227 & 0.0000000 \\ \text { F } & -0.9074982 & -0.8695873 & 0.0000000 \\ \text { H } & -0.3749127 & 1.0479775 & 0.0000000 \\ & & & \\ \text { O } & 0.0000000 & 0.0000000 & 0.5761721 \\ \text { F } & -1.0984542 & 0.0000000 & -0.2880860 \\ \text { F } & 1.0984542 & 0.0000000 & -0.2880860 \\ & & & \\ & & & \\ \text { O } & -0.5015578 & 0.1836372 & 0.0000000 \\ \text { H } & -0.3612519 & -0.7761915 & 0.0000000 \\ \text { F } & 0.8628097 & 0.5925542 & 0.0000000\end{array}$




\section{S5. NMR SHIELDING DATA}

\section{A. NMR Shieldings and Shifts of the ${ }^{1} \mathrm{H}$ and ${ }^{13} \mathrm{C}$ Test Set}

This subsection lists the individual NMR isotropic shielding constants of the test set by the Ochsenfeld group [67]. Structures were taken from Ref. 67. Tight thresholds were applied for the self-consistent field (SCF) procedure; energies were converged up to $10^{-9} \mathrm{E}_{\mathrm{h}}$ and a threshold of $10^{-9}$ a.u. was applied for the root mean square of the density matrix. Furthermore, the norm of the residuum in the coupled-perturbed Kohn-Sham (CPKS) equations [6] was required to be less than $10^{-7}$ a.u. to ensure a well converged perturbed density matrix. The def2-TZVP basis set [15] was employed throughout. The following density functionals are considered: KT3 [68, 69], BP86 [34, 35], PBE [36], TPSS [70], BH\&HLYP [34, 71, 72], B3LYP [71, 73, 74], PBE0 [75], TPSSh [76], LC- $\omega$ PBE [77--79], $\omega$ B97X-D [78, 80], CAM-B3LYP [78, 79, 81], CAM-QTP-00[78, 79, 82], CAM-QTP02[78, 79, 83], LH07t-SVWN [84], LH12ct-SsirPW92 [85], LH14t-calPBE [86], LH20t [87], Johnson's LHJ14 [88], and the mPSTS functional developed herein. Large grids (gridsize 4) are used for the numerical integration of the exchange correlation terms [7]. The seminumerical exchange approach [89-91] is only applied to the local hybrid functionals (LHFs). All calculations in this work were carried out with TURBOMOLE [10-13, 43, 45] Both ${ }^{1} \mathrm{H}$ and ${ }^{13} \mathrm{C}$ NMR shifts are measured with respect to tetramethylsilane (TMS). All NMR data in this section is given in ppm. The def2-TZVP/CCSD(T) results serve as reference [67].

TABLE S32. Statistical evaluation of ${ }^{1} \mathrm{H}$ NMR shifts in ppm of various computational methods with respect to CCSD(T). LH07t, LH12ct, and LH14t refer to the functionals LH07t-SVWN, LH12ct-SsirPW92, and LH14t-calPBE, respectively. MAE, MSD, and STD denote the mean absolute error, mean signed error, and the standard deviation regarding the latter.

\begin{tabular}{|c|c|c|c|c|c|c|c|c|c|c|c|c|c|c|c|c|c|c|}
\hline & $\mathrm{HF}$ & KT3 & BP86 & PBE & TPSS & BH\&HLYP & B3LYP & PBE0 & TPSSh & LC- $\omega$ PBE & $\omega \mathrm{B} 97 \mathrm{X}-\mathrm{D}$ & CAM-B3LYP & LH07t & LH12ct & LH14t & LH20t & mPSTS & LHJ14 \\
\hline MAE & 0.17 & 0.14 & 0.20 & 0.22 & 0.15 & 0.14 & 0.18 & 0.17 & 0.14 & 0.17 & 0.16 & 0.17 & 0.13 & 0.15 & 0.11 & 0.11 & 0.11 & 0.18 \\
\hline Max. Deviation & 0.47 & 0.61 & 1.05 & 1.11 & 0.67 & 0.42 & 0.77 & 0.76 & 0.57 & 0.61 & 0.68 & 0.70 & 0.41 & 0.48 & 0.37 & 0.41 & 0.52 & 0.85 \\
\hline MSD & 0.11 & 0.07 & 0.13 & 0.13 & 0.10 & 0.13 & 0.15 & 0.13 & 0.11 & 0.13 & 0.13 & 0.15 & 0.10 & 0.09 & 0.08 & 0.07 & 0.08 & 0.11 \\
\hline STD & 0.17 & 0.19 & 0.26 & 0.28 & 0.18 & 0.10 & 0.17 & 0.18 & 0.16 & 0.19 & 0.16 & 0.15 & 0.13 & 0.16 & 0.11 & 0.12 & 0.14 & 0.22 \\
\hline
\end{tabular}

TABLE S33. Statistical evaluation ${ }^{13} \mathrm{C}$ NMR shifts (in ppm) of various computational methods with respect to CCSD(T). LH07t, LH12ct, and LH14t refer to the functionals LH07t-SVWN, LH12ct-SsirPW92, and LH14t-calPBE, respectively. MAE, MSD, and STD denote the mean absolute error, mean signed error, and the standard deviation regarding the latter.

\begin{tabular}{|c|c|c|c|c|c|c|c|c|c|c|c|c|c|c|c|c|c|c|}
\hline & $\mathrm{HF}$ & KT3 & BP86 & PBE & TPSS & BH\&HLYP & B3LYP & PBE0 & TPSSh & LC- $\omega$ PBE & $\omega \mathrm{B} 97 \mathrm{X}-\mathrm{D}$ & CAM-B3LYP & LH07t & LH12ct & LH14t & LH20t & mPSTS & LHJ14 \\
\hline MAE & 8.6 & 4.5 & 4.4 & 4.5 & 2.4 & 7.0 & 6.0 & 6.1 & 2.8 & 9.1 & 6.4 & 8.0 & 7.7 & 8.4 & 6.2 & 6.9 & 2.3 & 7.4 \\
\hline Max. Deviation & 31.7 & 14.6 & 22.6 & 21.8 & 18.7 & 21.4 & 19.7 & 17.5 & 15.9 & 23.2 & 17.1 & 24.9 & 19.1 & 19.3 & 17.7 & 19.2 & 14.3 & 21.4 \\
\hline MSD & 6.8 & -2.6 & 3.9 & 4.2 & 1.8 & 6.9 & 5.4 & 5.8 & 2.6 & 8.9 & 6.3 & 7.8 & 7.5 & 8.2 & 6.1 & 6.9 & 2.1 & 6.9 \\
\hline STD & 8.6 & 4.8 & 4.9 & 4.9 & 3.7 & 5.9 & 5.3 & 5.0 & 3.2 & 6.6 & 4.8 & 6.3 & 5.4 & 5.8 & 4.9 & 5.4 & 2.9 & 6.3 \\
\hline
\end{tabular}




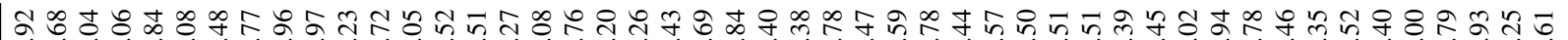

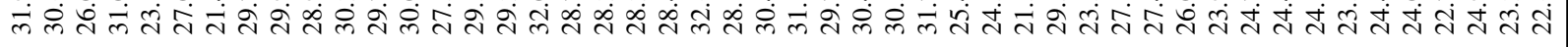

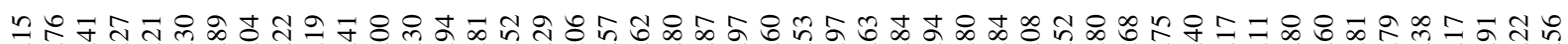

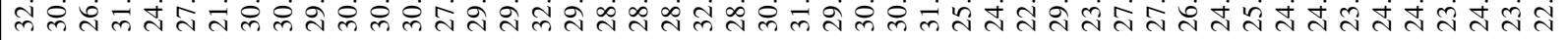

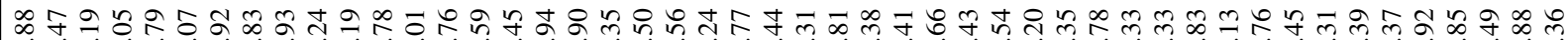

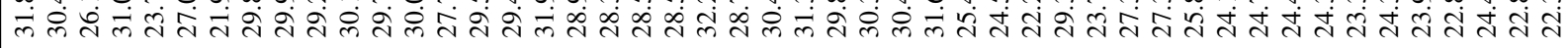
б 年品

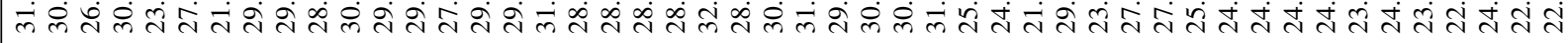

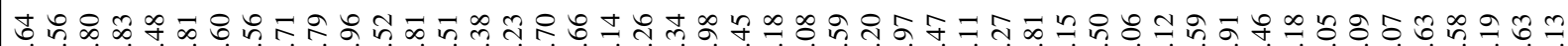

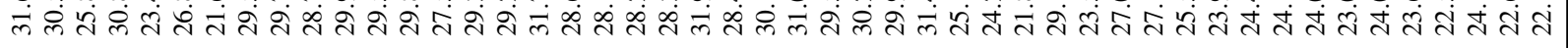
의영

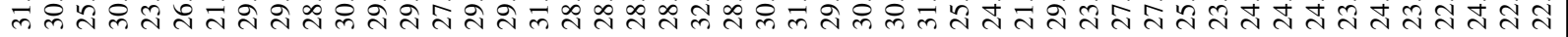

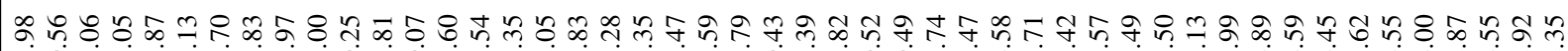

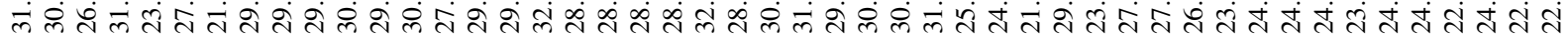

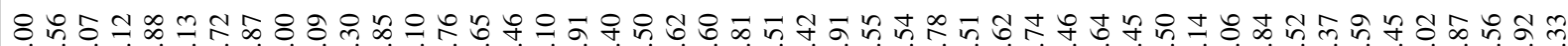

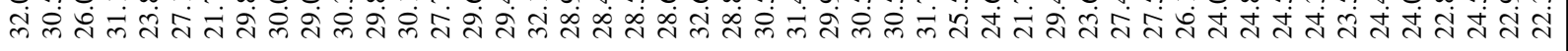

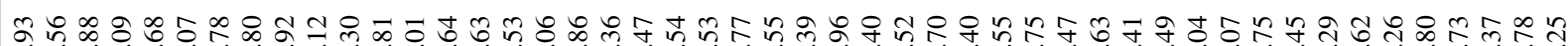

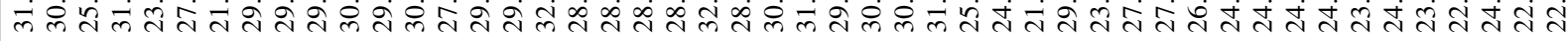

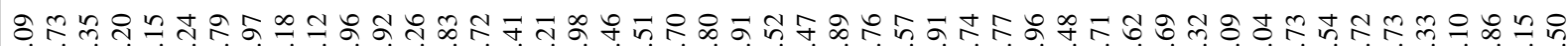

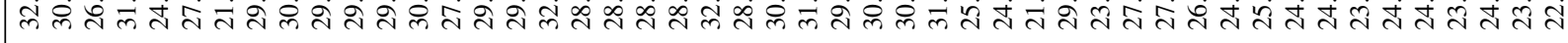

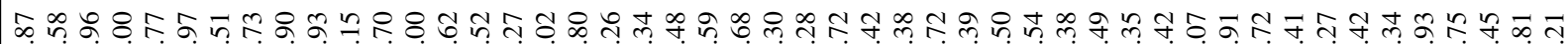

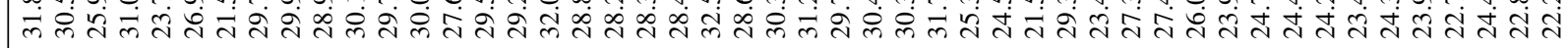

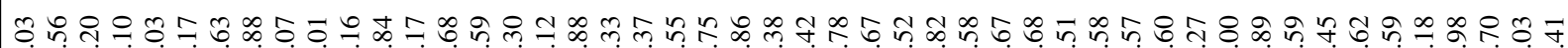

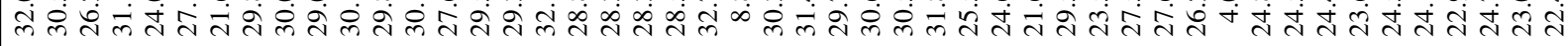

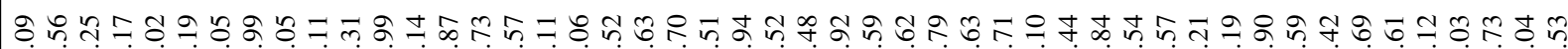

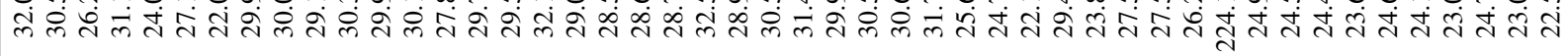

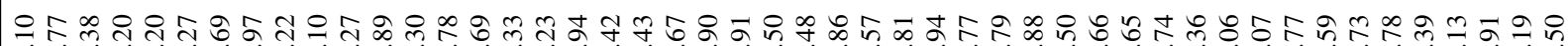

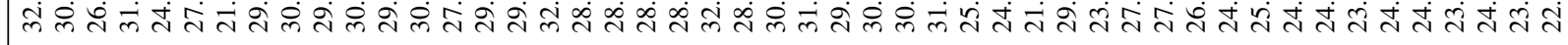
চ

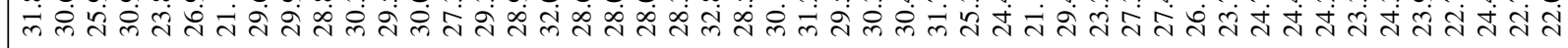

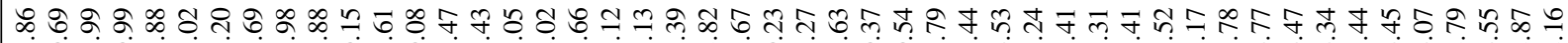

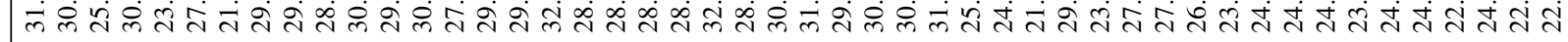

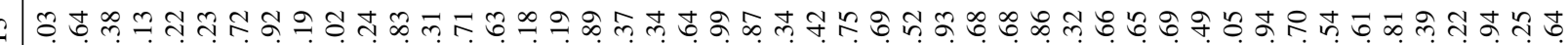

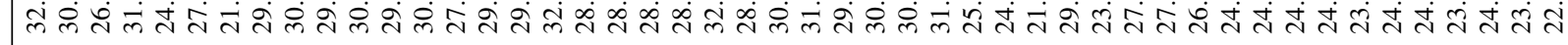

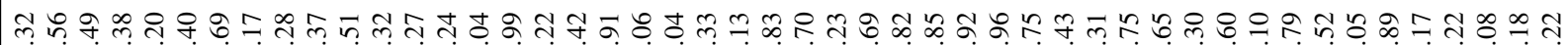

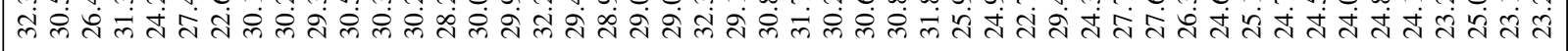




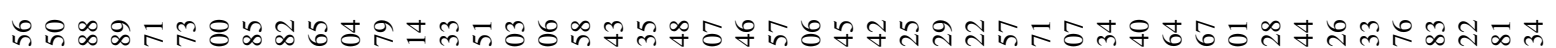

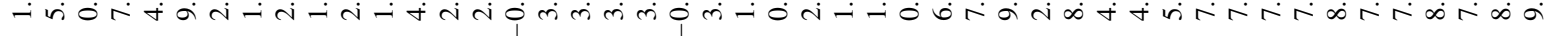

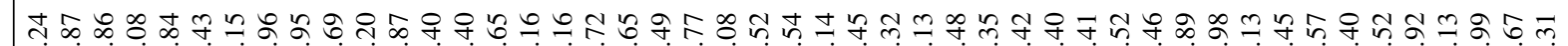

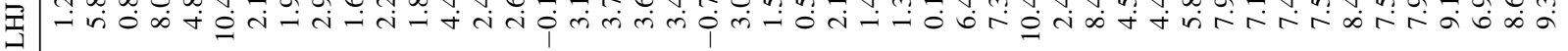

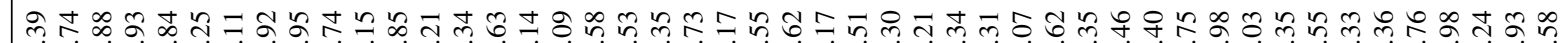

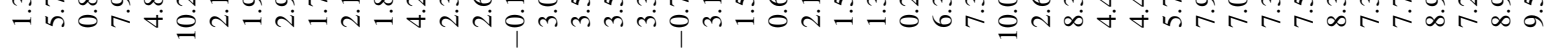
F

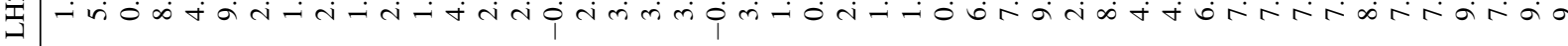

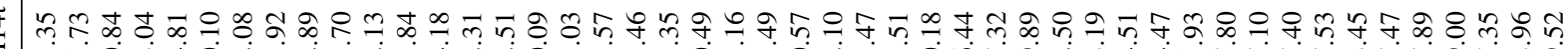

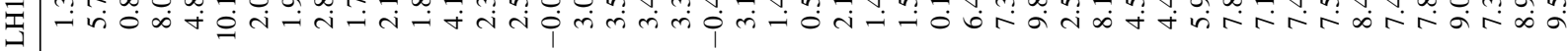
乐英 I -

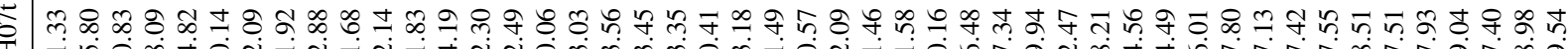

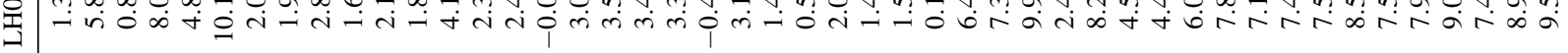

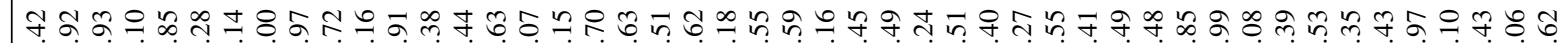

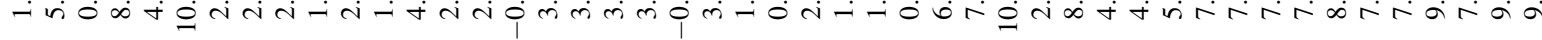

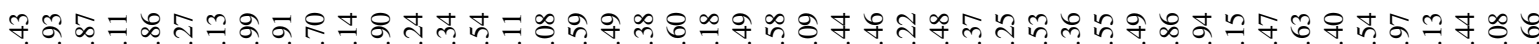

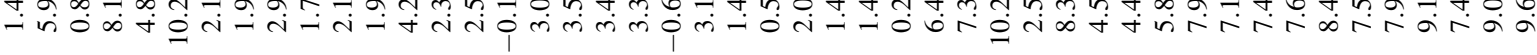

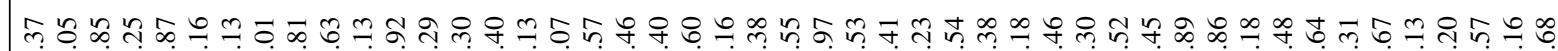
- 0 ó

퐁

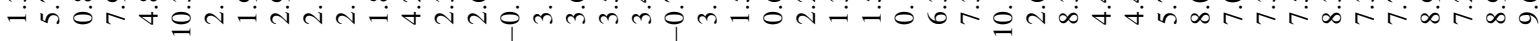

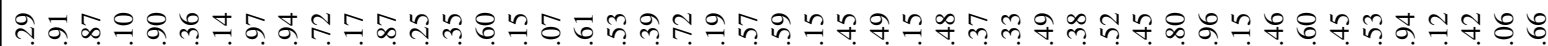

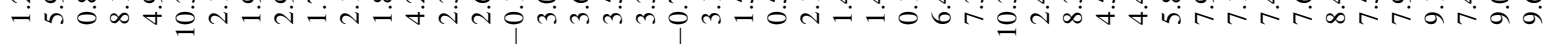

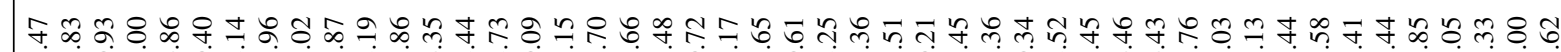

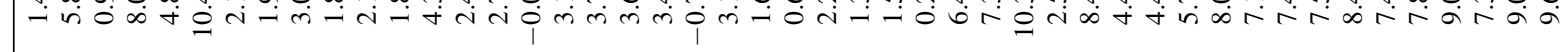

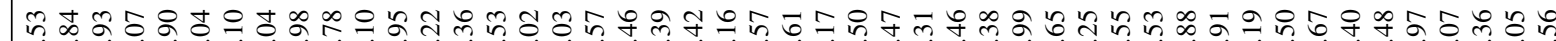

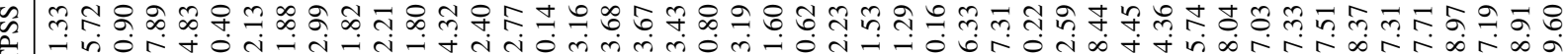

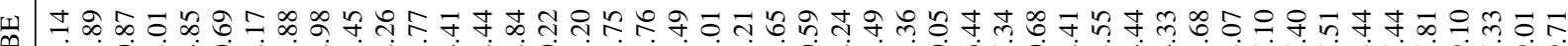

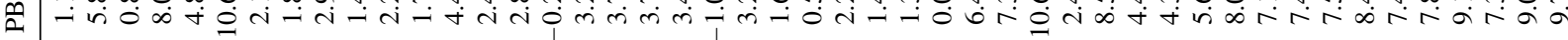

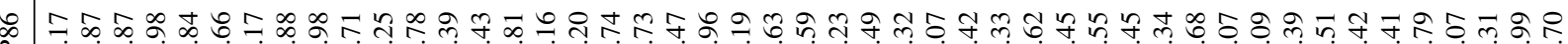

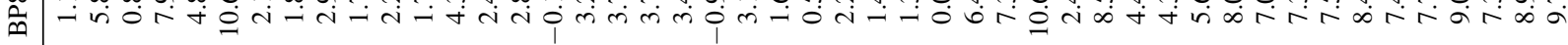
๓

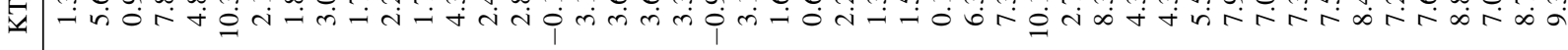

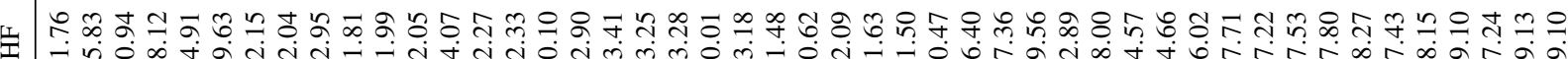

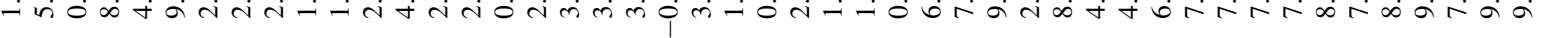

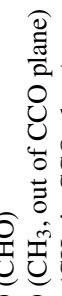

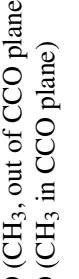

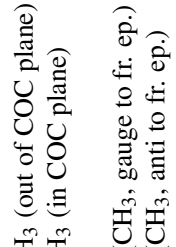

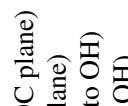
8 영 낭

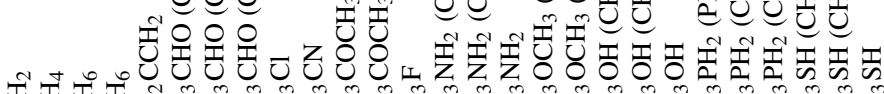
迅焉

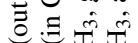

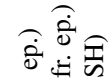
$\dot{1} 0$ 온 $\therefore$ 品品

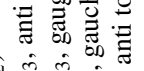

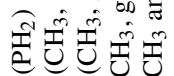

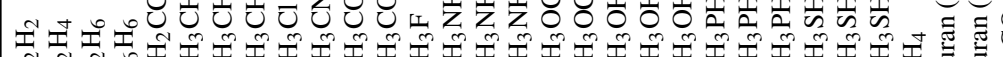
Æृ̂̃

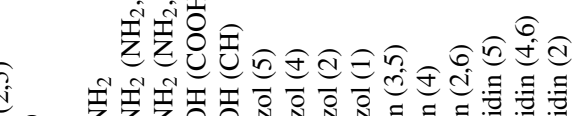




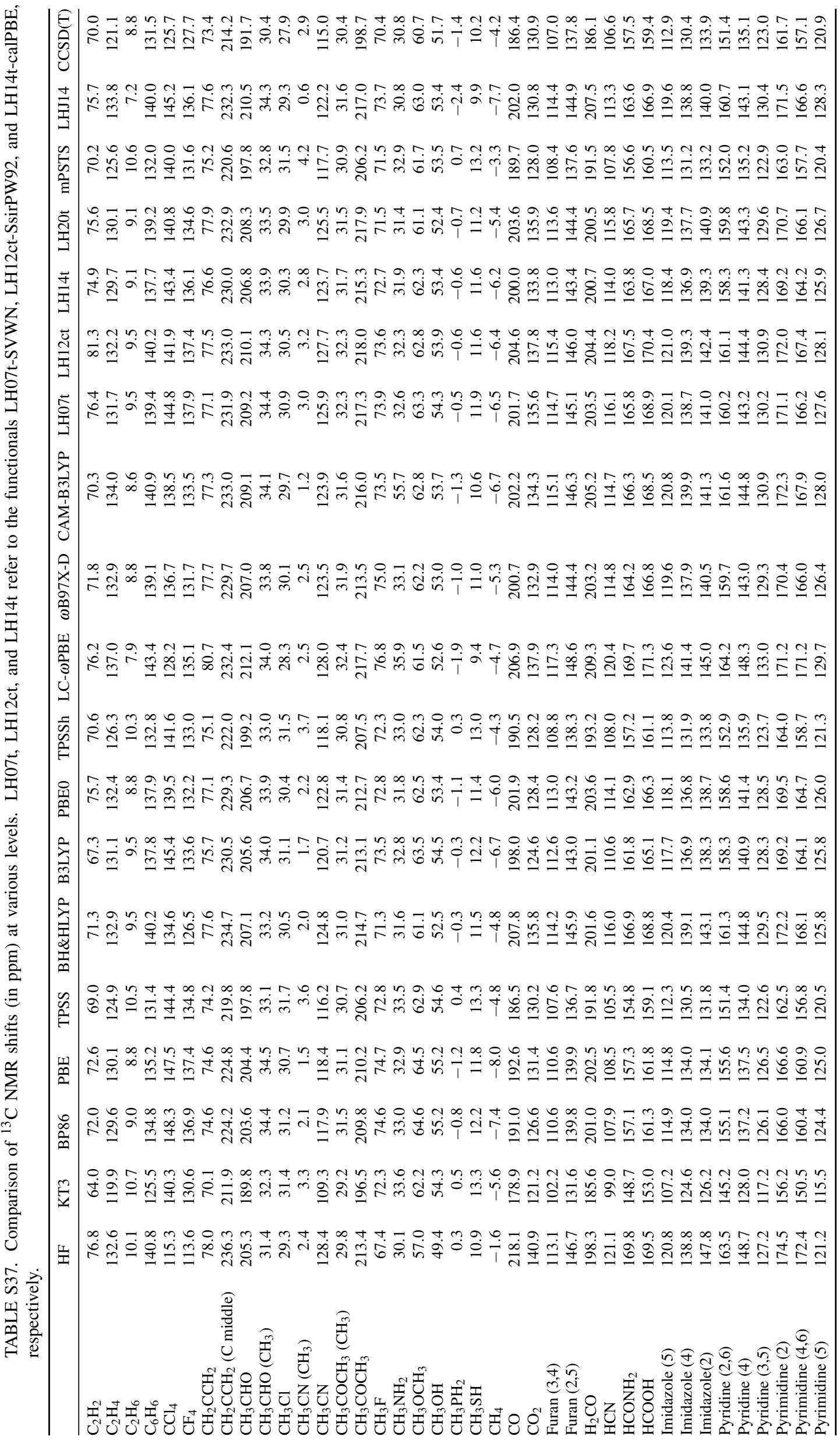


TABLE S38. Statistical evaluation of ${ }^{1} \mathrm{H}$ NMR shifts in ppm of various functionals including the current density response with respect to CCSD(T). LH07t, LH12ct, and LH14t refer to the functionals LH07t-SVWN, LH12ct-SsirPW92, and LH14t-calPBE, respectively. MAE, MSD, and STD denote the mean absolute error, mean signed error, and the standard deviation regarding the latter.

\begin{tabular}{lcccccccccc}
\hline & CAM-QTP-00 & CAM-QTP-02 & cTPSS & cTPSSh & cLH07t & cLH12ct & cLH14t & cLH20t & cmPSTS & cLHJ14 \\
\hline MAE & 0.15 & 0.19 & 0.14 & 0.13 & 0.13 & 0.15 & 0.10 & 0.10 & 0.13 & 0.15 \\
Max. Deviation & 0.40 & 0.60 & 0.65 & 0.57 & 0.41 & 0.46 & 0.33 & 0.39 & 0.95 & 0.68 \\
MSD & 0.14 & 0.17 & 0.10 & 0.10 & 0.10 & 0.11 & 0.08 & 0.06 & 0.10 & 0.09 \\
STD & 0.12 & 0.16 & 0.17 & 0.14 & 0.13 & 0.16 & 0.11 & 0.11 & 0.18 & 0.18 \\
\hline
\end{tabular}

TABLE S39. Statistical evaluation of ${ }^{13} \mathrm{C}$ NMR shifts in ppm of various functionals including the current density response with respect to CCSD(T). LH07t, LH12ct, and LH14t refer to the functionals LH07t-SVWN, LH12ct-SsirPW92, and LH14t-calPBE, respectively. MAE, MSD, and STD denote the mean absolute error, mean signed error, and the standard deviation regarding the latter.

\begin{tabular}{|c|c|c|c|c|c|c|c|c|c|c|}
\hline & CAM-QTP-00 & CAM-QTP-02 & cTPSS & cTPSSh & $\mathrm{cLH} 07 \mathrm{t}$ & $\mathrm{cLH} 12 \mathrm{ct}$ & $\mathrm{cLH} 14 \mathrm{t}$ & cLH20t & cmPSTS & cLHJ14 \\
\hline MAE & 8.5 & 9.5 & 2.8 & 3.2 & 6.0 & 6.2 & 5.6 & 6.0 & 2.9 & 6.0 \\
\hline Max. Deviation & 26.1 & 23.6 & 19.0 & 16.1 & 16.5 & 15.8 & 16.7 & 18.7 & 14.6 & 18.4 \\
\hline MSD & 8.3 & 9.0 & 2.2 & 3.1 & 5.9 & 6.1 & 5.5 & 5.9 & 2.7 & 5.6 \\
\hline STD & 7.4 & 7.6 & 4.0 & 3.5 & 4.9 & 4.9 & 4.7 & 5.0 & 3.4 & 5.3 \\
\hline
\end{tabular}


要 t mं

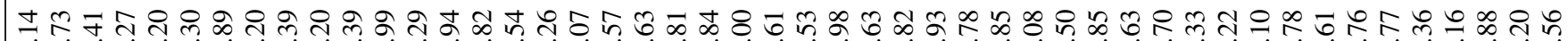

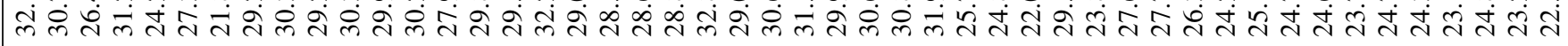

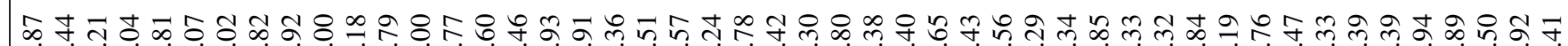

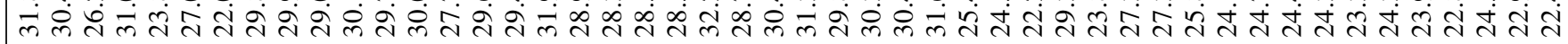

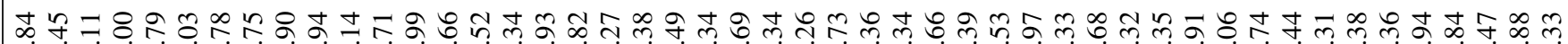
mं

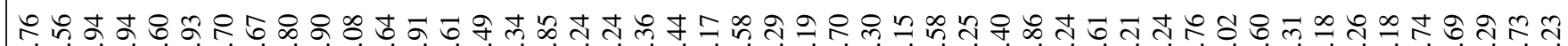

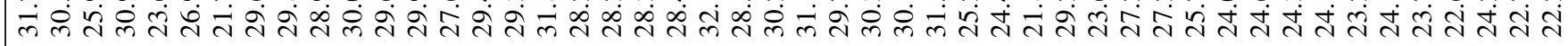

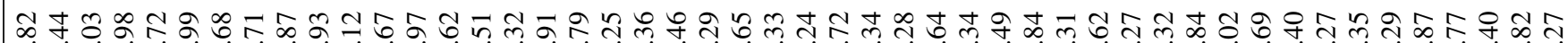

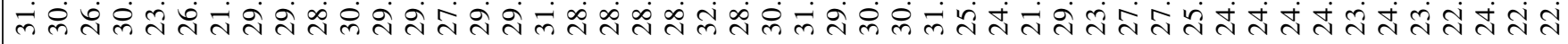

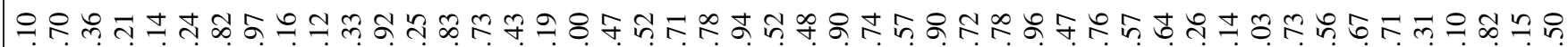

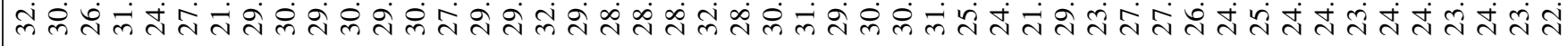

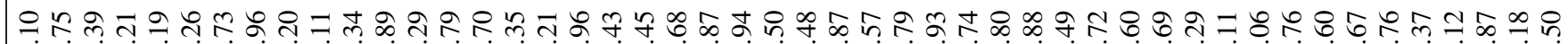
लंळ्र ชิ

ద유

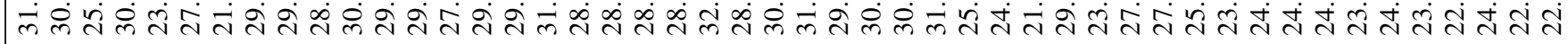

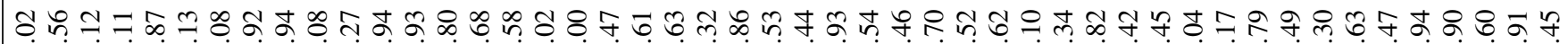

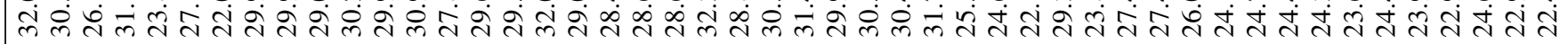

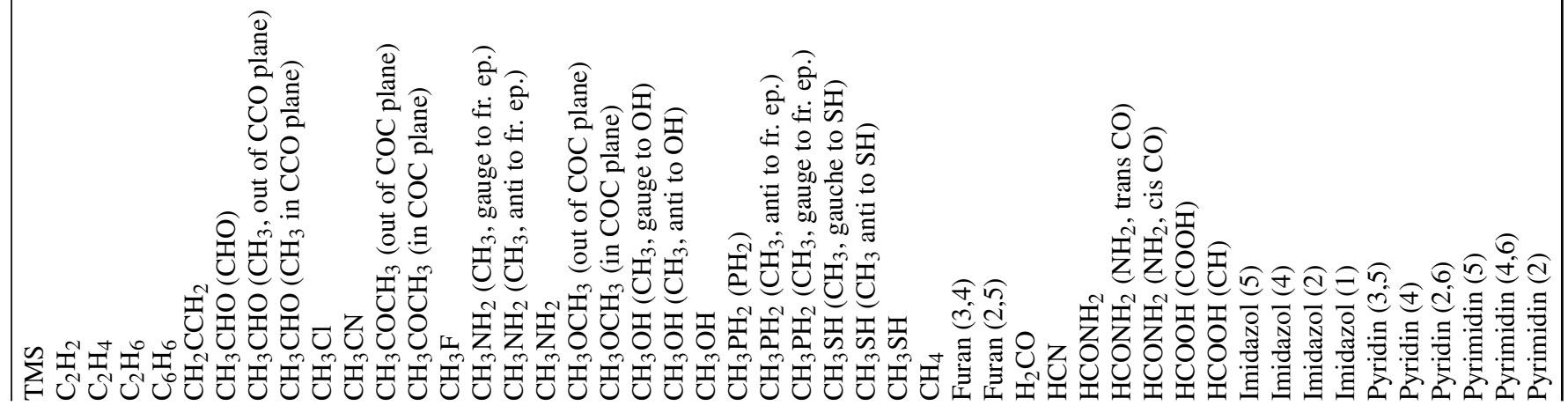




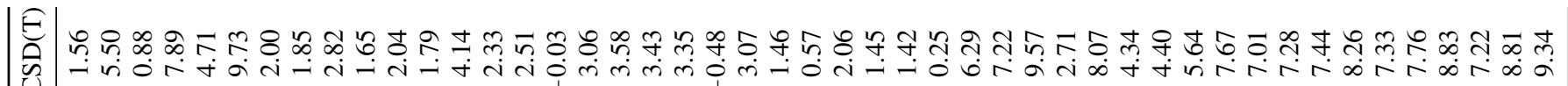

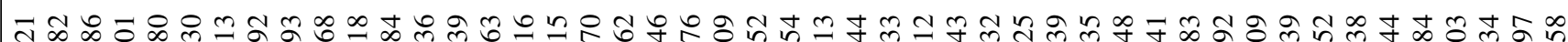

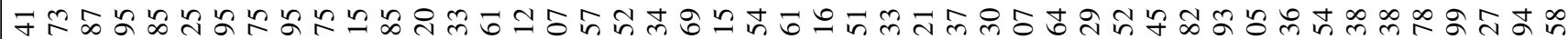

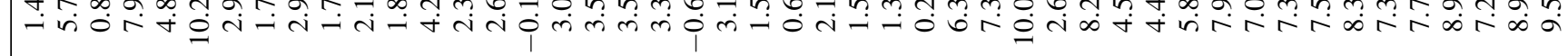

子웅요

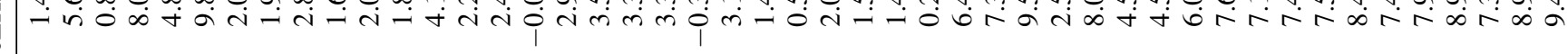

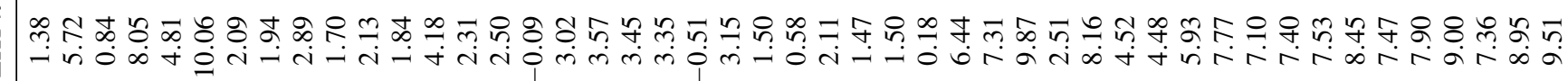

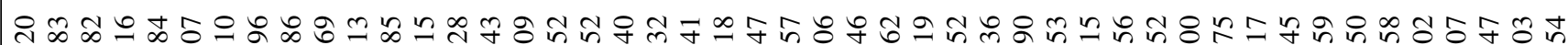

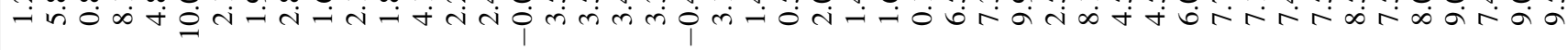

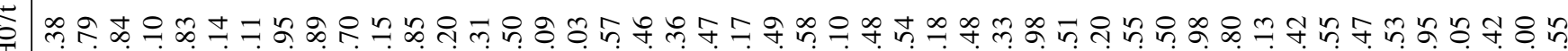

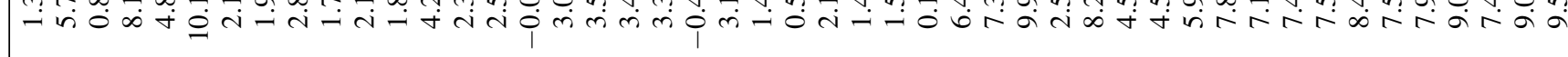

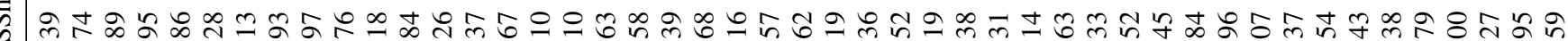

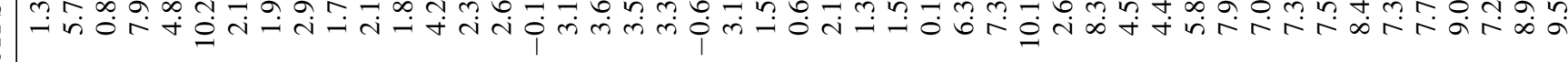

ڤ

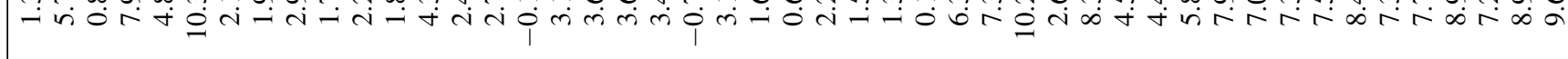

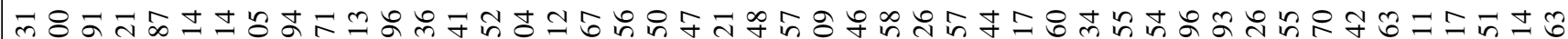

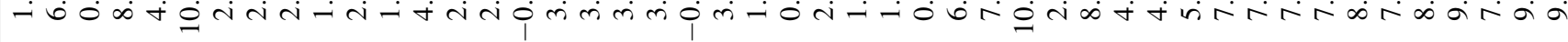

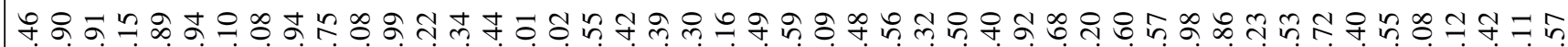

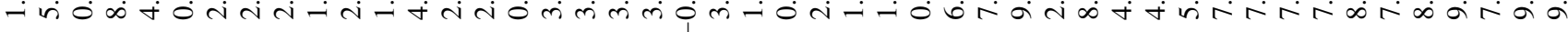

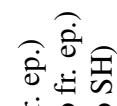

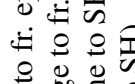

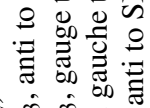

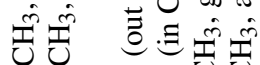

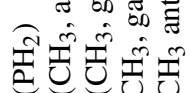

矛 ट्रु 


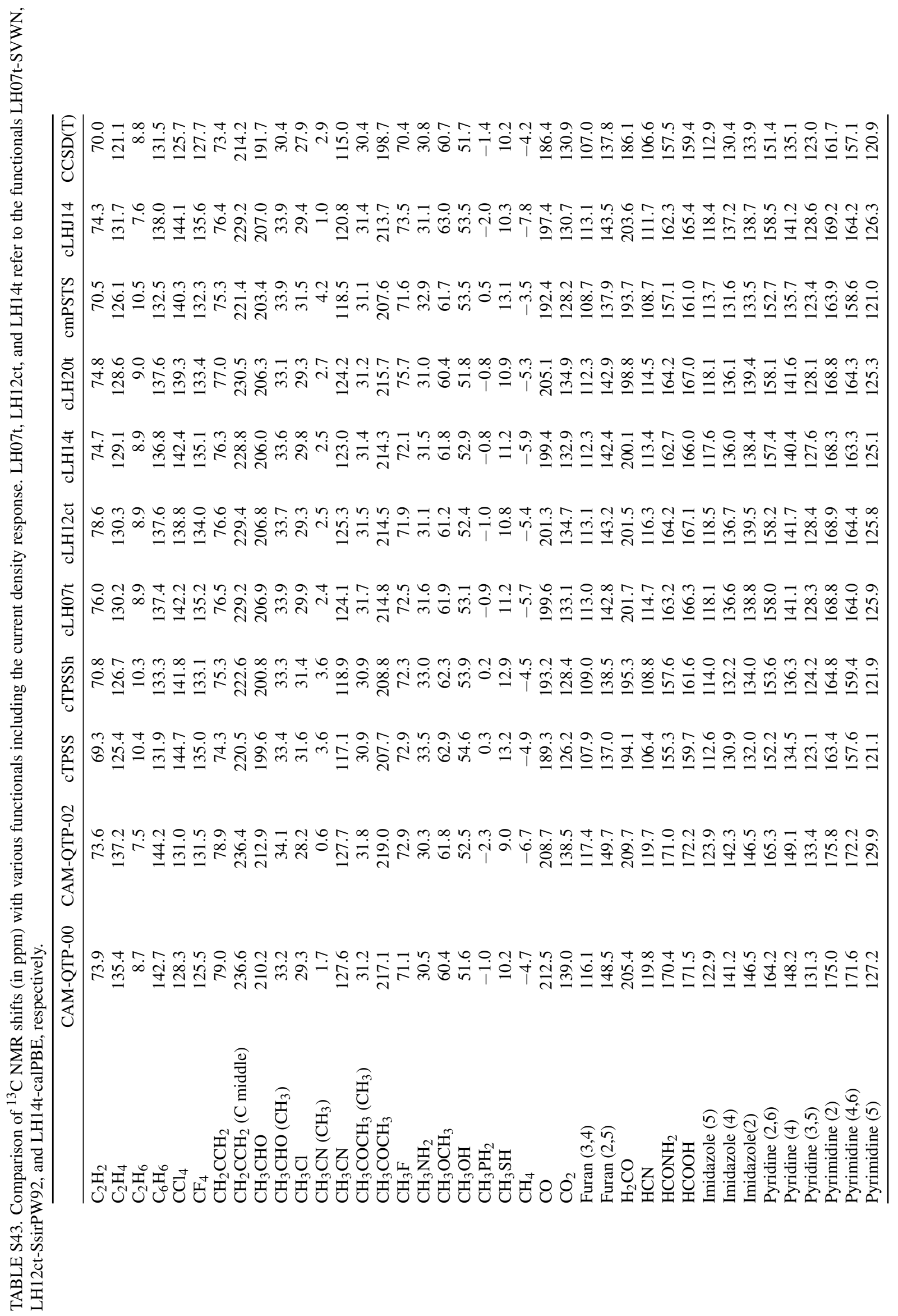




\section{B. NMR Shieldings of ${ }^{1} \mathrm{H},{ }^{13} \mathrm{C},{ }^{15} \mathrm{~N},{ }^{17} \mathrm{O},{ }^{19} \mathrm{~F}$, and ${ }^{31} \mathrm{P}$ Nuclei}

To consider a few additional NMR nuclei of the light elements, another test set [92] was used featuring also N, O, F, and $\mathrm{P}$ compounds. Computational parameters are unchanged compared to the last subsection except for the basis set which was changed to the NMR-tailored pcSseg-4 [93] bases. Structures and reference values are taken from Ref. 92. Errors are measured with respect to the pcSseg-4/CCSD(T) level of theory [92].

Figure S3. Comparison of various functionals for ${ }^{1} \mathrm{H}$ NMR shieldings in ppm. LH07t, LH12ct, and LH14t refer to the functionals LH07tSVWN, LH12ct-SsirPW92, and LH14t-calPBE, respectively MAE, MSD, and STD denote the mean absolute error, mean signed error, and the standard deviation regarding the latter.

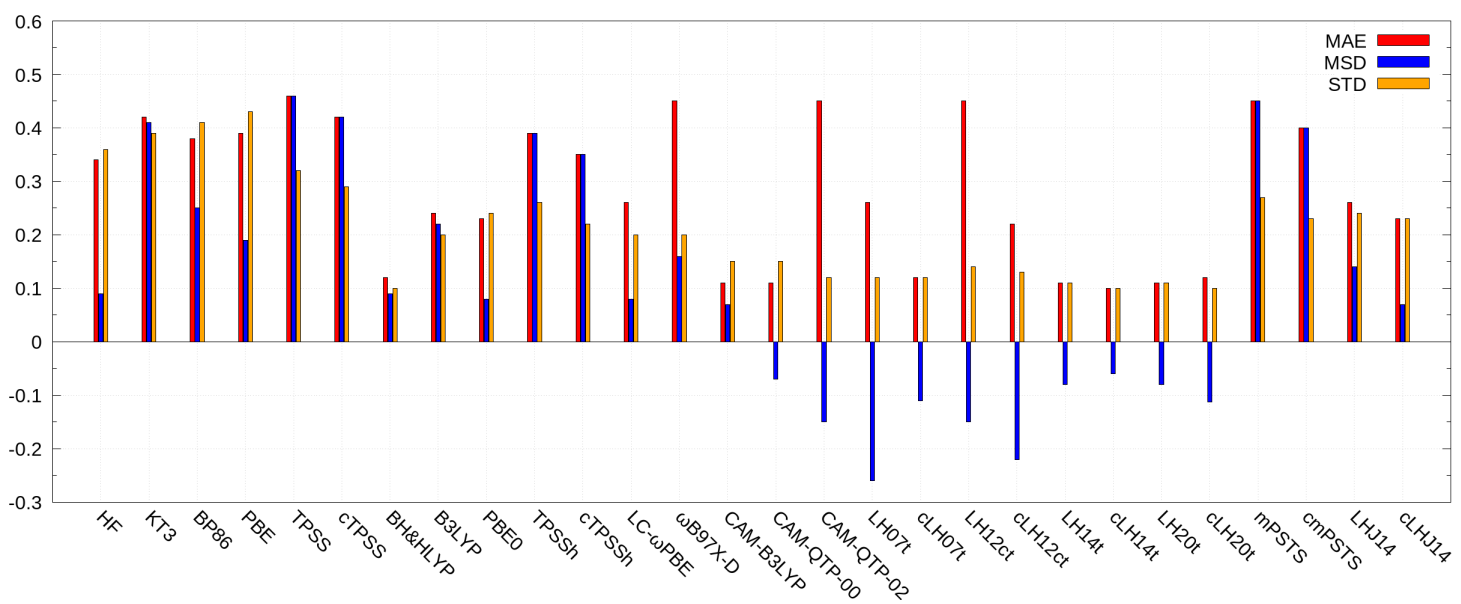

Figure S4. Comparison of various functionals for ${ }^{13} \mathrm{C}$ NMR shieldings in ppm.

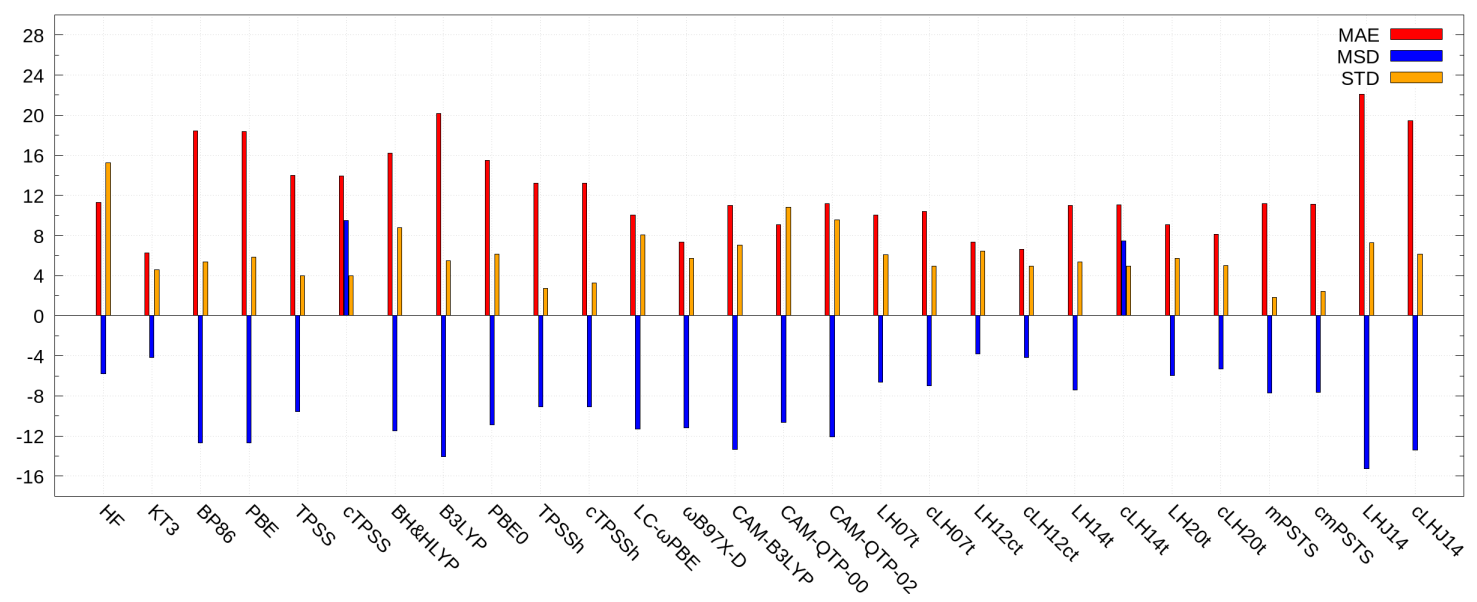


Figure S5. Comparison of various functionals for ${ }^{15} \mathrm{~N},{ }^{17} \mathrm{O},{ }^{19} \mathrm{~F}$, and ${ }^{31} \mathrm{P}$ NMR shieldings.

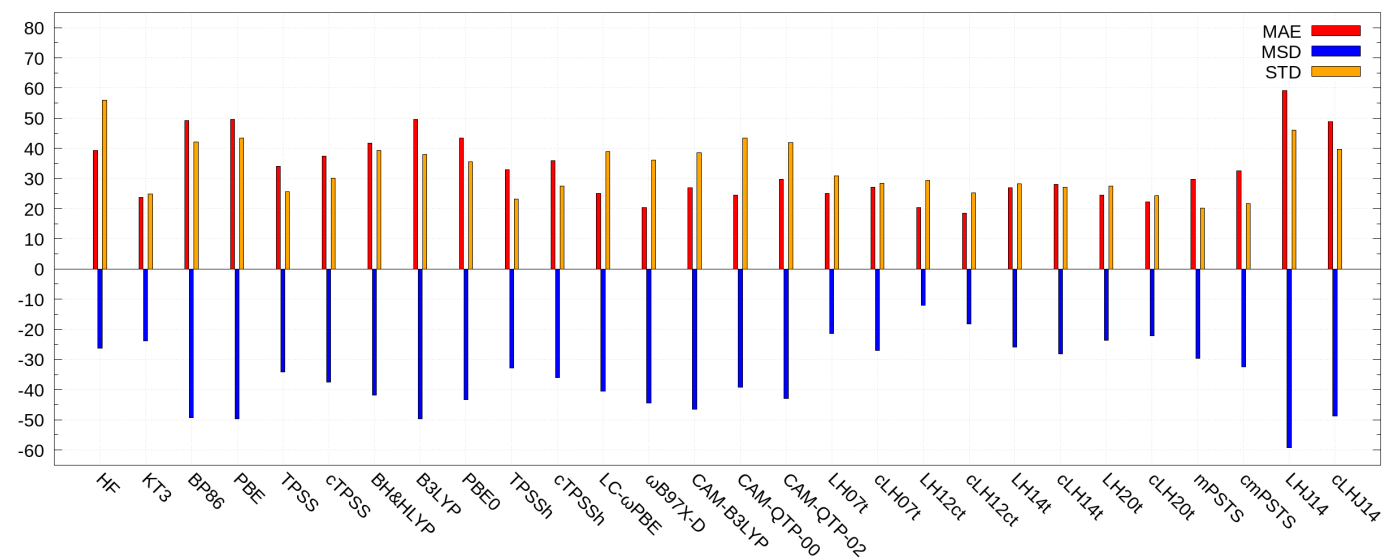

TABLE S44. Statistical evaluation of ${ }^{1} \mathrm{H}$ NMR shieldings (in $\mathrm{ppm}$ ) of various computational methods with respect to CCSD(T). LH07t, LH12ct, and LH14t refer to the functionals LH07t-SVWN, LH12ct-SsirPW92, and LH14t-calPBE, respectively. MAE, MSD, and STD denote the mean absolute error, mean signed error, and the standard deviation regarding the latter.

\begin{tabular}{|c|c|c|c|c|c|c|c|c|c|c|c|c|c|c|c|c|c|c|}
\hline & $\mathrm{HF}$ & KT3 & BP86 & PBE & TPSS & BH\&HLYP & B3LYP & PBE0 & TPSSh & LC- $\omega$ PBE & $\omega \mathrm{B} 97 \mathrm{X}-\mathrm{D}$ & CAM-B3LYP & LH07t & LH12ct & LH14t & LH20t & mPSTS & LHJ14 \\
\hline MAE & 0.34 & 0.42 & 0.38 & 0.39 & 0.46 & 0.12 & 0.24 & 0.23 & 0.39 & 0.26 & 0.45 & 0.11 & 0.26 & 0.45 & 0.11 & 0.11 & 0.45 & 0.26 \\
\hline Max. Deviation & 0.71 & 1.10 & 0.97 & 0.94 & 0.17 & 1.01 & 0.52 & 0.42 & 0.79 & 0.42 & 0.60 & 0.24 & 0.42 & 0.60 & 0.24 & 0.25 & 0.87 & 0.49 \\
\hline MSD & 0.09 & 0.41 & 0.25 & 0.19 & 0.46 & 0.09 & 0.22 & 0.08 & 0.39 & 0.08 & 0.16 & 0.07 & -0.26 & -0.45 & -0.08 & -0.08 & 0.45 & 0.14 \\
\hline STD & 0.36 & 0.39 & 0.41 & 0.43 & 0.32 & 0.10 & 0.20 & 0.24 & 0.26 & 0.20 & 0.20 & 0.15 & 0.12 & 0.14 & 0.11 & 0.11 & 0.27 & 0.24 \\
\hline
\end{tabular}

TABLE S45. Statistical evaluation of ${ }^{13} \mathrm{C}$ NMR shieldings (in ppm) of various computational methods with respect to CCSD(T).

\begin{tabular}{|c|c|c|c|c|c|c|c|c|c|c|c|c|c|c|c|c|c|c|}
\hline & $\mathrm{HF}$ & KT3 & BP86 & PBE & TPSS & BH\&HLYP & B3LYP & PBE0 & TPSSh & LC- $\omega$ PBE & $\omega \mathrm{B} 97 \mathrm{X}-\mathrm{D}$ & CAM-B3LYP & LH07t & LH12ct & LH14t & LH20t & mPSTS & LHJ14 \\
\hline & & 6.27 & 18.44 & 18.39 & & & 20.18 & & & 10.06 & & & & 7.36 & & 9.07 & & 22.06 \\
\hline Max. Deviation & 30.63 & 13.12 & 25.90 & 26.15 & 20.39 & 29.46 & 28.97 & 23.89 & 18.75 & 19.46 & 156.03 & 19.94 & 19.46 & 15.03 & 19.94 & 19.12 & 15.54 & 33.14 \\
\hline MSD & -5.81 & -4.15 & -12.69 & -12.67 & -9.54 & -11.48 & -14.04 & -10.86 & -9.09 & -11.31 & & -13.32 & -6.64 & -3.80 & -7.43 & -5.97 & -7.70 & -15.27 \\
\hline STD & 15.23 & 4.58 & 5.37 & 5.82 & 3.99 & 8.76 & 5.51 & 6.15 & 2.75 & 8.04 & 5.71 & 7.07 & 6.09 & 6.46 & 5.35 & 5.75 & 1.85 & 7.29 \\
\hline
\end{tabular}

TABLE S46. Statistical evaluation of ${ }^{15} \mathrm{~N},{ }^{17} \mathrm{O},{ }^{19} \mathrm{~F}$, and ${ }^{31} \mathrm{P}$ NMR shieldings (in ppm) of various computational methods with respect to $\operatorname{CCSD}(\mathrm{T})$.

\begin{tabular}{|c|c|c|c|c|c|c|c|c|c|c|c|c|c|c|c|c|c|c|}
\hline & $\mathrm{HF}$ & KT3 & BP86 & PBE & TPSS & BH\&HLYP & B3LYP & PBE0 & TPSSh & LC- $\omega$ PBE & $\omega \mathrm{B} 97 \mathrm{X}-\mathrm{D}$ & CAM-B3LYP & LH07t & LH12ct & LH14t & LH20t & mPSTS & LHJ14 \\
\hline MAE & 39.97 & 23.80 & 49.26 & 49.63 & 34.07 & 41.78 & 49.59 & 43.30 & 32.84 & 24.98 & 20.45 & & 24.98 & 20.45 & 26.91 & 24.44 & 29.71 & 59.24 \\
\hline M & & & & & & & & & & & & & & & & & .35 & 186.96 \\
\hline MSD & -26.24 & -23.80 & -49.26 & -49.63 & -34.07 & -41.78 & -49.59 & -43.30 & -32.84 & -40.42 & -44.50 & -46.53 & -21.35 & -12.11 & -25.83 & -23.55 & -29.71 & -59.24 \\
\hline STD & 55.96 & 24.93 & 42.16 & 43.33 & 25.68 & 39.23 & 38.05 & 35.46 & 23.19 & 39.01 & 36.05 & 38.50 & 30.90 & 29.34 & 28.33 & 27.41 & 20.15 & 46.04 \\
\hline
\end{tabular}


TABLE S47. Comparison of ${ }^{1} \mathrm{H}$ NMR shielding constants (in ppm) at various levels. LH07t, LH12ct, and LH14t refer to the functionals LH07t-SVWN, LH12ct-SsirPW92, and LH14t-calPBE, respectively. CCSD(T) results are taken from Ref. 92

\begin{tabular}{|c|c|c|c|c|c|c|c|c|c|c|c|c|c|c|c|c|c|c|c|}
\hline & $\mathrm{HF}$ & KT3 & BP86 & PBE & TPSS & BH\&HLYP & B3LYP & PBE0 & TPSSh & LC- $\omega$ PBE & $\omega \mathrm{B} 97 \mathrm{X}-\mathrm{D}$ & CAM-B3LYP & LH07t & LH12ct & LH14t & LH20t & mPSTS & LHJ14 & $\operatorname{CCSD}(\mathrm{T})$ \\
\hline $\mathrm{PH}_{3}$ & 29.68 & 29.70 & 29.54 & 29.50 & 29.68 & 29.62 & 29.64 & 29.47 & 29.65 & 29.48 & 29.55 & 29.55 & 29.26 & 29.13 & 29.37 & 29.43 & 29.67 & 29.63 & 29.46 \\
\hline $\mathrm{HF}$ & 28.11 & 29.92 & 29.79 & 29.76 & 29.83 & 28.74 & 29.34 & 29.24 & 29.61 & 29.20 & 29.27 & 29.07 & 28.56 & 28.29 & 28.78 & 28.57 & 29.69 & 29.31 & 28.82 \\
\hline $\mathrm{H}_{2} \mathrm{O}$ & 30.49 & 31.39 & 31.26 & 31.23 & 31.38 & 30.74 & 31.05 & 30.97 & 31.26 & 30.89 & 30.98 & 30.84 & 30.48 & 30.28 & 30.67 & 30.58 & 31.33 & 30.98 & 30.65 \\
\hline $\mathrm{NH}_{3}$ & 31.57 & 31.89 & 31.79 & 31.76 & 31.95 & 31.60 & 31.72 & 31.66 & 31.89 & 31.62 & 31.69 & 31.59 & 31.33 & 31.18 & 31.48 & 31.48 & 31.94 & 31.67 & 31.44 \\
\hline $\mathrm{CH}_{4}$ & 31.63 & 31.68 & 31.58 & 31.55 & 31.72 & 31.56 & 31.60 & 31.51 & 31.69 & 31.50 & 31.57 & 31.50 & 31.31 & 31.19 & 31.42 & 31.46 & 31.72 & 31.56 & 31.39 \\
\hline$\left(\mathrm{CH}_{3}\right)_{2} \mathrm{CO}$ & 29.95 & 29.60 & 29.39 & 29.34 & 29.66 & 29.66 & 29.57 & 29.44 & 29.67 & 29.51 & 29.57 & 29.51 & 29.28 & 29.19 & 29.41 & 29.51 & 29.73 & 29.44 & 29.53 \\
\hline Furan $(2,5)$ & 24.28 & 23.97 & 23.87 & 23.80 & 24.15 & 24.03 & 24.00 & 23.83 & 24.12 & 23.90 & 23.96 & 23.91 & 23.68 & 23.52 & 23.84 & 23.93 & 24.18 & 23.90 & 24.03 \\
\hline Furan $(3,4)$ & 25.30 & 25.07 & 24.87 & 24.79 & 25.22 & 25.03 & 25.00 & 24.81 & 25.18 & 24.82 & 24.93 & 24.98 & 24.63 & 24.45 & 24.81 & 24.89 & 25.24 & 24.85 & 25.05 \\
\hline
\end{tabular}

TABLE S48. Comparison of ${ }^{13} \mathrm{C}$ NMR shielding constants (in ppm) at various level. sLH07t, LH12ct, and LH14t refer to the functionals LH07t-SVWN, LH12ct-SsirPW92, and LH14t-calPBE, respectively. CCSD(T) results are taken from Ref. 92.

\begin{tabular}{|c|c|c|c|c|c|c|c|c|c|c|c|c|c|c|c|c|c|c|c|}
\hline & $\mathrm{HF}$ & KT3 & BP86 & PBE & TPSS & BH\&HLYP & B3LYP & PBE0 & TPSSh & LC- $\omega$ PBE & $\omega \mathrm{B} 97 \mathrm{X}-\mathrm{D}$ & CAM-B3LYP & LH07t & LH12ct & LH14t & LH20t & mPSTS & LHJ14 & $\operatorname{CCSD}(\mathrm{T})$ \\
\hline$\left(\mathrm{CH}_{3}\right)_{2} \mathrm{CO}$ & -24.94 & -18.28 & -36.74 & -36.99 & -30.25 & -35.35 & -39.81 & -33.95 & -29.59 & -36.40 & -35.10 & -39.94 & -30.30 & -25.87 & -30.78 & -26.38 & -28.50 & -43.98 & -10.84 \\
\hline & 162.5 & 155.53 & 148.51 & 148.99 & 151.25 & & 148.83 & 153.45 & 152.81 & 156.27 & .98 & 151.58 & 159.38 & 163.67 & 157.64 & 160.27 & 153.86 & 148.50 & 162.88 \\
\hline $\mathrm{CO}$ & -28.07 & 1.75 & -16.35 & -17.24 & -9.05 & -26.90 & -22.94 & -21.33 & -11.29 & -23.64 & -20.58 & -24.28 & -13.20 & -10.87 & -14.04 & -14.29 & -8.83 & -26.57 & 2.56 \\
\hline $\mathrm{CF}_{4}$ & 77.82 & 52.84 & 41.12 & 41.04 & 45.57 & 57.19 & 44.64 & 51.23 & 49.16 & 51.33 & 51.53 & 47.59 & 53.41 & 58.83 & 52.77 & 57.26 & 52.24 & 41.96 & 65.96 \\
\hline $\mathrm{CH}_{4}$ & 195.13 & 192.79 & 189.61 & 190.57 & 188.78 & 191.68 & 188.84 & 192.87 & 189.91 & 195.58 & 192.13 & 191.96 & 199.88 & 203.95 & 197.25 & 198.72 & 189.85 & 190.00 & 199.39 \\
\hline Furan $(2,5)$ & 36.85 & 43.38 & 29.99 & 30.00 & 35.65 & 29.18 & 26.50 & 31.69 & 35.78 & 29.31 & 30.32 & 26.18 & 38.02 & 42.06 & 37.27 & 39.39 & 38.19 & 24.59 & 47.36 \\
\hline Furan $(3,4)$ & 74.51 & 77.12 & 63.72 & 63.90 & 69.22 & 65.45 & 61.64 & 66.43 & 69.74 & 65.19 & 65.33 & 62.25 & 72.33 & 76.40 & 71.70 & 74.07 & 71.59 & 60.06 & 81.67 \\
\hline
\end{tabular}

TABLE S49. Comparison of ${ }^{15} \mathrm{~N},{ }^{17} \mathrm{O},{ }^{19} \mathrm{~F}$, and ${ }^{31} \mathrm{P}$ NMR shielding constants (in ppm) at various levels. LH07t, LH12ct, and LH14t refer to the functionals LH07t-SVWN, LH12ct-SsirPW92, and LH14t-calPBE, respectively. CCSD(T) results are taken from Ref. 92

\begin{tabular}{|c|c|c|c|c|c|c|c|c|c|c|c|c|c|c|c|c|c|c|c|}
\hline & $\mathrm{HF}$ & KT3 & BP86 & PBE & TPSS & BH\&HLYP & B3LYP & PBE0 & TPSSh & LC- $\omega$ PBE & $\omega \mathrm{B} 97 \mathrm{X}-\mathrm{D}$ & CAM-B3LYP & LH07t & LH12ct & LH14t & LH20t & mPSTS & LHJ14 & $\operatorname{CCSD}(\mathrm{T})$ \\
\hline PN & -511.98 & -375.19 & -418.86 & -419.72 & -399.46 & -476.24 & -448.36 & -446.51 & -411.09 & -442.04 & -451.54 & -451.64 & -402.49 & -396.78 & -408.09 & -417.34 & -407.97 & -460.36 & -344.71 \\
\hline $\mathrm{N}_{2}$ & -116.14 & -66.29 & -87.91 & -88.59 & -79.28 & -106.14 & -97.32 & -96.95 & -83.23 & -95.08 & -97.81 & -97.90 & -81.69 & -78.83 & -83.36 & -85.94 & -81.33 & -102.40 & -61.16 \\
\hline NNO & -35.81 & 10.11 & -1.69 & -1.22 & 1.55 & -24.15 & -14.54 & -9.89 & -2.26 & -13.07 & -13.99 & -17.92 & -1.54 & 0.68 & -3.34 & -6.22 & -0.85 & -14.60 & 11.74 \\
\hline NO & 60.96 & 8.96 & 88.65 & .21 & 91.12 & 70.54 & .03 & 81.60 & 88.03 & 81.51 & 79.32 & 78.06 & 94.47 & 97.78 & 91.38 & 88.74 & 88.87 & 76.94 & 106.22 \\
\hline $\mathrm{H}_{\mathrm{O}}$ & 262.09 & 261.85 & 260.79 & 261.89 & 259.27 & & 259.70 & 262.84 & 260.00 & 265.24 & 261.76 & & 275.86 & 280.45 & 270.34 & 270.50 & 259.04 & 258.66 & 270.40 \\
\hline $\mathrm{OF}_{2}$ & -449.03 & -555.13 & -631.06 & -635.45 & -562.68 & -531.00 & -594.65 & -567.82 & -543.30 & -545.39 & -562.15 & -572.28 & -539.10 & -512.41 & -538.33 & -514.20 & -527.67 & -633.28 & -446.32 \\
\hline$\left(\mathrm{CH}_{3}\right)_{2} \mathrm{CO}$ & -333.41 & -298.48 & -348.82 & -352.37 & -320.55 & -354.12 & -356.16 & -357.38 & -324.76 & -353.15 & -357.91 & -356.38 & -316.46 & -306.12 & -322.62 & -328.08 & -321.14 & -373.26 & -297.91 \\
\hline $\mathrm{CO}$ & -92.0 & -61.02 & -83.69 & -84.92 & -71.16 & -91.33 & -88.12 & -89.38 & -73.83 & -81.77 & -90.89 & -86.83 & -65.00 & -60.25 & -68.36 & -68.82 & -72.03 & -96.47 & -55.42 \\
\hline Furan & 58.14 & 29.74 & 11.84 & 10.20 & 22.45 & 8.75 & 15.02 & 19.10 & 24.85 & 32.25 & 23.06 & & 37.53 & 44.73 & & 38.72 & 27.18 & 12.47 & 64.82 \\
\hline NNO & 173.25 & 173 & 173.02 & & & & & 172.67 & & & & & 1.34 & 196.50 & & .70 & 2.26 & .19 & 8.77 \\
\hline $\mathrm{H}_{2} \mathrm{O}$ & 327.58 & 326.29 & 327.55 & 328.28 & 326.56 & 7.56 & 326.56 & 328.98 & 327.14 & 333.17 & 328.56 & 330.16 & 349.52 & 354.89 & 340.85 & 340.06 & 325.55 & 323.25 & 337.63 \\
\hline$F_{2}$ & -174.55 & -233.02 & -271.46 & -274.22 & -237.36 & -228.23 & -260.45 & -247.88 & -230.89 & -239.74 & -250.60 & -248.13 & -216.13 & -198.69 & -220.79 & -209.61 & -224.84 & -278.56 & -192.76 \\
\hline $\mathrm{OF}_{2}$ & & -73.42 & & -94.37 & -73.65 & -39.64 & -76.86 & -59.60 & -61.75 & & & & 34.42 & -16.40 & & -31.15 & -54.93 & -89.96 & -24.28 \\
\hline $\mathrm{PF}_{3}$ & 255.25 & 195.77 & & & .14 & & 8.89 & 2.93 & & & & & 226.78 & 237.81 & & 226.68 & 208.28 & 184.21 & 231.81 \\
\hline $\mathrm{CF}_{4}$ & 281.91 & 241.28 & 232.25 & 231.05 & 241.13 & 257.05 & 241.29 & 245.67 & 246.11 & 248.70 & 248.19 & 244.36 & 262.85 & 271.37 & 257.04 & 260.70 & 248.98 & 230.19 & 267.58 \\
\hline $\mathrm{HF}^{4}$ & 414.32 & 410.60 & 411.17 & 411.42 & 411.23 & & 411.50 & 412.90 & 411.97 & 416.62 & 412.76 & 414.39 & 437.05 & 442.81 & 426.93 & 425.83 & 410.10 & 405.60 & 419.91 \\
\hline PN & -110.40 & 34.20 & -47.68 & -47.15 & -8.68 & -94.87 & -71.27 & -72.03 & -21.03 & -10 & & & -45.76 & -41.15 & & -47.30 & & .75 & 51.61 \\
\hline $\mathrm{PF}_{3}$ & & & & & & & & 182.80 & & & & & 189.28 & & & & & & 224.80 \\
\hline $\mathrm{PH}_{3}$ & 583.53 & 588.28 & 569.07 & 575.63 & 570.38 & 569.71 & 561.38 & 582.47 & 573.72 & 580.10 & 574.91 & 566.70 & 599.47 & 615.87 & 589.58 & 593.24 & 574.24 & 563.90 & 604.51 \\
\hline
\end{tabular}


TABLE S50. Statistical evaluation of ${ }^{1} \mathrm{H}$ NMR shieldings (in ppm) of various computational methods with respect to CCSD(T). LH07t, LH12ct, and LH14t refer to the functionals LH07t-SVWN, LH12ct-SsirPW92, and LH14t-calPBE, respectively. MAE, MSD, and STD denote the mean absolute error, mean signed error, and the standard deviation regarding the latter.

\begin{tabular}{lccccrrrrrr}
\hline & CAM-QTP-00 & CAM-QTP-02 & cTPSS & cTPSSh & cLH07t & cLH12ct & cLH14t & cLH20t & cmPSTS & cLHJ14 \\
\hline MAE & 0.11 & 0.45 & 0.42 & 0.35 & 0.12 & 0.22 & 0.10 & 0.12 & 0.40 & 0.23 \\
Max. Deviation & 0.25 & 0.87 & 0.92 & 0.71 & 0.30 & 0.42 & 0.23 & 0.29 & 0.78 & 0.42 \\
MSD & -0.07 & -0.15 & 0.42 & 0.35 & -0.11 & -0.22 & -0.06 & -0.12 & 0.40 & 0.07 \\
STD & 0.15 & 0.12 & 0.29 & 0.22 & 0.12 & 0.13 & 0.10 & 0.10 & 0.23 & 0.23 \\
\hline
\end{tabular}

TABLE S51. Statistical evaluation of ${ }^{13} \mathrm{C}$ NMR shieldings (in ppm) of various computational methods with respect to CCSD(T).

\begin{tabular}{|c|c|c|c|c|c|c|c|c|c|c|}
\hline & CAM-QTP-00 & CAM-QTP-02 & cTPSS & cTPSSh & $\mathrm{cLH} 07 \mathrm{t}$ & $\mathrm{cLH} 12 \mathrm{ct}$ & $\mathrm{cLH} 14 \mathrm{t}$ & $\mathrm{cLH} 20 \mathrm{t}$ & cmPSTS & cLHJ14 \\
\hline MAE & 9.07 & 11.21 & 13.92 & 13.19 & 10.38 & 6.63 & 11.05 & 8.11 & 11.14 & 19.43 \\
\hline Max. Deviation & 19.12 & 15.54 & 20.16 & 19.45 & 19.05 & 14.24 & 19.62 & 17.26 & 16.24 & 28.86 \\
\hline MSD & -10.66 & -12.09 & -9.47 & -9.05 & -6.97 & -4.16 & -7.45 & -5.30 & -7.62 & -13.42 \\
\hline STD & 10.84 & 9.54 & 3.96 & 3.28 & 4.95 & 4.93 & 4.95 & 5.01 & 2.40 & 6.13 \\
\hline
\end{tabular}

TABLE S52. Statistical evaluation of ${ }^{15} \mathrm{~N},{ }^{17} \mathrm{O},{ }^{19} \mathrm{~F}$, and ${ }^{31} \mathrm{P}$ NMR shieldings (in ppm) of various computational methods with respect to $\operatorname{CCSD}(\mathrm{T})$.

\begin{tabular}{|c|c|c|c|c|c|c|c|c|c|c|}
\hline & CAM-QTP-00 & CAM-QTP-02 & cTPSS & cTPSSh & $\mathrm{cLH} 07 \mathrm{t}$ & cLH12ct & cLH14t & $\mathrm{cLH} 20 \mathrm{t}$ & cmPSTS & cLHJ14 \\
\hline MAE & 24.44 & 29.71 & 37.41 & 35.93 & 27.08 & 18.52 & 28.06 & 22.21 & 32.48 & 48.80 \\
\hline Max. Deviation & 98.91 & 81.35 & 134.15 & 112.57 & 100.06 & 93.55 & 96.14 & 94.16 & 96.09 & 156.58 \\
\hline MSD & -39.24 & -43.01 & -37.41 & -35.93 & -27.08 & -18.27 & -28.06 & -22.20 & -32.48 & -48.80 \\
\hline STD & 43.46 & 41.87 & 30.09 & 27.57 & 28.45 & 25.29 & 27.05 & 24.23 & 24.47 & 39.66 \\
\hline
\end{tabular}


TABLE S53. Comparison of ${ }^{1} \mathrm{H}$ NMR shielding constants (in ppm) at various levels. LH07t, LH12ct, and LH14t refer to the functionals LH07t-SVWN, LH12ct-SsirPW92, and LH14t-calPBE, respectively. CCSD(T) results are taken from Ref. 92

\begin{tabular}{|c|c|c|c|c|c|c|c|c|c|c|c|}
\hline & CAM-QTP-00 & CAM-QTP-02 & cTPSS & cTPSSh & cLH07t & cLH12ct & cLH14t & $\mathrm{cLH} 20 \mathrm{t}$ & cmPSTS & cLHJ14 & $\operatorname{CCSD}(\mathrm{T})$ \\
\hline $\mathrm{PH}_{3}$ & 29.52 & 29.40 & 29.69 & 29.66 & 29.38 & 29.29 & 29.40 & 29.41 & 29.68 & 29.54 & 29.46 \\
\hline $\mathrm{HF}$ & 28.45 & 28.64 & 29.74 & 29.53 & 28.77 & 28.57 & 28.82 & 28.53 & 29.60 & 29.24 & 28.82 \\
\hline $\mathrm{H}_{2} \mathrm{O}$ & 30.54 & 30.55 & 31.29 & 31.18 & 30.65 & 30.52 & 30.69 & 30.52 & 31.25 & 30.92 & 30.65 \\
\hline $\mathrm{NH}_{3}$ & 31.47 & 31.41 & 31.88 & 31.82 & 31.47 & 31.38 & 31.49 & 31.41 & 31.87 & 31.61 & 31.44 \\
\hline $\mathrm{CH}_{4}$ & 31.47 & 31.38 & 31.70 & 31.67 & 31.42 & 31.35 & 31.43 & 31.40 & 31.70 & 31.50 & 31.39 \\
\hline$\left(\mathrm{CH}_{3}\right)_{2} \mathrm{CO}$ & 23.95 & 23.77 & 29.64 & 29.65 & 29.40 & 29.35 & 29.43 & 29.47 & 29.71 & 29.38 & 29.53 \\
\hline Furan $(2,5)$ & 23.95 & 23.77 & 24.16 & 24.13 & 23.81 & 23.69 & 23.88 & 23.92 & 24.18 & 23.85 & 24.03 \\
\hline Furan $(3,4)$ & 24.92 & 24.71 & 25.18 & 25.15 & 24.75 & 24.63 & 24.82 & 24.86 & 25.20 & 24.82 & 25.05 \\
\hline
\end{tabular}

TABLE S54. Comparison of ${ }^{13} \mathrm{C}$ NMR shielding constants (in ppm) at various level. sLH07t, LH12ct, and LH14t refer to the functionals LH07t-SVWN, LH12ct-SsirPW92, and LH14t-calPBE, respectively. CCSD(T) results are taken from Ref. 92.

\begin{tabular}{lcrrrrrrrrrr}
\hline & CAM-QTP-00 & CAM-QTP-02 & cTPSS & cTPSSh & cLH07t & cLH12ct & cLH14t & cLH20t & cmPSTS & cLHJ14 & CCSD(T) \\
\hline$\left(\mathrm{CH}_{3}\right)_{2} \mathrm{CO}$ & -34.96 & -39.08 & -31.00 & -30.29 & -29.89 & -25.08 & -30.46 & -28.10 & -27.08 & -39.70 & -10.84 \\
$\left(\mathrm{CH}_{3}\right)_{2} \mathrm{CO}$ & 156.77 & 155.14 & 151.89 & 153.38 & 158.06 & 161.96 & 157.32 & 160.44 & 154.50 & 149.70 & 162.88 \\
$\mathrm{CO}$ & -28.72 & -26.92 & -11.10 & -13.21 & -13.21 & -10.26 & -13.95 & -12.47 & -10.69 & -21.05 & 2.56 \\
$\mathrm{CF}_{4}$ & 60.85 & 53.33 & 46.10 & 49.63 & 54.00 & 59.49 & 53.11 & 58.18 & 52.69 & 43.42 & 65.96 \\
$\mathrm{CH}_{4}$ & 194.32 & 195.60 & 189.75 & 190.75 & 197.16 & 200.46 & 196.34 & 198.40 & 190.85 & 191.13 & 199.39 \\
Furan (2,5) & 29.45 & 26.61 & 36.13 & 36.22 & 38.16 & 42.18 & 37.52 & 40.66 & 38.66 & 27.12 & 47.36 \\
Furan (3,4) & 66.41 & 63.68 & 69.74 & 70.20 & 72.02 & 75.96 & 71.78 & 75.07 & 72.10 & 62.38 & 81.67 \\
\hline
\end{tabular}

TABLE S55. Comparison of ${ }^{15} \mathrm{~N},{ }^{17} \mathrm{O},{ }^{19} \mathrm{~F}$, and ${ }^{31} \mathrm{P}$ NMR shielding constants (in ppm) at various levels. LH07t, LH12ct, and LH14t refer to the functionals LH07t-SVWN, LH12ct-SsirPW92, and LH14t-calPBE, respectively. CCSD(T) results are taken from Ref. 92

\begin{tabular}{lcrrrrrrrrrr}
\hline & CAM-QTP-00 & CAM-QTP-02 & cTPSS & cTPSSh & cLH07t & cLH12ct & cLH14t & cLH20t & cmPSTS & cLHJ14 & CCSD(T) \\
\hline $\mathrm{PN}$ & -481.18 & -463.45 & -411.95 & -422.83 & -406.36 & -397.87 & -408.36 & -407.70 & -419.48 & -434.29 & -344.71 \\
$\mathrm{~N}_{2}$ & -108.42 & -101.81 & -82.40 & -86.14 & -83.43 & -80.06 & -83.73 & -82.81 & -84.08 & -93.39 & -61.16 \\
$\mathrm{NNO}$ & -27.08 & -22.49 & -1.34 & -2.46 & -1.98 & 0.32 & -3.30 & -4.60 & -1.00 & -11.07 & 11.74 \\
$\mathrm{NNO}$ & 68.46 & 74.96 & 90.74 & 87.63 & 92.44 & 95.41 & 90.67 & 89.66 & 88.60 & 81.21 & 106.22 \\
$\mathrm{NH}_{3}$ & 263.58 & 266.28 & 259.96 & 260.56 & 267.77 & 270.66 & 267.00 & 268.67 & 259.98 & 262.72 & 270.40 \\
$\mathrm{OF}_{2}$ & -513.37 & -542.27 & -580.47 & -558.89 & -541.52 & -511.37 & -538.10 & -504.72 & -542.41 & -602.90 & -446.32 \\
$\left.\mathrm{CH}_{3}\right)_{2} \mathrm{CO}$ & -354.08 & -359.73 & -329.97 & -333.52 & -321.76 & -310.33 & -324.31 & -322.74 & -329.53 & -353.22 & -297.91 \\
$\mathrm{CO}$ & -91.80 & -88.74 & -75.63 & -78.00 & -70.91 & -66.07 & -70.55 & -66.26 & -75.95 & -82.40 & -55.42 \\
$\mathrm{Furan}$ & 36.21 & 30.04 & 22.51 & 24.89 & 35.78 & 42.64 & 34.95 & 40.20 & 27.34 & 18.08 & 64.82 \\
$\mathrm{NNO}$ & 174.75 & 182.21 & 171.99 & 171.69 & 185.89 & 190.02 & 183.49 & 183.48 & 171.08 & 174.20 & 198.77 \\
$\mathrm{H}_{2} \mathrm{O}$ & 330.33 & 333.70 & 326.89 & 327.35 & 335.28 & 337.79 & 334.49 & 336.30 & 326.42 & 330.89 & 337.63 \\
$\mathrm{~F}_{2}$ & -217.09 & -230.38 & -245.90 & -238.46 & -223.13 & -205.51 & -223.35 & -206.55 & -231.76 & -260.74 & -192.76 \\
$\mathrm{OF}_{2}$ & -26.36 & -43.74 & -74.26 & -62.25 & -39.67 & -22.84 & -43.97 & -30.68 & -55.09 & -81.74 & -24.28 \\
$\mathrm{PF}_{3}$ & 225.71 & 209.17 & 196.35 & 203.57 & 218.52 & 227.74 & 216.52 & 224.42 & 208.75 & 191.19 & 231.81 \\
$\mathrm{CF}_{4}$ & 260.58 & 249.89 & 241.81 & 246.66 & 255.79 & 262.70 & 253.73 & 258.90 & 249.83 & 235.82 & 267.58 \\
$\mathrm{HF}$ & 415.58 & 417.26 & 411.48 & 412.09 & 418.40 & 420.35 & 417.99 & 419.99 & 411.15 & 414.98 & 419.91 \\
$\mathrm{PN}$ & -112.64 & -113.96 & -20.43 & -32.08 & -48.45 & -41.94 & -44.53 & -42.55 & -27.27 & -73.84 & 51.61 \\
$\mathrm{PF}_{3}$ & 203.20 & 176.63 & 180.53 & 187.91 & 189.33 & 204.68 & 188.84 & 201.13 & 195.64 & 159.63 & 224.80 \\
$\mathrm{PH}_{3}$ & 575.29 & 576.49 & 573.97 & 576.85 & 590.74 & 603.72 & 586.59 & 591.31 & 577.88 & 564.84 & 604.51 \\
\hline
\end{tabular}




\section{NMR Shieldings and Shifts of Transition-Metal Oxo Compounds}

All structures of the compounds $\mathrm{MO}_{4}^{2-}(\mathrm{M}=\mathrm{Cr}, \mathrm{Mo}, \mathrm{W}), \mathrm{MO}_{4}^{-}(\mathrm{M}=\mathrm{Mn}, \mathrm{Tc} \mathrm{Re})$, and $\mathrm{MO}_{4}(\mathrm{M}=\mathrm{Fe}, \mathrm{Ru}$, Os $)$ were optimized with the scalar-relativistic exact two-component (X2C) Hamiltonian utilizing the diagonal local approximation to the unitary decoupling transformation (DLU) [94-96]. Geometries were considered converged when the energy change between optimization steps falls below $10^{-6} \mathrm{E}_{\mathrm{h}}$. The optimized structures were confirmed to be (local) minima on the potential energy surface by numerical frequency calculations. The conductor-like screening model (COSMO) [97, 98] was applied with the default parameters to compensate the negative charge. SCF energies were converged up to $10^{-9} \mathrm{E}_{\mathrm{h}}$. Tailored grids (gridsize $4 \mathrm{a}$ ) and basis sets (x2c-TZVPall-s) are employed [99]. The finite nucleus model is utilized in all SCF and gradient calculations [96]. The finite nucleus model is employed for both the scalar potential and the vector potential in the NMR calculations [100]. The corresponding parameters are taken from Ref. 101. A threshold of $10^{-7}$ a.u. for the norm of the residuum is used to indicate the convergence of the CPKS equations. NMR shifts are listed with respect to $\mathrm{H}_{2} \mathrm{O}$. The experimental values are taken from Ref. 102 but converted to a more suitable scale as outlined in Refs. 103 and [104]. For these transition-metal oxo compounds, scalar-relativistic approaches are sufficient [105] but solvent effects can amount to $20-30 \mathrm{ppm}$ for $\mathrm{MoO}_{4}^{-}$and $\mathrm{WO}_{4}^{2-}[103]$.

\section{NMR Data}

TABLE S56. Comparison of ${ }^{17} \mathrm{O}$ NMR shielding constants (in ppm) at various levels. LH07, LH12ct, and LH14t refer to the functionals LH07-SVWN, LH12ct-SsirPW92, and LH14t-calPBE, respectively.

\begin{tabular}{|c|c|c|c|c|c|c|c|c|c|c|c|c|c|c|c|c|c|c|}
\hline & HF & KT3 & BP86 & PBE & TPSS & BH\&HLYP & B3LYP & PBE0 & TPSSh & LC- $\omega$ PBE & $\omega \mathrm{B} 97 \mathrm{X}-\mathrm{D}$ & CAM-B3LYP & LH07t & LH12ct & LH14t & LH20t & mPSTS & LHJ14 \\
\hline $\mathrm{H}_{2} \mathrm{O}$ & 337 & 329 & 320 & 322 & 321 & 333 & 325 & 329 & 324 & 333 & 329 & 330 & 348 & 355 & 340 & 342 & 323 & 323 \\
\hline $\mathrm{CrO}_{4}^{2-}$ & -720 & -413 & -453 & -450 & -435 & -598 & -439 & -502 & -457 & -438 & -496 & -512 & -460 & -461 & -468 & -490 & -457 & -505 \\
\hline $\mathrm{MoO}_{4}^{2-}$ & -225 & -221 & -247 & -245 & -238 & -254 & -267 & -241 & -238 & -187 & -227 & -237 & -213 & -202 & -221 & -221 & -237 & -256 \\
\hline $\mathrm{WO}_{4}^{2-}$ & -102 & -101 & -130 & -128 & -121 & -127 & -141 & -120 & -119 & -74 & -103 & -116 & -97 & -85 & -103 & -102 & -118 & -136 \\
\hline $\mathrm{MnO}_{4}^{-}$ & -1742 & -702 & -747 & -742 & -724 & -1150 & -938 & -785 & -785 & -839 & -911 & -939 & -829 & -855 & -842 & -905 & -793 & -869 \\
\hline $\mathrm{TcO}_{4}^{-}$ & -538 & -426 & -458 & -454 & -448 & -534 & -516 & -481 & -461 & -421 & -473 & -487 & -444 & -438 & -453 & -465 & -462 & -489 \\
\hline $\mathrm{ReO}_{4}^{-}$ & -300 & -254 & -289 & -285 & -278 & -314 & -318 & -289 & -281 & -234 & -273 & -289 & -261 & -251 & -269 & -271 & -281 & -302 \\
\hline $\mathrm{FeO}_{4}^{4}$ & -5547 & -1126 & -1173 & -1166 & -1141 & -2628 & -1678 & -1658 & -1308 & -1726 & -1762 & -1844 & -1511 & -1657 & -1545 & -1794 & -1343 & -1508 \\
\hline $\mathrm{RuO}_{4}$ & -1284 & -713 & -751 & -745 & -740 & -1073 & -916 & -872 & -790 & -817 & -883 & -912 & -807 & -824 & -823 & -877 & -801 & -852 \\
\hline $\mathrm{OsO}_{4}$ & -660 & -454 & -496 & -489 & -482 & -614 & -571 & -533 & -501 & -469 & -523 & -547 & -493 & -489 & -503 & -519 & -505 & -536 \\
\hline
\end{tabular}

TABLE S57. Comparison of ${ }^{17} \mathrm{O}$ NMR shifts (in ppm) at various levels. Experimental results are taken from Ref. 102

\begin{tabular}{|c|c|c|c|c|c|c|c|c|c|c|c|c|c|c|c|c|c|c|c|}
\hline & $\mathrm{HF}$ & KT3 & BP86 & PBE & TPSS & BH\&HLYP & B3LYP & PBE0 & TPSSh & LC- $\omega$ PBE & $\omega \mathrm{B} 97 \mathrm{X}-\mathrm{D}$ & CAM-B3LYP & LH07t & LH12ct & LH14t & LH20t & mPSTS & LHJ14 & Exp. \\
\hline $\mathrm{CrO}_{4}^{2-}$ & 1058 & 742 & 773 & 772 & 755 & 930 & 764 & 831 & 781 & 772 & 826 & 842 & 808 & 815 & 808 & 832 & 780 & 828 & 871 \\
\hline $\mathrm{MoO}_{4}^{2-}$ & 563 & 550 & 567 & 567 & 559 & 586 & 592 & 570 & 562 & 521 & 556 & 567 & 561 & 557 & 561 & 563 & 559 & 579 & 576 \\
\hline $\mathrm{WO}_{4}^{2-}$ & 439 & 430 & 450 & 449 & 442 & 459 & 466 & 449 & 443 & 407 & 433 & 446 & 445 & 440 & 443 & 444 & 441 & 459 & 456 \\
\hline $\mathrm{MnO}_{4}^{-}$ & 2079 & 1031 & 1067 & 1064 & 1045 & 1483 & 1263 & 1114 & 1109 & 1172 & 1240 & 1269 & 1177 & 1210 & 1182 & 1246 & 1116 & 1192 & 11255 \\
\hline $\mathrm{TcO}_{4}^{-}$ & 876 & 755 & 778 & 776 & 769 & 867 & 841 & 810 & 785 & 754 & 802 & 817 & 792 & 792 & 793 & 807 & 785 & 812 & 786 \\
\hline $\mathrm{ReO}_{4}^{-}$ & 637 & 583 & 609 & 606 & 599 & 647 & 643 & 618 & 605 & 567 & 602 & 619 & 609 & 606 & 608 & 612 & 604 & 625 & 605 \\
\hline $\mathrm{FeO}_{4}$ & 5885 & 1455 & 1493 & 1487 & 1462 & 2960 & 2003 & 1987 & 1632 & 2059 & 2091 & 2174 & 1859 & 2012 & 1884 & 2135 & 1665 & 1831 & - \\
\hline $\mathrm{RuO}_{4}$ & 1621 & 1042 & 1071 & 1066 & 1061 & 1405 & 1240 & 1201 & 1114 & 1151 & 1212 & 1242 & 1155 & 1179 & 1163 & 1219 & 1123 & 1175 & 1142 \\
\hline $\mathrm{OsO}_{4}$ & 997 & 783 & 816 & 811 & 803 & 947 & 896 & 862 & 825 & 802 & 852 & 877 & 841 & 844 & 843 & 861 & 827 & 860 & 832 \\
\hline
\end{tabular}

TABLE S58. Statistical evaluation of various computational methods with respect to the experimental findings. MAE, MSD, and STD denote the mean absolute error, mean signed error, and the standard deviation (all in ppm) regarding the latter.

\begin{tabular}{|c|c|c|c|c|c|c|c|c|c|c|c|c|c|c|c|c|c|c|}
\hline & $\mathrm{HF}$ & KT3 & BP86 & PBE & TPSS & BH\&HLYP & B3LYP & PBE0 & TPSSh & LC- $\omega$ PBE & $\omega \mathrm{B} 97 \mathrm{X}-\mathrm{D}$ & CAM-B3LYP & $\mathrm{LH} 07 \mathrm{t}$ & LH12ct & LH14t & LH20t & mPSTS & LHJ14 \\
\hline MAE & 226 & 76 & 50 & 52 & 61 & 100 & 50 & 40 & 38 & 49 & 27 & 32 & 25 & 24 & 26 & 26 & 36 & 27 \\
\hline Max. Deviation & 824 & 224 & 188 & 191 & 210 & 263 & 107 & 141 & 146 & 99 & 70 & 100 & 78 & 56 & 73 & 77 & 139 & 63 \\
\hline MSD & 218 & -76 & -49 & -52 & -61 & 100 & 23 & -9 & -38 & -47 & 0 & 19 & -17 & -10 & -15 & 8 & -36 & 1 \\
\hline STD & 292 & 72 & 67 & 67 & 71 & 97 & 61 & 61 & 53 & 33 & 36 & 40 & 34 & 31 & 35 & 35 & 51 & 35 \\
\hline
\end{tabular}


TABLE S59. Comparison of ${ }^{17} \mathrm{O}$ NMR shielding constants (in ppm) at various levels. LH07, LH12ct, and LH14t refer to the functionals LH07-SVWN, LH12ct-SsirPW92, and LH14t-calPBE, respectively.

\begin{tabular}{lccrrrrrrrr}
\hline & CAM-QTP-00 & CAM-QTP-02 & cTPSS & cTPSSh & cLH07t & cLH12ct & cLH14t & cLH20t & cmPSTS & cLHJ14 \\
\hline $\mathrm{H}_{2} \mathrm{O}$ & 337 & 336 & 321 & 324 & 335 & 340 & 335 & 338 & 323 & 329 \\
$\mathrm{CrO}_{4}^{2-}$ & 573 & -505 & -451 & -473 & -467 & -465 & -469 & -479 & -473 & -478 \\
$\mathrm{MoO}_{4}^{2-}$ & -226 & -212 & -244 & -243 & -220 & -209 & -222 & -217 & -242 & -239 \\
$\mathrm{WO}_{4}^{2-}$ & -105 & -96 & -124 & -121 & -103 & -93 & -106 & -100 & -120 & -122 \\
$\mathrm{MnO}_{4}^{-}$ & -1147 & -980 & -750 & -812 & -834 & -853 & -841 & -884 & -821 & -829 \\
$\mathrm{TcO}_{4}^{-}$ & -503 & -467 & -460 & -471 & -447 & -439 & -453 & -457 & -473 & -468 \\
$\mathrm{ReO}_{4}^{-}$ & -288 & -268 & -283 & -286 & -265 & -255 & -269 & -266 & -286 & -287 \\
$\mathrm{FeO}_{4}$ & -2856 & -2215 & -1192 & -1366 & -1519 & -1647 & -1540 & -1739 & -1402 & -1426 \\
$\mathrm{RuO}_{4}$ & -1058 & -934 & -763 & -813 & -809 & -821 & -821 & -859 & -823 & -818 \\
$\mathrm{OsO}_{4}$ & -587 & -535 & -493 & -511 & -495 & -490 & -502 & -510 & -515 & -515 \\
\hline
\end{tabular}

TABLE S60. Comparison of ${ }^{17} \mathrm{O}$ NMR shifts (in ppm) at various levels. Experimental results are taken from Ref. 102

\begin{tabular}{|c|c|c|c|c|c|c|c|c|c|c|c|}
\hline & CAM-QTP-00 & CAM-QTP-02 & cTPSS & cTPSSh & cLH07t & cLH12ct & cLH14t & cLH20t & cmPSTS & cLHJ14 & Exp. \\
\hline $\mathrm{CrO}_{4}^{2-}$ & 910 & 841 & 772 & 797 & 802 & 804 & 804 & 818 & 796 & 807 & 871 \\
\hline $\mathrm{MoO}_{4}^{2-}$ & 562 & 548 & 565 & 568 & 555 & 548 & 557 & 556 & 565 & 569 & 576 \\
\hline $\mathrm{WO}_{4}^{2-}$ & 4442 & 432 & 45 & 445 & 439 & 433 & 440 & 438 & 443 & 452 & 456 \\
\hline $\mathrm{MnO}_{4}^{-}$ & 1481 & 1316 & 1071 & 1136 & 1169 & 1193 & 1175 & 1223 & 1144 & 1158 & 1255 \\
\hline $\mathrm{TcO}_{4}^{-}$ & 840 & 803 & 780 & 795 & 783 & 779 & 787 & 795 & 796 & 798 & 786 \\
\hline $\mathrm{ReO}_{4}^{-}$ & 625 & 604 & 604 & 610 & 601 & 595 & 604 & 604 & 609 & 616 & 605 \\
\hline $\mathrm{FeO}_{4}^{4}$ & 3193 & 2551 & 1512 & 1690 & 1854 & 1986 & 1875 & 2077 & 1725 & 1755 & - \\
\hline $\mathrm{RuO}_{4}$ & 1394 & 1270 & 1083 & 1137 & 1145 & 1161 & 1155 & 1198 & 1146 & 1147 & 1142 \\
\hline $\mathrm{OsO}_{4}$ & 924 & 871 & 814 & 835 & 830 & 829 & 837 & 848 & 838 & 845 & 832 \\
\hline
\end{tabular}

TABLE S61. Statistical evaluation of various computational methods with respect to the experimental findings. MAE, MSD, and STD denote the mean absolute error, mean signed error, and the standard deviation (all in ppm) regarding the latter.

\begin{tabular}{lccrrrrrrrr}
\hline & CAM-QTP-00 & CAM-QTP-02 & cTPSS & cTPSSh & cLH07t & cLH12ct & cLH14t & cLH20t & cmPSTS & cLHJ14 \\
\hline MAE & 89 & 41 & 49 & 29 & 26 & 27 & 25 & 26 & 29 & 27 \\
Max. Deviation & 252 & 128 & 184 & 119 & 86 & 67 & 80 & 56 & 111 & 97 \\
MSD & 82 & 20 & -49 & -25 & -25 & -23 & -20 & -5 & -23 & -16 \\
STD & 104 & 55 & 64 & 46 & 34 & 29 & 34 & 33 & 45 & 41 \\
\hline
\end{tabular}


2. Optimized Structures at the HF Level

The Cartesian coordinates of the structures are listed in Ångström.

\begin{tabular}{|c|c|c|c|}
\hline 0 & 0.0000000 & 0.0000000 & 0.3748159 \\
\hline $\mathrm{H}$ & -0.7536936 & 0.0000000 & -0.1874085 \\
\hline $\mathrm{H}$ & 0.7536936 & 0.0000000 & -0.1874085 \\
\hline $\mathrm{Cr}$ & 0.0000000 & -0.0000000 & 0.0000000 \\
\hline 0 & -0.9193175 & 0.9193175 & 0.9193175 \\
\hline 0 & 0.9193175 & -0.9193175 & 0.9193175 \\
\hline 0 & -0.9193175 & -0.9193175 & -0.9193175 \\
\hline 0 & 0.9193175 & 0.9193175 & -0.9193175 \\
\hline Mo & 0.0000000 & -0.0000000 & 0.0000000 \\
\hline 0 & 1.0013988 & -1.0013988 & 1.0013988 \\
\hline 0 & -1.0013988 & 1.0013988 & 1.0013988 \\
\hline 0 & 1.0013988 & 1.0013988 & -1.0013988 \\
\hline 0 & -1.0013988 & -1.0013988 & -1.0013988 \\
\hline W & 0.0000000 & -0.0000000 & 0.0000000 \\
\hline 0 & -1.0156131 & 1.0156131 & 1.0156131 \\
\hline 0 & 1.0156131 & -1.0156131 & 1.0156131 \\
\hline 0 & 1.0156131 & 1.0156131 & -1.0156131 \\
\hline 0 & -1.0156131 & -1.0156131 & -1.0156131 \\
\hline Mn & 0.0000114 & 0.0000015 & 0.0000124 \\
\hline 0 & 0.8864082 & -0.8864242 & 0.8864046 \\
\hline 0 & -0.8864169 & 0.8864126 & 0.8863968 \\
\hline 0 & -0.8864055 & -0.8864050 & -0.8864000 \\
\hline 0 & 0.8864028 & 0.8864150 & -0.8864138 \\
\hline Tc & 0.0000366 & -0.0000075 & 0.0000169 \\
\hline 0 & 0.9610003 & -0.9610222 & 0.9610178 \\
\hline 0 & -0.9610097 & 0.9609724 & 0.9609552 \\
\hline 0 & -0.9609985 & -0.9609485 & -0.9609725 \\
\hline 0 & 0.9609712 & 0.9610058 & -0.9610174 \\
\hline $\mathrm{Re}$ & 0.0000346 & -0.0000126 & 0.0000248 \\
\hline 0 & 0.9784937 & -0.9784868 & 0.9784794 \\
\hline 0 & -0.9784793 & 0.9784403 & 0.9783949 \\
\hline 0 & -0.9784476 & -0.9783706 & -0.9784390 \\
\hline 0 & 0.9783986 & 0.9784297 & -0.9784601 \\
\hline $\mathrm{Fe}$ & -0.0000047 & -0.0000129 & -0.0000001 \\
\hline 0 & 0.8718087 & 0.8718041 & 0.8718087 \\
\hline 0 & -0.8717873 & -0.8717906 & 0.8717832 \\
\hline 0 & 0.8717900 & -0.8717983 & -0.8717904 \\
\hline 0 & -0.8718067 & 0.8717976 & -0.8718013 \\
\hline
\end{tabular}




$\begin{array}{lrrr}\mathrm{Ru} & 0.0000000 & 0.0000000 & -0.0000000 \\ 0 & 0.9347827 & 0.9347825 & 0.9347826 \\ 0 & -0.9347825 & -0.9347826 & 0.9347827 \\ 0 & 0.9347824 & -0.9347826 & -0.9347829 \\ 0 & -0.9347827 & 0.9347827 & -0.9347824 \\ & & & \\ 0 \mathrm{~s} & -0.0000000 & 0.0000000 & 0.0000000 \\ 0 & 0.9527198 & -0.9527198 & 0.9527198 \\ 0 & -0.9527198 & 0.9527198 & 0.9527198 \\ 0 & -0.9527198 & -0.9527198 & -0.9527198 \\ 0 & 0.9527198 & 0.9527198 & -0.9527198\end{array}$


3. Optimized Structures at the KT3 Level

The Cartesian coordinates of the structures are listed in Ångström.

\begin{tabular}{|c|c|c|c|}
\hline 0 & 0.0000000 & 0.0000000 & 0.3931854 \\
\hline $\mathrm{H}$ & -0.7508135 & 0.0000000 & -0.1965933 \\
\hline $\mathrm{H}$ & 0.7508135 & 0.0000000 & -0.1965933 \\
\hline $\mathrm{Cr}$ & 0.0000000 & 0.0000000 & 0.0000000 \\
\hline 0 & -0.9486078 & 0.9486078 & 0.9486078 \\
\hline 0 & 0.9486078 & -0.9486078 & 0.9486078 \\
\hline 0 & -0.9486078 & -0.9486078 & -0.9486078 \\
\hline 0 & 0.9486078 & 0.9486078 & -0.9486078 \\
\hline Mo & -0.0000000 & 0.0000000 & -0.0000000 \\
\hline 0 & 1.0207043 & -1.0207043 & 1.0207043 \\
\hline 0 & -1.0207043 & 1.0207043 & 1.0207043 \\
\hline 0 & 1.0207043 & 1.0207043 & -1.0207043 \\
\hline 0 & -1.0207043 & -1.0207043 & -1.0207043 \\
\hline W & 0.0000000 & -0.0000000 & 0.0000000 \\
\hline 0 & -1.0295734 & 1.0295734 & 1.0295734 \\
\hline 0 & 1.0295734 & -1.0295734 & 1.0295734 \\
\hline 0 & 1.0295734 & 1.0295734 & -1.0295734 \\
\hline 0 & -1.0295734 & -1.0295734 & -1.0295734 \\
\hline Mn & 0.0000035 & 0.0000061 & -0.0000047 \\
\hline 0 & 0.9224087 & -0.9224192 & 0.9224132 \\
\hline 0 & -0.9224167 & 0.9224144 & 0.9224178 \\
\hline 0 & -0.9224164 & -0.9224235 & -0.9224092 \\
\hline 0 & 0.9224210 & 0.9224221 & -0.9224171 \\
\hline Tc & 0.0000188 & -0.0000078 & 0.0000131 \\
\hline 0 & 0.9898119 & -0.9898055 & 0.9897867 \\
\hline 0 & -0.9897884 & 0.9897838 & 0.9897281 \\
\hline 0 & -0.9897540 & -0.9897127 & -0.9897675 \\
\hline 0 & 0.9897117 & 0.9897421 & -0.9897604 \\
\hline $\mathrm{Re}$ & 0.0000176 & -0.0000083 & 0.0000112 \\
\hline 0 & 1.0002731 & -1.0002690 & 1.0002585 \\
\hline 0 & -1.0002526 & 1.0002465 & 1.0002025 \\
\hline 0 & -1.0002268 & -1.0001872 & -1.0002374 \\
\hline 0 & 1.0001887 & 1.0002180 & -1.0002348 \\
\hline $\mathrm{Fe}$ & -0.0000000 & 0.0000004 & 0.0000000 \\
\hline 0 & 0.9102624 & 0.9102610 & 0.9102621 \\
\hline 0 & -0.9102605 & -0.9102617 & 0.9102609 \\
\hline 0 & 0.9102600 & -0.9102609 & -0.9102608 \\
\hline 0 & -0.9102619 & 0.9102612 & -0.9102622 \\
\hline
\end{tabular}




$\begin{array}{lrrr}\mathrm{Ru} & 0.0000001 & -0.0000001 & -0.0000000 \\ 0 & 0.9725761 & 0.9725756 & 0.9725760 \\ 0 & -0.9725757 & -0.9725757 & 0.9725759 \\ 0 & 0.9725756 & -0.9725756 & -0.9725762 \\ 0 & -0.9725760 & 0.9725757 & -0.9725756 \\ & & & \\ 0 \mathrm{~s} & 0.0000001 & -0.0000000 & 0.0000000 \\ 0 & 0.9822535 & -0.9822533 & 0.9822534 \\ 0 & -0.9822535 & 0.9822534 & 0.9822534 \\ 0 & -0.9822535 & -0.9822533 & -0.9822534 \\ 0 & 0.9822535 & 0.9822533 & -0.9822534\end{array}$


4. Optimized Structures at the BP86 Level

The Cartesian coordinates of the structures are listed in Ångström.

\begin{tabular}{|c|c|c|c|}
\hline 0 & 0.0000000 & 0.0000000 & 0.3962203 \\
\hline $\mathrm{H}$ & -0.7665456 & 0.0000000 & -0.1981107 \\
\hline $\mathrm{H}$ & 0.7665456 & 0.0000000 & -0.1981107 \\
\hline $\mathrm{Cr}$ & 0.0000000 & 0.0000000 & 0.0000000 \\
\hline 0 & -0.9575618 & 0.9575618 & 0.9575618 \\
\hline 0 & 0.9575618 & -0.9575618 & 0.9575618 \\
\hline 0 & -0.9575618 & -0.9575618 & -0.9575618 \\
\hline 0 & 0.9575618 & 0.9575618 & -0.9575618 \\
\hline Mo & -0.0000000 & 0.0000000 & 0.0000000 \\
\hline 0 & 1.0308536 & -1.0308536 & 1.0308536 \\
\hline 0 & -1.0308536 & 1.0308536 & 1.0308536 \\
\hline 0 & 1.0308536 & 1.0308536 & -1.0308536 \\
\hline 0 & -1.0308536 & -1.0308536 & -1.0308536 \\
\hline W & 0.0000000 & -0.0000000 & -0.0000000 \\
\hline 0 & -1.0387496 & 1.0387496 & 1.0387496 \\
\hline 0 & 1.0387496 & -1.0387496 & 1.0387496 \\
\hline 0 & 1.0387496 & 1.0387496 & -1.0387496 \\
\hline 0 & -1.0387496 & -1.0387496 & -1.0387496 \\
\hline Mn & 0.0000073 & 0.0000021 & 0.0000031 \\
\hline 0 & 0.9304297 & -0.9304434 & 0.9304325 \\
\hline 0 & -0.9304366 & 0.9304359 & 0.9304293 \\
\hline 0 & -0.9304316 & -0.9304352 & -0.9304276 \\
\hline 0 & 0.9304312 & 0.9304406 & -0.9304373 \\
\hline Tc & 0.0000141 & -0.0000098 & 0.0000100 \\
\hline 0 & 0.9991286 & -0.9991152 & 0.9991065 \\
\hline 0 & -0.9991019 & 0.9990971 & 0.9990495 \\
\hline 0 & -0.9990742 & -0.9990307 & -0.9990882 \\
\hline 0 & 0.9990334 & 0.9990587 & -0.9990778 \\
\hline $\mathrm{Re}$ & 0.0000124 & -0.0000143 & 0.0000088 \\
\hline 0 & 1.0090335 & -1.0090154 & 1.0090144 \\
\hline 0 & -1.0090019 & 1.0090030 & 1.0089571 \\
\hline 0 & -1.0089881 & -1.0089443 & -1.0089993 \\
\hline 0 & 1.0089441 & 1.0089709 & -1.0089809 \\
\hline $\mathrm{Fe}$ & 0.0000001 & 0.0000001 & 0.0000001 \\
\hline 0 & 0.9174285 & 0.9174283 & 0.9174284 \\
\hline 0 & -0.9174281 & -0.9174282 & 0.9174280 \\
\hline 0 & 0.9174278 & -0.9174282 & -0.9174286 \\
\hline 0 & -0.9174282 & 0.9174280 & -0.9174279 \\
\hline
\end{tabular}




$\begin{array}{lrrr}\text { Ru } & 0.0000000 & 0.0000000 & -0.0000000 \\ 0 & 0.9812202 & 0.9812200 & 0.9812201 \\ 0 & -0.9812200 & -0.9812202 & 0.9812201 \\ 0 & 0.9812199 & -0.9812201 & -0.9812204 \\ 0 & -0.9812202 & 0.9812203 & -0.9812198 \\ & & & \\ \text { Os } & 0.0000000 & -0.0000001 & -0.0000000 \\ 0 & 0.9907125 & -0.9907125 & 0.9907127 \\ 0 & -0.9907122 & 0.9907124 & 0.9907123 \\ 0 & -0.9907126 & -0.9907124 & -0.9907126 \\ 0 & 0.9907122 & 0.9907126 & -0.9907124\end{array}$




\section{Optimized Structures at the PBE Level}

The Cartesian coordinates of the structures are listed in Ångström.

\begin{tabular}{|c|c|c|c|}
\hline 0 & 0.0000000 & 0.0000000 & 0.3965973 \\
\hline $\mathrm{H}$ & -0.7658845 & 0.0000000 & -0.1982992 \\
\hline $\mathrm{H}$ & 0.7658845 & 0.0000000 & -0.1982992 \\
\hline $\mathrm{Cr}$ & 0.0000000 & 0.0000000 & 0.0000000 \\
\hline 0 & -0.9573950 & 0.9573950 & 0.9573950 \\
\hline 0 & 0.9573950 & -0.9573950 & 0.9573950 \\
\hline 0 & -0.9573950 & -0.9573950 & -0.9573950 \\
\hline 0 & 0.9573950 & 0.9573950 & -0.9573950 \\
\hline Mo & -0.0000000 & 0.0000000 & 0.0000000 \\
\hline 0 & 1.0302238 & -1.0302238 & 1.0302238 \\
\hline 0 & -1.0302238 & 1.0302238 & 1.0302238 \\
\hline 0 & 1.0302238 & 1.0302238 & -1.0302238 \\
\hline 0 & -1.0302238 & -1.0302238 & -1.0302238 \\
\hline W & -0.0000000 & 0.0000000 & 0.0000000 \\
\hline 0 & -1.0384100 & 1.0384100 & 1.0384100 \\
\hline 0 & 1.0384100 & -1.0384100 & 1.0384100 \\
\hline 0 & 1.0384100 & 1.0384100 & -1.0384100 \\
\hline 0 & -1.0384100 & -1.0384100 & -1.0384100 \\
\hline Mn & 0.0000072 & 0.0000022 & 0.0000030 \\
\hline 0 & 0.9301979 & -0.9302103 & 0.9302002 \\
\hline 0 & -0.9302033 & 0.9302040 & 0.9301968 \\
\hline 0 & -0.9301997 & -0.9302033 & -0.9301965 \\
\hline 0 & 0.9301979 & 0.9302073 & -0.9302035 \\
\hline Tc & 0.0000141 & -0.0000099 & 0.0000100 \\
\hline 0 & 0.9983709 & -0.9983574 & 0.9983491 \\
\hline 0 & -0.9983443 & 0.9983393 & 0.9982923 \\
\hline 0 & -0.9983169 & -0.9982734 & -0.9983308 \\
\hline 0 & 0.9982763 & 0.9983014 & -0.9983205 \\
\hline $\mathrm{Re}$ & 0.0000125 & -0.0000144 & 0.0000088 \\
\hline 0 & 1.0084707 & -1.0084527 & 1.0084520 \\
\hline 0 & -1.0084392 & 1.0084404 & 1.0083947 \\
\hline 0 & -1.0084259 & -1.0083820 & -1.0084369 \\
\hline 0 & 1.0083818 & 1.0084088 & -1.0084186 \\
\hline $\mathrm{Fe}$ & -0.0000000 & 0.0000000 & -0.0000000 \\
\hline 0 & 0.9172176 & 0.9172174 & 0.9172174 \\
\hline 0 & -0.9172174 & -0.9172176 & 0.9172175 \\
\hline 0 & 0.9172173 & -0.9172175 & -0.9172177 \\
\hline 0 & -0.9172176 & 0.9172177 & -0.9172173 \\
\hline
\end{tabular}




$\begin{array}{lrrr}\text { Ru } & -0.0000000 & 0.0000000 & 0.0000000 \\ 0 & 0.9804001 & 0.9803997 & 0.9803998 \\ 0 & -0.9803998 & -0.9803999 & 0.9803999 \\ 0 & 0.9803997 & -0.9803998 & -0.9804001 \\ 0 & -0.9804000 & 0.9804000 & -0.9803996 \\ & & & \\ 0 \text { s } & 0.0000000 & -0.0000000 & 0.0000000 \\ 0 & 0.9899725 & -0.9899725 & 0.9899726 \\ 0 & -0.9899725 & 0.9899725 & 0.9899726 \\ 0 & -0.9899725 & -0.9899725 & -0.9899726 \\ 0 & 0.9899725 & 0.9899725 & -0.9899726\end{array}$


6. Optimized Structures at the TPSS Level

The Cartesian coordinates of the structures are listed in Ångström.

\begin{tabular}{|c|c|c|c|}
\hline 0 & 0.0000000 & 0.0000000 & 0.3952585 \\
\hline $\mathrm{H}$ & -0.7652144 & 0.0000000 & -0.1976298 \\
\hline $\mathrm{H}$ & 0.7652144 & 0.0000000 & -0.1976298 \\
\hline $\mathrm{Cr}$ & -0.0000000 & 0.0000000 & 0.0000000 \\
\hline 0 & -0.9565421 & 0.9565421 & 0.9565421 \\
\hline 0 & 0.9565421 & -0.9565421 & 0.9565421 \\
\hline 0 & -0.9565421 & -0.9565421 & -0.9565421 \\
\hline 0 & 0.9565421 & 0.9565421 & -0.9565421 \\
\hline Mo & -0.0000000 & 0.0000000 & 0.0000000 \\
\hline 0 & 1.0302823 & -1.0302823 & 1.0302823 \\
\hline 0 & -1.0302823 & 1.0302823 & 1.0302823 \\
\hline 0 & 1.0302823 & 1.0302823 & -1.0302823 \\
\hline 0 & -1.0302823 & -1.0302823 & -1.0302823 \\
\hline W & 0.0000000 & -0.0000000 & 0.0000000 \\
\hline 0 & -1.0374341 & 1.0374341 & 1.0374341 \\
\hline 0 & 1.0374341 & -1.0374341 & 1.0374341 \\
\hline 0 & 1.0374341 & 1.0374341 & -1.0374341 \\
\hline 0 & -1.0374341 & -1.0374341 & -1.0374341 \\
\hline Mn & 0.0000078 & 0.0000023 & 0.0000031 \\
\hline 0 & 0.9291247 & -0.9291376 & 0.9291272 \\
\hline 0 & -0.9291306 & 0.9291306 & 0.9291234 \\
\hline 0 & -0.9291267 & -0.9291298 & -0.9291229 \\
\hline 0 & 0.9291248 & 0.9291345 & -0.9291308 \\
\hline Tc & 0.0000157 & -0.0000106 & 0.0000110 \\
\hline 0 & 0.9983398 & -0.9983263 & 0.9983179 \\
\hline 0 & -0.9983128 & 0.9983062 & 0.9982575 \\
\hline 0 & -0.9982844 & -0.9982377 & -0.9982978 \\
\hline 0 & 0.9982417 & 0.9982683 & -0.9982886 \\
\hline $\mathrm{Re}$ & 0.0000139 & -0.0000152 & 0.0000093 \\
\hline 0 & 1.0075505 & -1.0075323 & 1.0075320 \\
\hline 0 & -1.0075183 & 1.0075184 & 1.0074713 \\
\hline 0 & -1.0075049 & -1.0074582 & -1.0075151 \\
\hline 0 & 1.0074588 & 1.0074874 & -1.0074975 \\
\hline $\mathrm{Fe}$ & 0.0000000 & 0.0000000 & 0.0000000 \\
\hline 0 & 0.9157155 & 0.9157152 & 0.9157153 \\
\hline 0 & -0.9157152 & -0.9157154 & 0.9157153 \\
\hline 0 & 0.9157150 & -0.9157151 & -0.9157155 \\
\hline 0 & -0.9157154 & 0.9157153 & -0.9157151 \\
\hline
\end{tabular}




$\begin{array}{lrrr}\text { Ru } & -0.0000000 & -0.0000000 & -0.0000000 \\ 0 & 0.9802171 & 0.9802168 & 0.9802168 \\ 0 & -0.9802168 & -0.9802170 & 0.9802169 \\ 0 & 0.9802168 & -0.9802168 & -0.9802171 \\ 0 & -0.9802171 & 0.9802171 & -0.9802166 \\ & & & \\ 0 s & 0.0000000 & -0.0000000 & -0.0000000 \\ 0 & 0.9890603 & -0.9890603 & 0.9890603 \\ 0 & -0.9890603 & 0.9890603 & 0.9890604 \\ 0 & -0.9890603 & -0.9890603 & -0.9890603 \\ 0 & 0.9890603 & 0.9890603 & -0.9890603\end{array}$


7. Optimized Structures at the BH\&HLYP Level

The Cartesian coordinates of the structures are listed in Ångström.

\begin{tabular}{|c|c|c|c|}
\hline 0 & 0.0000000 & 0.0000000 & 0.3810042 \\
\hline $\mathrm{H}$ & -0.7584633 & 0.0000000 & -0.1905026 \\
\hline $\mathrm{H}$ & 0.7584633 & -0.0000000 & -0.1905026 \\
\hline $\mathrm{Cr}$ & 0.0000000 & -0.0000000 & 0.0000000 \\
\hline 0 & -0.9318374 & 0.9318374 & 0.9318374 \\
\hline 0 & 0.9318374 & -0.9318374 & 0.9318374 \\
\hline 0 & -0.9318374 & -0.9318374 & -0.9318374 \\
\hline 0 & 0.9318374 & 0.9318374 & -0.9318374 \\
\hline Mo & 0.0000000 & 0.0000000 & 0.0000000 \\
\hline 0 & 1.0105129 & -1.0105129 & 1.0105129 \\
\hline 0 & -1.0105129 & 1.0105129 & 1.0105129 \\
\hline 0 & 1.0105129 & 1.0105129 & -1.0105129 \\
\hline 0 & -1.0105129 & -1.0105129 & -1.0105129 \\
\hline W & -0.0000000 & 0.0000000 & 0.0000000 \\
\hline 0 & -1.0220443 & 1.0220443 & 1.0220443 \\
\hline 0 & 1.0220443 & -1.0220443 & 1.0220443 \\
\hline 0 & 1.0220443 & 1.0220443 & -1.0220443 \\
\hline 0 & -1.0220443 & -1.0220443 & -1.0220443 \\
\hline Mn & 0.0000053 & 0.0000068 & -0.0000009 \\
\hline 0 & 0.9013728 & -0.9013859 & 0.9013779 \\
\hline 0 & -0.9013836 & 0.9013785 & 0.9013821 \\
\hline 0 & -0.9013807 & -0.9013872 & -0.9013734 \\
\hline 0 & 0.9013862 & 0.9013878 & -0.9013856 \\
\hline Tc & 0.0000280 & -0.0000080 & 0.0000113 \\
\hline 0 & 0.9745821 & -0.9745929 & 0.9745955 \\
\hline 0 & -0.9745878 & 0.9745546 & 0.9745446 \\
\hline 0 & -0.9745820 & -0.9745365 & -0.9745585 \\
\hline 0 & 0.9745597 & 0.9745829 & -0.9745929 \\
\hline $\mathrm{Re}$ & 0.0000291 & -0.0000148 & 0.0000237 \\
\hline 0 & 0.9888667 & -0.9888662 & 0.9888467 \\
\hline 0 & -0.9888472 & 0.9888306 & 0.9887696 \\
\hline 0 & -0.9888134 & -0.9887553 & -0.9888155 \\
\hline 0 & 0.9887648 & 0.9888058 & -0.9888246 \\
\hline $\mathrm{Fe}$ & 0.0000001 & 0.0000001 & -0.0000000 \\
\hline 0 & 0.8857215 & 0.8857212 & 0.8857213 \\
\hline 0 & -0.8857212 & -0.8857214 & 0.8857213 \\
\hline 0 & 0.8857211 & -0.8857213 & -0.8857215 \\
\hline 0 & -0.8857215 & 0.8857214 & -0.8857211 \\
\hline
\end{tabular}




$\begin{array}{lrrr}\mathrm{Ru} & 0.0000006 & -0.0000006 & -0.0000008 \\ 0 & 0.9525376 & 0.9525396 & 0.9525422 \\ 0 & -0.9525382 & -0.9525389 & 0.9525422 \\ 0 & 0.9525387 & -0.9525403 & -0.9525427 \\ 0 & -0.9525386 & 0.9525401 & -0.9525409 \\ & & & \\ & & & \\ \text { Os } & 0.0000010 & -0.0000000 & -0.0000005 \\ 0 & 0.9668497 & -0.9668519 & 0.9668510 \\ 0 & -0.9668502 & 0.9668507 & 0.9668499 \\ 0 & -0.9668508 & -0.9668513 & -0.9668496 \\ 0 & 0.9668503 & 0.9668525 & -0.9668508\end{array}$


8. Optimized Structures at the B3LYP Level

The Cartesian coordinates of the structures are listed in Ångström.

\begin{tabular}{|c|c|c|c|}
\hline 0 & 0.0000000 & 0.0000000 & 0.3889942 \\
\hline $\mathrm{H}$ & -0.7642840 & 0.0000000 & -0.1944977 \\
\hline $\mathrm{H}$ & 0.7642840 & -0.0000000 & -0.1944977 \\
\hline $\mathrm{Cr}$ & 0.0000000 & 0.0000000 & 0.0000000 \\
\hline 0 & -0.9572515 & 0.9572515 & 0.9572515 \\
\hline 0 & 0.9572515 & -0.9572515 & 0.9572515 \\
\hline 0 & -0.9572515 & -0.9572515 & -0.9572515 \\
\hline 0 & 0.9572515 & 0.9572515 & -0.9572515 \\
\hline Mo & -0.0000000 & 0.0000000 & 0.0000000 \\
\hline 0 & 1.0242859 & -1.0242859 & 1.0242859 \\
\hline 0 & -1.0242859 & 1.0242859 & 1.0242859 \\
\hline 0 & 1.0242859 & 1.0242859 & -1.0242859 \\
\hline 0 & -1.0242859 & -1.0242859 & -1.0242859 \\
\hline W & 0.0000000 & 0.0000000 & 0.0000000 \\
\hline 0 & -1.0336129 & 1.0336129 & 1.0336129 \\
\hline 0 & 1.0336129 & -1.0336129 & 1.0336129 \\
\hline 0 & 1.0336129 & 1.0336129 & -1.0336129 \\
\hline 0 & -1.0336129 & -1.0336129 & -1.0336129 \\
\hline Mn & 0.0000033 & 0.0000064 & -0.0000058 \\
\hline 0 & 0.9202003 & -0.9202103 & 0.9202047 \\
\hline 0 & -0.9202086 & 0.9202047 & 0.9202090 \\
\hline 0 & -0.9202088 & -0.9202147 & -0.9201994 \\
\hline 0 & 0.9202139 & 0.9202139 & -0.9202085 \\
\hline Tc & 0.0000129 & -0.0000096 & 0.0000049 \\
\hline 0 & 0.9908327 & -0.9908174 & 0.9908159 \\
\hline 0 & -0.9908066 & 0.9907998 & 0.9907608 \\
\hline 0 & -0.9907854 & -0.9907412 & -0.9907956 \\
\hline 0 & 0.9907464 & 0.9907685 & -0.9907859 \\
\hline $\mathrm{Re}$ & 0.0000193 & -0.0000080 & 0.0000110 \\
\hline 0 & 1.0023372 & -1.0023361 & 1.0023253 \\
\hline 0 & -1.0023206 & 1.0023118 & 1.0022709 \\
\hline 0 & -1.0022960 & -1.0022570 & -1.0023025 \\
\hline 0 & 1.0022602 & 1.0022893 & -1.0023046 \\
\hline $\mathrm{Fe}$ & 0.0000000 & -0.0000000 & -0.0000000 \\
\hline 0 & 0.9063385 & 0.9063382 & 0.9063384 \\
\hline 0 & -0.9063382 & -0.9063384 & 0.9063384 \\
\hline 0 & 0.9063381 & -0.9063383 & -0.9063386 \\
\hline 0 & -0.9063384 & 0.9063385 & -0.9063381 \\
\hline
\end{tabular}




$\begin{array}{lrrr}\mathrm{Ru} & 0.0000001 & -0.0000001 & -0.0000001 \\ \mathrm{O} & 0.9713539 & 0.9713541 & 0.9713546 \\ 0 & -0.9713538 & -0.9713541 & 0.9713547 \\ 0 & 0.9713539 & -0.9713542 & -0.9713549 \\ 0 & -0.9713541 & 0.9713544 & -0.9713542 \\ & & & \\ & & & \\ \text { Os } & -0.0000000 & 0.0000000 & -0.0000000 \\ 0 & 0.9825521 & -0.9825521 & 0.9825522 \\ 0 & -0.9825521 & 0.9825521 & 0.9825522 \\ 0 & -0.9825521 & -0.9825521 & -0.9825522 \\ 0 & 0.9825521 & 0.9825521 & -0.9825522\end{array}$


9. Optimized Structures at the PBEO Level

The Cartesian coordinates of the structures are listed in Ångström.

\begin{tabular}{|c|c|c|c|}
\hline 0 & 0.0000000 & 0.0000000 & 0.3884207 \\
\hline $\mathrm{H}$ & -0.7607186 & 0.0000000 & -0.1942109 \\
\hline $\mathrm{H}$ & 0.7607186 & 0.0000000 & -0.1942109 \\
\hline $\mathrm{Cr}$ & -0.0000000 & 0.0000000 & 0.0000000 \\
\hline 0 & -0.9403213 & 0.9403213 & 0.9403213 \\
\hline 0 & 0.9403213 & -0.9403213 & 0.9403213 \\
\hline 0 & -0.9403213 & -0.9403213 & -0.9403213 \\
\hline 0 & 0.9403213 & 0.9403213 & -0.9403213 \\
\hline Mo & -0.0000000 & 0.0000000 & -0.0000000 \\
\hline 0 & 1.0161021 & -1.0161021 & 1.0161021 \\
\hline 0 & -1.0161021 & 1.0161021 & 1.0161021 \\
\hline 0 & 1.0161021 & 1.0161021 & -1.0161021 \\
\hline 0 & -1.0161021 & -1.0161021 & -1.0161021 \\
\hline W & -0.0000000 & 0.0000000 & 0.0000000 \\
\hline 0 & -1.0263919 & 1.0263919 & 1.0263919 \\
\hline 0 & 1.0263919 & -1.0263919 & 1.0263919 \\
\hline 0 & 1.0263919 & 1.0263919 & -1.0263919 \\
\hline 0 & -1.0263919 & -1.0263919 & -1.0263919 \\
\hline Mn & 0.0000032 & 0.0000076 & -0.0000030 \\
\hline 0 & 0.9114569 & -0.9114689 & 0.9114616 \\
\hline 0 & -0.9114663 & 0.9114632 & 0.9114675 \\
\hline 0 & -0.9114641 & -0.9114726 & -0.9114582 \\
\hline 0 & 0.9114703 & 0.9114706 & -0.9114678 \\
\hline Tc & 0.0000245 & -0.0000079 & 0.0000172 \\
\hline 0 & 0.9821623 & -0.9821561 & 0.9821426 \\
\hline 0 & -0.9821437 & 0.9821241 & 0.9820756 \\
\hline 0 & -0.9821111 & -0.9820567 & -0.9821150 \\
\hline 0 & 0.9820680 & 0.9820966 & -0.9821205 \\
\hline $\mathrm{Re}$ & 0.0000179 & -0.0000141 & 0.0000136 \\
\hline 0 & 0.9948875 & -0.9948726 & 0.9948692 \\
\hline 0 & -0.9948605 & 0.9948505 & 0.9948041 \\
\hline 0 & -0.9948378 & -0.9947848 & -0.9948468 \\
\hline 0 & 0.9947929 & 0.9948210 & -0.9948401 \\
\hline $\mathrm{Fe}$ & -0.0000000 & 0.0000000 & 0.0000000 \\
\hline 0 & 0.8970487 & 0.8970484 & 0.8970484 \\
\hline 0 & -0.8970484 & -0.8970486 & 0.8970484 \\
\hline 0 & 0.8970483 & -0.8970484 & -0.8970486 \\
\hline 0 & -0.8970486 & 0.8970486 & -0.8970482 \\
\hline
\end{tabular}




$\begin{array}{lrrr}\mathrm{Ru} & 0.0000000 & -0.0000000 & -0.0000000 \\ 0 & 0.9619180 & 0.9619177 & 0.9619180 \\ 0 & -0.9619176 & -0.9619179 & 0.9619180 \\ 0 & 0.9619176 & -0.9619179 & -0.9619182 \\ 0 & -0.9619180 & 0.9619180 & -0.9619177 \\ & & & \\ & & & \\ \text { Os } & -0.0000000 & 0.0000000 & 0.0000000 \\ 0 & 0.9744750 & -0.9744749 & 0.9744750 \\ 0 & -0.9744750 & 0.9744750 & 0.9744751 \\ 0 & -0.9744750 & -0.9744750 & -0.9744751 \\ 0 & 0.9744750 & 0.9744749 & -0.9744750\end{array}$


10. Optimized Structures at the TPSSh Level

The Cartesian coordinates of the structures are listed in Ångström.

\begin{tabular}{|c|c|c|c|}
\hline 0 & 0.0000000 & 0.0000000 & 0.3920206 \\
\hline $\mathrm{H}$ & -0.7631190 & 0.0000000 & -0.1960109 \\
\hline $\mathrm{H}$ & 0.7631190 & 0.0000000 & -0.1960109 \\
\hline $\mathrm{Cr}$ & -0.0000000 & 0.0000000 & 0.0000000 \\
\hline 0 & -0.9496084 & 0.9496084 & 0.9496084 \\
\hline 0 & 0.9496084 & -0.9496084 & 0.9496084 \\
\hline 0 & -0.9496084 & -0.9496084 & -0.9496084 \\
\hline 0 & 0.9496084 & 0.9496084 & -0.9496084 \\
\hline Mo & -0.0000000 & 0.0000000 & 0.0000000 \\
\hline 0 & 1.0244137 & -1.0244137 & 1.0244137 \\
\hline 0 & -1.0244137 & 1.0244137 & 1.0244137 \\
\hline 0 & 1.0244137 & 1.0244137 & -1.0244137 \\
\hline 0 & -1.0244137 & -1.0244137 & -1.0244137 \\
\hline W & 0.0000000 & -0.0000000 & 0.0000000 \\
\hline 0 & -1.0326034 & 1.0326034 & 1.0326034 \\
\hline 0 & 1.0326034 & -1.0326034 & 1.0326034 \\
\hline 0 & 1.0326034 & 1.0326034 & -1.0326034 \\
\hline 0 & -1.0326034 & -1.0326034 & -1.0326034 \\
\hline Mn & 0.0000032 & 0.0000063 & -0.0000057 \\
\hline 0 & 0.9215319 & -0.9215424 & 0.9215364 \\
\hline 0 & -0.9215403 & 0.9215369 & 0.9215408 \\
\hline 0 & -0.9215403 & -0.9215469 & -0.9215312 \\
\hline 0 & 0.9215455 & 0.9215460 & -0.9215403 \\
\hline Tc & 0.0000141 & -0.0000106 & 0.0000073 \\
\hline 0 & 0.9915919 & -0.9915765 & 0.9915724 \\
\hline 0 & -0.9915638 & 0.9915579 & 0.9915132 \\
\hline 0 & -0.9915401 & -0.9914937 & -0.9915519 \\
\hline 0 & 0.9914979 & 0.9915229 & -0.9915410 \\
\hline $\mathrm{Re}$ & 0.0000201 & -0.0000079 & 0.0000121 \\
\hline 0 & 1.0020032 & -1.0020033 & 1.0019895 \\
\hline 0 & -1.0019857 & 1.0019780 & 1.0019328 \\
\hline 0 & -1.0019582 & -1.0019190 & -1.0019664 \\
\hline 0 & 1.0019206 & 1.0019522 & -1.0019681 \\
\hline $\mathrm{Fe}$ & 0.0000000 & 0.0000000 & 0.0000000 \\
\hline 0 & 0.9075946 & 0.9075943 & 0.9075944 \\
\hline 0 & -0.9075943 & -0.9075944 & 0.9075944 \\
\hline 0 & 0.9075942 & -0.9075943 & -0.9075946 \\
\hline 0 & -0.9075945 & 0.9075945 & -0.9075942 \\
\hline
\end{tabular}




$\begin{array}{lrrr}\mathrm{Ru} & 0.0000000 & -0.0000000 & 0.0000000 \\ \mathrm{O} & 0.9725430 & 0.9725427 & 0.9725428 \\ 0 & -0.9725427 & -0.9725429 & 0.9725429 \\ 0 & 0.9725427 & -0.9725428 & -0.9725431 \\ 0 & -0.9725430 & 0.9725430 & -0.9725426 \\ & & & \\ & & & \\ \text { Os } & -0.0000000 & 0.0000000 & 0.0000000 \\ 0 & 0.9827161 & -0.9827161 & 0.9827161 \\ 0 & -0.9827161 & 0.9827161 & 0.9827161 \\ 0 & -0.9827161 & -0.9827161 & -0.9827161 \\ 0 & 0.9827161 & 0.9827161 & -0.9827161\end{array}$


11. Optimized Structures at the LC- $\omega P B E$ Level

The Cartesian coordinates of the structures are listed in Ångström.

\begin{tabular}{|c|c|c|c|}
\hline 0 & 0.0000000 & 0.0000000 & 0.3855761 \\
\hline $\mathrm{H}$ & -0.7641972 & 0.0000000 & -0.1927886 \\
\hline $\mathrm{H}$ & 0.7641972 & 0.0000000 & -0.1927886 \\
\hline $\mathrm{Cr}$ & 0.0000000 & 0.0000000 & 0.0000000 \\
\hline 0 & -0.9346069 & 0.9346069 & 0.9346069 \\
\hline 0 & 0.9346069 & -0.9346069 & 0.9346069 \\
\hline 0 & -0.9346069 & -0.9346069 & -0.9346069 \\
\hline 0 & 0.9346069 & 0.9346069 & -0.9346069 \\
\hline Mo & -0.0000000 & 0.0000000 & 0.0000000 \\
\hline 0 & 1.0103903 & -1.0103903 & 1.0103903 \\
\hline 0 & -1.0103903 & 1.0103903 & 1.0103903 \\
\hline 0 & 1.0103903 & 1.0103903 & -1.0103903 \\
\hline 0 & -1.0103903 & -1.0103903 & -1.0103903 \\
\hline W & -0.0000000 & 0.0000000 & 0.0000000 \\
\hline 0 & -1.0222343 & 1.0222343 & 1.0222343 \\
\hline 0 & 1.0222343 & -1.0222343 & 1.0222343 \\
\hline 0 & 1.0222343 & 1.0222343 & -1.0222343 \\
\hline 0 & -1.0222343 & -1.0222343 & -1.0222343 \\
\hline Mn & 0.0000049 & 0.0000074 & -0.0000036 \\
\hline 0 & 0.9049185 & -0.9049366 & 0.9049270 \\
\hline 0 & -0.9049278 & 0.9049279 & 0.9049288 \\
\hline 0 & -0.9049281 & -0.9049387 & -0.9049202 \\
\hline 0 & 0.9049324 & 0.9049400 & -0.9049320 \\
\hline Tc & 0.0000272 & -0.0000073 & 0.0000114 \\
\hline 0 & 0.9750072 & -0.9750201 & 0.9750198 \\
\hline 0 & -0.9750132 & 0.9749828 & 0.9749711 \\
\hline 0 & -0.9750065 & -0.9749650 & -0.9749843 \\
\hline 0 & 0.9749853 & 0.9750095 & -0.9750180 \\
\hline $\mathrm{Re}$ & 0.0000279 & -0.0000139 & 0.0000241 \\
\hline 0 & 0.9895193 & -0.9895194 & 0.9894976 \\
\hline 0 & -0.9894994 & 0.9894852 & 0.9894225 \\
\hline 0 & -0.9894630 & -0.9894080 & -0.9894684 \\
\hline 0 & 0.9894152 & 0.9894561 & -0.9894757 \\
\hline $\mathrm{Fe}$ & 0.0000001 & 0.0000003 & -0.0000000 \\
\hline 0 & 0.8889311 & 0.8889310 & 0.8889317 \\
\hline 0 & -0.8889305 & -0.8889312 & 0.8889313 \\
\hline 0 & 0.8889304 & -0.8889313 & -0.8889316 \\
\hline 0 & -0.8889311 & 0.8889312 & -0.8889314 \\
\hline
\end{tabular}




$\begin{array}{lrrr}\mathrm{Ru} & 0.0000001 & 0.0000000 & -0.0000001 \\ 0 & 0.9529860 & 0.9529860 & 0.9529864 \\ 0 & -0.9529856 & -0.9529861 & 0.9529863 \\ 0 & 0.9529856 & -0.9529862 & -0.9529866 \\ 0 & -0.9529861 & 0.9529863 & -0.9529861 \\ & & & \\ & & & \\ \text { Os } & 0.0000002 & -0.0000001 & -0.0000001 \\ 0 & 0.9675713 & -0.9675717 & 0.9675716 \\ 0 & -0.9675713 & 0.9675715 & 0.9675713 \\ 0 & -0.9675715 & -0.9675716 & -0.9675713 \\ 0 & 0.9675713 & 0.9675718 & -0.9675714\end{array}$


12. Optimized Structures at the $\omega B 97 X-D$ Level

The Cartesian coordinates of the structures are listed in Ångström.

\begin{tabular}{|c|c|c|c|}
\hline 0 & 0.0000000 & 0.0000000 & 0.3862056 \\
\hline $\mathrm{H}$ & -0.7606126 & 0.0000000 & -0.1931033 \\
\hline $\mathrm{H}$ & 0.7606126 & 0.0000000 & -0.1931033 \\
\hline $\mathrm{Cr}$ & -0.0000000 & -0.0000000 & 0.0000000 \\
\hline 0 & -0.9403723 & 0.9403723 & 0.9403723 \\
\hline 0 & 0.9403723 & -0.9403723 & 0.9403723 \\
\hline 0 & -0.9403723 & -0.9403723 & -0.9403723 \\
\hline 0 & 0.9403723 & 0.9403723 & -0.9403723 \\
\hline Mo & 0.0000000 & -0.0000000 & 0.0000000 \\
\hline 0 & 1.0154548 & -1.0154548 & 1.0154548 \\
\hline 0 & -1.0154548 & 1.0154548 & 1.0154548 \\
\hline 0 & 1.0154548 & 1.0154548 & -1.0154548 \\
\hline 0 & -1.0154548 & -1.0154548 & -1.0154548 \\
\hline W & -0.0000000 & 0.0000000 & 0.0000000 \\
\hline 0 & -1.0266089 & 1.0266089 & 1.0266089 \\
\hline 0 & 1.0266089 & -1.0266089 & 1.0266089 \\
\hline 0 & 1.0266089 & 1.0266089 & -1.0266089 \\
\hline 0 & -1.0266089 & -1.0266089 & -1.0266089 \\
\hline Mn & 0.0000050 & 0.0000082 & -0.0000039 \\
\hline 0 & 0.9106120 & -0.9106285 & 0.9106188 \\
\hline 0 & -0.9106228 & 0.9106203 & 0.9106231 \\
\hline 0 & -0.9106211 & -0.9106311 & -0.9106127 \\
\hline 0 & 0.9106270 & 0.9106310 & -0.9106252 \\
\hline Tc & 0.0000244 & -0.0000064 & 0.0000148 \\
\hline 0 & 0.9810167 & -0.9810130 & 0.9809993 \\
\hline 0 & -0.9809997 & 0.9809804 & 0.9809341 \\
\hline 0 & -0.9809678 & -0.9809161 & -0.9809706 \\
\hline 0 & 0.9809264 & 0.9809552 & -0.9809776 \\
\hline $\mathrm{Re}$ & 0.0000169 & -0.0000132 & 0.0000110 \\
\hline 0 & 0.9943415 & -0.9943261 & 0.9943248 \\
\hline 0 & -0.9943157 & 0.9943048 & 0.9942626 \\
\hline 0 & -0.9942953 & -0.9942436 & -0.9943024 \\
\hline 0 & 0.9942526 & 0.9942780 & -0.9942961 \\
\hline $\mathrm{Fe}$ & 0.0000000 & 0.0000000 & -0.0000000 \\
\hline 0 & 0.8957101 & 0.8957098 & 0.8957101 \\
\hline 0 & -0.8957098 & -0.8957100 & 0.8957101 \\
\hline 0 & 0.8957097 & -0.8957099 & -0.8957103 \\
\hline 0 & -0.8957101 & 0.8957101 & -0.8957098 \\
\hline
\end{tabular}




$\begin{array}{lrrr}\mathrm{Ru} & 0.0000001 & -0.0000000 & -0.0000001 \\ \mathrm{O} & 0.9598447 & 0.9598446 & 0.9598450 \\ 0 & -0.9598444 & -0.9598447 & 0.9598450 \\ 0 & 0.9598444 & -0.9598447 & -0.9598452 \\ 0 & -0.9598447 & 0.9598449 & -0.9598447 \\ & & & \\ & & & \\ \text { Os } & 0.0000011 & -0.0000002 & -0.0000006 \\ 0 & 0.9732295 & -0.9732322 & 0.9732312 \\ 0 & -0.9732297 & 0.9732309 & 0.9732294 \\ 0 & -0.9732308 & -0.9732316 & -0.9732295 \\ 0 & 0.9732299 & 0.9732331 & -0.9732305\end{array}$


13. Optimized Structures at the CAM-B3LYP Level

The Cartesian coordinates of the structures are listed in Ångström.

\begin{tabular}{|c|c|c|c|}
\hline 0 & 0.0000000 & 0.0000000 & 0.3861673 \\
\hline $\mathrm{H}$ & -0.7650684 & 0.0000000 & -0.1930842 \\
\hline $\mathrm{H}$ & 0.7650684 & -0.0000000 & -0.1930842 \\
\hline $\mathrm{Cr}$ & -0.0000000 & 0.0000000 & 0.0000000 \\
\hline 0 & -0.9397588 & 0.9397588 & 0.9397588 \\
\hline 0 & 0.9397588 & -0.9397588 & 0.9397588 \\
\hline 0 & -0.9397588 & -0.9397588 & -0.9397588 \\
\hline 0 & 0.9397588 & 0.9397588 & -0.9397588 \\
\hline Mo & -0.0000000 & 0.0000000 & 0.0000000 \\
\hline 0 & 1.0164364 & -1.0164364 & 1.0164364 \\
\hline 0 & -1.0164364 & 1.0164364 & 1.0164364 \\
\hline 0 & 1.0164364 & 1.0164364 & -1.0164364 \\
\hline 0 & -1.0164364 & -1.0164364 & -1.0164364 \\
\hline W & -0.0000000 & 0.0000000 & 0.0000000 \\
\hline 0 & -1.0267875 & 1.0267875 & 1.0267875 \\
\hline 0 & 1.0267875 & -1.0267875 & 1.0267875 \\
\hline 0 & 1.0267875 & 1.0267875 & -1.0267875 \\
\hline 0 & -1.0267875 & -1.0267875 & -1.0267875 \\
\hline $\mathrm{Mn}$ & 0.0000031 & 0.0000078 & -0.0000035 \\
\hline 0 & 0.9105872 & -0.9105989 & 0.9105920 \\
\hline 0 & -0.9105967 & 0.9105931 & 0.9105981 \\
\hline 0 & -0.9105948 & -0.9106030 & -0.9105884 \\
\hline 0 & 0.9106012 & 0.9106010 & -0.9105982 \\
\hline Tc & 0.0000250 & -0.0000080 & 0.0000173 \\
\hline 0 & 0.9819731 & -0.9819677 & 0.9819554 \\
\hline 0 & -0.9819561 & 0.9819349 & 0.9818888 \\
\hline 0 & -0.9819246 & -0.9818699 & -0.9819269 \\
\hline 0 & 0.9818826 & 0.9819107 & -0.9819346 \\
\hline $\operatorname{Re}$ & 0.0000177 & -0.0000145 & 0.0000129 \\
\hline 0 & 0.9949281 & -0.9949124 & 0.9949117 \\
\hline 0 & -0.9949023 & 0.9948905 & 0.9948480 \\
\hline 0 & -0.9948823 & -0.9948286 & -0.9948891 \\
\hline 0 & 0.9948388 & 0.9948649 & -0.9948835 \\
\hline $\mathrm{Fe}$ & -0.0000000 & 0.0000000 & 0.0000000 \\
\hline 0 & 0.8955857 & 0.8955854 & 0.8955855 \\
\hline 0 & -0.8955854 & -0.8955856 & 0.8955855 \\
\hline 0 & 0.8955853 & -0.8955854 & -0.8955857 \\
\hline 0 & -0.8955856 & 0.8955856 & -0.8955853 \\
\hline
\end{tabular}




$\begin{array}{lrrr}\mathrm{Ru} & 0.0000003 & -0.0000004 & -0.0000007 \\ \mathrm{O} & 0.9610724 & 0.9610748 & 0.9610775 \\ 0 & -0.9610728 & -0.9610744 & 0.9610778 \\ 0 & 0.9610734 & -0.9610754 & -0.9610780 \\ 0 & -0.9610733 & 0.9610755 & -0.9610766 \\ & & & \\ \text { Os } & 0.0000000 & -0.0000000 & -0.0000000 \\ 0 & 0.9740766 & -0.9740766 & 0.9740766 \\ 0 & -0.9740766 & 0.9740766 & 0.9740766 \\ 0 & -0.9740766 & -0.9740766 & -0.9740766 \\ 0 & 0.9740766 & 0.9740766 & -0.9740766\end{array}$


14. Optimized Structures at the CAM-QTP-00 Level

The Cartesian coordinates of the structures are listed in Ångström.

\begin{tabular}{|c|c|c|c|}
\hline 0 & 0.0000000 & 0.0000000 & 0.3781668 \\
\hline $\mathrm{H}$ & -0.7579821 & 0.0000000 & -0.1890839 \\
\hline $\mathrm{H}$ & 0.7579821 & 0.0000000 & -0.1890839 \\
\hline $\mathrm{Cr}$ & 0.0000000 & 0.0000000 & -0.0000000 \\
\hline 0 & -0.9239567 & 0.9239567 & 0.9239567 \\
\hline 0 & 0.9239567 & -0.9239567 & 0.9239567 \\
\hline 0 & -0.9239567 & -0.9239567 & -0.9239567 \\
\hline 0 & 0.9239567 & 0.9239567 & -0.9239567 \\
\hline Mo & -0.0000000 & -0.0000000 & -0.0000000 \\
\hline 0 & 1.0035180 & -1.0035180 & 1.0035180 \\
\hline 0 & -1.0035180 & 1.0035180 & 1.0035180 \\
\hline 0 & 1.0035180 & 1.0035180 & -1.0035180 \\
\hline 0 & -1.0035180 & -1.0035180 & -1.0035180 \\
\hline W & 0.0000000 & -0.0000000 & -0.0000000 \\
\hline 0 & -1.0158681 & 1.0158681 & 1.0158681 \\
\hline 0 & 1.0158681 & -1.0158681 & 1.0158681 \\
\hline 0 & 1.0158681 & 1.0158681 & -1.0158681 \\
\hline 0 & -1.0158681 & -1.0158681 & -1.0158681 \\
\hline Mn & 0.0000108 & 0.0000011 & 0.0000108 \\
\hline 0 & 0.8928231 & -0.8928391 & 0.8928214 \\
\hline 0 & -0.8928296 & 0.8928284 & 0.8928124 \\
\hline 0 & -0.8928216 & -0.8928221 & -0.8928167 \\
\hline 0 & 0.8928173 & 0.8928316 & -0.8928279 \\
\hline Tc & 0.0000212 & -0.0000039 & 0.0000070 \\
\hline 0 & 0.9667425 & -0.9667460 & 0.9667571 \\
\hline 0 & -0.9667479 & 0.9667160 & 0.9667197 \\
\hline 0 & -0.9667450 & -0.9667060 & -0.9667284 \\
\hline 0 & 0.9667292 & 0.9667398 & -0.9667554 \\
\hline $\mathrm{Re}$ & 0.0000328 & -0.0000120 & 0.0000248 \\
\hline 0 & 0.9821311 & -0.9821272 & 0.9821163 \\
\hline 0 & -0.9821168 & 0.9820847 & 0.9820370 \\
\hline 0 & -0.9820843 & -0.9820155 & -0.9820802 \\
\hline 0 & 0.9820372 & 0.9820700 & -0.9820980 \\
\hline $\mathrm{Fe}$ & -0.0000001 & -0.0000000 & 0.0000000 \\
\hline 0 & 0.8762222 & 0.8762222 & 0.8762225 \\
\hline 0 & -0.8762219 & -0.8762223 & 0.8762227 \\
\hline 0 & 0.8762220 & -0.8762223 & -0.8762229 \\
\hline 0 & -0.8762221 & 0.8762225 & -0.8762224 \\
\hline
\end{tabular}




$\begin{array}{lrrr}\text { Ru } & 0.0000000 & 0.0000000 & -0.0000000 \\ 0 & 0.9435437 & 0.9435435 & 0.9435439 \\ 0 & -0.9435433 & -0.9435437 & 0.9435440 \\ 0 & 0.9435433 & -0.9435436 & -0.9435442 \\ 0 & -0.9435436 & 0.9435438 & -0.9435437 \\ & & & \\ 0 \text { s } & 0.0000004 & 0.0000000 & -0.0000003 \\ 0 & 0.9592638 & -0.9592649 & 0.9592644 \\ 0 & -0.9592639 & 0.9592644 & 0.9592639 \\ 0 & -0.9592643 & -0.9592647 & -0.9592638 \\ 0 & 0.9592641 & 0.9592652 & -0.9592643\end{array}$


15. Optimized Structures at the CAM-QTP-02 Level

The Cartesian coordinates of the structures are listed in Ångström.

\begin{tabular}{|c|c|c|c|}
\hline 0 & 0.0000000 & 0.0000000 & 0.3821174 \\
\hline $\mathrm{H}$ & -0.7645807 & 0.0000000 & -0.1910593 \\
\hline $\mathrm{H}$ & 0.7645807 & 0.0000000 & -0.1910593 \\
\hline $\mathrm{Cr}$ & 0.0000000 & -0.0000000 & 0.0000000 \\
\hline 0 & -0.9287330 & 0.9287330 & 0.9287330 \\
\hline 0 & 0.9287330 & -0.9287330 & 0.9287330 \\
\hline 0 & -0.9287330 & -0.9287330 & -0.9287330 \\
\hline 0 & 0.9287330 & 0.9287330 & -0.9287330 \\
\hline Mo & -0.0000000 & -0.0000000 & 0.0000000 \\
\hline 0 & 1.0071548 & -1.0071548 & 1.0071548 \\
\hline 0 & -1.0071548 & 1.0071548 & 1.0071548 \\
\hline 0 & 1.0071548 & 1.0071548 & -1.0071548 \\
\hline 0 & -1.0071548 & -1.0071548 & -1.0071548 \\
\hline W & -0.0000000 & -0.0000000 & -0.0000000 \\
\hline 0 & -1.0187135 & 1.0187135 & 1.0187135 \\
\hline 0 & 1.0187135 & -1.0187135 & 1.0187135 \\
\hline 0 & 1.0187135 & 1.0187135 & -1.0187135 \\
\hline 0 & -1.0187135 & -1.0187135 & -1.0187135 \\
\hline Mn & 0.0000054 & 0.0000060 & 0.0000019 \\
\hline 0 & 0.8986095 & -0.8986238 & 0.8986139 \\
\hline 0 & -0.8986182 & 0.8986166 & 0.8986157 \\
\hline 0 & -0.8986138 & -0.8986212 & -0.8986106 \\
\hline 0 & 0.8986172 & 0.8986224 & -0.8986209 \\
\hline Tc & 0.0000257 & -0.0000072 & 0.0000110 \\
\hline 0 & 0.9714489 & -0.9714579 & 0.9714597 \\
\hline 0 & -0.9714543 & 0.9714223 & 0.9714129 \\
\hline 0 & -0.9714480 & -0.9714050 & -0.9714258 \\
\hline 0 & 0.9714277 & 0.9714477 & -0.9714578 \\
\hline $\mathrm{Re}$ & 0.0000305 & -0.0000121 & 0.0000249 \\
\hline 0 & 0.9860191 & -0.9860138 & 0.9860024 \\
\hline 0 & -0.9860037 & 0.9859750 & 0.9859268 \\
\hline 0 & -0.9859716 & -0.9859061 & -0.9859699 \\
\hline 0 & 0.9859257 & 0.9859571 & -0.9859842 \\
\hline $\mathrm{Fe}$ & -0.0000001 & -0.0000002 & 0.0000000 \\
\hline 0 & 0.8824924 & 0.8824921 & 0.8824924 \\
\hline 0 & -0.8824920 & -0.8824922 & 0.8824923 \\
\hline 0 & 0.8824920 & -0.8824921 & -0.8824926 \\
\hline 0 & -0.8824923 & 0.8824923 & -0.8824922 \\
\hline
\end{tabular}




$\begin{array}{lrrr}\text { Ru } & -0.0000003 & 0.0000002 & 0.0000003 \\ 0 & 0.9490668 & 0.9490656 & 0.9490649 \\ 0 & -0.9490661 & -0.9490663 & 0.9490651 \\ 0 & 0.9490658 & -0.9490653 & -0.9490651 \\ 0 & -0.9490662 & 0.9490658 & -0.9490653 \\ & & & \\ \text { Os } & -0.0000004 & 0.0000000 & 0.0000002 \\ 0 & 0.9640128 & -0.9640121 & 0.9640124 \\ 0 & -0.9640126 & 0.9640125 & 0.9640128 \\ 0 & -0.9640124 & -0.9640124 & -0.9640129 \\ 0 & 0.9640126 & 0.9640119 & -0.9640125\end{array}$


16. Optimized Structures at the LH07t-SVWN Level

The Cartesian coordinates of the structures are listed in Ångström.

\begin{tabular}{|c|c|c|c|}
\hline 0 & 0.0000000 & 0.0000000 & 0.3846132 \\
\hline $\mathrm{H}$ & -0.7659631 & 0.0000000 & -0.1923071 \\
\hline $\mathrm{H}$ & 0.7659631 & 0.0000000 & -0.1923071 \\
\hline $\mathrm{Cr}$ & -0.0000000 & 0.0000000 & 0.0000000 \\
\hline 0 & -0.9436453 & 0.9436453 & 0.9436453 \\
\hline 0 & 0.9436453 & -0.9436453 & 0.9436453 \\
\hline 0 & -0.9436453 & -0.9436453 & -0.9436453 \\
\hline 0 & 0.9436453 & 0.9436453 & -0.9436453 \\
\hline Mo & -0.0000000 & 0.0000000 & 0.0000000 \\
\hline 0 & 1.0186657 & -1.0186657 & 1.0186657 \\
\hline 0 & -1.0186657 & 1.0186657 & 1.0186657 \\
\hline 0 & 1.0186657 & 1.0186657 & -1.0186657 \\
\hline 0 & -1.0186657 & -1.0186657 & -1.0186657 \\
\hline W & -0.0000000 & 0.0000000 & 0.0000000 \\
\hline 0 & -1.0272748 & 1.0272748 & 1.0272748 \\
\hline 0 & 1.0272748 & -1.0272748 & 1.0272748 \\
\hline 0 & 1.0272748 & 1.0272748 & -1.0272748 \\
\hline 0 & -1.0272748 & -1.0272748 & -1.0272748 \\
\hline Mn & 0.0000044 & 0.0000056 & -0.0000045 \\
\hline 0 & 0.9147195 & -0.9147303 & 0.9147246 \\
\hline 0 & -0.9147281 & 0.9147244 & 0.9147279 \\
\hline 0 & -0.9147284 & -0.9147340 & -0.9147192 \\
\hline 0 & 0.9147326 & 0.9147342 & -0.9147288 \\
\hline Tc & 0.0000236 & -0.0000081 & 0.0000170 \\
\hline 0 & 0.9847002 & -0.9846932 & 0.9846807 \\
\hline 0 & -0.9846818 & 0.9846624 & 0.9846154 \\
\hline 0 & -0.9846503 & -0.9845966 & -0.9846541 \\
\hline 0 & 0.9846082 & 0.9846356 & -0.9846590 \\
\hline $\mathrm{Re}$ & 0.0000238 & -0.0000092 & 0.0000126 \\
\hline 0 & 0.9960487 & -0.9960425 & 0.9960366 \\
\hline 0 & -0.9960311 & 0.9960119 & 0.9959719 \\
\hline 0 & -0.9960049 & -0.9959516 & -0.9960077 \\
\hline 0 & 0.9959635 & 0.9959915 & -0.9960134 \\
\hline $\mathrm{Fe}$ & 0.0000000 & -0.0000000 & -0.0000000 \\
\hline 0 & 0.9001450 & 0.9001447 & 0.9001448 \\
\hline 0 & -0.9001447 & -0.9001449 & 0.9001449 \\
\hline 0 & 0.9001447 & -0.9001448 & -0.9001451 \\
\hline 0 & -0.9001449 & 0.9001450 & -0.9001446 \\
\hline
\end{tabular}




$\begin{array}{lrrr}\text { Ru } & -0.0000000 & 0.0000000 & 0.0000000 \\ 0 & 0.9643472 & 0.9643467 & 0.9643467 \\ 0 & -0.9643469 & -0.9643470 & 0.9643468 \\ 0 & 0.9643468 & -0.9643467 & -0.9643470 \\ 0 & -0.9643471 & 0.9643470 & -0.9643466 \\ & & & \\ 0 \text { s } & -0.0000037 & -0.0000150 & 0.0000121 \\ 0 & 0.9758242 & -0.9758095 & 0.9758117 \\ 0 & -0.9758063 & 0.9758210 & 0.9757997 \\ 0 & -0.9758093 & -0.9758004 & -0.9758218 \\ 0 & 0.9757951 & 0.9758039 & -0.9758018\end{array}$


17. Optimized Structures at the LH12ct-SsirPW92 Level

The Cartesian coordinates of the structures are listed in Ångström.

\begin{tabular}{|c|c|c|c|}
\hline 0 & 0.0000000 & 0.0000000 & 0.3825288 \\
\hline $\mathrm{H}$ & -0.7646408 & 0.0000000 & -0.1912650 \\
\hline $\mathrm{H}$ & 0.7646408 & 0.0000000 & -0.1912650 \\
\hline $\mathrm{Cr}$ & -0.0000000 & -0.0000000 & -0.0000000 \\
\hline 0 & -0.9407724 & 0.9407724 & 0.9407724 \\
\hline 0 & 0.9407724 & -0.9407724 & 0.9407724 \\
\hline 0 & -0.9407724 & -0.9407724 & -0.9407724 \\
\hline 0 & 0.9407724 & 0.9407724 & -0.9407724 \\
\hline Mo & -0.0000000 & 0.0000000 & 0.0000000 \\
\hline 0 & 1.0160964 & -1.0160964 & 1.0160964 \\
\hline 0 & -1.0160964 & 1.0160964 & 1.0160964 \\
\hline 0 & 1.0160964 & 1.0160964 & -1.0160964 \\
\hline 0 & -1.0160964 & -1.0160964 & -1.0160964 \\
\hline W & -0.0000000 & 0.0000000 & 0.0000000 \\
\hline 0 & -1.0253598 & 1.0253598 & 1.0253598 \\
\hline 0 & 1.0253598 & -1.0253598 & 1.0253598 \\
\hline 0 & 1.0253598 & 1.0253598 & -1.0253598 \\
\hline 0 & -1.0253598 & -1.0253598 & -1.0253598 \\
\hline Mn & 0.0000036 & 0.0000077 & -0.0000034 \\
\hline 0 & 0.9112868 & -0.9112988 & 0.9112916 \\
\hline 0 & -0.9112967 & 0.9112926 & 0.9112973 \\
\hline 0 & -0.9112946 & -0.9113024 & -0.9112875 \\
\hline 0 & 0.9113009 & 0.9113010 & -0.9112980 \\
\hline Tc & 0.0000245 & -0.0000071 & 0.0000148 \\
\hline 0 & 0.9813912 & -0.9813950 & 0.9813759 \\
\hline 0 & -0.9813747 & 0.9813618 & 0.9813104 \\
\hline 0 & -0.9813441 & -0.9812989 & -0.9813465 \\
\hline 0 & 0.9813030 & 0.9813391 & -0.9813546 \\
\hline $\mathrm{Re}$ & 0.0000118 & -0.0000108 & 0.0000163 \\
\hline 0 & 0.9934746 & -0.9934566 & 0.9934498 \\
\hline 0 & -0.9934494 & 0.9934399 & 0.9933967 \\
\hline 0 & -0.9934208 & -0.9933749 & -0.9934359 \\
\hline 0 & 0.9933839 & 0.9934023 & -0.9934269 \\
\hline $\mathrm{Fe}$ & -0.0000001 & 0.0000000 & 0.0000002 \\
\hline 0 & 0.8962526 & 0.8962518 & 0.8962509 \\
\hline 0 & -0.8962527 & -0.8962523 & 0.8962517 \\
\hline 0 & 0.8962523 & -0.8962515 & -0.8962517 \\
\hline 0 & -0.8962521 & 0.8962520 & -0.8962512 \\
\hline
\end{tabular}




$\begin{array}{lrrr}\mathrm{Ru} & 0.0000000 & -0.0000000 & 0.0000000 \\ 0 & 0.9603275 & 0.9603271 & 0.9603272 \\ 0 & -0.9603272 & -0.9603273 & 0.9603273 \\ 0 & 0.9603271 & -0.9603271 & -0.9603275 \\ 0 & -0.9603274 & 0.9603274 & -0.9603270 \\ & & & \\ & & & \\ \text { Os } & 0.0000008 & 0.0000006 & -0.0000007 \\ 0 & 0.9725641 & -0.9725650 & 0.9725653 \\ 0 & -0.9725651 & 0.9725639 & 0.9725651 \\ 0 & -0.9725652 & -0.9725649 & -0.9725641 \\ 0 & 0.9725654 & 0.9725654 & -0.9725655\end{array}$


18. Optimized Structures at the LH14t-calPBE Level

The Cartesian coordinates of the structures are listed in Ångström.

\begin{tabular}{|c|c|c|c|}
\hline 0 & 0.0000000 & 0.0000000 & 0.3847428 \\
\hline $\mathrm{H}$ & -0.7653412 & 0.0000000 & -0.1923720 \\
\hline $\mathrm{H}$ & 0.7653412 & 0.0000000 & -0.1923720 \\
\hline $\mathrm{Cr}$ & 0.0000000 & 0.0000000 & 0.0000000 \\
\hline 0 & -0.9429593 & 0.9429593 & 0.9429593 \\
\hline 0 & 0.9429593 & -0.9429593 & 0.9429593 \\
\hline 0 & -0.9429593 & -0.9429593 & -0.9429593 \\
\hline 0 & 0.9429593 & 0.9429593 & -0.9429593 \\
\hline Mo & -0.0000000 & 0.0000000 & 0.0000000 \\
\hline 0 & 1.0181560 & -1.0181560 & 1.0181560 \\
\hline 0 & -1.0181560 & 1.0181560 & 1.0181560 \\
\hline 0 & 1.0181560 & 1.0181560 & -1.0181560 \\
\hline 0 & -1.0181560 & -1.0181560 & -1.0181560 \\
\hline W & 0.0000000 & 0.0000000 & 0.0000000 \\
\hline 0 & -1.0270219 & 1.0270219 & 1.0270219 \\
\hline 0 & 1.0270219 & -1.0270219 & 1.0270219 \\
\hline 0 & 1.0270219 & 1.0270219 & -1.0270219 \\
\hline 0 & -1.0270219 & -1.0270219 & -1.0270219 \\
\hline Mn & 0.0000040 & 0.0000057 & -0.0000044 \\
\hline 0 & 0.9142072 & -0.9142172 & 0.9142118 \\
\hline 0 & -0.9142157 & 0.9142118 & 0.9142158 \\
\hline 0 & -0.9142157 & -0.9142212 & -0.9142070 \\
\hline 0 & 0.9142203 & 0.9142209 & -0.9142161 \\
\hline Tc & 0.0000239 & -0.0000083 & 0.0000174 \\
\hline 0 & 0.9843906 & -0.9843833 & 0.9843710 \\
\hline 0 & -0.9843722 & 0.9843523 & 0.9843052 \\
\hline 0 & -0.9843405 & -0.9842861 & -0.9843443 \\
\hline 0 & 0.9842982 & 0.9843254 & -0.9843493 \\
\hline $\mathrm{Re}$ & 0.0000212 & -0.0000147 & 0.0000166 \\
\hline 0 & 0.9959414 & -0.9959281 & 0.9959240 \\
\hline 0 & -0.9959164 & 0.9959032 & 0.9958562 \\
\hline 0 & -0.9958921 & -0.9958360 & -0.9959001 \\
\hline 0 & 0.9958459 & 0.9958756 & -0.9958967 \\
\hline $\mathrm{Fe}$ & 0.0000000 & 0.0000000 & -0.0000000 \\
\hline 0 & 0.8997295 & 0.8997292 & 0.8997294 \\
\hline 0 & -0.8997292 & -0.8997294 & 0.8997294 \\
\hline 0 & 0.8997291 & -0.8997293 & -0.8997296 \\
\hline 0 & -0.8997295 & 0.8997295 & -0.8997292 \\
\hline
\end{tabular}




$\begin{array}{lrrr}\mathrm{Ru} & 0.0000000 & -0.0000000 & -0.0000000 \\ \mathrm{O} & 0.9641632 & 0.9641628 & 0.9641630 \\ 0 & -0.9641628 & -0.9641630 & 0.9641631 \\ 0 & 0.9641628 & -0.9641630 & -0.9641633 \\ 0 & -0.9641631 & 0.9641632 & -0.9641628 \\ & & & \\ & & & \\ \text { Os } & 0.0000239 & 0.0000125 & 0.0000045 \\ 0 & 0.9757677 & -0.9757946 & 0.9757824 \\ 0 & -0.9757928 & 0.9757630 & 0.9757702 \\ 0 & -0.9757748 & -0.9757643 & -0.9757592 \\ 0 & 0.9757760 & 0.9757835 & -0.9757978\end{array}$


19. Optimized Structures at the LH2Ot Level

The Cartesian coordinates of the structures are listed in Ångström.

\begin{tabular}{|c|c|c|c|}
\hline 0 & 0.0000000 & 0.0000000 & 0.3811559 \\
\hline $\mathrm{H}$ & -0.7643959 & 0.0000000 & -0.1905785 \\
\hline $\mathrm{H}$ & 0.7643959 & 0.0000000 & -0.1905785 \\
\hline $\mathrm{Cr}$ & 0.0000000 & -0.0000000 & 0.0000000 \\
\hline 0 & -0.9381825 & 0.9381825 & 0.9381825 \\
\hline 0 & 0.9381825 & -0.9381825 & 0.9381825 \\
\hline 0 & -0.9381825 & -0.9381825 & -0.9381825 \\
\hline 0 & 0.9381825 & 0.9381825 & -0.9381825 \\
\hline Mo & -0.0000000 & 0.0000000 & 0.0000000 \\
\hline 0 & 1.0140707 & -1.0140707 & 1.0140707 \\
\hline 0 & -1.0140707 & 1.0140707 & 1.0140707 \\
\hline 0 & 1.0140707 & 1.0140707 & -1.0140707 \\
\hline 0 & -1.0140707 & -1.0140707 & -1.0140707 \\
\hline W & -0.0000000 & 0.0000000 & 0.0000000 \\
\hline 0 & -1.0235283 & 1.0235283 & 1.0235283 \\
\hline 0 & 1.0235283 & -1.0235283 & 1.0235283 \\
\hline 0 & 1.0235283 & 1.0235283 & -1.0235283 \\
\hline 0 & -1.0235283 & -1.0235283 & -1.0235283 \\
\hline Mn & 0.0000036 & 0.0000080 & -0.0000037 \\
\hline 0 & 0.9089139 & -0.9089263 & 0.9089189 \\
\hline 0 & -0.9089241 & 0.9089198 & 0.9089249 \\
\hline 0 & -0.9089220 & -0.9089301 & -0.9089146 \\
\hline 0 & 0.9089286 & 0.9089286 & -0.9089256 \\
\hline Tc & 0.0000245 & -0.0000085 & 0.0000168 \\
\hline 0 & 0.9796532 & -0.9796460 & 0.9796349 \\
\hline 0 & -0.9796355 & 0.9796140 & 0.9795685 \\
\hline 0 & -0.9796051 & -0.9795494 & -0.9796069 \\
\hline 0 & 0.9795629 & 0.9795899 & -0.9796134 \\
\hline $\mathrm{Re}$ & 0.0000168 & -0.0000140 & 0.0000122 \\
\hline 0 & 0.9918441 & -0.9918282 & 0.9918276 \\
\hline 0 & -0.9918184 & 0.9918074 & 0.9917657 \\
\hline 0 & -0.9917989 & -0.9917467 & -0.9918059 \\
\hline 0 & 0.9917564 & 0.9917815 & -0.9917996 \\
\hline $\mathrm{Fe}$ & 0.0000000 & 0.0000000 & -0.0000000 \\
\hline 0 & 0.8939508 & 0.8939505 & 0.8939506 \\
\hline 0 & -0.8939505 & -0.8939507 & 0.8939507 \\
\hline 0 & 0.8939505 & -0.8939506 & -0.8939509 \\
\hline 0 & -0.8939508 & 0.8939508 & -0.8939504 \\
\hline
\end{tabular}




$\begin{array}{lrrr}\text { Ru } & 0.0000000 & -0.0000000 & -0.0000000 \\ 0 & 0.9587042 & 0.9587038 & 0.9587040 \\ 0 & -0.9587038 & -0.9587040 & 0.9587040 \\ 0 & 0.9587038 & -0.9587039 & -0.9587042 \\ 0 & -0.9587041 & 0.9587041 & -0.9587038 \\ & & & \\ \text { Os } & -0.0000001 & 0.0000000 & 0.0000000 \\ 0 & 0.9710362 & -0.9710360 & 0.9710362 \\ 0 & -0.9710362 & 0.9710361 & 0.9710362 \\ 0 & -0.9710362 & -0.9710361 & -0.9710363 \\ 0 & 0.9710362 & 0.9710360 & -0.9710361\end{array}$


20. Optimized Structures at the mPSTS Level

The Cartesian coordinates of the structures are listed in Ångström.

\begin{tabular}{|c|c|c|c|}
\hline 0 & 0.0000000 & 0.0000000 & 0.3921286 \\
\hline $\mathrm{H}$ & -0.7620838 & 0.0000000 & -0.1960648 \\
\hline $\mathrm{H}$ & 0.7620838 & 0.0000000 & -0.1960648 \\
\hline $\mathrm{Cr}$ & 0.0000000 & -0.0000000 & 0.0000000 \\
\hline 0 & -0.9489414 & 0.9489414 & 0.9489414 \\
\hline 0 & 0.9489414 & -0.9489414 & 0.9489414 \\
\hline 0 & -0.9489414 & -0.9489414 & -0.9489414 \\
\hline 0 & 0.9489414 & 0.9489414 & -0.9489414 \\
\hline Mo & 0.0000000 & 0.0000000 & -0.0000000 \\
\hline 0 & 1.0241851 & -1.0241851 & 1.0241851 \\
\hline 0 & -1.0241851 & 1.0241851 & 1.0241851 \\
\hline 0 & 1.0241851 & 1.0241851 & -1.0241851 \\
\hline 0 & -1.0241851 & -1.0241851 & -1.0241851 \\
\hline W & -0.0000000 & 0.0000000 & 0.0000000 \\
\hline 0 & -1.0324845 & 1.0324845 & 1.0324845 \\
\hline 0 & 1.0324845 & -1.0324845 & 1.0324845 \\
\hline 0 & 1.0324845 & 1.0324845 & -1.0324845 \\
\hline 0 & -1.0324845 & -1.0324845 & -1.0324845 \\
\hline Mn & 0.0000032 & 0.0000063 & -0.0000055 \\
\hline 0 & 0.9206264 & -0.9206366 & 0.9206308 \\
\hline 0 & -0.9206346 & 0.9206310 & 0.9206350 \\
\hline 0 & -0.9206346 & -0.9206407 & -0.9206256 \\
\hline 0 & 0.9206396 & 0.9206400 & -0.9206347 \\
\hline Tc & 0.0000133 & -0.0000100 & 0.0000063 \\
\hline 0 & 0.9911571 & -0.9911388 & 0.9911351 \\
\hline 0 & -0.9911272 & 0.9911224 & 0.9910770 \\
\hline 0 & -0.9911034 & -0.9910571 & -0.9911166 \\
\hline 0 & 0.9910602 & 0.9910835 & -0.9911018 \\
\hline $\operatorname{Re}$ & 0.0000174 & -0.0000081 & 0.0000098 \\
\hline 0 & 1.0017084 & -1.0017037 & 1.0016947 \\
\hline 0 & -1.0016882 & 1.0016805 & 1.0016386 \\
\hline 0 & -1.0016623 & -1.0016217 & -1.0016724 \\
\hline 0 & 1.0016247 & 1.0016530 & -1.0016707 \\
\hline $\mathrm{Fe}$ & -0.0000000 & -0.0000000 & 0.0000000 \\
\hline 0 & 0.9064706 & 0.9064703 & 0.9064703 \\
\hline 0 & -0.9064704 & -0.9064705 & 0.9064705 \\
\hline 0 & 0.9064703 & -0.9064703 & -0.9064707 \\
\hline 0 & -0.9064705 & 0.9064706 & -0.9064702 \\
\hline
\end{tabular}




$\begin{array}{lrrr}\mathrm{Ru} & 0.0000000 & -0.0000000 & 0.0000000 \\ 0 & 0.9718806 & 0.9718803 & 0.9718804 \\ 0 & -0.9718803 & -0.9718805 & 0.9718805 \\ 0 & 0.9718802 & -0.9718803 & -0.9718807 \\ 0 & -0.9718805 & 0.9718805 & -0.9718802 \\ & & & \\ 0 \mathrm{~s} & 0.0000001 & -0.0000000 & -0.0000000 \\ 0 & 0.9822386 & -0.9822389 & 0.9822388 \\ 0 & -0.9822387 & 0.9822387 & 0.9822386 \\ 0 & -0.9822387 & -0.9822388 & -0.9822386 \\ 0 & 0.9822387 & 0.9822389 & -0.9822388\end{array}$


21. Optimized Structures at the LHJ14 Level

The Cartesian coordinates of the structures are listed in Ångström.

\begin{tabular}{|c|c|c|c|}
\hline 0 & 0.0000000 & 0.0000000 & 0.3878487 \\
\hline $\mathrm{H}$ & -0.7641174 & 0.0000000 & -0.1939249 \\
\hline $\mathrm{H}$ & 0.7641174 & -0.0000000 & -0.1939249 \\
\hline $\mathrm{Cr}$ & -0.0000000 & 0.0000000 & 0.0000000 \\
\hline 0 & -0.9460299 & 0.9460299 & 0.9460299 \\
\hline 0 & 0.9460299 & -0.9460299 & 0.9460299 \\
\hline 0 & -0.9460299 & -0.9460299 & -0.9460299 \\
\hline 0 & 0.9460299 & 0.9460299 & -0.9460299 \\
\hline Mo & -0.0000000 & 0.0000000 & 0.0000000 \\
\hline 0 & 1.0198083 & -1.0198083 & 1.0198083 \\
\hline 0 & -1.0198083 & 1.0198083 & 1.0198083 \\
\hline 0 & 1.0198083 & 1.0198083 & -1.0198083 \\
\hline 0 & -1.0198083 & -1.0198083 & -1.0198083 \\
\hline W & -0.0000000 & 0.0000000 & 0.0000000 \\
\hline 0 & -1.0287821 & 1.0287821 & 1.0287821 \\
\hline 0 & 1.0287821 & -1.0287821 & 1.0287821 \\
\hline 0 & 1.0287821 & 1.0287821 & -1.0287821 \\
\hline 0 & -1.0287821 & -1.0287821 & -1.0287821 \\
\hline Mn & 0.0000026 & 0.0000064 & -0.0000065 \\
\hline 0 & 0.9186805 & -0.9186915 & 0.9186855 \\
\hline 0 & -0.9186885 & 0.9186867 & 0.9186906 \\
\hline 0 & -0.9186891 & -0.9186972 & -0.9186806 \\
\hline 0 & 0.9186945 & 0.9186956 & -0.9186889 \\
\hline Tc & 0.0000222 & -0.0000072 & 0.0000169 \\
\hline 0 & 0.9875207 & -0.9875151 & 0.9875002 \\
\hline 0 & -0.9874996 & 0.9874827 & 0.9874319 \\
\hline 0 & -0.9874669 & -0.9874142 & -0.9874725 \\
\hline 0 & 0.9874235 & 0.9874537 & -0.9874765 \\
\hline $\mathrm{Re}$ & 0.0000180 & -0.0000130 & 0.0000157 \\
\hline 0 & 0.9987364 & -0.9987247 & 0.9987173 \\
\hline 0 & -0.9987120 & 0.9987028 & 0.9986555 \\
\hline 0 & -0.9986861 & -0.9986370 & -0.9986968 \\
\hline 0 & 0.9986437 & 0.9986719 & -0.9986917 \\
\hline $\mathrm{Fe}$ & -0.0000000 & 0.0000000 & 0.0000000 \\
\hline 0 & 0.9052590 & 0.9052587 & 0.9052587 \\
\hline 0 & -0.9052588 & -0.9052589 & 0.9052588 \\
\hline 0 & 0.9052587 & -0.9052588 & -0.9052590 \\
\hline 0 & -0.9052590 & 0.9052590 & -0.9052586 \\
\hline
\end{tabular}




$\begin{array}{lrrr}\mathrm{Ru} & 0.0000000 & -0.0000000 & -0.0000000 \\ \mathrm{O} & 0.9687196 & 0.9687193 & 0.9687195 \\ 0 & -0.9687193 & -0.9687195 & 0.9687195 \\ 0 & 0.9687193 & -0.9687194 & -0.9687198 \\ 0 & -0.9687196 & 0.9687196 & -0.9687192 \\ & & & \\ & & & \\ \text { Os } & 0.0000000 & 0.0000000 & -0.0000000 \\ 0 & 0.9796702 & -0.9796703 & 0.9796703 \\ 0 & -0.9796703 & 0.9796702 & 0.9796703 \\ 0 & -0.9796702 & -0.9796703 & -0.9796703 \\ 0 & 0.9796703 & 0.9796703 & -0.9796703\end{array}$




\section{NMR Shieldings and Shifts of Xenon Fluorides}

Xenon fluorides were studied with the scalar-relativistic DLU-X2C Hamiltonian [94-96, 100]. The same settings as in Sec. $\mathrm{S} 5 \mathrm{C}$ are applied. However, the root mean square of the density was required to be smaller than $10^{-8}$ a.u. in the SCF iterations. Structures were optimized with symmetry constraints in line with Refs. 100 and $105: \mathrm{XeF}_{2}\left(D_{\infty h}\right), \mathrm{XeF}_{4}\left(D_{4 h}\right)$ and $\mathrm{XeF}_{6}\left(O_{h}\right)$. For the latter, there is substantial evidence that the global minimum is the $C_{3 v}$ structure [106]. However, the energy difference is small and structure conversion is likely to occur as indicated by the experimental NMR spectra, which shows only one $\mathrm{F}$ peak [107]. Geometries were considered converged when the energy change between optimization steps falls below $10^{-6} E_{h}$. All DFT structures were confirmed to be (local) minima by numerical frequency calculations, however, the structures optimized with BH\&HLYP, CAM-QTP-00, and CAM-QTP-02 are saddle points like HF.

The experimental shifts are measured compared to neat $\mathrm{XeOF}_{4}$ in the gas-phase while we use the xenon atom to compute the shifts in this work. We use the shift of xenon gas at infinite dilution $(-5460 \mathrm{ppm})$ with respect to neat $\mathrm{XeOF}_{4}$ [108] to convert the experimental shifts.

\section{NMR Data}

TABLE S62. Comparison of ${ }^{129}$ Xe NMR shielding constants (in ppm) at various levels. LH07t, LH12ct, and LH14t refer to the functionals LH07t-SVWN, LH12ct-SsirPW92, and LH14t-calPBE, respectively.

\begin{tabular}{|c|c|c|c|c|c|c|c|c|c|c|c|c|c|c|c|c|c|c|}
\hline & $\mathrm{HF}$ & KT3 & BP86 & PBE & TPSS & BH\&HLYP & B3LYP & PBE0 & TPSSh & LC- $\omega$ PBE & $\omega \mathrm{B} 97 \mathrm{X}-\mathrm{D}$ & CAM-B3LYP & LH07t & LH12ct & LH14t & LH20t & mPSTS & LHJ14 \\
\hline $\mathrm{Xe}$ & 5847 & 5854 & 5848 & 5848 & 5892 & 5848 & 5848 & 5848 & 5888 & 5847 & 5848 & 5848 & 5972 & 5985 & 5879 & 5835 & 5884 & 5750 \\
\hline $\mathrm{XeF}_{2}$ & 2033 & 2332 & 2177 & 2204 & 2339 & 1963 & 1984 & 2153 & 2300 & 1959 & 1966 & 1879 & 2118 & 2136 & 2116 & 2085 & 2319 & 2020 \\
\hline $\mathrm{XeF}_{4}$ & 534 & -144 & -530 & -480 & -231 & 18 & -383 & -76 & -93 & -140 & -224 & -316 & -198 & -71 & -147 & -27 & -41 & -384 \\
\hline $\mathrm{XeF}_{6}$ & 1177 & -46 & -477 & -416 & -238 & 405 & -235 & 115 & -45 & 321 & 115 & 56 & -16 & 147 & 36 & 236 & -14 & -201 \\
\hline
\end{tabular}

TABLE S63. Comparison of ${ }^{129}$ Xe NMR shifts (in ppm) at various levels. CCSD(T) results are taken from Ref. 105 The uncontracted ANO-RCC basis set [109] is used therein and all electrons are correlated. The difference between the CCSD(T) results and the experimental findings can mainly be addressed to spin-orbit coupling.

\begin{tabular}{|c|c|c|c|c|c|c|c|c|c|c|c|c|c|c|c|c|c|c|c|}
\hline $\mathrm{HF}$ & KT3 & BP86 & PBE & TPSS & BH\&HLYP & B3LYP & PBE0 & TPSSh & LC- $\omega$ PBE & $\omega \mathrm{B} 97 \mathrm{X}-\mathrm{D}$ & CAM-B3LYP & LH07t & LH12ct & LH14t & LH20t & mPSTS & LHJ14 & $\operatorname{CCSD}(\mathrm{T})$ & Exp. \\
\hline & & 3671 & 3644 & 553 & 3885 & 864 & 3695 & 3588 & 3888 & 3 & 3969 & 3854 & 3840 & 3763 & 3751 & 3565 & 3730 & 564 & 3386 \\
\hline 45312 & 5998 & 6378 & 6328 & 6122 & 6231 & 5830 & 5924 & 5981 & 5987 & 6072 & 6171 & 6164 & 6057 & 6026 & 5862 & 5924 & 6135 & 5509 & 5623 \\
\hline $\mathrm{XeF}_{6} 4669$ & 5900 & 6326 & 6264 & 6129 & 6083 & 5443 & 5733 & 5933 & 5527 & 5733 & 5793 & 5989 & 5838 & 5843 & 5600 & 5897 & 5952 & 5258 & 5425 \\
\hline
\end{tabular}

TABLE S64. Comparison of ${ }^{129} \mathrm{Xe}$ NMR shielding constants (in ppm) at various levels.

\begin{tabular}{lccrrrrrrrr}
\hline & CAM-QTP-00 & CAM-QTP-02 & cTPSS & cTPSSh & cLH07t & cLH12ct & cLH14t & cLH20t & cmPSTS & cLHJ14 \\
\hline $\mathrm{Xe}$ & 5848 & 5848 & 5847 & 5847 & 5846 & 5846 & 5847 & 5847 & 5847 & 5848 \\
$\mathrm{XeF}_{2}$ & 1937 & 1858 & 2331 & 2291 & 2086 & 2097 & 2104 & 2076 & 2312 & 2028 \\
$\mathrm{XeF}_{4}$ & 114 & -133 & -225 & -89 & -195 & -72 & -149 & -36 & -35 & -406 \\
$\mathrm{XeF}_{6}$ & 659 & 414 & -243 & -49 & -10 & 153 & 39 & 237 & -17 & -206 \\
\hline
\end{tabular}

TABLE S65. Comparison of ${ }^{129}$ Xe NMR shifts (in ppm) at various levels. CCSD(T) results are taken from Ref. 105

\begin{tabular}{|c|c|c|c|c|c|c|c|c|c|c|c|c|}
\hline & CAM-QTP-00 & CAM-QTP-02 & cTPSS & cTPSSh & cLH07t & cLH12ct & cLH14t & cLH20t & cmPSTS & cLHJ14 & $\operatorname{CCSD}(\mathrm{T})$ & Exp. \\
\hline $\mathrm{XeF}_{2}$ & 3911 & 3990 & 3516 & 3556 & 3761 & 3749 & 3743 & 3771 & 3535 & 3820 & 3564 & 3386 \\
\hline $\mathrm{XeF}_{4}$ & 5733 & 5981 & 6071 & 5935 & 6041 & 5918 & 5995 & 5883 & 5882 & 6255 & 5509 & 5623 \\
\hline $\mathrm{XeF}_{6}$ & 5189 & 5434 & 6089 & 5896 & 5857 & 5693 & 5808 & 5610 & 5863 & 6055 & 5258 & 5425 \\
\hline
\end{tabular}


2. Optimized Structures at the HF Level

The Cartesian coordinates of the structures are listed in Ångström.

$\begin{array}{lrrr}\text { Xe } & -0.0000000 & 0.0000000 & 0.0000000 \\ \text { F } & 0.0000000 & 0.0000000 & 1.9331114 \\ \text { F } & 0.0000000 & -0.0000000 & -1.9331114 \\ & & & \\ & & & \\ \text { Xe } & 0.0000000 & 0.0000000 & -0.0000000 \\ \text { F } & 1.3371109 & -1.3371109 & 0.0000000 \\ \text { F } & -1.3371109 & 1.3371109 & 0.0000000 \\ \text { F } & 1.3371109 & 1.3371109 & 0.0000000 \\ \text { F } & -1.3371109 & -1.3371109 & 0.0000000 \\ & & & \\ & & & \\ \text { Xe } & -0.0000000 & -0.0000000 & -0.0000000 \\ \text { F } & -0.0000000 & 1.8821638 & 0.0000000 \\ \text { F } & 0.0000000 & -1.8821638 & -0.0000000 \\ F & 0.0000000 & 0.0000000 & 1.8821638 \\ F & 0.0000000 & 0.0000000 & -1.8821638 \\ F & 1.8821638 & 0.0000000 & -0.0000000 \\ F & -1.8821638 & -0.0000000 & 0.0000000\end{array}$

\section{Optimized Structures at the KT3 Level}

The Cartesian coordinates of the structures are listed in Ångström.

$\begin{array}{lrrr}\text { Xe } & -0.0000000 & 0.0000000 & 0.0000000 \\ \text { F } & 0.0000000 & 0.0000000 & 1.9975774 \\ \text { F } & 0.0000000 & 0.0000000 & -1.9975774 \\ & & & \\ & & & \\ \text { Xe } & -0.0000000 & 0.0000000 & -0.0000000 \\ \text { F } & 1.3853173 & -1.3853173 & -0.0000000 \\ F & -1.3853173 & 1.3853173 & -0.0000000 \\ F & 1.3853173 & 1.3853173 & 0.0000000 \\ F & -1.3853173 & -1.3853173 & -0.0000000 \\ & & & \\ & & & \\ \text { Xe } & -0.0000000 & -0.0000000 & -0.0000000 \\ F & -0.0000000 & 1.9426527 & 0.0000000 \\ F & 0.0000000 & -1.9426527 & 0.0000000 \\ F & 0.0000000 & 0.0000000 & 1.9426527 \\ F & 0.0000000 & 0.0000000 & -1.9426527 \\ F & 1.9426527 & 0.0000000 & -0.0000000 \\ F & -1.9426527 & -0.0000000 & 0.0000000\end{array}$


4. Optimized Structures at the BP86 Level

The Cartesian coordinates of the structures are listed in Ångström.

$\begin{array}{lrrr}\text { Xe } & -0.0000000 & 0.0000000 & 0.0000000 \\ \text { F } & 0.0000000 & -0.0000000 & 2.0280944 \\ \text { F } & 0.0000000 & 0.0000000 & -2.0280944 \\ & & & \\ & & & \\ \text { Xe } & -0.0000000 & 0.0000000 & 0.0000000 \\ \text { F } & 1.4062532 & -1.4062532 & -0.0000000 \\ \text { F } & -1.4062532 & 1.4062532 & 0.0000000 \\ \text { F } & 1.4062532 & 1.4062532 & 0.0000000 \\ \text { F } & -1.4062532 & -1.4062532 & 0.0000000 \\ & & & \\ & & & \\ \text { Xe } & -0.0000000 & -0.0000000 & 0.0000000 \\ \text { F } & -0.0000000 & 1.9695529 & -0.0000000 \\ \text { F } & 0.0000000 & -1.9695529 & -0.0000000 \\ \text { F } & 0.0000000 & -0.0000000 & 1.9695529 \\ F & 0.0000000 & -0.0000000 & -1.9695529 \\ \text { F } & 1.9695529 & 0.0000000 & -0.0000000 \\ \text { F } & -1.9695529 & -0.0000000 & -0.0000000\end{array}$

\section{Optimized Structures at the PBE Level}

The Cartesian coordinates of the structures are listed in Ångström.

$\begin{array}{lrrr}\text { Xe } & -0.0000000 & 0.0000000 & -0.0000000 \\ \text { F } & 0.0000000 & 0.0000000 & 2.0261407 \\ \text { F } & 0.0000000 & 0.0000000 & -2.0261407 \\ & & & \\ \text { Xe } & -0.0000000 & 0.0000000 & -0.0000000 \\ \text { F } & 1.4050414 & -1.4050414 & 0.0000000 \\ \text { F } & -1.4050414 & 1.4050414 & 0.0000000 \\ F & 1.4050414 & 1.4050414 & 0.0000000 \\ F & -1.4050414 & -1.4050414 & 0.0000000 \\ & & & \\ & & & \\ \text { Xe } & 0.0000000 & 0.0000000 & -0.0000000 \\ F & -0.0000000 & 1.9682952 & 0.0000000 \\ F & 0.0000000 & -1.9682952 & -0.0000000 \\ F & 0.0000000 & -0.0000000 & 1.9682952 \\ F & 0.0000000 & -0.0000000 & -1.9682952 \\ F & 1.9682952 & 0.0000000 & 0.0000000 \\ F & -1.9682952 & -0.0000000 & 0.0000000\end{array}$


6. Optimized Structures at the TPSS Level

The Cartesian coordinates of the structures are listed in Ångström.

$\begin{array}{lrrr}\text { Xe } & -0.0000000 & -0.0000000 & 0.0000000 \\ \mathrm{~F} & 0.0000000 & 0.0000000 & 2.0190949 \\ \mathrm{~F} & 0.0000000 & 0.0000000 & -2.0190949 \\ & & & \\ \mathrm{Xe} & -0.0000000 & 0.0000000 & 0.0000000 \\ \mathrm{~F} & 1.4003025 & -1.4003025 & 0.0000000 \\ \mathrm{~F} & -1.4003025 & 1.4003025 & 0.0000000 \\ \mathrm{~F} & 1.4003025 & 1.4003025 & 0.0000000 \\ \mathrm{~F} & -1.4003025 & -1.4003025 & 0.0000000 \\ & & & \\ & & & \\ \mathrm{Xe} & 0.0000000 & 0.0000000 & 0.0000000 \\ \mathrm{~F} & -0.0000000 & 1.9627809 & 0.0000000 \\ \mathrm{~F} & 0.0000000 & -1.9627809 & -0.0000000 \\ \mathrm{~F} & -0.0000000 & 0.0000000 & 1.9627809 \\ \mathrm{~F} & 0.0000000 & 0.0000000 & -1.9627809 \\ \mathrm{~F} & 1.9627809 & 0.0000000 & 0.0000000 \\ \mathrm{~F} & -1.9627809 & -0.0000000 & 0.0000000 \\ & & & \\ & & & 7 .\end{array}$

The Cartesian coordinates of the structures are listed in Ångström.

$\begin{array}{lrrr}\text { Xe } & -0.0000000 & 0.0000000 & 0.0000000 \\ \text { F } & 0.0000000 & 0.0000000 & 1.9631181 \\ \text { F } & 0.0000000 & 0.0000000 & -1.9631181 \\ & & & \\ & & & \\ \text { Xe } & -0.0000000 & 0.0000000 & 0.0000000 \\ \text { F } & 1.3590883 & -1.3590883 & 0.0000000 \\ \text { F } & -1.3590883 & 1.3590883 & 0.0000000 \\ F & 1.3590883 & 1.3590883 & 0.0000000 \\ F & -1.3590883 & -1.3590883 & -0.0000000 \\ & & & \\ & & & \\ \text { Xe } & 0.0000000 & 0.0000000 & -0.0000000 \\ F & -0.0000000 & 1.9076234 & 0.0000000 \\ F & 0.0000000 & -1.9076234 & -0.0000000 \\ F & 0.0000000 & -0.0000000 & 1.9076234 \\ F & 0.0000000 & -0.0000000 & -1.9076234 \\ F & 1.9076234 & 0.0000000 & -0.0000000 \\ F & -1.9076234 & -0.0000000 & 0.0000000\end{array}$


8. Optimized Structures at the B3LYP Level

The Cartesian coordinates of the structures are listed in Ångström.

$\begin{array}{lrrr}\text { Xe } & -0.0000000 & 0.0000000 & -0.0000000 \\ \text { F } & 0.0000000 & -0.0000000 & 2.0071353 \\ \text { F } & 0.0000000 & -0.0000000 & -2.0071353 \\ & & & \\ & & & \\ \text { Xe } & -0.0000000 & 0.0000000 & -0.0000000 \\ \text { F } & 1.3899949 & -1.3899949 & 0.0000000 \\ \text { F } & -1.3899949 & 1.3899949 & 0.0000000 \\ \text { F } & 1.3899949 & 1.3899949 & 0.0000000 \\ \text { F } & -1.3899949 & -1.3899949 & -0.0000000 \\ & & & \\ & & & \\ \text { Xe } & -0.0000000 & -0.0000000 & 0.0000000 \\ \text { F } & -0.0000000 & 1.9471473 & -0.0000000 \\ \text { F } & 0.0000000 & -1.9471473 & 0.0000000 \\ \text { F } & 0.0000000 & -0.0000000 & 1.9471473 \\ \text { F } & 0.0000000 & -0.0000000 & -1.9471473 \\ \text { F } & 1.9471473 & 0.0000000 & 0.0000000 \\ \text { F } & -1.9471473 & -0.0000000 & -0.0000000\end{array}$

9. Optimized Structures at the PBEO Level

The Cartesian coordinates of the structures are listed in Ångström.

$\begin{array}{lrrr}\text { Xe } & -0.0000000 & -0.0000000 & -0.0000000 \\ \text { F } & 0.0000000 & -0.0000000 & 1.9814957 \\ \text { F } & 0.0000000 & 0.0000000 & -1.9814957 \\ & & & \\ & & & \\ \text { Xe } & -0.0000000 & 0.0000000 & -0.0000000 \\ \text { F } & 1.3731739 & -1.3731739 & 0.0000000 \\ \text { F } & -1.3731739 & 1.3731739 & 0.0000000 \\ \text { F } & 1.3731739 & 1.3731739 & 0.0000000 \\ \text { F } & -1.3731739 & -1.3731739 & 0.0000000 \\ & & & \\ & & & \\ \text { Xe } & -0.0000000 & -0.0000000 & -0.0000000 \\ F & -0.0000000 & 1.9262724 & 0.0000000 \\ F & 0.0000000 & -1.9262724 & -0.0000000 \\ F & 0.0000000 & -0.0000000 & 1.9262724 \\ F & 0.0000000 & -0.0000000 & -1.9262724 \\ F & 1.9262724 & 0.0000000 & -0.0000000 \\ F & -1.9262724 & -0.0000000 & 0.0000000\end{array}$




\section{Optimized Structures at the TPSSh Level}

The Cartesian coordinates of the structures are listed in Ångström.
Xe $\quad-0.0000000$
0.0000000
0.0000000
$\begin{array}{ll}F & 0.0000000 \\ F & 0.0000000\end{array}$
0.0000000
2.0013055
0.0000000
$-2.0013055$
$\begin{array}{lr}\text { Xe } & -0.0000000 \\ \text { F } & 1.3874309 \\ \text { F } & -1.3874309 \\ \text { F } & 1.3874309 \\ \text { F } & -1.3874309\end{array}$
0.0000000
0.0000000
$-1.3874309$
$-0.0000000$
1. 3874309
0.0000000
1. 3874309
0.0000000
$-1.3874309$
0.0000000

$\begin{array}{lr}\text { Xe } & -0.0000000 \\ \text { F } & -0.0000000 \\ \text { F } & 0.0000000 \\ \text { F } & 0.0000000 \\ \text { F } & 0.0000000 \\ \text { F } & 1.9455789 \\ \text { F } & -1.9455789\end{array}$
0.0000000
$-0.0000000$
1. 9455789
$-1.9455789$
$-0.0000000$
0.0000000
0.0000000
1. 9455789
0.0000000
$-1.9455789$
0.0000000
$-0.0000000$
$-0.0000000$
$-0.0000000$

\section{Optimized Structures at the LC- $\omega P B E$ Level}

The Cartesian coordinates of the structures are listed in Ångström.

$\begin{array}{lrrr}\text { Xe } & -0.0000000 & -0.0000000 & -0.0000000 \\ \text { F } & 0.0000000 & 0.0000000 & 1.9658891 \\ \text { F } & 0.0000000 & 0.0000000 & -1.9658891 \\ & & & \\ & & & \\ \text { Xe } & -0.0000000 & 0.0000000 & 0.0000000 \\ F & 1.3619215 & -1.3619215 & 0.0000000 \\ F & -1.3619215 & 1.3619215 & 0.0000000 \\ F & 1.3619215 & 1.3619215 & 0.0000000 \\ F & -1.3619215 & -1.3619215 & 0.0000000 \\ & & & \\ & & & \\ \text { Xe } & -0.0000000 & -0.0000000 & -0.0000000 \\ F & -0.0000000 & 1.9116172 & 0.0000000 \\ F & 0.0000000 & -1.9116172 & 0.0000000 \\ F & 0.0000000 & 0.0000000 & 1.9116172 \\ F & 0.0000000 & 0.0000000 & -1.9116172 \\ F & 1.9116172 & 0.0000000 & -0.0000000 \\ F & -1.9116172 & -0.0000000 & 0.0000000\end{array}$


12. Optimized Structures at the $\omega B 97 X-D$ Level

The Cartesian coordinates of the structures are listed in Ångström.

$\begin{array}{lrrr}\text { Xe } & -0.0000000 & -0.0000000 & 0.0000000 \\ \text { F } & 0.0000000 & 0.0000000 & 1.9810961 \\ \text { F } & 0.0000000 & -0.0000000 & -1.9810961 \\ & & & \\ & & & \\ \text { Xe } & -0.0000000 & 0.0000000 & 0.0000000 \\ \text { F } & 1.3719569 & -1.3719569 & 0.0000000 \\ \text { F } & -1.3719569 & 1.3719569 & -0.0000000 \\ \text { F } & 1.3719569 & 1.3719569 & 0.0000000 \\ \text { F } & -1.3719569 & -1.3719569 & 0.0000000 \\ & & & \\ & & & \\ \text { Xe } & 0.0000000 & 0.0000000 & 0.0000000 \\ \text { F } & 0.0000000 & 1.9243535 & -0.0000000 \\ \text { F } & -0.0000000 & -1.9243535 & -0.0000000 \\ \text { F } & -0.0000000 & 0.0000000 & 1.9243535 \\ \text { F } & -0.0000000 & 0.0000000 & -1.9243535 \\ \text { F } & 1.9243535 & -0.0000000 & -0.0000000 \\ \text { F } & -1.9243535 & 0.0000000 & 0.0000000\end{array}$

\section{Optimized Structures at the CAM-B3LYP Level}

The Cartesian coordinates of the structures are listed in Ångström.

$\begin{array}{lrrr}\text { Xe } & -0.0000000 & 0.0000000 & 0.0000000 \\ \text { F } & 0.0000000 & 0.0000000 & 1.9810858 \\ \text { F } & 0.0000000 & 0.0000000 & -1.9810858 \\ & & & \\ \text { Xe } & -0.0000000 & 0.0000000 & -0.0000000 \\ \text { F } & 1.3718215 & -1.3718215 & 0.0000000 \\ F & -1.3718215 & 1.3718215 & 0.0000000 \\ F & 1.3718215 & 1.3718215 & 0.0000000 \\ F & -1.3718215 & -1.3718215 & 0.0000000 \\ & & & \\ & & & \\ \text { Xe } & 0.0000000 & 0.0000000 & 0.0000000 \\ F & 0.0000000 & 1.9232569 & 0.0000000 \\ F & -0.0000000 & -1.9232569 & -0.0000000 \\ \text { F } & -0.0000000 & 0.0000000 & 1.9232569 \\ F & -0.0000000 & 0.0000000 & -1.9232569 \\ F & 1.9232569 & -0.0000000 & 0.0000000 \\ \text { F } & -1.9232569 & 0.0000000 & 0.0000000\end{array}$


14. Optimized Structures at the CAM-QTP-00 Level

The Cartesian coordinates of the structures are listed in Ångström.

$\begin{array}{lrrr}\text { Xe } & -0.0000000 & 0.0000000 & -0.0000000 \\ \text { F } & 0.0000000 & -0.0000000 & 1.9413209 \\ \text { F } & 0.0000000 & -0.0000000 & -1.9413209 \\ & & & \\ & & & \\ \text { Xe } & -0.0000000 & 0.0000000 & -0.0000000 \\ \text { F } & 1.3440257 & -1.3440257 & 0.0000000 \\ \text { F } & -1.3440257 & 1.3440257 & -0.0000000 \\ \text { F } & 1.3440257 & 1.3440257 & -0.0000000 \\ \text { F } & -1.3440257 & -1.3440257 & 0.0000000 \\ & & & \\ & & & \\ \text { Xe } & -0.0000000 & -0.0000000 & -0.0000000 \\ \text { F } & -0.0000000 & 1.8884349 & -0.0000000 \\ \text { F } & 0.0000000 & -1.8884349 & -0.0000000 \\ \text { F } & 0.0000000 & 0.0000000 & 1.8884349 \\ F & 0.0000000 & 0.0000000 & -1.8884349 \\ \text { F } & 1.8884349 & 0.0000000 & -0.0000000 \\ \text { F } & -1.8884349 & -0.0000000 & -0.0000000\end{array}$

\section{Optimized Structures at the CAM-QTP-02 Level}

The Cartesian coordinates of the structures are listed in Ångström.

$\begin{array}{lrrr}\text { Xe } & -0.0000000 & -0.0000000 & 0.0000000 \\ \text { F } & 0.0000000 & -0.0000000 & 1.9516634 \\ \text { F } & 0.0000000 & -0.0000000 & -1.9516634 \\ & & & \\ & & & \\ \text { Xe } & -0.0000000 & 0.0000000 & -0.0000000 \\ \text { F } & 1.3513826 & -1.3513826 & 0.0000000 \\ F & -1.3513826 & 1.3513826 & 0.0000000 \\ F & 1.3513826 & 1.3513826 & 0.0000000 \\ F & -1.3513826 & -1.3513826 & 0.0000000 \\ & & & \\ & & & \\ \text { Xe } & 0.0000000 & 0.0000000 & -0.0000000 \\ F & -0.0000000 & 1.8970904 & 0.0000000 \\ F & 0.0000000 & -1.8970904 & -0.0000000 \\ F & -0.0000000 & -0.0000000 & 1.8970904 \\ F & -0.0000000 & -0.0000000 & -1.8970904 \\ F & 1.8970904 & 0.0000000 & 0.0000000 \\ F & -1.8970904 & -0.0000000 & 0.0000000\end{array}$


16. Optimized Structures at the LHO7t-SVWN Level

The Cartesian coordinates of the structures are listed in Ångström.

$\begin{array}{lrrr}\text { Xe } & -0.0000000 & 0.0000000 & -0.0000000 \\ \text { F } & 0.0000000 & 0.0000000 & 1.9936386 \\ \text { F } & 0.0000000 & 0.0000000 & -1.9936386 \\ & & & \\ & & & \\ \text { Xe } & -0.0000000 & 0.0000000 & 0.0000000 \\ \text { F } & 1.3808804 & -1.3808804 & 0.0000000 \\ \text { F } & -1.3808804 & 1.3808804 & 0.0000000 \\ \text { F } & 1.3808804 & 1.3808804 & 0.0000000 \\ \text { F } & -1.3808804 & -1.3808804 & -0.0000000 \\ & & & \\ & & & \\ \text { Xe } & 0.0000000 & 0.0000000 & -0.0000000 \\ \text { F } & -0.0000000 & 1.9350471 & 0.0000000 \\ \text { F } & 0.0000000 & -1.9350471 & -0.0000000 \\ \text { F } & 0.0000000 & -0.0000000 & 1.9350471 \\ \text { F } & 0.0000000 & -0.0000000 & -1.9350471 \\ \text { F } & 1.9350471 & 0.0000000 & 0.0000000 \\ \text { F } & -1.9350471 & -0.0000000 & -0.0000000\end{array}$

17. Optimized Structures at the LH12ct-SsirPW92 Level

The Cartesian coordinates of the structures are listed in Ångström.

$\begin{array}{lrrr}\text { Xe } & -0.0000000 & 0.0000000 & 0.0000000 \\ \text { F } & 0.0000000 & 0.0000000 & 1.9877057 \\ \text { F } & 0.0000000 & -0.0000000 & -1.9877057 \\ & & & \\ \text { Xe } & -0.0000000 & 0.0000000 & 0.0000000 \\ \text { F } & 1.3766458 & -1.3766458 & 0.0000000 \\ F & -1.3766458 & 1.3766458 & 0.0000000 \\ F & 1.3766458 & 1.3766458 & 0.0000000 \\ F & -1.3766458 & -1.3766458 & -0.0000000 \\ & & & \\ & & & \\ \text { Xe } & -0.0000000 & 0.0000000 & -0.0000000 \\ \text { F } & 0.0000000 & 1.9295785 & 0.0000000 \\ F & -0.0000000 & -1.9295785 & 0.0000000 \\ \text { F } & 0.0000000 & -0.0000000 & 1.9295785 \\ F & 0.0000000 & -0.0000000 & -1.9295785 \\ F & 1.9295785 & -0.0000000 & 0.0000000 \\ \text { F } & -1.9295785 & 0.0000000 & 0.0000000\end{array}$


18. Optimized Structures at the LH14t-calPBE Level

The Cartesian coordinates of the structures are listed in Ångström.

$\begin{array}{lrrr}\text { Xe } & -0.0000000 & 0.0000000 & 0.0000000 \\ \text { F } & 0.0000000 & 0.0000000 & 1.9892774 \\ \text { F } & 0.0000000 & 0.0000000 & -1.9892774 \\ & & & \\ & & & \\ \text { Xe } & -0.0000000 & 0.0000000 & 0.0000000 \\ F & 1.3783111 & -1.3783111 & -0.0000000 \\ F & -1.3783111 & 1.3783111 & -0.0000000 \\ F & 1.3783111 & 1.3783111 & 0.0000000 \\ F & -1.3783111 & -1.3783111 & -0.0000000 \\ & & & \\ & & & \\ \text { Xe } & -0.0000000 & -0.0000000 & 0.0000000 \\ F & 0.0000000 & 1.9320329 & -0.0000000 \\ F & -0.0000000 & -1.9320329 & 0.0000000 \\ F & -0.0000000 & 0.0000000 & 1.9320329 \\ F & -0.0000000 & 0.0000000 & -1.9320329 \\ F & 1.9320329 & -0.0000000 & -0.0000000 \\ F & -1.9320329 & 0.0000000 & 0.0000000\end{array}$

19. Optimized Structures at the LH2Ot Level

The Cartesian coordinates of the structures are listed in Ångström.

$\begin{array}{lrrr}\text { Xe } & -0.0000000 & 0.0000000 & -0.0000000 \\ \text { F } & 0.0000000 & 0.0000000 & 1.9758435 \\ \text { F } & 0.0000000 & 0.0000000 & -1.9758435 \\ & & & \\ & & & \\ \text { Xe } & -0.0000000 & 0.0000000 & 0.0000000 \\ \text { F } & 1.3689255 & -1.3689255 & 0.0000000 \\ F & -1.3689255 & 1.3689255 & -0.0000000 \\ F & 1.3689255 & 1.3689255 & -0.0000000 \\ F & -1.3689255 & -1.3689255 & 0.0000000 \\ & & & \\ & & & \\ \text { Xe } & 0.0000000 & 0.0000000 & 0.0000000 \\ F & -0.0000000 & 1.9197949 & -0.0000000 \\ F & 0.0000000 & -1.9197949 & 0.0000000 \\ F & -0.0000000 & -0.0000000 & 1.9197949 \\ F & -0.0000000 & -0.0000000 & -1.9197949 \\ F & 1.9197949 & 0.0000000 & -0.0000000 \\ F & -1.9197949 & -0.0000000 & 0.0000000\end{array}$


20. Optimized Structures at the MPSTS Level

The Cartesian coordinates of the structures are listed in Ångström.

$\begin{array}{lrrr}\text { Xe } & -0.0000000 & -0.0000000 & -0.0000000 \\ \text { F } & 0.0000000 & 0.0000000 & 2.0011491 \\ \text { F } & 0.0000000 & 0.0000000 & -2.0011491 \\ & & & \\ & & & \\ \text { Xe } & -0.0000000 & 0.0000000 & 0.0000000 \\ \text { F } & 1.3872687 & -1.3872687 & -0.0000000 \\ \text { F } & -1.3872687 & 1.3872687 & 0.0000000 \\ \text { F } & 1.3872687 & 1.3872687 & -0.0000000 \\ \text { F } & -1.3872687 & -1.3872687 & -0.0000000 \\ & & & \\ & & & \\ \text { Xe } & -0.0000000 & -0.0000000 & 0.0000000 \\ F & 0.0000000 & 1.9455314 & -0.0000000 \\ F & -0.0000000 & -1.9455314 & 0.0000000 \\ F & -0.0000000 & -0.0000000 & 1.9455314 \\ F & 0.0000000 & -0.0000000 & -1.9455314 \\ F & 1.9455314 & -0.0000000 & 0.0000000 \\ F & -1.9455314 & 0.0000000 & 0.0000000\end{array}$

\section{Optimized Structures at the LHJ14 Level}

The Cartesian coordinates of the structures are listed in Ångström.

$\begin{array}{lrrr}\text { Xe } & -0.0000000 & 0.0000000 & 0.0000000 \\ \text { F } & 0.0000000 & 0.0000000 & 1.9915752 \\ \text { F } & 0.0000000 & 0.0000000 & -1.9915752 \\ & & & \\ & & & \\ \text { Xe } & -0.0000000 & 0.0000000 & -0.0000000 \\ \text { F } & 1.3803182 & -1.3803182 & 0.0000000 \\ F & -1.3803182 & 1.3803182 & 0.0000000 \\ F & 1.3803182 & 1.3803182 & 0.0000000 \\ F & -1.3803182 & -1.3803182 & -0.0000000 \\ & & & \\ & & & \\ \text { Xe } & -0.0000000 & -0.0000000 & -0.0000000 \\ F & -0.0000000 & 1.9348790 & 0.0000000 \\ F & 0.0000000 & -1.9348790 & -0.0000000 \\ F & 0.0000000 & -0.0000000 & 1.9348790 \\ F & 0.0000000 & -0.0000000 & -1.9348790 \\ F & 1.9348790 & 0.0000000 & -0.0000000 \\ F & -1.9348790 & -0.0000000 & -0.0000000\end{array}$




\section{E. NMR Shieldings, Shifts and Timings of Tungsten Compounds}

Tungsten compounds were studied with the same computational methods as in Sec. [S5C. Spin-orbit coupling is of minor importance for the respective shifts [110]. The structures were optimized and are listed after the NMR results. Geometries were considered converged when the energy change between optimization steps falls below $10^{-6} \mathrm{E}_{\mathrm{h}}$. In addition, the grid dependence and the effect on the wall time were studied. The CAM-QTP-00, CAM-QTP-02, and the current-dependent functionals are not considered in the grid dependence and the wall time studies as the aim of the studies is to compare the computational effort of conventional and local hybrid functionals. Point group symmetry is exploited [43]. The resolution of the identity approximation to the Coulomb integrals (RI-J) [28, 29, 31, 45] and the seminumerical approximation for the left-hand side of the CPKS equations [91] were employed in selected calculations. In the latter, it is possible to use different grids for the numerical integration of the HF exchange and the DFT contribution with global hybrid functionals. We use a small grid (gridsize -1 ) for the HF exchange in these calculations. The NMR shieldings and shifts are reported in Tabs. S66 to S77 Timings in Tabs. S78 to S84 are measured on a single thread of a CPU of type Intel® Xeon ${ }^{\circledR}$ Gold $6212 \mathrm{U}$ CPU @ $2.40 \mathrm{GHz}$. The code was compiled with the Intel® Fortran Compiler 19.0.1.144. A core Hamiltonian guess is used to construct the initial orbitals for the SCF procedure. The local hybrid functionals lead to a considerably increased computation time in all cases.

\section{NMR Shieldings and Shifts}

TABLE S66. Comparison of ${ }^{183} \mathrm{~W}$ NMR shielding constants (in ppm) at various levels (gridsize 4a). LH07t, LH12ct, and LH14t refer to the functionals LH07t-SVWN, LH12ct-SsirPW92, and LH14t-calPBE, respectively.

\begin{tabular}{|c|c|c|c|c|c|c|c|c|c|c|c|c|c|c|c|c|c|c|}
\hline & $\mathrm{HF}$ & KT3 & BP86 & PBE & TPSS & BH\&HLYP & B3LYP & PBE0 & TPSSh & LC- $\omega$ PBE & $\omega \mathrm{B} 97 \mathrm{X}-\mathrm{D}$ & CAM-B3LYP & $\mathrm{LH} 07 \mathrm{t}$ & LH12ct & LH14t & LH20t & mPSTS & LHJ14 \\
\hline $\mathrm{WO}_{4}^{2-}$ & 1803 & 1020 & 937 & 943 & 1038 & 1378 & 1073 & 1193 & 1130 & 1352 & 1224 & 1228 & 1085 & 1127 & 1126 & 1203 & 1144 & 1080 \\
\hline $\mathrm{WO}_{3} \mathrm{~S}_{2}^{-}$ & 874 & 304 & 182 & 203 & 297 & 484 & 235 & 395 & 366 & 536 & 399 & 367 & 254 & 288 & 302 & 355 & 379 & 274 \\
\hline $\mathrm{WO}_{2} \mathrm{~S}_{2}^{2-}$ & -411 & -549 & -709 & -676 & -583 & -635 & -773 & -579 & -553 & -518 & -640 & -697 & -741 & -728 & -685 & -665 & -546 & -677 \\
\hline $\mathrm{WOS}_{3}^{2-}$ & -1995 & -1476 & -1676 & -1632 & -1542 & -1900 & -1880 & -1660 & -1561 & -1719 & -1818 & -1879 & -1828 & -1845 & -1763 & -1785 & -1563 & -1709 \\
\hline $\mathrm{WS}_{4}^{2-}$ & -3787 & -2464 & -2710 & -2656 & -2569 & 3282 & -3074 & -2829 & -2645 & -3023 & -3103 & -3155 & -2993 & -3044 & -2919 & -2984 & -2658 & -2805 \\
\hline $\mathrm{WF}_{6}$ & 3608 & 1621 & 1508 & 1517 & 1655 & 2634 & 1937 & 2106 & 1877 & 2319 & 2175 & 2207 & 1942 & 2068 & 1996 & 2200 & 1902 & 1863 \\
\hline $\mathrm{WCl}_{6}$ & -444 & -1042 & -1374 & -1278 & -1171 & -1072 & -1486 & -990 & -1072 & -646 & -1039 & -1170 & -1343 & -1280 & -1238 & -1109 & -1089 & -1087 \\
\hline $\mathrm{W}(\mathrm{CO})_{6}$ & 4855 & 4839 & 4805 & 4859 & 4904 & 4974 & 4826 & 4859 & 4977 & 5144 & 4960 & 4955 & 5042 & 5102 & 5039 & 5066 & 4952 & 4926 \\
\hline
\end{tabular}

TABLE S67. Comparison of ${ }^{183} \mathrm{~W}$ NMR shifts (in ppm) at various levels (gridsize 4a). Experimental results are taken from Refs. 111 112 113 , and 114

\begin{tabular}{|c|c|c|c|c|c|c|c|c|c|c|c|c|c|c|c|c|c|c|c|}
\hline & $\mathrm{HF}$ & KT3 & BP86 & PBE & TPSS & BH\&HLYP & B3LYP & PBE0 & TPSSh & LC- $\omega$ PBE & $\omega \mathrm{B} 97 \mathrm{X}-\mathrm{D}$ & CAM-B3LYP & LH07t & LH12ct & LH14t & LH20t & mPSTS & LHJ14 & Exp. \\
\hline $\mathrm{S}^{-}$ & 929 & 716 & 755 & 741 & 740 & 894 & 838 & 798 & 764 & & 825 & 862 & 831 & 839 & 824 & 848 & 766 & 805 & 841 \\
\hline $\mathrm{WO}_{2} \mathrm{~S}_{2}^{2-}$ & 2214 & 1569 & 1646 & 1619 & 1621 & 2014 & 1846 & 1772 & 1683 & 1870 & 1863 & 1925 & 1826 & 1855 & 1811 & 1869 & 1690 & 1757 & 1787 \\
\hline $\mathrm{WOS}_{3}^{2-}$ & 3798 & 2496 & 2613 & 2576 & 2580 & 3278 & 2953 & 2853 & 2692 & 3071 & 3042 & 3107 & 2913 & 2972 & 2889 & 2988 & 2707 & 2789 & 2760 \\
\hline $\mathrm{WS}_{4}^{2-}$ & 5590 & 3485 & 3647 & 3599 & 3607 & 4660 & 4147 & 4022 & 3775 & 4375 & 4327 & 4384 & 4078 & 4171 & 4044 & 4187 & 3802 & 3885 & 3769 \\
\hline $\mathrm{WF}_{6}^{4}$ & -1805 & -601 & -571 & -574 & -617 & -1256 & -864 & -912 & -747 & -967 & -951 & -979 & -857 & -941 & -870 & -997 & -758 & -784 & -1121 \\
\hline $\mathrm{WCl}_{6}$ & 2247 & 2062 & 2311 & 2221 & 2209 & 2450 & 2559 & 2183 & 2203 & 1998 & 2263 & 2398 & 2428 & 2408 & 2364 & 2312 & 2233 & 2166 & 218 \\
\hline $\mathrm{W}(\mathrm{CO})_{6}$ & -3052 & -3818 & -3868 & -3916 & -3866 & -3959 & -3753 & -3666 & -3846 & -3971 & -3737 & -3727 & -3957 & -3975 & -3913 & -3862 & -3808 & -3846 & -344 \\
\hline
\end{tabular}

TABLE S68. Statistical evaluation of various computational methods with respect to the experimental findings. MAE, MSD, and STD denote the mean absolute error, mean signed error, and the standard deviation (all in ppm) regarding the latter.

\begin{tabular}{|c|c|c|c|c|c|c|c|c|c|c|c|c|c|c|c|c|c|c|}
\hline & $\mathrm{HF}$ & KT3 & BP86 & PBE & TPSS & BH\&HLYP & B3LYP & PBE0 & TPSSh & LC- $\omega$ PBE & $\omega \mathrm{B} 97 \mathrm{X}-\mathrm{D}$ & CAM-B3LYP & LH07t & LH12ct & LH14t & LH20t & mPSTS & LHJ14 \\
\hline MAE & 646 & 272 & 228 & 240 & 223 & 320 & 225 & 119 & 150 & 244 & 211 & 252 & 219 & 231 & 192 & 201 & 148 & 137 \\
\hline Max. Deviation 1 & 1821 & 520 & 550 & 891 & 547 & 504 & 378 & 253 & 400 & 606 & 558 & 615 & 511 & 529 & 467 & 418 & 363 & 400 \\
\hline MSD & 450 & -123 & -34 & -72 & -71 & 239 & 136 & 40 & -35 & 86 & 123 & 171 & 70 & 80 & 54 & 82 & -20 & 0 \\
\hline STD & 795 & 297 & 304 & 313 & 286 & 372 & 244 & 161 & 229 & 315 & 262 & 277 & 282 & 297 & 254 & 256 & 217 & 220 \\
\hline
\end{tabular}


TABLE S69. Comparison of ${ }^{183} \mathrm{~W}$ NMR shielding constants (in ppm) at various levels (gridsize 4a). LH07t, LH12ct, and LH14t refer to the functionals LH07t-SVWN, LH12ct-SsirPW92, and LH14t-calPBE, respectively.

\begin{tabular}{|c|c|c|c|c|c|c|c|c|c|c|}
\hline & CAM-QTP-00 & CAM-QTP-02 & cTPSS & cTPSSh & cLH07t & cLH12ct & $\mathrm{cLH} 14 \mathrm{t}$ & $\mathrm{cLH} 20 \mathrm{t}$ & cmPSTS & cLHJ14 \\
\hline $\mathrm{WO}_{4}^{2-}$ & 1515 & 1407 & 1024 & 1118 & 1094 & 1139 & 1130 & 1211 & 1132 & 1083 \\
\hline $\mathrm{WO}_{3} \mathrm{~S}_{2}^{-}$ & 611 & 521 & 285 & 355 & 265 & 302 & 306 & 363 & 367 & 278 \\
\hline $\mathrm{WO}_{2} \mathrm{~S}_{2}^{2-}$ & -545 & -600 & -597 & -565 & -729 & -713 & -680 & -655 & -558 & -668 \\
\hline $\mathrm{WOS}_{3}^{2-}$ & -1869 & -1866 & -1559 & -1578 & -1817 & -1830 & -1758 & -1770 & -1579 & -1691 \\
\hline $\mathrm{WS}_{4}^{2-}$ & -3320 & -3242 & -2592 & -2667 & -2983 & -3029 & -2912 & -2963 & -2681 & -2773 \\
\hline $\mathrm{WF}_{6}^{4}$ & 2869 & 2558 & 1652 & 1875 & 1953 & 2081 & 2000 & 2202 & 1900 & 1854 \\
\hline $\mathrm{WCl}_{6}$ & -729 & -800 & -1178 & -1078 & -1332 & -1269 & -1232 & -1099 & -1095 & -1075 \\
\hline $\mathrm{W}(\mathrm{CO})_{6}$ & 5080 & 5121 & 4876 & 4950 & 5012 & 5072 & 5031 & 5074 & 4926 & 4981 \\
\hline
\end{tabular}

TABLE S70. Comparison of ${ }^{183} \mathrm{~W}$ NMR shifts (in ppm) at various levels (gridsize 4a). Experimental results are taken from Refs. 111 112 113 , and 114

\begin{tabular}{|c|c|c|c|c|c|c|c|c|c|c|c|}
\hline & CAM-QTP-00 & CAM-QTP-02 & cTPSS & cTPSSh & $\mathrm{cLH} 07 \mathrm{t}$ & $\mathrm{cLH} 12 \mathrm{ct}$ & cLH14t & cLH20t & cmPSTS & cLHJ14 & Exp. \\
\hline $\mathrm{WO}_{3} \mathrm{~S}_{2}^{-}$ & 904 & 885 & 740 & 763 & 829 & 837 & 823 & 847 & 765 & 805 & 841 \\
\hline $\mathrm{WO}_{2} \mathrm{~S}_{2}^{2-}$ & 2060 & 2007 & 1621 & 1684 & 1823 & 1852 & 1809 & 1865 & 1690 & 1751 & 1787 \\
\hline $\mathrm{WOS}_{3}^{2-}$ & 3383 & 3273 & 1621 & 1684 & 1823 & 1852 & 1809 & 1865 & 1690 & 1751 & 1787 \\
\hline $\mathrm{WS}_{4}^{2-}$ & 4835 & 4649 & 3616 & 3786 & 4077 & 4168 & 4042 & 4173 & 3813 & 3856 & 3769 \\
\hline $\mathrm{WF}_{6}^{4}$ & -1354 & -1152 & -628 & -757 & -859 & -941 & -870 & -992 & -768 & -770 & -1121 \\
\hline $\mathrm{WCl}_{6}$ & 2244 & 2207 & 2202 & 2196 & 2426 & 2408 & 2362 & 2309 & 2227 & 2158 & 2181 \\
\hline $\mathrm{W}(\mathrm{CO})_{6}$ & -3566 & -3714 & -3852 & -3832 & -3918 & -3932 & -3902 & -3863 & -3794 & -3898 & -3446 \\
\hline
\end{tabular}

TABLE S71. Statistical evaluation of various computational methods with respect to the experimental findings. MAE, MSD, and STD denote the mean absolute error, mean signed error, and the standard deviation (all in ppm) regarding the latter.

\begin{tabular}{|c|c|c|c|c|c|c|c|c|c|c|}
\hline & CAM-QTP-00 & CAM-QTP-02 & cTPSS & cTPSSh & $\mathrm{cLH} 07 \mathrm{t}$ & $\mathrm{cLH} 12 \mathrm{ct}$ & cLH14t & $\mathrm{cLH} 20 \mathrm{t}$ & cmPSTS & cLHJ14 \\
\hline MAE & 349 & 282 & 185 & 107 & 169 & 181 & 145 & 161 & 111 & 91 \\
\hline Max. Deviation & 1066 & 880 & 493 & 364 & 308 & 399 & 273 & 404 & 353 & 351 \\
\hline MSD & 248 & 198 & -70 & -34 & 74 & 84 & 54 & 78 & -18 & -14 \\
\hline STD & 456 & 385 & 279 & 222 & 268 & 282 & 250 & 252 & 210 & 237 \\
\hline
\end{tabular}


TABLE S72. Comparison of ${ }^{183} \mathrm{~W}$ NMR shielding constants (in ppm) at various levels (gridsize 2a).

\begin{tabular}{|c|c|c|c|c|c|c|c|c|c|c|c|c|c|c|c|c|c|c|}
\hline & HF & KT3 & BP86 & PBE & TPSS & BH\&HLYP & B3LYP & PBE0 & TPSSh & LC- $\omega$ PBE & $\omega \mathrm{B} 97 \mathrm{X}-\mathrm{D}$ & CAM-B3LYP & $\mathrm{LH} 07 \mathrm{t}$ & LH12ct & LH14t & LH20t & mPSTS & LHJ14 \\
\hline $\mathrm{WO}_{4}^{2-}$ & 1803 & 1020 & 937 & 943 & 1038 & 1378 & 1073 & 1193 & 1130 & 1352 & 1223 & 1228 & 1085 & 1127 & 1125 & 1206 & 1144 & 1079 \\
\hline $\mathrm{WO}_{3} \mathrm{~S}^{2-}$ & 874 & 304 & 182 & 203 & 297 & 484 & 235 & 396 & 367 & 536 & 399 & 367 & 254 & 288 & 302 & 357 & 379 & 274 \\
\hline $\mathrm{WO}_{2} \mathrm{~S}_{2}^{2-}$ & -411 & -549 & -709 & -676 & -583 & -635 & -773 & -579 & -553 & -518 & -641 & -697 & -741 & -728 & -685 & -664 & -546 & -677 \\
\hline $\mathrm{WOS}_{3}^{2-}$ & -1995 & -1476 & -1676 & -676 & -1542 & -1900 & -1880 & -1660 & -1561 & -1719 & -1822 & -1879 & -1828 & -1845 & -1764 & -1784 & -1563 & -1709 \\
\hline $\mathrm{WS}_{4}^{2-}$ & -3787 & -2463 & -2709 & -2656 & -2568 & -3281 & -3073 & -2828 & -2644 & -3022 & -3106 & -3155 & -2992 & -3043 & -2919 & -2982 & -2657 & -2805 \\
\hline $\mathrm{WF}_{6}^{4}$ & 3608 & 1622 & 1508 & 1518 & 1657 & 2634 & 1937 & 2106 & 1879 & 2321 & 2174 & 2207 & 1942 & 2069 & 1997 & 2210 & 1904 & 1865 \\
\hline $\mathrm{WCl}_{6}$ & -444 & -1040 & -1374 & -1277 & -1169 & -1072 & -1486 & -989 & -1070 & -645 & -1038 & -1170 & -1342 & -1280 & -1240 & -1101 & -1087 & -1088 \\
\hline $\mathrm{W}(\mathrm{CO})_{6}$ & 4855 & 4841 & 4808 & 4862 & 4906 & 4975 & 4829 & 4862 & 4979 & 5145 & 4964 & 4957 & 5045 & 5105 & 5041 & 5066 & 4954 & 4927 \\
\hline
\end{tabular}

TABLE S73. Comparison of ${ }^{183} \mathrm{~W}$ NMR shifts (in ppm) at various levels (gridsize 2a).

\begin{tabular}{|c|c|c|c|c|c|c|c|c|c|c|c|c|c|c|c|c|c|c|c|}
\hline & $\mathrm{HF}$ & KT3 & BP86 & PBE & TPSS & BH\&HLYP & B3LYP & PBE0 & TPSSh & LC- $\omega$ PBE & $\omega \mathrm{B} 97 \mathrm{X}-\mathrm{D}$ & CAM-B3LYP & LH07t & LH12ct & LH14t & LH20t & mPSTS & LHJ14 & Exp. \\
\hline $\mathrm{WO}_{3} \mathrm{~S}^{2-}$ & 929 & 716 & 755 & 741 & 740 & 894 & 838 & 798 & 764 & 816 & 825 & 862 & 831 & 839 & 823 & 849 & 765 & 805 & 84 \\
\hline $\mathrm{WO}_{2} \mathrm{~S}_{2}^{2-}$ & 2214 & 1569 & 1646 & 1619 & 1620 & 2014 & 1846 & 1772 & 1683 & 1870 & 1864 & 1925 & 1826 & 1855 & 1810 & 1870 & 1690 & 1757 & 1787 \\
\hline & 3798 & 2496 & 2613 & 1619 & 2580 & 3278 & 2953 & 2853 & 2692 & 3071 & 3045 & 3107 & 2913 & 2972 & 2889 & 2990 & 2707 & 2789 & 2760 \\
\hline W & 5590 & 3484 & 3646 & 3599 & 3606 & 4659 & 4146 & 4022 & 3774 & 4375 & 4329 & 4383 & 4078 & 4170 & 4045 & 4188 & 3801 & 3884 & 3769 \\
\hline & -1805 & -602 & -571 & -575 & -619 & -1256 & -864 & -913 & -749 & -9679 & -951 & -979 & -857 & -941 & -872 & -1004 & -760 & -786 & -112 \\
\hline & 2247 & 2061 & 2311 & 2220 & 2207 & 2450 & 2559 & 2183 & 2201 & 1997 & 2261 & 2398 & 2427 & 2407 & 2365 & 2307 & 2231 & 2168 & 218 \\
\hline $\mathrm{W}(\mathrm{CO})_{6}$ & -3052 & -3821 & -3871 & -3918 & -3868 & -3597 & -3756 & -3668 & -3848 & -3793 & -3741 & -3729 & -3960 & -3977 & -3916 & -3861 & -3810 & -3848 & -344 \\
\hline
\end{tabular}


TABLE S74. Comparison of ${ }^{183} \mathrm{~W}$ NMR shielding constants (in ppm) at various levels (gridsize 2a and seminumerical exchange for CPKS).

\begin{tabular}{|c|c|c|c|c|c|c|c|c|c|c|c|c|c|c|c|c|c|c|}
\hline & $\mathrm{HF}$ & KT3 & BP86 & PBE & TPSS & BH\&HLYP & B3LYP & PBE0 & TPSSh & LC- $\omega$ PBE & $\omega \mathrm{B} 97 \mathrm{X}-\mathrm{D}$ & CAM-B3LYP & LH07t & LH12ct & LH14t & LH20t & mPSTS & LHJ14 \\
\hline $\mathrm{WO}_{4}^{2-}$ & 1804 & 1020 & 937 & 943 & 1038 & 1073 & 1378 & 1193 & 1130 & 1351 & 1222 & 1227 & 1085 & 1127 & 1125 & 1206 & 1144 & 1079 \\
\hline $\mathrm{WO}_{3} \mathrm{~S}^{2-}$ & 875 & 304 & 182 & 203 & 297 & 484 & 235 & 396 & 367 & 537 & 399 & 367 & 254 & 288 & 302 & 357 & 379 & 274 \\
\hline $\mathrm{WO}_{2} \mathrm{~S}_{2}^{2-}$ & -410 & -549 & -709 & -676 & -583 & -635 & -773 & -579 & -553 & -517 & -640 & -696 & 741 & -728 & -685 & -664 & -546 & -677 \\
\hline $\mathrm{WOS}_{3}^{2-}$ & -1994 & -1476 & -1676 & -676 & -1542 & -1899 & -1880 & -1660 & -1561 & -1718 & -1821 & -1879 & -1828 & -1845 & -1764 & -1784 & -1563 & -1709 \\
\hline $\mathrm{WS}_{4}^{2-}$ & -3786 & -2463 & -2709 & -2656 & -2568 & -3280 & -3072 & -2828 & -2644 & -3022 & -3105 & -3154 & -2992 & -3043 & -2919 & -2982 & -2657 & -2805 \\
\hline $\mathrm{WF}_{6}^{4}$ & 3606 & 1622 & 1508 & 1518 & 1657 & 2632 & 1936 & 2105 & 1879 & 2316 & 2170 & 2204 & 1942 & 2069 & 1997 & 2210 & 1904 & 1865 \\
\hline $\mathrm{WCl}_{6}$ & -446 & -1040 & -1374 & -1277 & -1169 & -1073 & -1486 & -990 & -1071 & -647 & -1040 & -1171 & -1342 & -1280 & -1240 & -1101 & -1087 & -1088 \\
\hline $\mathrm{W}(\mathrm{CO})_{6}$ & 4843 & 4841 & 4808 & 4862 & 4906 & 4972 & 4827 & 4862 & 4978 & 5139 & 4959 & 4953 & 5045 & 5105 & 5041 & 5066 & 4954 & 4927 \\
\hline
\end{tabular}

TABLE S75. Comparison of ${ }^{183} \mathrm{~W}$ NMR shifts (in ppm) at various levels (gridsize 2a and seminumerical exchange for CPKS).

\begin{tabular}{|c|c|c|c|c|c|c|c|c|c|c|c|c|c|c|c|c|c|c|c|}
\hline & $\mathrm{HF}$ & KT3 & BP86 & PBE & TPSS & BH\&HLYP & B3LYP & PBE0 & TPSSh & $L C-\omega \mathrm{PBE}$ & $\omega \mathrm{B} 97 \mathrm{X}-\mathrm{D}$ & CAM-B3LYP & LH07t & LH12ct & LH14t & LH20t & mPSTS & LHJ14 & Exp. \\
\hline $\mathrm{WO}_{3} \mathrm{~S}^{2-}$ & 929 & 716 & 755 & 741 & 740 & 893 & 838 & 797 & 763 & 814 & 823 & 860 & 831 & 839 & 823 & 849 & 765 & 805 & 841 \\
\hline $\mathrm{WO}_{2} \mathrm{~S}_{2}^{2-}$ & 2214 & 1569 & 1646 & 1619 & 1620 & 2013 & 1846 & 1772 & 1683 & 1868 & 1862 & 1924 & 1826 & 1855 & 1810 & 1870 & 1690 & 1757 & 1787 \\
\hline $\mathrm{WOS}_{3}^{2-}$ & 3798 & 2496 & 2613 & 2576 & 2580 & 3277 & 2953 & 2853 & 2691 & 3069 & 3043 & 3106 & 2913 & 2972 & 2889 & 2990 & 2707 & 2789 & 2760 \\
\hline $\mathrm{WS}_{4}^{2-}$ & 5590 & 3484 & 3646 & 3599 & 3606 & 4657 & 4145 & 4021 & 3774 & 4373 & 4327 & 4381 & 4078 & 4170 & 4045 & 4188 & 3801 & 3884 & 3769 \\
\hline $\mathrm{WF}_{6}$ & -1802 & -602 & -571 & -575 & -619 & -1254 & -863 & -913 & -749 & -965 & -948 & -977 & -857 & -941 & -872 & -1004 & -760 & -786 & -1121 \\
\hline $\mathrm{WCl}_{6}$ & 2250 & 2061 & 2311 & 2220 & 2207 & 2451 & 2559 & 2183 & 2201 & 1998 & 2262 & 2399 & 2427 & 2407 & 2365 & 2307 & 2231 & 2168 & 2181 \\
\hline $\mathrm{W}(\mathrm{CO})_{6}$ & -3039 & -3821 & -3871 & -3918 & -3868 & -3594 & -3755 & -3669 & -3848 & -3788 & -3737 & -3726 & -3960 & -3977 & -3916 & -3861 & -3810 & -3848 & -3446 \\
\hline
\end{tabular}


TABLE S76. Comparison of ${ }^{183} \mathrm{~W}$ NMR shielding constants (in ppm) at various levels (gridsize 2a, RI-J, and seminumerical exchange for CPKS).

\begin{tabular}{|c|c|c|c|c|c|c|c|c|c|c|c|c|c|c|c|c|c|c|}
\hline & $\mathrm{HF}$ & KT3 & BP86 & PBE & TPSS & BH\&HLYP & B3LYP & PBE0 & TPSSh & LC- $\omega$ PBE & $\omega \mathrm{B} 97 \mathrm{X}-\mathrm{D}$ & CAM-B3LYP & LH07t & LH12ct & LH14t & LH20t & mPSTS & LHJ14 \\
\hline $\mathrm{JO}_{4}^{2-}$ & 1805 & 1021 & 938 & 944 & 1038 & 1379 & 1074 & 1193 & 1131 & 1352 & 1223 & 1228 & 1086 & 1128 & 1126 & 1207 & 1145 & 1080 \\
\hline $\mathrm{WO}_{3} \mathrm{~S}^{2-}$ & 877 & 306 & 183 & 204 & 299 & 486 & 237 & 398 & 368 & 538 & 501 & 369 & 256 & 290 & 304 & 359 & 380 & 276 \\
\hline $\mathrm{WO}_{2} \mathrm{~S}_{2}^{2-}$ & -407 & -547 & -707 & -674 & -581 & -633 & -771 & -577 & -551 & -515 & -638 & -694 & -739 & -726 & -683 & -662 & -544 & -675 \\
\hline $\mathrm{WOS}_{3}^{2-}$ & -1991 & -1474 & -1674 & -1631 & -1540 & -1897 & -1878 & -1658 & -1559 & -1716 & -1819 & -1876 & -1826 & -1843 & -1762 & -1782 & -1561 & -1707 \\
\hline $\mathrm{WS}_{4}^{2-}$ & -3783 & -2462 & -2708 & -2654 & -2566 & -3277 & -3070 & -2826 & -2642 & -3020 & -3103 & -3152 & -2991 & -3041 & -2918 & -2980 & -2655 & -2803 \\
\hline $\mathrm{WF}_{6}^{4}$ & 3606 & 1622 & 1509 & 1518 & 1657 & 2632 & 1936 & 2106 & 1879 & 2317 & 2171 & 2204 & 1943 & 2069 & 1997 & 2210 & 1905 & 1866 \\
\hline $\mathrm{WCl}_{6}$ & -445 & -1040 & -1373 & -1276 & -1169 & -1072 & -1486 & -990 & -1070 & -647 & -1039 & -1171 & -1342 & -1279 & -1239 & -1101 & -1087 & -1088 \\
\hline $\mathrm{W}(\mathrm{CO})_{6}$ & 4843 & 4841 & 4808 & 4862 & 4906 & 4972 & 4827 & 4862 & 4978 & 5139 & 4959 & 4953 & 5045 & 5105 & 5041 & 5066 & 4954 & 4927 \\
\hline
\end{tabular}

TABLE S77. Comparison of ${ }^{183} \mathrm{~W}$ NMR shifts (in ppm) at various levels (gridsize 2a, RI-J, and seminumerical exchange for CPKS).

\begin{tabular}{|c|c|c|c|c|c|c|c|c|c|c|c|c|c|c|c|c|c|c|c|}
\hline & $\mathrm{HF}$ & KT3 & BP86 & PBE & TPSS & BH\&HLYP & B3LYP & PBE0 & TPSSh & $L C-\omega \mathrm{PBE}$ & $\omega \mathrm{B} 97 \mathrm{X}-\mathrm{D}$ & CAM-B3LYP & LH07t & LH12ct & LH14t & LH20t & mPSTS & LHJ14 & Exp. \\
\hline $\mathrm{O}_{3} \mathrm{~S}^{2-}$ & 928 & 716 & 755 & 740 & 739 & 893 & 837 & 795 & 763 & 814 & 822 & 860 & 831 & 839 & 823 & 848 & 765 & 804 & 841 \\
\hline $\mathrm{WO}_{2} \mathrm{~S}_{2}^{2-}$ & 2212 & 1569 & 1645 & 1618 & 1620 & 2012 & 1845 & 1770 & 1682 & 1867 & 1861 & 1923 & 1825 & 1854 & 1809 & 1869 & 1689 & 1756 & 1787 \\
\hline $\mathrm{WOS}_{3}^{2-}$ & 3796 & 2496 & 2612 & 2575 & 2579 & 3276 & 2952 & 2851 & 2690 & 3068 & 3042 & 3105 & 2913 & 2971 & 2888 & 2989 & 2706 & 2788 & 2760 \\
\hline $\mathrm{WS}_{4}^{2-}$ & 5588 & 3483 & 3646 & 3598 & 3605 & 4656 & 4144 & 4019 & 3773 & 4372 & 4326 & 4380 & 4077 & 4170 & 4044 & 4187 & 3800 & 3883 & 3769 \\
\hline $\mathrm{WF}_{6}$ & -1801 & -601 & -571 & -574 & -619 & -1253 & -863 & -913 & -748 & -965 & -947 & -976 & -856 & -941 & -871 & -1003 & -760 & -785 & -1121 \\
\hline $\mathrm{WCl}_{6}$ & 2250 & 2061 & 2311 & 2220 & 2207 & 2451 & 2560 & 2182 & 2201 & 1999 & 2263 & 2399 & 2428 & 2408 & 2366 & 2308 & 2232 & 2168 & 2181 \\
\hline $\mathrm{W}(\mathrm{CO})_{6}$ & -3037 & -3820 & -3870 & -3918 & -3868 & -3593 & -3754 & -3669 & -3847 & -3787 & -3736 & -3724 & -3959 & -3976 & -3915 & -3859 & -3809 & -3847 & -3446 \\
\hline
\end{tabular}




\section{Timings}

TABLE S78. Wall times of the full SCF procedure in seconds (gridsize 4a).

\begin{tabular}{|c|c|c|c|c|c|c|c|c|c|c|c|c|c|c|c|c|c|c|}
\hline & $\mathrm{HF}$ & KT3 & BP86 & PBE & TPSS & BH\&HLYP & B3LYP & PBE0 & TPSSh & LC- $\omega$ PBE & $\omega \mathrm{B} 97 \mathrm{X}-\mathrm{D}$ & CAM-B3LYP & LH07t & LH12ct & LH14t & LH20t & mPSTS & LHJ14 \\
\hline $\mathrm{WO}_{4}^{2-}$ & 61 & 78 & 52 & 58 & 62 & 60 & 83 & 81 & 78 & 105 & 97 & 103 & 153 & 146 & 131 & 127 & 123 & 138 \\
\hline $\mathrm{WO}_{3} \mathrm{~S}^{2-}$ & 381 & 481 & 363 & 393 & 398 & 425 & 398 & 314 & 403 & 598 & 682 & 645 & 1559 & 1457 & 1529 & 1427 & 1335 & 1499 \\
\hline $\mathrm{WO}_{2} \mathrm{~S}_{2}^{2-}$ & 409 & 533 & 426 & 474 & 439 & 462 & 446 & 424 & 501 & 935 & 837 & 753 & 1720 & 1764 & 1406 & 2087 & 1854 & 1650 \\
\hline $\mathrm{WOS}_{3}^{2-}$ & 482 & 689 & 481 & 388 & 499 & 479 & 457 & 502 & 570 & 844 & 837 & 796 & 1704 & 1855 & 1780 & 1722 & 1861 & 2201 \\
\hline $\mathrm{WS}_{4}^{2-}$ & 496 & 693 & 446 & 435 & 486 & 108 & 471 & 542 & 570 & 169 & 156 & 165 & 162 & 2175 & 1932 & 1891 & 1883 & 2291 \\
\hline $\mathrm{WF}_{6}^{4}$ & 67 & 62 & 53 & 54 & 46 & 71 & 67 & 72 & 68 & 99 & 100 & 102 & 124 & 112 & 109 & 110 & 111 & 118 \\
\hline $\mathrm{WCl}_{6}$ & 110 & 78 & 69 & 58 & 69 & 86 & 84 & 70 & 70 & 153 & 137 & 127 & 123 & 154 & 159 & 138 & 157 & 175 \\
\hline $\mathrm{W}(\mathrm{CO})_{6}$ & 198 & 195 & 193 & 185 & 207 & 180 & 202 & 189 & 179 & 306 & 286 & 314 & 302 & 350 & 361 & 380 & 404 & 339 \\
\hline TOTAL & 2203 & 2808 & 2083 & 2044 & 2206 & 1872 & 2207 & 2194 & 2438 & 3209 & 3132 & 3005 & 5846 & 8014 & 7407 & 7881 & 7726 & 8411 \\
\hline
\end{tabular}

TABLE S79. Wall times of the NMR shielding calculations in seconds (gridsize 4a).

\begin{tabular}{|c|c|c|c|c|c|c|c|c|c|c|c|c|c|c|c|c|c|c|}
\hline & $\mathrm{HF}$ & KT3 & BP86 & PBE & TPSS & BH\&HLYP & B3LYP & PBE0 & TPSSh & LC- $\omega$ PBE & $\omega \mathrm{B} 97 \mathrm{X}-\mathrm{D}$ & CAM-B3LYP & LH07t & LH12ct & LH14t & LH20t & mPSTS & LHJ14 \\
\hline $\mathrm{WO}_{4}^{2-}$ & 40 & 11 & 8 & 10 & 9 & 37 & 36 & 35 & 32 & 40 & 42 & 38 & 48 & 45 & 46 & 49 & 36 & 48 \\
\hline $\mathrm{WO}_{3} \mathrm{~S}^{2-}$ & 209 & 47 & 44 & 50 & 62 & 202 & 163 & 148 & 140 & 227 & 201 & 198 & 581 & 576 & 518 & 543 & 485 & 499 \\
\hline $\mathrm{WO}_{2} \mathrm{~S}_{2}^{2-}$ & 222 & 48 & 49 & 44 & 58 & 219 & 153 & 157 & 144 & 280 & 231 & 214 & 530 & 569 & 537 & 675 & 578 & 526 \\
\hline $\mathrm{WOS}_{3}^{2-}$ & 296 & 69 & 58 & 46 & 60 & 237 & 159 & 164 & 182 & 282 & 251 & 256 & 550 & 605 & 583 & 624 & 616 & 654 \\
\hline $\mathrm{WS}_{4}^{2-}$ & 312 & 64 & 47 & 52 & 61 & 49 & 179 & 194 & 183 & 56 & 56 & 49 & 58 & 757 & 675 & 730 & 619 & 704 \\
\hline $\mathrm{WF}_{6}$ & 55 & 13 & 13 & 13 & 11 & 42 & 36 & 40 & 36 & 45 & 47 & 43 & 47 & 47 & 47 & 50 & 46 & 48 \\
\hline $\mathrm{WCl}_{6}$ & 63 & 17 & 17 & 13 & 17 & 57 & 50 & 41 & 18 & 61 & 62 & 58 & 61 & 59 & 65 & 58 & 57 & 58 \\
\hline $\mathrm{W}(\mathrm{CO})_{6}$ & 157 & 44 & 39 & 39 & 45 & 140 & 95 & 40 & 81 & 136 & 138 & 142 & 121 & 141 & 135 & 138 & 127 & 131 \\
\hline Total & 1356 & 312 & 275 & 267 & 323 & 982 & 871 & 818 & 816 & 1127 & 1027 & 998 & 1996 & 2798 & 2606 & 2867 & 2563 & 2668 \\
\hline
\end{tabular}


TABLE S80. Wall times of the full SCF procedure in seconds (gridsize 2a).

\begin{tabular}{|c|c|c|c|c|c|c|c|c|c|c|c|c|c|c|c|c|c|c|}
\hline & $\mathrm{HF}$ & KT3 & BP86 & PBE & TPSS & BH\&HLYP & B3LYP & PBE0 & TPSSh & LC- $\omega$ PBE & $\omega \mathrm{B} 97 \mathrm{X}-\mathrm{D}$ & CAM-B3LYP & LH07t & LH12ct & LH14t & LH20t & mPSTS & LHJ14 \\
\hline $\mathrm{WO}_{4}^{2-}$ & 61 & 72 & 58 & 58 & 69 & 59 & 76 & 79 & 77 & 132 & 114 & 126 & 109 & 106 & 103 & 73 & 103 & 89 \\
\hline $\mathrm{WO}_{3} \mathrm{~S}^{2-}$ & 381 & 378 & 372 & 322 & 358 & 402 & 371 & 346 & 321 & 664 & 744 & 733 & 759 & 732 & 845 & 797 & 672 & 737 \\
\hline $\mathrm{WO}_{2} \mathrm{~S}_{2}^{2-}$ & 409 & 520 & 418 & 460 & 466 & 448 & 422 & 411 & 455 & 868 & 903 & 954 & 927 & 944 & 1062 & 1026 & 845 & 941 \\
\hline $\mathrm{WOS}_{3}^{2-}$ & 482 & 530 & 463 & 444 & 437 & 478 & 468 & 481 & 483 & 932 & 879 & 900 & 1003 & 993 & 945 & 857 & 887 & 1010 \\
\hline $\mathrm{WS}_{4}^{2-}$ & 496 & 87 & 97 & 80 & 82 & 112 & 100 & 109 & 100 & 210 & 176 & 196 & 119 & 133 & 130 & 134 & 122 & 142 \\
\hline $\mathrm{WF}_{6}$ & 67 & 55 & 52 & 54 & 51 & 68 & 65 & 71 & 66 & 124 & 119 & 113 & 86 & 80 & 78 & 78 & 73 & 82 \\
\hline $\mathrm{WCl}_{6}$ & 110 & 73 & 75 & 65 & 66 & 85 & 84 & 77 & 96 & 179 & 171 & 152 & 109 & 115 & 117 & 113 & 111 & 110 \\
\hline $\mathrm{W}(\mathrm{CO})_{6}$ & 198 & 179 & 192 & 183 & 192 & 178 & 202 & 183 & 203 & 374 & 349 & 349 & 246 & 233 & 275 & 256 & 276 & 259 \\
\hline Total & 2203 & 1893 & 1726 & 1666 & 1720 & 1830 & 1787 & 1756 & 1801 & 3484 & 3454 & 3523 & 3358 & 3335 & 3554 & 3333 & 3090 & 3368 \\
\hline
\end{tabular}

TABLE S81. Wall times of the NMR shielding calculations in seconds (gridsize 2a).

\begin{tabular}{|c|c|c|c|c|c|c|c|c|c|c|c|c|c|c|c|c|c|c|}
\hline & $\mathrm{HF}$ & KT3 & BP86 & PBE & TPSS & BH\&HLYP & B3LYP & PBE0 & TPSSh & LC- $\omega$ PBE & $\omega \mathrm{B} 97 \mathrm{X}-\mathrm{D}$ & CAM-B3LYP & LH07t & LH12ct & LH14t & LH20t & mPSTS & LHJ14 \\
\hline $\mathrm{WO}_{4}^{2-}$ & 40 & 10 & 10 & 10 & 11 & 37 & 32 & 35 & 32 & 50 & 51 & 47 & 25 & 26 & 27 & 22 & 25 & 25 \\
\hline $\mathrm{WO}_{3} \mathrm{~S}^{2-}$ & 209 & 40 & 40 & 47 & 52 & 199 & 157 & 147 & 133 & 223 & 205 & 225 & 216 & 199 & 220 & 220 & 195 & 207 \\
\hline $\mathrm{WO}_{2} \mathrm{~S}_{2}^{2-}$ & 222 & 43 & 40 & 51 & 49 & 215 & 151 & 150 & 135 & 283 & 241 & 262 & 214 & 233 & 224 & 240 & 203 & 216 \\
\hline $\mathrm{WOS}_{3}^{2-}$ & 296 & 53 & 52 & 55 & 54 & 233 & 160 & 163 & 146 & 329 & 285 & 284 & 231 & 250 & 239 & 259 & 223 & 271 \\
\hline $\mathrm{WS}_{4}^{2-}$ & 312 & 13 & 12 & 13 & 12 & 49 & 42 & 43 & 37 & 69 & 63 & 58 & 31 & 34 & 33 & 28 & 30 & 35 \\
\hline $\mathrm{WF}_{6}^{4}$ & 55 & 13 & 13 & 13 & 13 & 41 & 35 & 40 & 36 & 57 & 56 & 53 & 28 & 24 & 29 & 24 & 23 & 28 \\
\hline $\mathrm{WCl}_{6}$ & 63 & 16 & 16 & 16 & 16 & 56 & 50 & 46 & 45 & 72 & 73 & 68 & 38 & 38 & 39 & 32 & 36 & 38 \\
\hline $\mathrm{W}(\mathrm{CO})_{6}$ & 157 & 43 & 42 & 42 & 43 & 139 & 98 & 40 & 86 & 150 & 155 & 146 & 82 & 86 & 87 & 73 & 64 & 79 \\
\hline Total & 1356 & 230 & 226 & 247 & 249 & 969 & 726 & 664 & 649 & 1232 & 1129 & 1144 & 865 & 890 & 897 & 899 & 799 & 900 \\
\hline
\end{tabular}

TABLE S82. Wall times of the NMR shielding calculations in seconds (gridsize 2a and seminumerical exchange for the left-hand side of the CPKS equations in the HF and global hybrid calculations).

\begin{tabular}{|c|c|c|c|c|c|c|c|c|c|c|c|c|c|c|c|c|c|c|}
\hline & HF & KT3 & BP86 & PBE & TPSS & BH\&HLYP & B3LYP & PBE0 & TPSSh & LC- $\omega$ PBE & $\omega \mathrm{B} 97 \mathrm{X}-\mathrm{D}$ & CAM-B3LYP & LH07t & LH12ct & LH14t & LH20t & mPSTS & LHJ14 \\
\hline $\mathrm{WO}_{4}^{2-}$ & 12 & 10 & 10 & 10 & 11 & 13 & 13 & 13 & 13 & 19 & 20 & 19 & 25 & 26 & 27 & 22 & 25 & 25 \\
\hline $\mathrm{WO}_{3} \mathrm{~S}^{2-}$ & 63 & 40 & 40 & 47 & 52 & 78 & 62 & 66 & 70 & 97 & 97 & 92 & 216 & 199 & 220 & 220 & 195 & 207 \\
\hline $\mathrm{WO}_{2} \mathrm{~S}_{2}^{2-}$ & 67 & 43 & 40 & 51 & 49 & 84 & 66 & 63 & 58 & 112 & 107 & 100 & 214 & 233 & 224 & 240 & 203 & 216 \\
\hline $\mathrm{WOS}_{3}^{2-}$ & 74 & 53 & 52 & 55 & 54 & 92 & 65 & 66 & 71 & 137 & 149 & 132 & 231 & 250 & 239 & 259 & 223 & 271 \\
\hline $\mathrm{WS}_{4}^{2-}$ & 15 & 13 & 12 & 13 & 12 & 17 & 16 & 16 & 16 & 25 & 20 & 24 & 31 & 34 & 33 & 28 & 30 & 35 \\
\hline $\mathrm{WF}_{6}^{4}$ & 14 & 13 & 13 & 13 & 13 & 16 & 16 & 17 & 13 & 25 & 25 & 19 & 28 & 24 & 29 & 24 & 23 & 28 \\
\hline $\mathrm{WCl}_{6}$ & 17 & 16 & 16 & 16 & 16 & 21 & 16 & 20 & 16 & 31 & 30 & 25 & 38 & 38 & 39 & 32 & 36 & 38 \\
\hline $\mathrm{W}(\mathrm{CO})_{6}$ & 46 & 43 & 42 & 42 & 43 & 56 & 50 & 43 & 50 & 65 & 74 & 63 & 82 & 86 & 87 & 73 & 64 & 79 \\
\hline Total & 308 & 230 & 226 & 247 & 249 & 378 & 304 & 303 & 307 & 511 & 522 & 474 & 865 & 890 & 897 & 899 & 799 & 900 \\
\hline
\end{tabular}


TABLE S83. Wall times of the full SCF procedure in seconds (gridsize 2a, RI- $J$, and seminumerical exchange for the left-hand side of the CPKS equations in the HF and global hybrid calculations).

\begin{tabular}{|c|c|c|c|c|c|c|c|c|c|c|c|c|c|c|c|c|c|c|}
\hline & $\mathrm{HF}$ & KT3 & BP86 & PBE & TPSS & BH\&HLYP & B3LYP & PBE0 & TPSSh & LC- $\omega$ PBE & $\omega \mathrm{B} 97 \mathrm{X}-\mathrm{D}$ & CAM-B3LYP & LH07t & LH12ct & LH14t & LH20t & mPSTS & LHJ14 \\
\hline $\mathrm{WO}_{4}^{2-}$ & 64 & 10 & 4 & 5 & 5 & 53 & 62 & 63 & 61 & 65 & 62 & 57 & 43 & 36 & 33 & 24 & 42 & 34 \\
\hline $\mathrm{WO}_{3} \mathrm{~S}^{2-}$ & 335 & 81 & 22 & 22 & 32 & 356 & 266 & 284 & 268 & 326 & 373 & 353 & 436 & 387 & 415 & 406 & 410 & 384 \\
\hline $\mathrm{WO}_{2} \mathrm{~S}_{2}^{2-}$ & 343 & 115 & 28 & 28 & 39 & 366 & 309 & 316 & 314 & 450 & 456 & 432 & 576 & 536 & 462 & 529 & 512 & 540 \\
\hline $\mathrm{WOS}_{3}^{2-}$ & 374 & 98 & 28 & 27 & 36 & 409 & 365 & 357 & 349 & 473 & 401 & 450 & 506 & 568 & 527 & 477 & 497 & 622 \\
\hline $\mathrm{WS}_{4}^{2-}$ & 80 & 11 & 5 & 5 & 7 & 98 & 83 & 79 & 83 & 100 & 88 & 95 & 42 & 41 & 32 & 45 & 41 & 47 \\
\hline $\mathrm{WF}_{6}$ & 46 & 5 & 2 & 2 & 3 & 50 & 46 & 53 & 46 & 65 & 58 & 57 & 30 & 27 & 25 & 25 & 25 & 29 \\
\hline $\mathrm{WCl}_{6}$ & 85 & 7 & 3 & 3 & 3 & 70 & 57 & 63 & 76 & 89 & 80 & 78 & 36 & 39 & 37 & 36 & 36 & 44 \\
\hline $\mathrm{W}(\mathrm{CO})_{6}$ & 143 & 10 & 5 & 6 & 6 & 145 & 145 & 5 & 128 & 173 & 170 & 173 & 90 & 80 & 83 & 84 & 103 & 70 \\
\hline Total & 1470 & 337 & 97 & 97 & 131 & 1547 & 1333 & 1219 & 1325 & 1740 & 1686 & 1694 & 1758 & 1713 & 1614 & 1626 & 1665 & 1770 \\
\hline
\end{tabular}

TABLE S84. Wall times of the NMR shielding calculations in seconds (gridsize 2a RI- $J$, and seminumerical exchange for the left-hand side of the CPKS equations in the HF and global hybrid calculations).

\begin{tabular}{|c|c|c|c|c|c|c|c|c|c|c|c|c|c|c|c|c|c|c|}
\hline & $\mathrm{HF}$ & KT3 & BP86 & PBE & TPSS & BH\&HLYP & B3LYP & PBE0 & TPSSh & LC- $\omega$ PBE & $\omega \mathrm{B} 97 \mathrm{X}-\mathrm{D}$ & CAM-B3LYP & LH07t & LH12ct & LH14t & LH20t & mPSTS & LHJ14 \\
\hline $\mathrm{WO}_{4}^{2-}$ & 16 & 7 & 8 & 7 & 7 & 15 & 13 & 14 & 14 & 15 & 17 & 14 & 22 & 20 & 21 & 21 & 20 & 23 \\
\hline $\mathrm{WO}_{3} \mathrm{~S}^{2-}$ & 68 & 15 & 15 & 17 & 20 & 78 & 55 & 60 & 57 & 83 & 71 & 72 & 189 & 181 & 188 & 202 & 182 & 179 \\
\hline $\mathrm{WO}_{2} \mathrm{~S}_{2}^{2-}$ & 69 & 16 & 16 & 17 & 20 & 84 & 58 & 60 & 59 & 85 & 77 & 74 & 206 & 265 & 203 & 212 & 182 & 190 \\
\hline $\mathrm{WOS}_{3}^{2-}$ & 78 & 16 & 17 & 16 & 21 & 91 & 73 & 64 & 66 & 91 & 83 & 90 & 203 & 218 & 213 & 231 & 196 & 203 \\
\hline $\mathrm{WS}_{4}^{2-}$ & 16 & 8 & 8 & 8 & 8 & 19 & 16 & 15 & 16 & 19 & 19 & 18 & 26 & 26 & 25 & 26 & 22 & 29 \\
\hline $\mathrm{WF}_{6}^{4}$ & 17 & 10 & 11 & 10 & 11 & 20 & 17 & 18 & 17 & 21 & 19 & 19 & 23 & 22 & 22 & 23 & 24 & 24 \\
\hline $\mathrm{WCl}_{6}$ & 23 & 12 & 12 & 12 & 10 & 24 & 20 & 23 & 19 & 25 & 23 & 24 & 27 & 28 & 29 & 30 & 28 & 33 \\
\hline $\mathrm{W}(\mathrm{CO})_{6}$ & 55 & 32 & 29 & 24 & 32 & 65 & 51 & 30 & 47 & 57 & 58 & 57 & 63 & 63 & 61 & 64 & 63 & 60 \\
\hline Total & 341 & 117 & 116 & 112 & 129 & 396 & 302 & 284 & 295 & 394 & 367 & 367 & 758 & 824 & 762 & 810 & 717 & 740 \\
\hline
\end{tabular}


3. Optimized Structures at the HF Level

The Cartesian coordinates of the structures are listed in Ångström.

\begin{tabular}{|c|c|c|c|}
\hline W & -0.0000000 & 0.0000000 & 0.0000000 \\
\hline 0 & -1.0156131 & 1.0156131 & 1.0156131 \\
\hline 0 & 1.0156131 & -1.0156131 & 1.0156131 \\
\hline 0 & 1.0156131 & 1.0156131 & -1.0156131 \\
\hline 0 & -1.0156131 & -1.0156131 & -1.0156131 \\
\hline W & -0.0007027 & 0.0015073 & -0.0998021 \\
\hline$S$ & 0.0155735 & -0.0324065 & 2.1762058 \\
\hline 0 & 1.6300646 & 0.0044355 & -0.7040141 \\
\hline 0 & -0.8276894 & -1.4024831 & -0.7072271 \\
\hline 0 & -0.8172461 & 1.4289468 & -0.6651626 \\
\hline $\mathrm{S}$ & -0.0113802 & 0.0230339 & 2.2962592 \\
\hline W & 0.0160457 & -0.0199532 & 0.0527368 \\
\hline $\mathrm{S}$ & -1.0094476 & 1.7766347 & -0.8170754 \\
\hline 0 & 1.6469892 & -0.0629283 & -0.4942696 \\
\hline 0 & -0.7740391 & -1.4461731 & -0.4972420 \\
\hline $\mathrm{S}$ & 0.0297055 & 0.0176675 & 2.287018 \\
\hline W & -0.0304055 & -0.0186869 & 0.0719063 \\
\hline $\mathrm{S}$ & -0.9870507 & -1.8826707 & -0.650238 \\
\hline $\mathrm{S}$ & -1.1018228 & 1.7579656 & -0.706681 \\
\hline 0 & 1.5720775 & 0.0228464 & -0.5097081 \\
\hline W & 0.0000000 & -0.0000000 & 0.000000 \\
\hline $\mathrm{S}$ & -1.2653265 & 1.2653265 & 1.265326 \\
\hline $\mathrm{S}$ & 1.2653265 & -1.2653265 & 1.265326 \\
\hline $\mathrm{S}$ & 1.2653265 & 1.2653265 & -1.265326 \\
\hline S & -1.2653265 & -1.2653265 & -1.265326 \\
\hline $\mathrm{F}$ & & 0.0000000 & 0.000000 \\
\hline W & -0.0000000 & -0.0000000 & 0.000000 \\
\hline $\mathrm{F}$ & 0.0000000 & -0.0000000 & -1.811042 \\
\hline $\mathrm{F}$ & -1.8110428 & -0.0000000 & -0.000000 \\
\hline $\mathrm{F}$ & -0.0000000 & 1.8110428 & 0.000000 \\
\hline $\mathrm{F}$ & 0.0000000 & -0.0000000 & 1.811042 \\
\hline $\mathrm{F}$ & 0.0000000 & -1.8110428 & -0.000000 \\
\hline W & 0.0000000 & 0.0000000 & -0.000000 \\
\hline $\mathrm{Cl}$ & -0.0000000 & 2.2873751 & -0.000000 \\
\hline $\mathrm{Cl}$ & 0.0000000 & 0.0000000 & 2.287375 \\
\hline $\mathrm{Cl}$ & -2.2873751 & -0.0000000 & 0.000000 \\
\hline $\mathrm{Cl}$ & 0.0000000 & 0.0000000 & -2.287375 \\
\hline $\mathrm{Cl}$ & & -2.2873751 & 0.000000 \\
\hline $\mathrm{Cl}$ & 2.2873751 & 0.0000000 & 0.000000 \\
\hline
\end{tabular}




$\begin{array}{rrrr}\text { C } & 0.0000000 & 2.1008125 & 0.0000000 \\ \text { C } & 0.0000000 & 3.2127862 & -0.0000000 \\ \text { W } & 0.0000000 & 0.0000000 & 0.0000000 \\ \text { C } & -0.0000000 & -2.1008125 & 0.0000000 \\ 0 & -0.0000000 & -3.2127862 & -0.0000000 \\ \text { C } & -0.0000000 & -0.0000000 & 2.1008125 \\ 0 & -0.0000000 & -0.0000000 & 3.2127862 \\ \text { C } & 2.1008125 & -0.0000000 & -0.0000000 \\ 0 & 3.2127862 & -0.0000000 & -0.0000000 \\ \text { C } & -0.0000000 & -0.0000000 & -2.1008125 \\ 0 & -0.0000000 & -0.0000000 & -3.2127862 \\ \text { C } & -2.1008125 & 0.0000000 & -0.0000000 \\ 0 & -3.2127862 & 0.0000000 & -0.0000000\end{array}$




\section{Optimized Structures at the KT3 Level}

The Cartesian coordinates of the structures are listed in Ångström.

\begin{tabular}{|c|c|c|c|}
\hline W & -0.0000000 & 0.0000000 & -0.0000000 \\
\hline 0 & -1.0295734 & 1.0295734 & 1.0295734 \\
\hline 0 & 1.0295734 & -1.0295734 & 1.0295734 \\
\hline 0 & 1.0295734 & 1.0295734 & -1.0295734 \\
\hline 0 & -1.0295734 & -1.0295734 & -1.0295734 \\
\hline W & -0.0005615 & 0.0012970 & -0.0850081 \\
\hline $\mathrm{S}$ & 0.0155686 & -0.0321690 & 2.1603756 \\
\hline 0 & 1.6570627 & 0.0043115 & -0.7037305 \\
\hline 0 & -0.8413282 & -1.4255892 & -0.7071928 \\
\hline 0 & -0.8307417 & 1.4521498 & -0.6644442 \\
\hline S & -0.0183780 & 0.0362134 & 2.2815096 \\
\hline W & 0.0114777 & -0.0121978 & 0.0585441 \\
\hline S & -1.0060522 & 1.7701554 & -0.7974507 \\
\hline 0 & 1.6769108 & -0.0555540 & -0.4991095 \\
\hline 0 & -0.7957903 & -1.4680029 & -0.5030845 \\
\hline S & 0.0153226 & 0.0167521 & 2.2784150 \\
\hline W & -0.0347085 & -0.0188822 & 0.0734755 \\
\hline$S$ & -0.9955555 & -1.8716938 & -0.6404321 \\
\hline$S$ & -1.1089972 & 1.7466322 & -0.6970575 \\
\hline 0 & 1.6064426 & 0.0243138 & -0.5221050 \\
\hline W & 0.0000000 & -0.0000000 & 0.0000000 \\
\hline$S$ & -1.2647230 & 1.2647230 & 1.2647230 \\
\hline S & 1.2647230 & -1.2647230 & 1.2647230 \\
\hline S & 1.2647230 & 1.2647230 & -1.2647230 \\
\hline S & -1.2647230 & -1.2647230 & -1.2647230 \\
\hline$F$ & 1.8386826 & 0.0000000 & -0.0000000 \\
\hline W & -0.0000000 & -0.0000000 & 0.0000000 \\
\hline $\mathrm{F}$ & 0.0000000 & 0.0000000 & -1.8386826 \\
\hline$F$ & -1.8386826 & -0.0000000 & 0.0000000 \\
\hline $\mathrm{F}$ & -0.0000000 & 1.8386826 & 0.0000000 \\
\hline$F$ & 0.0000000 & 0.0000000 & 1.8386826 \\
\hline $\mathrm{F}$ & 0.0000000 & -1.8386826 & 0.0000000 \\
\hline W & 0.0000000 & 0.0000000 & 0.0000000 \\
\hline $\mathrm{Cl}$ & -0.0000000 & 2.2815417 & -0.0000000 \\
\hline $\mathrm{Cl}$ & 0.0000000 & 0.0000000 & 2.2815417 \\
\hline $\mathrm{Cl}$ & -2.2815417 & -0.0000000 & 0.0000000 \\
\hline $\mathrm{Cl}$ & 0.0000000 & 0.0000000 & -2.2815417 \\
\hline $\mathrm{Cl}$ & 0.0000000 & -2.2815417 & 0.0000000 \\
\hline $\mathrm{Cl}$ & 2.2815417 & 0.0000000 & -0.0000000 \\
\hline
\end{tabular}




$\begin{array}{rrrr}\text { C } & -0.0000000 & 2.0372018 & 0.0000000 \\ \text { O } & -0.0000000 & 3.1854435 & 0.0000000 \\ \text { W } & -0.0000000 & -0.0000000 & -0.0000000 \\ \text { C } & 0.0000000 & -2.0372018 & 0.0000000 \\ 0 & 0.0000000 & -3.1854435 & -0.0000000 \\ \text { C } & -0.0000000 & -0.0000000 & 2.0372018 \\ 0 & 0.0000000 & 0.0000000 & 3.1854435 \\ \text { C } & 2.0372018 & 0.0000000 & -0.0000000 \\ 0 & 3.1854435 & 0.0000000 & -0.0000000 \\ \text { C } & -0.0000000 & -0.0000000 & -2.0372018 \\ 0 & 0.0000000 & 0.0000000 & -3.1854435 \\ \text { C } & -2.0372018 & -0.0000000 & -0.0000000 \\ \text { O } & -3.1854435 & -0.0000000 & 0.0000000\end{array}$


5. Optimized Structures at the BP86 Level

The Cartesian coordinates of the structures are listed in Ångström.

\begin{tabular}{|c|c|c|c|}
\hline W & 0.0000000 & -0.0000000 & 0.0000000 \\
\hline 0 & -1.0387496 & 1.0387496 & 1.0387496 \\
\hline 0 & 1.0387496 & -1.0387496 & 1.0387496 \\
\hline 0 & 1.0387496 & 1.0387496 & -1.0387496 \\
\hline 0 & -1.0387496 & -1.0387496 & -1.0387496 \\
\hline W & -0.0005810 & 0.0013044 & -0.0860213 \\
\hline $\mathrm{S}$ & 0.0156862 & -0.0324538 & 2.1796268 \\
\hline 0 & 1.6721913 & 0.0043589 & -0.7099459 \\
\hline 0 & -0.8489979 & -1.4386589 & -0.7133999 \\
\hline 0 & -0.8382987 & 1.4654494 & -0.6702597 \\
\hline S & -0.0185962 & 0.0363540 & 2.3015648 \\
\hline W & 0.0120139 & -0.0132278 & 0.0577991 \\
\hline S & -1.0150115 & 1.7862166 & -0.8054329 \\
\hline 0 & 1.6928690 & -0.0563305 & -0.5048818 \\
\hline 0 & -0.8031072 & -1.4823982 & -0.5086402 \\
\hline $\mathrm{S}$ & 0.0164983 & 0.0173508 & 2.2994064 \\
\hline W & -0.0337143 & -0.0188610 & 0.0731342 \\
\hline $\mathrm{S}$ & -1.0041191 & -1.8894283 & -0.6477637 \\
\hline $\mathrm{S}$ & -1.1188938 & 1.7635789 & -0.704567 \\
\hline 0 & 1.6227330 & 0.0244816 & -0.5279137 \\
\hline W & 0.0000000 & -0.0000000 & -0.0000000 \\
\hline $\mathrm{S}$ & -1.2772762 & 1.2772762 & 1.2772762 \\
\hline $\mathrm{S}$ & 1.2772762 & -1.2772762 & 1.2772762 \\
\hline S & 1.2772762 & 1.2772762 & -1.2772762 \\
\hline S & -1.2772762 & -1.2772762 & -1.2772762 \\
\hline $\mathrm{F}$ & 1.8559737 & 0.0000000 & 0.0000000 \\
\hline W & -0.0000000 & 0.0000000 & 0.0000000 \\
\hline $\mathrm{F}$ & 0.0000000 & -0.0000000 & -1.8559737 \\
\hline $\mathrm{F}$ & -1.8559737 & -0.0000000 & 0.0000000 \\
\hline $\mathrm{F}$ & -0.0000000 & 1.8559737 & 0.000000 \\
\hline $\mathrm{F}$ & 0.0000000 & -0.0000000 & 1.855973 \\
\hline $\mathrm{F}$ & 0.0000000 & -1.8559737 & 0.000000 \\
\hline W & 0.0000000 & -0.0000000 & 0.0000000 \\
\hline $\mathrm{Cl}$ & -0.0000000 & 2.3076640 & 0.000000 \\
\hline $\mathrm{Cl}$ & 0.0000000 & 0.0000000 & 2.307664 \\
\hline $\mathrm{Cl}$ & -2.3076640 & -0.0000000 & 0.000000 \\
\hline $\mathrm{Cl}$ & -0.0000000 & 0.0000000 & -2.307664 \\
\hline $\mathrm{Cl}$ & 0.0000000 & -2.3076640 & 0.000000 \\
\hline $\mathrm{Cl}$ & 2.3076640 & 0.0000000 & 0.000000 \\
\hline
\end{tabular}




$\begin{array}{rrrr}\text { C } & -0.0000000 & 2.0567229 & 0.0000000 \\ \text { C } & -0.0000000 & 3.2098189 & 0.0000000 \\ \text { W } & 0.0000000 & -0.0000000 & -0.0000000 \\ \text { C } & 0.0000000 & -2.0567229 & 0.0000000 \\ 0 & 0.0000000 & -3.2098189 & 0.0000000 \\ \text { C } & -0.0000000 & 0.0000000 & 2.0567229 \\ 0 & -0.0000000 & 0.0000000 & 3.2098189 \\ \text { C } & 2.0567229 & 0.0000000 & -0.0000000 \\ 0 & 3.2098189 & 0.0000000 & 0.0000000 \\ \text { C } & 0.0000000 & 0.0000000 & -2.0567229 \\ 0 & -0.0000000 & 0.0000000 & -3.2098189 \\ \text { C } & -2.0567229 & -0.0000000 & 0.0000000 \\ 0 & -3.2098189 & -0.0000000 & 0.0000000\end{array}$


6. Optimized Structures at the PBE Level

The Cartesian coordinates of the structures are listed in Ångström.

\begin{tabular}{|c|c|c|c|}
\hline W & -0.0000000 & 0.0000000 & 0.0000000 \\
\hline 0 & -1.0384100 & 1.0384100 & 1.0384100 \\
\hline 0 & 1.0384100 & -1.0384100 & 1.0384100 \\
\hline 0 & 1.0384100 & 1.0384100 & -1.0384100 \\
\hline 0 & -1.0384100 & -1.0384100 & -1.0384100 \\
\hline W & -0.0005800 & 0.0013031 & -0.0858226 \\
\hline $\mathrm{S}$ & 0.0156781 & -0.0324487 & 2.1789531 \\
\hline 0 & 1.6712975 & 0.0043617 & -0.7097849 \\
\hline 0 & -0.8485453 & -1.4378916 & -0.7132268 \\
\hline 0 & -0.8378503 & 1.4646755 & -0.6701188 \\
\hline S & -0.0186358 & 0.0364306 & 2.3004630 \\
\hline W & 0.0119591 & -0.0131309 & 0.0578690 \\
\hline S & -1.0145367 & 1.7853676 & -0.8049034 \\
\hline 0 & 1.6918082 & -0.0564990 & -0.5046285 \\
\hline 0 & -0.8024267 & -1.4815542 & -0.5083912 \\
\hline $\mathrm{S}$ & 0.0162857 & 0.0173324 & 2.2979360 \\
\hline W & -0.0336582 & -0.0188591 & 0.0730997 \\
\hline $\mathrm{S}$ & -1.0036018 & -1.8881781 & -0.6472050 \\
\hline S & -1.1182872 & 1.7623672 & -0.7039515 \\
\hline 0 & 1.6217656 & 0.0244595 & -0.5275831 \\
\hline W & 0.0000000 & -0.0000000 & 0.0000000 \\
\hline $\mathrm{S}$ & -1.2763408 & 1.2763408 & 1.2763408 \\
\hline $\mathrm{S}$ & 1.2763408 & -1.2763408 & 1.2763408 \\
\hline$S$ & 1.2763408 & 1.2763408 & -1.2763408 \\
\hline S & -1.2763408 & -1.2763408 & -1.2763408 \\
\hline $\mathrm{F}$ & 1.8562880 & & 0.0000000 \\
\hline W & -0.0000000 & -0.0000000 & 0.000000 \\
\hline $\mathrm{F}$ & 0.0000000 & 0.0000000 & -1.8562880 \\
\hline $\mathrm{F}$ & -1.8562880 & -0.0000000 & -0.0000000 \\
\hline $\mathrm{F}$ & -0.0000000 & 1.8562880 & 0.000000 \\
\hline $\mathrm{F}$ & 0.0000000 & 0.0000000 & 1.856288 \\
\hline $\mathrm{F}$ & 0.0000000 & -1.8562880 & 0.000000 \\
\hline W & -0.0000000 & -0.0000000 & 0.0000000 \\
\hline $\mathrm{Cl}$ & -0.0000000 & 2.3048336 & 0.0000000 \\
\hline $\mathrm{Cl}$ & 0.0000000 & 0.0000000 & 2.304833 \\
\hline $\mathrm{Cl}$ & -2.3048336 & -0.0000000 & -0.000000 \\
\hline $\mathrm{Cl}$ & 0.0000000 & 0.0000000 & -2.304833 \\
\hline $\mathrm{Cl}$ & 0.0000000 & -2.3048336 & 0.000000 \\
\hline $\mathrm{Cl}$ & 2.3048336 & 0.0000000 & 0.000000 \\
\hline
\end{tabular}




$\begin{array}{rrrr}\text { C } & -0.0000000 & 2.0544066 & -0.0000000 \\ 0 & -0.0000000 & 3.2075894 & 0.0000000 \\ \text { W } & -0.0000000 & -0.0000000 & 0.0000000 \\ \text { C } & 0.0000000 & -2.0544066 & 0.0000000 \\ 0 & 0.0000000 & -3.2075894 & -0.0000000 \\ \text { C } & -0.0000000 & 0.0000000 & 2.0544066 \\ 0 & 0.0000000 & 0.0000000 & 3.2075894 \\ C & 2.0544066 & 0.0000000 & 0.0000000 \\ 0 & 3.2075894 & 0.0000000 & -0.0000000 \\ C & -0.0000000 & 0.0000000 & -2.0544066 \\ 0 & 0.0000000 & 0.0000000 & -3.2075894 \\ C & -2.0544066 & -0.0000000 & 0.0000000 \\ 0 & -3.2075894 & -0.0000000 & 0.0000000\end{array}$




\section{Optimized Structures at the TPSS Level}

The Cartesian coordinates of the structures are listed in Ångström.

\begin{tabular}{|c|c|c|c|}
\hline W & -0.0000000 & 0.0000000 & 0.0000000 \\
\hline 0 & -1.0374341 & 1.0374341 & 1.0374341 \\
\hline 0 & 1.0374341 & -1.0374341 & 1.0374341 \\
\hline 0 & 1.0374341 & 1.0374341 & -1.0374341 \\
\hline 0 & -1.0374341 & -1.0374341 & -1.0374341 \\
\hline W & -0.0005780 & 0.0012927 & -0.0856764 \\
\hline $\mathrm{S}$ & 0.0156473 & -0.0323993 & 2.1771650 \\
\hline 0 & 1.6694484 & 0.0043547 & -0.7092446 \\
\hline 0 & -0.8475997 & -1.4363238 & -0.7126733 \\
\hline 0 & -0.8369180 & 1.4630758 & -0.6695707 \\
\hline S & -0.0183716 & 0.0359112 & 2.2983613 \\
\hline W & 0.0117231 & -0.0127491 & 0.0581661 \\
\hline S & -1.0134048 & 1.7835004 & -0.8045752 \\
\hline 0 & 1.6896146 & -0.0563681 & -0.5039310 \\
\hline 0 & -0.8013933 & -1.4796805 & -0.5076121 \\
\hline S & 0.0163943 & 0.0173679 & 2.2953190 \\
\hline W & -0.0341994 & -0.0188600 & 0.0732803 \\
\hline$S$ & -1.0021775 & -1.8860737 & -0.6464861 \\
\hline$S$ & -1.1168488 & 1.7604043 & -0.7030547 \\
\hline 0 & 1.6193353 & 0.0242835 & -0.5267625 \\
\hline W & -0.0000000 & 0.0000000 & 0.0000000 \\
\hline S & -1.2745880 & 1.2745880 & 1.2745880 \\
\hline S & 1.2745880 & -1.2745880 & 1.2745880 \\
\hline S & 1.2745880 & 1.2745880 & -1.2745880 \\
\hline S & -1.2745880 & -1.2745880 & -1.2745880 \\
\hline$F$ & 1.8514670 & 0.0000000 & 0.0000000 \\
\hline W & -0.0000000 & -0.0000000 & -0.0000000 \\
\hline $\mathrm{F}$ & 0.0000000 & -0.0000000 & -1.8514670 \\
\hline$F$ & -1.8514670 & -0.0000000 & 0.0000000 \\
\hline $\mathrm{F}$ & -0.0000000 & 1.8514670 & 0.0000000 \\
\hline$F$ & 0.0000000 & -0.0000000 & 1.8514670 \\
\hline $\mathrm{F}$ & 0.0000000 & -1.8514670 & -0.0000000 \\
\hline W & -0.0000000 & -0.0000000 & 0.0000000 \\
\hline $\mathrm{Cl}$ & -0.0000000 & 2.3020767 & -0.0000000 \\
\hline $\mathrm{Cl}$ & 0.0000000 & 0.0000000 & 2.3020767 \\
\hline $\mathrm{Cl}$ & -2.3020767 & -0.0000000 & 0.0000000 \\
\hline $\mathrm{Cl}$ & 0.0000000 & 0.0000000 & -2.3020767 \\
\hline $\mathrm{Cl}$ & 0.0000000 & -2.3020767 & 0.0000000 \\
\hline $\mathrm{Cl}$ & 2.3020767 & 0.0000000 & -0.0000000 \\
\hline
\end{tabular}




$\begin{array}{rrrr}\text { C } & -0.0000000 & 2.0596054 & -0.0000000 \\ 0 & -0.0000000 & 3.2105364 & 0.0000000 \\ \text { W } & -0.0000000 & -0.0000000 & 0.0000000 \\ \text { C } & 0.0000000 & -2.0596054 & 0.0000000 \\ 0 & 0.0000000 & -3.2105364 & -0.0000000 \\ \text { C } & -0.0000000 & 0.0000000 & 2.0596054 \\ 0 & 0.0000000 & 0.0000000 & 3.2105364 \\ C & 2.0596054 & 0.0000000 & 0.0000000 \\ 0 & 3.2105364 & 0.0000000 & -0.0000000 \\ C & -0.0000000 & 0.0000000 & -2.0596054 \\ 0 & 0.0000000 & 0.0000000 & -3.2105364 \\ C & -2.0596054 & -0.0000000 & 0.0000000 \\ 0 & -3.2105364 & -0.0000000 & 0.0000000\end{array}$


8. Optimized Structures at the BH\&HLYP Level

The Cartesian coordinates of the structures are listed in Ångström.

\begin{tabular}{|c|c|c|c|}
\hline W & -0.0000000 & 0.0000000 & 0.0000000 \\
\hline 0 & -1.0220443 & 1.0220443 & 1.0220443 \\
\hline 0 & 1.0220443 & -1.0220443 & 1.0220443 \\
\hline 0 & 1.0220443 & 1.0220443 & -1.0220443 \\
\hline 0 & -1.0220443 & -1.0220443 & -1.0220443 \\
\hline W & -0.0006314 & 0.0013942 & -0.0920229 \\
\hline S & 0.0155241 & -0.0322051 & 2.1632182 \\
\hline 0 & 1.6441747 & 0.0043503 & -0.7023258 \\
\hline 0 & -0.8347964 & -1.4146025 & -0.7056512 \\
\hline 0 & -0.8242710 & 1.4410631 & -0.6632183 \\
\hline $\mathrm{S}$ & -0.0159077 & 0.0312203 & 2.2849844 \\
\hline W & 0.0139001 & -0.0162927 & 0.055483 \\
\hline $\mathrm{S}$ & -1.0062842 & 1.7709888 & -0.803943 \\
\hline 0 & 1.6637589 & -0.0574668 & -0.496418 \\
\hline 0 & -0.7872990 & -1.4578356 & $-0.499696 s$ \\
\hline S & 0.0206995 & 0.0172612 & 2.2808497 \\
\hline W & -0.0320298 & -0.0187647 & 0.072495 \\
\hline $\mathrm{S}$ & -0.9919498 & -1.8750945 & -0.643998 \\
\hline $\mathrm{S}$ & -1.1060646 & 1.7502339 & -0.7002602 \\
\hline 0 & 1.5918486 & 0.0234861 & -0.516790 \\
\hline W & -0.0000000 & 0.0000000 & 0.0000000 \\
\hline $\mathrm{S}$ & -1.2648467 & 1.2648467 & 1.2648467 \\
\hline $\mathrm{S}$ & 1.2648467 & -1.2648467 & 1.264846 \\
\hline S & 1.2648467 & 1.2648467 & -1.264846 \\
\hline$S$ & -1.2648467 & -1.2648467 & -1.264846 \\
\hline $\mathrm{F}$ & 1.8226982 & 0.0000000 & 0.0000000 \\
\hline W & -0.0000000 & -0.0000000 & 0.000000 \\
\hline$F$ & 0.0000000 & 0.0000000 & -1.822698 \\
\hline $\mathrm{F}$ & -1.8226982 & -0.0000000 & 0.000000 \\
\hline $\mathrm{F}$ & -0.0000000 & 1.8226982 & 0.000000 \\
\hline $\mathrm{F}$ & 0.0000000 & 0.0000000 & 1.822698 \\
\hline $\mathrm{F}$ & 0.0000000 & -1.8226982 & -0.000000 \\
\hline W & -0.0000000 & -0.0000000 & -0.000000 \\
\hline $\mathrm{Cl}$ & -0.0000000 & 2.2845391 & -0.000000 \\
\hline $\mathrm{Cl}$ & -0.0000000 & -0.0000000 & 2.284539 \\
\hline $\mathrm{Cl}$ & -2.2845391 & -0.0000000 & 0.000000 \\
\hline $\mathrm{Cl}$ & -0.0000000 & -0.0000000 & -2.2845391 \\
\hline $\mathrm{Cl}$ & 0.0000000 & -2.2845391 & 0.0000000 \\
\hline $\mathrm{Cl}$ & 2.2845391 & 0.0000000 & -0.000000 \\
\hline
\end{tabular}




$\begin{array}{rrrr}\text { C } & -0.0000000 & 2.0663875 & 0.0000000 \\ \text { C } & -0.0000000 & 3.1910633 & 0.0000000 \\ \text { W } & 0.0000000 & 0.0000000 & 0.0000000 \\ \text { C } & 0.0000000 & -2.0663875 & 0.0000000 \\ 0 & 0.0000000 & -3.1910633 & 0.0000000 \\ \text { C } & -0.0000000 & -0.0000000 & 2.0663875 \\ 0 & 0.0000000 & -0.0000000 & 3.1910633 \\ \text { C } & 2.0663875 & 0.0000000 & 0.0000000 \\ 0 & 3.1910633 & 0.0000000 & -0.0000000 \\ \text { C } & -0.0000000 & -0.0000000 & -2.0663875 \\ 0 & 0.0000000 & -0.0000000 & -3.1910633 \\ \text { C } & -2.0663875 & -0.0000000 & 0.0000000 \\ 0 & -3.1910633 & -0.0000000 & 0.0000000\end{array}$


9. Optimized Structures at the B3LYP Level

The Cartesian coordinates of the structures are listed in Ångström.

\begin{tabular}{|c|c|c|c|}
\hline W & 0.0000000 & 0.0000000 & 0.0000000 \\
\hline 0 & -1.0336129 & 1.0336129 & 1.0336129 \\
\hline 0 & 1.0336129 & -1.0336129 & 1.0336129 \\
\hline 0 & 1.0336129 & 1.0336129 & -1.0336129 \\
\hline 0 & -1.0336129 & -1.0336129 & -1.0336129 \\
\hline & -0.0006061 & 0.0013530 & -0.089475 \\
\hline ד & 0.0156586 & -0.0323983 & 2.1768353 \\
\hline U & 1.6641167 & 0.0043496 & -0.7078143 \\
\hline 0 & -0.8449053 & -1.4317180 & -0.7112529 \\
\hline 0 & -0.8342639 & 1.4584136 & -0.6682926 \\
\hline 5 & -0.0173289 & 0.0339245 & 2.2996271 \\
\hline W & 0.0131464 & -0.0150991 & 0.0563819 \\
\hline S & -1.0135623 & 1.7836942 & -0.8071376 \\
\hline 0 & 1.6848348 & -0.0564246 & -0.5024565 \\
\hline 0 & -0.7989220 & -1.4754811 & -0.5060059 \\
\hline$S$ & 0.0186897 & 0.0174193 & 2.297465 \\
\hline W & -0.0326414 & -0.0188113 & 0.072742 \\
\hline $\mathrm{S}$ & -1.0013254 & -1.8883907 & -0.648223 \\
\hline $\mathrm{S}$ & -1.1161360 & 1.7627374 & -0.704947 \\
\hline 0 & 1.6139171 & 0.0241673 & -0.5247407 \\
\hline WV & -0.0000000 & 0.0000000 & 0.0000000 \\
\hline $\mathrm{S}$ & -1.2757310 & 1.2757310 & 1.2757310 \\
\hline $\mathrm{S}$ & 1.2757310 & -1.2757310 & 1.2757310 \\
\hline S & 1.2757310 & 1.2757310 & -1.2757310 \\
\hline S & -1.2757310 & -1.2757310 & -1.2757310 \\
\hline $\mathrm{F}$ & 1.8452016 & 0.0000000 & 0.0000000 \\
\hline W & -0.0000000 & -0.0000000 & -0.000000 \\
\hline $\mathrm{F}$ & 0.0000000 & 0.0000000 & -1.8452016 \\
\hline $\mathrm{F}$ & -1.8452016 & -0.0000000 & -0.000000 \\
\hline $\mathrm{F}$ & -0.0000000 & 1.8452016 & -0.000000 \\
\hline $\mathrm{F}$ & 0.0000000 & 0.0000000 & 1.845201 \\
\hline $\mathrm{F}$ & 0.0000000 & -1.8452016 & 0.000000 \\
\hline W & -0.0000000 & -0.0000000 & -0.0000000 \\
\hline $\mathrm{Cl}$ & -0.0000000 & 2.3061749 & 0.000000 \\
\hline $\mathrm{Cl}$ & 0.0000000 & 0.0000000 & 2.306174 \\
\hline $\mathrm{Cl}$ & -2.3061749 & -0.0000000 & 0.000000 \\
\hline $\mathrm{Cl}$ & 0.0000000 & 0.0000000 & -2.306174 \\
\hline $\mathrm{Cl}$ & 0.0000000 & -2.3061749 & 0.000000 \\
\hline $\mathrm{Cl}$ & 2.3061749 & 0.0000000 & -0.000000 \\
\hline
\end{tabular}




$\begin{array}{rrrr}\text { C } & 0.0000000 & 2.0677525 & -0.0000000 \\ \text { O } & 0.0000000 & 3.2080573 & 0.0000000 \\ \text { W } & 0.0000000 & 0.0000000 & 0.0000000 \\ \text { C } & -0.0000000 & -2.0677525 & 0.0000000 \\ 0 & -0.0000000 & -3.2080573 & -0.0000000 \\ \text { C } & -0.0000000 & 0.0000000 & 2.0677525 \\ 0 & 0.0000000 & 0.0000000 & 3.2080573 \\ \text { C } & 2.0677525 & -0.0000000 & -0.0000000 \\ 0 & 3.2080573 & -0.0000000 & 0.0000000 \\ \text { C } & -0.0000000 & 0.0000000 & -2.0677525 \\ 0 & 0.0000000 & 0.0000000 & -3.2080573 \\ \text { C } & -2.0677525 & 0.0000000 & 0.0000000 \\ \text { O } & -3.2080573 & 0.0000000 & 0.0000000\end{array}$


10. Optimized Structures at the PBEO Level

The Cartesian coordinates of the structures are listed in Ångström.

\begin{tabular}{|c|c|c|c|}
\hline W & -0.0000000 & 0.0000000 & 0.0000000 \\
\hline 0 & -1.0263919 & 1.0263919 & 1.0263919 \\
\hline J & 1.0263919 & -1.0263919 & 1.0263919 \\
\hline 0 & 1.0263919 & 1.0263919 & -1.0263919 \\
\hline 0 & -1.0263919 & -1.0263919 & -1.0263919 \\
\hline & -0.0006029 & 0.0013383 & -0.0882223 \\
\hline S & 0.0155497 & -0.0322261 & 2.1643160 \\
\hline 0 & 1.6508462 & 0.0043515 & -0.7039859 \\
\hline 0 & -0.8381803 & -1.4203224 & -0.7073515 \\
\hline 0 & -0.8276128 & 1.4468586 & -0.6647564 \\
\hline S & -0.0174250 & 0.0341143 & 2.284707 \\
\hline W & 0.0127473 & -0.0144009 & 0.056910 \\
\hline S & -1.0068468 & 1.7719475 & -0.8008511 \\
\hline 0 & 1.6705727 & -0.0573581 & -0.498427 \\
\hline 0 & -0.7908802 & -1.4636888 & -0.5019308 \\
\hline $\mathrm{S}$ & 0.0178081 & 0.0171368 & 2.280478 \\
\hline W & -0.0327828 & -0.0188090 & 0.072783 \\
\hline $\mathrm{S}$ & -0.9942523 & -1.8740411 & -0.642483 \\
\hline $\mathrm{S}$ & -1.1081891 & 1.7490450 & -0.6987565 \\
\hline 0 & 1.5999201 & 0.0237903 & -0.5197251 \\
\hline W & -0.0000000 & 0.0000000 & 0.000000 \\
\hline $\mathrm{S}$ & -1.2651581 & 1.2651581 & 1.265158 \\
\hline $\mathrm{S}$ & 1.2651581 & -1.2651581 & 1.265158 \\
\hline S & 1.2651581 & 1.2651581 & -1.265158 \\
\hline S & -1.2651581 & -1.2651581 & -1.265158 \\
\hline $\mathrm{F}$ & 1.8323025 & 0.0000000 & -0.000000 \\
\hline W & 0.0000000 & -0.0000000 & -0.000000 \\
\hline $\mathrm{F}$ & 0.0000000 & 0.0000000 & -1.832302 \\
\hline $\mathrm{F}$ & -1.8323025 & -0.0000000 & 0.000000 \\
\hline $\mathrm{F}$ & -0.0000000 & 1.8323025 & -0.000000 \\
\hline $\mathrm{F}$ & 0.0000000 & 0.0000000 & 1.832302 \\
\hline $\mathrm{F}$ & 0.0000000 & -1.8323025 & -0.000000 \\
\hline W & 0.0000000 & -0.0000000 & 0.000000 \\
\hline $\mathrm{Cl}$ & -0.0000000 & 2.2825643 & -0.000000 \\
\hline $\mathrm{Cl}$ & 0.0000000 & 0.0000000 & 2.282564 \\
\hline $\mathrm{Cl}$ & -2.2825643 & -0.0000000 & 0.000000 \\
\hline $\mathrm{Cl}$ & 0.0000000 & 0.0000000 & -2.282564 \\
\hline $\mathrm{Cl}$ & & -2.2825643 & -0.000000 \\
\hline $\mathrm{Cl}$ & 2.2825643 & 0.0000000 & -0.000000 \\
\hline
\end{tabular}




$\begin{array}{rrrr}\text { C } & -0.0000000 & 2.0544066 & -0.0000000 \\ \text { O } & -0.0000000 & 3.2075894 & 0.0000000 \\ \text { W } & -0.0000000 & -0.0000000 & 0.0000000 \\ \text { C } & 0.0000000 & -2.0544066 & 0.0000000 \\ 0 & 0.0000000 & -3.2075894 & -0.0000000 \\ \text { C } & -0.0000000 & 0.0000000 & 2.0544066 \\ 0 & 0.0000000 & 0.0000000 & 3.2075894 \\ \text { C } & 2.0544066 & 0.0000000 & 0.0000000 \\ 0 & 3.2075894 & 0.0000000 & -0.0000000 \\ \text { C } & -0.0000000 & 0.0000000 & -2.0544066 \\ 0 & 0.0000000 & 0.0000000 & -3.2075894 \\ \text { C } & -2.0544066 & -0.0000000 & 0.0000000 \\ \text { O } & -3.2075894 & -0.0000000 & 0.0000000\end{array}$


11. Optimized Structures at the TPSSh Level

The Cartesian coordinates of the structures are listed in Ångström.

\begin{tabular}{|c|c|c|c|}
\hline W & 0.0000000 & -0.0000000 & 0.0000000 \\
\hline 0 & -1.0326034 & 1.0326034 & 1.0326034 \\
\hline 0 & 1.0326034 & -1.0326034 & 1.0326034 \\
\hline 0 & 1.0326034 & 1.0326034 & -1.0326034 \\
\hline 0 & -1.0326034 & -1.0326034 & -1.0326034 \\
\hline W & -0.0005866 & 0.0013127 & -0.0866902 \\
\hline $\mathrm{S}$ & 0.0156021 & -0.0323148 & 2.1712498 \\
\hline 0 & 1.6612287 & 0.0043493 & -0.7068788 \\
\hline 0 & -0.8434366 & -1.4292566 & -0.7102839 \\
\hline 0 & -0.8328076 & 1.4559095 & -0.667396 \\
\hline $\mathrm{S}$ & -0.0179058 & 0.0350320 & 2.2920402 \\
\hline W & 0.0120775 & -0.0133141 & 0.0577351 \\
\hline $\mathrm{S}$ & -1.0103430 & 1.7781226 & -0.8028997 \\
\hline 0 & 1.6811291 & -0.0567105 & -0.501434 \\
\hline 0 & -0.7967898 & -1.4725160 & -0.505032 \\
\hline S & 0.0169934 & 0.0172682 & 2.2883806 \\
\hline W & -0.0337872 & -0.0188343 & 0.073129 \\
\hline $\mathrm{S}$ & -0.9984837 & -1.8804359 & -0.644590 \\
\hline S & -1.1128451 & 1.7550924 & -0.701002 \\
\hline 0 & 1.6106265 & 0.0240317 & -0.523620 \\
\hline W & 0.0000000 & -0.0000000 & 0.0000000 \\
\hline $\mathrm{S}$ & -1.2701522 & 1.2701522 & 1.2701522 \\
\hline $\mathrm{S}$ & 1.2701522 & -1.2701522 & 1.270152 \\
\hline S & 1.2701522 & 1.2701522 & -1.270152 \\
\hline$S$ & -1.2701522 & -1.2701522 & -1.270152 \\
\hline $\mathrm{F}$ & 1.8421725 & -0.0000000 & -0.0000000 \\
\hline W & 0.0000000 & 0.0000000 & -0.000000 \\
\hline$F$ & 0.0000000 & -0.0000000 & -1.842172 \\
\hline $\mathrm{F}$ & -1.8421725 & 0.0000000 & -0.00000 \\
\hline $\mathrm{F}$ & 0.0000000 & 1.8421725 & -0.000000 \\
\hline $\mathrm{F}$ & 0.0000000 & -0.0000000 & 1.842172 \\
\hline $\mathrm{F}$ & -0.0000000 & -1.8421725 & -0.000000 \\
\hline W & 0.0000000 & 0.0000000 & 0.000000 \\
\hline $\mathrm{Cl}$ & -0.0000000 & & -0.000000 \\
\hline $\mathrm{Cl}$ & 0.0000000 & -0.0000000 & 2.293172 \\
\hline $\mathrm{Cl}$ & -2.2931727 & -0.0000000 & 0.000000 \\
\hline $\mathrm{Cl}$ & 0.0000000 & 0.0000000 & -2.2931727 \\
\hline $\mathrm{Cl}$ & 0.0000000 & -2.2931727 & 0.0000000 \\
\hline $\mathrm{Cl}$ & 2.2931727 & 0.0000000 & 0.000000 \\
\hline
\end{tabular}




$\begin{array}{rrrr}\text { C } & -0.0000000 & 2.0570167 & -0.0000000 \\ \text { C } & -0.0000000 & 3.2017195 & 0.0000000 \\ \text { W } & -0.0000000 & -0.0000000 & -0.0000000 \\ \text { C } & 0.0000000 & -2.0570167 & 0.0000000 \\ 0 & 0.0000000 & -3.2017195 & 0.0000000 \\ \text { C } & -0.0000000 & 0.0000000 & 2.0570167 \\ 0 & -0.0000000 & 0.0000000 & 3.2017195 \\ \text { C } & 2.0570167 & 0.0000000 & -0.0000000 \\ 0 & 3.2017195 & 0.0000000 & 0.0000000 \\ \text { C } & -0.0000000 & 0.0000000 & -2.0570167 \\ 0 & -0.0000000 & 0.0000000 & -3.2017195 \\ \text { C } & -2.0570167 & -0.0000000 & -0.0000000 \\ 0 & -3.2017195 & -0.0000000 & 0.0000000\end{array}$


12. Optimized Structures at the LC- $\omega P B E$ Level

The Cartesian coordinates of the structures are listed in Ångström.

\begin{tabular}{|c|c|c|c|}
\hline W & -0.0000000 & 0.0000000 & 0.0000000 \\
\hline 0 & -1.0222343 & 1.0222343 & 1.0222343 \\
\hline 0 & 1.0222343 & -1.0222343 & 1.0222343 \\
\hline 0 & 1.0222343 & 1.0222343 & -1.0222343 \\
\hline 0 & -1.0222343 & -1.0222343 & -1.0222343 \\
\hline $\boldsymbol{W}$ & -0.0005931 & 0.0013430 & -0.0880258 \\
\hline S & 0.0154826 & -0.0321173 & 2.1561258 \\
\hline 0 & 1.6419224 & 0.0043427 & -0.7012647 \\
\hline 0 & -0.8336582 & -1.4126167 & -0.7045877 \\
\hline 0 & -0.8231536 & 1.4390483 & -0.662247 \\
\hline $\mathrm{S}$ & -0.0159928 & 0.0316601 & 2.2745753 \\
\hline W & 0.0118311 & -0.0128352 & 0.0580843 \\
\hline $\mathrm{S}$ & -1.0016657 & 1.7629143 & -0.7993053 \\
\hline 0 & 1.6581945 & -0.0578196 & $-0.494724 c$ \\
\hline 0 & -0.7841992 & -1.4533056 & -0.498221 \\
\hline S & 0.0194263 & 0.0169745 & 2.2665495 \\
\hline W & -0.0351989 & -0.0188730 & 0.073662 \\
\hline $\mathrm{S}$ & -0.9865673 & -1.8628723 & -0.6388966 \\
\hline S & -1.0998142 & 1.7384981 & -0.694829 \\
\hline 0 & 1.5846581 & 0.0233946 & -0.514189 \\
\hline W & -0.0000000 & 0.0000000 & 0.0000000 \\
\hline $\mathrm{S}$ & -1.2549996 & 1.2549996 & 1.2549996 \\
\hline $\mathrm{S}$ & 1.2549996 & -1.2549996 & 1.254999 \\
\hline S & 1.2549996 & 1.2549996 & -1.254999 \\
\hline$S$ & -1.2549996 & -1.2549996 & -1.254999 \\
\hline $\mathrm{F}$ & 1.8299081 & -0.0000000 & -0.0000000 \\
\hline W & -0.0000000 & -0.0000000 & -0.000000 \\
\hline$F$ & 0.0000000 & -0.0000000 & -1.829908 \\
\hline $\mathrm{F}$ & -1.8299081 & 0.0000000 & -0.000000 \\
\hline $\mathrm{F}$ & 0.0000000 & 1.8299081 & -0.000000 \\
\hline $\mathrm{F}$ & 0.0000000 & -0.0000000 & 1.829908 \\
\hline $\mathrm{F}$ & -0.0000000 & -1.8299081 & 0.000000 \\
\hline W & -0.0000000 & -0.0000000 & -0.000000 \\
\hline $\mathrm{Cl}$ & 0.0000000 & 2.2664316 & -0.000000 \\
\hline $\mathrm{Cl}$ & 0.0000000 & 0.0000000 & 2.266431 \\
\hline $\mathrm{Cl}$ & -2.2664316 & 0.0000000 & -0.000000 \\
\hline $\mathrm{Cl}$ & 0.0000000 & 0.0000000 & -2.2664316 \\
\hline $\mathrm{Cl}$ & -0.0000000 & -2.2664316 & 0.0000000 \\
\hline $\mathrm{Cl}$ & 2.2664316 & -0.0000000 & -0.000000 \\
\hline
\end{tabular}




$\begin{array}{rrrr}\text { C } & -0.0000000 & 2.0449330 & 0.0000000 \\ \text { O } & -0.0000000 & 3.1789850 & -0.0000000 \\ \text { W } & -0.0000000 & -0.0000000 & 0.0000000 \\ \text { C } & 0.0000000 & -2.0449330 & 0.0000000 \\ 0 & 0.0000000 & -3.1789850 & -0.0000000 \\ \text { C } & -0.0000000 & 0.0000000 & 2.0449330 \\ 0 & 0.0000000 & 0.0000000 & 3.1789850 \\ \text { C } & 2.0449330 & 0.0000000 & -0.0000000 \\ 0 & 3.1789850 & 0.0000000 & -0.0000000 \\ \text { C } & -0.0000000 & 0.0000000 & -2.0449330 \\ 0 & 0.0000000 & 0.0000000 & -3.1789850 \\ \text { C } & -2.0449330 & -0.0000000 & -0.0000000 \\ \text { O } & -3.1789850 & -0.0000000 & -0.0000000\end{array}$


13. Optimized Structures at the $\omega B 97 X-D$ Level

The Cartesian coordinates of the structures are listed in Ångström.

\begin{tabular}{|c|c|c|c|}
\hline W & -0.0000000 & 0.0000000 & 0.0000000 \\
\hline 0 & -1.0266089 & 1.0266089 & 1.0266089 \\
\hline 0 & 1.0266089 & -1.0266089 & 1.0266089 \\
\hline 0 & 1.0266089 & 1.0266089 & -1.0266089 \\
\hline 0 & -1.0266089 & -1.0266089 & -1.0266089 \\
\hline W & -0.0006271 & 0.0013518 & -0.0892349 \\
\hline $\mathrm{S}$ & 0.0156235 & -0.0323404 & 2.1718794 \\
\hline 0 & 1.6490069 & 0.0043909 & -0.7061389 \\
\hline 0 & -0.8372792 & -1.4187281 & -0.7095246 \\
\hline 0 & -0.8267241 & 1.4453259 & -0.6669810 \\
\hline $\mathrm{S}$ & -0.0149426 & 0.0296957 & 2.2919542 \\
\hline W & 0.0126350 & -0.0141665 & 0.057073 \\
\hline $\mathrm{S}$ & -1.0090949 & 1.7758934 & -0.808434 \\
\hline 0 & 1.6671005 & -0.0591939 & -0.498370 \\
\hline 0 & -0.7875300 & -1.4616147 & -0.501813 \\
\hline S & 0.0221237 & 0.0173697 & 2.285807 \\
\hline W & -0.0336782 & -0.0188409 & 0.073109 \\
\hline $\mathrm{S}$ & -0.9931510 & -1.8796065 & -0.6462246 \\
\hline $\mathrm{S}$ & -1.1074189 & 1.7545379 & -0.702650 \\
\hline 0 & 1.5946284 & 0.0236618 & -0.517746 \\
\hline W & -0.0000000 & 0.0000000 & 0.0000000 \\
\hline S & -1.2670234 & 1.2670234 & 1.2670234 \\
\hline $\mathrm{S}$ & 1.2670234 & -1.2670234 & 1.267023 \\
\hline S & 1.2670234 & 1.2670234 & -1.267023 \\
\hline$S$ & -1.2670234 & -1.2670234 & -1.267023 \\
\hline $\mathrm{F}$ & 1.8352661 & -0.0000000 & 0.0000000 \\
\hline W & 0.0000000 & 0.0000000 & 0.0000000 \\
\hline $\mathrm{F}$ & -0.0000000 & 0.0000000 & -1.835266 \\
\hline $\mathrm{F}$ & -1.8352661 & 0.0000000 & 0.000000 \\
\hline $\mathrm{F}$ & 0.0000000 & 1.8352661 & 0.000000 \\
\hline $\mathrm{F}$ & -0.0000000 & 0.0000000 & 1.835266 \\
\hline $\mathrm{F}$ & -0.0000000 & -1.8352661 & 0.000000 \\
\hline W & -0.0000000 & -0.0000000 & 0.000000 \\
\hline $\mathrm{Cl}$ & -0.0000000 & 2.2851836 & -0.000000 \\
\hline $\mathrm{Cl}$ & 0.0000000 & 0.0000000 & 2.285183 \\
\hline $\mathrm{Cl}$ & -2.2851836 & -0.0000000 & -0.000000 \\
\hline $\mathrm{Cl}$ & 0.0000000 & 0.0000000 & -2.285183 \\
\hline $\mathrm{Cl}$ & 0.0000000 & -2.2851836 & -0.0000000 \\
\hline $\mathrm{Cl}$ & 2.2851836 & 0.0000000 & 0.000000 \\
\hline
\end{tabular}




$\begin{array}{rrrr}\text { C } & -0.0000000 & 2.0588607 & 0.0000000 \\ \text { O } & -0.0000000 & 3.1947507 & 0.0000000 \\ \text { W } & 0.0000000 & 0.0000000 & -0.0000000 \\ \text { C } & 0.0000000 & -2.0588607 & -0.0000000 \\ 0 & 0.0000000 & -3.1947507 & 0.0000000 \\ \text { C } & 0.0000000 & 0.0000000 & 2.0588607 \\ 0 & 0.0000000 & 0.0000000 & 3.1947507 \\ \text { C } & 2.0588607 & 0.0000000 & 0.0000000 \\ 0 & 3.1947507 & 0.0000000 & 0.0000000 \\ \text { C } & 0.0000000 & 0.0000000 & -2.0588607 \\ 0 & 0.0000000 & -0.0000000 & -3.1947507 \\ \text { C } & -2.0588607 & -0.0000000 & -0.0000000 \\ \text { D } & -3.1947507 & -0.0000000 & 0.0000000\end{array}$


14. Optimized Structures at the CAM-B3LYP Level

The Cartesian coordinates of the structures are listed in Ångström.

\begin{tabular}{|c|c|c|c|}
\hline W & 0.0000000 & -0.0000000 & -0.0000000 \\
\hline 0 & -1.0267875 & 1.0267875 & 1.0267875 \\
\hline כ & 1.0267875 & -1.0267875 & 1.0267875 \\
\hline 0 & 1.0267875 & 1.0267875 & -1.0267875 \\
\hline 0 & -1.0267875 & -1.0267875 & -1.0267875 \\
\hline W & -0.0006035 & 0.0013548 & -0.089331 \\
\hline $\mathrm{S}$ & 0.0155510 & -0.0322166 & 2.1639230 \\
\hline 0 & 1.6520750 & 0.0043357 & -0.7034877 \\
\hline 0 & -0.8387966 & -1.4213679 & -0.7068676 \\
\hline 0 & -0.8282259 & 1.4478940 & -0.6642365 \\
\hline S & -0.0166429 & 0.0326831 & 2.285500 \\
\hline W & 0.0127233 & -0.0143480 & 0.0569482 \\
\hline S & -1.0068585 & 1.7720120 & -0.8026646 \\
\hline 0 & 1.6710483 & -0.0562010 & -0.4979636 \\
\hline 0 & -0.7921022 & -1.4635322 & -0.5014117 \\
\hline $\mathrm{S}$ & 0.0190011 & 0.0172114 & 2.281380 \\
\hline W & -0.0338019 & -0.0188354 & 0.073142 \\
\hline $\mathrm{S}$ & -0.9936989 & -1.8750930 & -0.6433533 \\
\hline $\mathrm{S}$ & -1.1077208 & 1.7501018 & -0.6996237 \\
\hline 0 & 1.5987245 & 0.0237371 & -0.5192507 \\
\hline W & 0.0000000 & 0.0000000 & 0.0000000 \\
\hline $\mathrm{S}$ & -1.2652465 & 1.2652465 & 1.2652465 \\
\hline $\mathrm{S}$ & 1.2652465 & -1.2652465 & 1.2652465 \\
\hline $\mathrm{S}$ & 1.2652465 & 1.2652465 & -1.2652465 \\
\hline S & -1.2652465 & -1.2652465 & -1.2652465 \\
\hline $\mathrm{F}$ & 1.8334593 & & -0.0000000 \\
\hline W & -0.0000000 & -0.0000000 & 0.000000 \\
\hline $\mathrm{F}$ & 0.0000000 & 0.0000000 & -1.8334593 \\
\hline $\mathrm{F}$ & -1.8334593 & -0.0000000 & 0.000000 \\
\hline $\mathrm{F}$ & -0.0000000 & 1.8334593 & -0.000000 \\
\hline $\mathrm{F}$ & 0.0000000 & 0.00 & 1.833459 \\
\hline $\mathrm{F}$ & 0.0000000 & -1.8334593 & 0.000000 \\
\hline W & -0.0000000 & -0.0000000 & 0.0000000 \\
\hline $\mathrm{Cl}$ & -0.0000000 & 2.2863867 & 0.000000 \\
\hline $\mathrm{Cl}$ & 0.0000000 & -0.0000000 & 2.286386 \\
\hline $\mathrm{Cl}$ & -2.2863867 & -0.0000000 & 0.000000 \\
\hline $\mathrm{Cl}$ & 0.0000000 & -0.0000000 & -2.286386 \\
\hline $\mathrm{Cl}$ & 0.0000000 & -2.2863867 & -0.000000 \\
\hline $\mathrm{Cl}$ & 2.2863867 & 0.0000000 & -0.000000 \\
\hline
\end{tabular}




$\begin{array}{rrrr}\text { C } & -0.0000000 & 2.0591770 & -0.0000000 \\ \text { O } & -0.0000000 & 3.1931929 & -0.0000000 \\ \text { W } & -0.0000000 & -0.0000000 & -0.0000000 \\ \text { C } & 0.0000000 & -2.0591770 & -0.0000000 \\ 0 & 0.0000000 & -3.1931929 & -0.0000000 \\ \text { C } & -0.0000000 & 0.0000000 & 2.0591770 \\ 0 & 0.0000000 & 0.0000000 & 3.1931929 \\ \text { C } & 2.0591770 & 0.0000000 & -0.0000000 \\ 0 & 3.1931929 & 0.0000000 & 0.0000000 \\ \text { C } & -0.0000000 & 0.0000000 & -2.0591770 \\ 0 & 0.0000000 & 0.0000000 & -3.1931929 \\ \text { C } & -2.0591770 & -0.0000000 & -0.0000000 \\ \text { O } & -3.1931929 & -0.0000000 & -0.0000000\end{array}$


15. Optimized Structures at the CAM-QTP-OO Level

The Cartesian coordinates of the structures are listed in Ångström.

\begin{tabular}{|c|c|c|c|}
\hline W & -0.0000000 & 0.0000000 & 0.0000000 \\
\hline 0 & -1.0158681 & 1.0158681 & 1.0158681 \\
\hline 0 & 1.0158681 & -1.0158681 & 1.0158681 \\
\hline 0 & 1.0158681 & 1.0158681 & -1.0158681 \\
\hline 0 & -1.0158681 & -1.0158681 & -1.0158681 \\
\hline W & -0.0006333 & 0.0013915 & -0.0919009 \\
\hline S & 0.0154337 & -0.0320368 & 2.1519626 \\
\hline 0 & 1.6332273 & 0.0043320 & -0.6985471 \\
\hline 0 & -0.8292392 & -1.4051924 & -0.7018336 \\
\hline 0 & -0.8187885 & 1.4315058 & -0.659681 \\
\hline $\mathrm{S}$ & -0.0153840 & 0.0302591 & 2.2728309 \\
\hline W & 0.0135817 & -0.0157393 & 0.0558980 \\
\hline $\mathrm{S}$ & -1.0005256 & 1.7609756 & -0.7999773 \\
\hline 0 & 1.6514286 & -0.0575679 & -0.492583 \\
\hline 0 & -0.7809326 & -1.4473136 & -0.495759 \\
\hline S & 0.0209841 & 0.0170704 & 2.2670970 \\
\hline W & -0.0328059 & -0.0187888 & 0.072795 \\
\hline $\mathrm{S}$ & -0.9854112 & -1.8637217 & -0.6398410 \\
\hline $\mathrm{S}$ & -1.0988568 & 1.7394443 & -0.695739 \\
\hline 0 & 1.5785939 & 0.0231179 & -0.512015 \\
\hline W & -0.0000000 & 0.0000000 & -0.0000000 \\
\hline S & -1.2561030 & 1.2561030 & 1.2561030 \\
\hline $\mathrm{S}$ & 1.2561030 & -1.2561030 & 1.256103 \\
\hline S & 1.2561030 & 1.2561030 & -1.256103 \\
\hline$S$ & -1.2561030 & -1.2561030 & -1.256103 \\
\hline $\mathrm{F}$ & 1.8116457 & 0.0000000 & 0.0000000 \\
\hline W & 0.0000000 & 0.0000000 & -0.000000 \\
\hline$F$ & -0.0000000 & 0.0000000 & -1.811645 \\
\hline $\mathrm{F}$ & -1.8116457 & -0.0000000 & -0.000000 \\
\hline $\mathrm{F}$ & -0.0000000 & 1.8116457 & 0.000000 \\
\hline $\mathrm{F}$ & -0.0000000 & 0.0000000 & 1.811645 \\
\hline $\mathrm{F}$ & 0.0000000 & -1.8116457 & 0.000000 \\
\hline W & -0.0000000 & -0.0000000 & 0.000000 \\
\hline $\mathrm{Cl}$ & 0.0000000 & 2.2679378 & -0.000000 \\
\hline $\mathrm{Cl}$ & 0.0000000 & 0.0000000 & 2.267937 \\
\hline $\mathrm{Cl}$ & -2.2679378 & 0.0000000 & -0.000000 \\
\hline $\mathrm{Cl}$ & 0.0000000 & 0.0000000 & -2.267937 \\
\hline $\mathrm{Cl}$ & -0.0000000 & -2.2679378 & -0.0000000 \\
\hline $\mathrm{Cl}$ & 2.2679378 & -0.0000000 & -0.000000 \\
\hline
\end{tabular}




$\begin{array}{rrrr}\text { C } & -0.0000000 & 2.0580351 & -0.0000000 \\ \text { O } & -0.0000000 & 3.1766053 & 0.0000000 \\ \text { W } & 0.0000000 & 0.0000000 & -0.0000000 \\ \text { C } & 0.0000000 & -2.0580351 & -0.0000000 \\ 0 & 0.0000000 & -3.1766053 & 0.0000000 \\ \text { C } & 0.0000000 & 0.0000000 & 2.0580351 \\ 0 & 0.0000000 & 0.0000000 & 3.1766053 \\ \text { C } & 2.0580351 & 0.0000000 & 0.0000000 \\ 0 & 3.1766053 & 0.0000000 & -0.0000000 \\ \text { C } & 0.0000000 & 0.0000000 & -2.0580351 \\ 0 & 0.0000000 & 0.0000000 & -3.1766053 \\ \text { C } & -2.0580351 & -0.0000000 & 0.0000000 \\ \text { O } & -3.1766053 & -0.0000000 & 0.0000000\end{array}$


16. Optimized Structures at the CAM-QTP-02 Level

The Cartesian coordinates of the structures are listed in Ångström.

\begin{tabular}{|c|c|c|c|}
\hline W & -0.0000000 & 0.0000000 & 0.0000000 \\
\hline 0 & -1.0187135 & 1.0187135 & 1.0187135 \\
\hline 0 & 1.0187135 & -1.0187135 & 1.0187135 \\
\hline 0 & 1.0187135 & 1.0187135 & -1.0187135 \\
\hline 0 & -1.0187135 & -1.0187135 & -1.0187135 \\
\hline W & -0.0006067 & 0.0013614 & -0.0897190 \\
\hline S & 0.0154315 & -0.0320115 & 2.1500234 \\
\hline 0 & 1.6381595 & 0.0043174 & -0.6986474 \\
\hline 0 & -0.8317347 & -1.4094174 & -0.7019642 \\
\hline 0 & -0.8212497 & 1.4357501 & -0.659692 \\
\hline $\mathrm{S}$ & -0.0160671 & 0.0315871 & 2.2708981 \\
\hline W & 0.0126422 & -0.0141638 & 0.0570796 \\
\hline $\mathrm{S}$ & -0.9999703 & 1.7599969 & -0.797827 \\
\hline 0 & 1.6558369 & -0.0563052 & -0.493220 \\
\hline 0 & -0.7842738 & -1.4505011 & -0.4965207 \\
\hline S & 0.0194274 & 0.0169935 & 2.2652937 \\
\hline W & -0.0342963 & -0.0188425 & 0.073332 \\
\hline $\mathrm{S}$ & -0.9859593 & -1.8618150 & -0.6385349 \\
\hline $\mathrm{S}$ & -1.0992320 & 1.7375227 & -0.694376 \\
\hline 0 & 1.5825643 & 0.0232633 & -0.513419 \\
\hline W & -0.0000000 & 0.0000000 & 0.0000000 \\
\hline S & -1.2551414 & 1.2551414 & 1.2551414 \\
\hline $\mathrm{S}$ & 1.2551414 & -1.2551414 & 1.255141 \\
\hline S & 1.2551414 & 1.2551414 & -1.255141 \\
\hline$S$ & -1.2551414 & -1.2551414 & -1.255141 \\
\hline $\mathrm{F}$ & 1.8178175 & 0.0000000 & -0.0000000 \\
\hline W & -0.0000000 & -0.0000000 & 0.000000 \\
\hline$F$ & 0.0000000 & -0.0000000 & -1.817817 \\
\hline $\mathrm{F}$ & -1.8178175 & -0.0000000 & 0.000000 \\
\hline $\mathrm{F}$ & -0.0000000 & 1.8178175 & -0.000000 \\
\hline $\mathrm{F}$ & 0.0000000 & 0.0000000 & 1.817817 \\
\hline $\mathrm{F}$ & 0.0000000 & -1.8178175 & 0.000000 \\
\hline W & 0.0000000 & -0.0000000 & 0.000000 \\
\hline $\mathrm{Cl}$ & 0.0000000 & & 0.000000 \\
\hline $\mathrm{Cl}$ & 0.0000000 & 0.0000000 & 2.266074 \\
\hline $\mathrm{Cl}$ & -2.2660744 & 0.0000000 & 0.000000 \\
\hline $\mathrm{Cl}$ & 0.0000000 & 0.0000000 & -2.266074 \\
\hline $\mathrm{Cl}$ & -0.0000000 & -2.2660744 & 0.0000000 \\
\hline $\mathrm{Cl}$ & 2.2660744 & -0.0000000 & 0.000000 \\
\hline
\end{tabular}




$\begin{array}{rrrr}\text { C } & -0.0000000 & 2.0497202 & 0.0000000 \\ \text { C } & -0.0000000 & 3.1751703 & -0.0000000 \\ \text { W } & -0.0000000 & -0.0000000 & -0.0000000 \\ \text { C } & 0.0000000 & -2.0497202 & 0.0000000 \\ 0 & 0.0000000 & -3.1751703 & -0.0000000 \\ \text { C } & -0.0000000 & -0.0000000 & 2.0497202 \\ 0 & 0.0000000 & -0.0000000 & 3.1751703 \\ \text { C } & 2.0497202 & 0.0000000 & 0.0000000 \\ 0 & 3.1751703 & 0.0000000 & -0.0000000 \\ \text { C } & -0.0000000 & -0.0000000 & -2.0497202 \\ 0 & 0.0000000 & 0.0000000 & -3.1751703 \\ \text { C } & -2.0497202 & -0.0000000 & 0.0000000 \\ 0 & -3.1751703 & -0.0000000 & 0.0000000\end{array}$


17. Optimized Structures at the LH07t-SVWN Level

The Cartesian coordinates of the structures are listed in Ångström.

\begin{tabular}{|c|c|c|c|}
\hline W & -0.0000000 & 0.0000000 & 0.0000000 \\
\hline 0 & -1.0272748 & 1.0272748 & 1.0272748 \\
\hline J & 1.0272748 & -1.0272748 & 1.0272748 \\
\hline 0 & 1.0272748 & 1.0272748 & -1.0272748 \\
\hline 0 & -1.0272748 & -1.0272748 & -1.0272748 \\
\hline W & -0.0005848 & 0.0013290 & -0.0874181 \\
\hline $\mathrm{S}$ & 0.0155384 & -0.0322047 & 2.1624893 \\
\hline 0 & 1.6532915 & 0.0043370 & -0.7036572 \\
\hline 0 & -0.8394125 & -1.4224024 & -0.7070293 \\
\hline 0 & -0.8288326 & 1.4489411 & -0.6643847 \\
\hline S & -0.0178568 & 0.0349609 & 2.2837434 \\
\hline W & 0.0124578 & -0.0139457 & 0.0572585 \\
\hline S & -1.0066140 & 1.7715374 & -0.7995937 \\
\hline 0 & 1.6734719 & -0.0562796 & -0.4987034 \\
\hline 0 & -0.7932909 & -1.4656590 & -0.5022959 \\
\hline $\mathrm{S}$ & 0.0168624 & 0.0171034 & 2.2805404 \\
\hline W & -0.0332469 & -0.0188537 & 0.0729496 \\
\hline $\mathrm{S}$ & -0.9951541 & -1.8738551 & -0.6420472 \\
\hline $\mathrm{S}$ & -1.1089900 & 1.7487824 & -0.698327 \\
\hline 0 & 1.6030325 & 0.0239449 & -0.5208191 \\
\hline W & -0.0000000 & 0.0000000 & 0.0000000 \\
\hline $\mathrm{S}$ & -1.2656386 & 1.2656386 & 1.265638 \\
\hline $\mathrm{S}$ & 1.2656386 & -1.2656386 & 1.265638 \\
\hline $\mathrm{S}$ & 1.2656386 & 1.2656386 & -1.2656386 \\
\hline S & -1.2656386 & -1.2656386 & -1.265638 \\
\hline $\mathrm{F}$ & 1.8369249 & -0.0000000 & 0.0000000 \\
\hline W & 0.0000000 & 0.0000000 & 0.000000 \\
\hline $\mathrm{F}$ & 0.0000000 & 0.0000000 & -1.836924 \\
\hline $\mathrm{F}$ & -1.8369249 & 0.0000000 & 0.000000 \\
\hline $\mathrm{F}$ & 0.0000000 & 1.8369249 & 0.000000 \\
\hline $\mathrm{F}$ & 0.0000000 & 0.0000000 & 1.836924 \\
\hline $\mathrm{F}$ & -0.0000000 & -1.8369249 & -0.000000 \\
\hline W & 0.0000000 & -0.0000000 & -0.000000 \\
\hline $\mathrm{Cl}$ & 0.0000000 & 2.2903526 & -0.000000 \\
\hline $\mathrm{Cl}$ & 0.0000000 & 0.0000000 & 2.290352 \\
\hline $\mathrm{Cl}$ & -2.2903526 & 0.0000000 & 0.000000 \\
\hline $\mathrm{Cl}$ & 0.0000000 & 0.0000000 & -2.290352 \\
\hline $\mathrm{Cl}$ & -0.0000000 & -2.2903526 & -0.000000 \\
\hline $\mathrm{Cl}$ & 2.2903526 & -0.0000000 & -0.000000 \\
\hline
\end{tabular}




$\begin{array}{rrrr}\text { C } & -0.0000000 & 2.0492480 & 0.0000000 \\ \text { C } & -0.0000000 & 3.1915742 & -0.0000000 \\ \text { W } & 0.0000000 & -0.0000000 & -0.0000000 \\ \text { C } & 0.0000000 & -2.0492480 & -0.0000000 \\ 0 & 0.0000000 & -3.1915742 & -0.0000000 \\ \text { C } & -0.0000000 & -0.0000000 & 2.0492480 \\ 0 & 0.0000000 & -0.0000000 & 3.1915742 \\ \text { C } & 2.0492480 & 0.0000000 & 0.0000000 \\ 0 & 3.1915742 & 0.0000000 & 0.0000000 \\ \text { C } & -0.0000000 & -0.0000000 & -2.0492480 \\ 0 & 0.0000000 & -0.0000000 & -3.1915742 \\ \text { C } & -2.0492480 & -0.0000000 & -0.0000000 \\ 0 & -3.1915742 & -0.0000000 & 0.0000000\end{array}$


18. Optimized Structures at the LH12ct-SsirPW92 Level

The Cartesian coordinates of the structures are listed in Ångström.

\begin{tabular}{|c|c|c|c|}
\hline W & -0.0000000 & -0.0000000 & -0.0000000 \\
\hline 0 & -1.0253598 & 1.0253598 & 1.0253598 \\
\hline 0 & 1.0253598 & -1.0253598 & 1.0253598 \\
\hline 0 & 1.0253598 & 1.0253598 & -1.0253598 \\
\hline 0 & -1.0253598 & -1.0253598 & -1.0253598 \\
\hline W & -0.0005916 & 0.0013428 & -0.0879829 \\
\hline $\mathrm{S}$ & 0.0155297 & -0.0322013 & 2.1613952 \\
\hline 0 & 1.6495300 & 0.0043458 & -0.7030790 \\
\hline 0 & -0.8375128 & -1.4191594 & -0.7064300 \\
\hline 0 & -0.8269553 & 1.4456721 & -0.6639033 \\
\hline S & -0.0174165 & 0.0341855 & 2.2819854 \\
\hline W & 0.0126189 & -0.0141782 & 0.0570729 \\
\hline $\mathrm{S}$ & -1.0056674 & 1.7698092 & -0.7996874 \\
\hline 0 & 1.6693610 & -0.0568531 & -0.497687 \\
\hline 0 & -0.7907279 & -1.4623494 & -0.501274 \\
\hline $\mathrm{S}$ & 0.0174690 & 0.0170191 & 2.2779350 \\
\hline W & -0.0330490 & -0.0188265 & 0.0728766 \\
\hline $\mathrm{S}$ & -0.9934662 & -1.8718586 & -0.6414720 \\
\hline $\mathrm{S}$ & -1.1071623 & 1.7469258 & -0.6977838 \\
\hline 0 & 1.5987124 & 0.0238622 & -0.5192597 \\
\hline W & 0.0000000 & -0.0000000 & -0.0000000 \\
\hline S & -1.2637204 & 1.2637204 & 1.2637204 \\
\hline $\mathrm{S}$ & 1.2637204 & -1.2637204 & 1.263720 \\
\hline $\mathrm{S}$ & 1.2637204 & 1.2637204 & -1.263720 \\
\hline$S$ & -1.2637204 & -1.2637204 & -1.263720 \\
\hline $\mathrm{F}$ & 1.8347221 & 0.0000000 & -0.0000000 \\
\hline W & -0.0000000 & -0.0000 & -0.000000 \\
\hline F & 0.0000000 & 0.0 & -1.834722 \\
\hline $\mathrm{F}$ & -1.8347221 & -0.0000000 & -0.000000 \\
\hline $\mathrm{F}$ & -0.0000000 & 1.8347221 & -0.000000 \\
\hline $\mathrm{F}$ & 0.0000000 & 0.0000000 & 1.834722 \\
\hline $\mathrm{F}$ & 0.0000000 & -1.8347221 & 0.000000 \\
\hline W & -0.0000000 & -0.0000000 & \\
\hline $\mathrm{Cl}$ & -0.0000000 & 2.2876424 & -0.000000 \\
\hline $\mathrm{Cl}$ & 0.0000000 & -0.0000000 & 2.287642 \\
\hline $\mathrm{Cl}$ & -2.2876424 & -0.0000000 & 0.000000 \\
\hline $\mathrm{Cl}$ & 0.0000000 & 0.0000000 & -2.2876424 \\
\hline $\mathrm{Cl}$ & 0.0000000 & -2.2876424 & -0.000000 \\
\hline $\mathrm{Cl}$ & 2.2876424 & 0.0000000 & 0.000000 \\
\hline
\end{tabular}




$\begin{array}{rrrr}\text { C } & -0.0000000 & 2.0480934 & -0.0000000 \\ \text { C } & -0.0000000 & 3.1896620 & -0.0000000 \\ \text { W } & 0.0000000 & 0.0000000 & 0.0000000 \\ \text { C } & 0.0000000 & -2.0480934 & -0.0000000 \\ 0 & 0.0000000 & -3.1896620 & -0.0000000 \\ \text { C } & 0.0000000 & 0.0000000 & 2.0480934 \\ 0 & -0.0000000 & 0.0000000 & 3.1896620 \\ \text { C } & 2.0480934 & 0.0000000 & -0.0000000 \\ 0 & 3.1896620 & 0.0000000 & -0.0000000 \\ \text { C } & 0.0000000 & 0.0000000 & -2.0480934 \\ 0 & -0.0000000 & 0.0000000 & -3.1896620 \\ \text { C } & -2.0480934 & -0.0000000 & 0.0000000 \\ \text { D } & -3.1896620 & -0.0000000 & 0.0000000\end{array}$


19. Optimized Structures at the LH14t-calPBE Level

The Cartesian coordinates of the structures are listed in Ångström.

\begin{tabular}{|c|c|c|c|}
\hline W & -0.0000000 & 0.0000000 & -0.0000000 \\
\hline 0 & -1.0270219 & 1.0270219 & 1.0270219 \\
\hline 0 & 1.0270219 & -1.0270219 & 1.0270219 \\
\hline 0 & 1.0270219 & 1.0270219 & -1.0270219 \\
\hline 0 & -1.0270219 & -1.0270219 & -1.0270219 \\
\hline W & -0.0005611 & 0.0013287 & -0.0878040 \\
\hline S & 0.0154632 & -0.0321330 & 2.1600725 \\
\hline 0 & 1.6535472 & 0.0042996 & -0.7027850 \\
\hline 0 & -0.8395038 & -1.4226402 & -0.7061014 \\
\hline 0 & -0.8289454 & 1.4491449 & -0.663382 \\
\hline $\mathrm{S}$ & -0.0187146 & 0.0363386 & 2.2815122 \\
\hline W & 0.0128198 & -0.0145613 & 0.0568155 \\
\hline $\mathrm{S}$ & -1.0058749 & 1.7703361 & -0.7972043 \\
\hline 0 & 1.6740635 & -0.0556164 & -0.4986255 \\
\hline 0 & -0.7941259 & -1.4658830 & -0.502088 \\
\hline S & 0.0152744 & 0.0170771 & 2.2787673 \\
\hline W & -0.0322191 & -0.0188006 & 0.072562 \\
\hline $\mathrm{S}$ & -0.9954762 & -1.8720251 & -0.640766 \\
\hline S & -1.1093245 & 1.7470122 & -0.696905 \\
\hline 0 & 1.6042494 & 0.0238583 & -0.521362 \\
\hline W & -0.0000000 & 0.0000000 & 0.0000000 \\
\hline $\mathrm{S}$ & -1.2649616 & 1.2649616 & 1.2649616 \\
\hline $\mathrm{S}$ & 1.2649616 & -1.2649616 & 1.2649616 \\
\hline S & 1.2649616 & 1.2649616 & -1.264961 \\
\hline$S$ & -1.2649616 & -1.2649616 & -1.264961 \\
\hline $\mathrm{F}$ & 1.8352415 & 0.0000000 & -0.0000000 \\
\hline W & 0.0000000 & 0.0000000 & -0.0000000 \\
\hline $\mathrm{F}$ & 0.0000000 & -0.00 & -1.835241 \\
\hline $\mathrm{F}$ & -1.8352415 & -0.0000000 & -0.000000 \\
\hline $\mathrm{F}$ & -0.0000000 & 1.8352415 & -0.000000 \\
\hline $\mathrm{F}$ & 0.0000000 & -0.0000000 & 1.835241 \\
\hline $\mathrm{F}$ & 0.0000000 & -1.8352415 & 0.000000 \\
\hline W & -0.0000000 & -0.0000000 & 0.000000 \\
\hline $\mathrm{Cl}$ & -0.0000000 & & -0.000000 \\
\hline $\mathrm{Cl}$ & 0.0000000 & 0.0000000 & 2.286595 \\
\hline $\mathrm{Cl}$ & -2.2865955 & -0.0000000 & 0.000000 \\
\hline $\mathrm{Cl}$ & 0.0000000 & 0.0000000 & -2.286595 \\
\hline $\mathrm{Cl}$ & 0.0000000 & -2.2865955 & -0.0000000 \\
\hline $\mathrm{Cl}$ & 2.2865955 & 0.0000000 & -0.000000 \\
\hline
\end{tabular}




$\begin{array}{rrrr}\text { C } & -0.0000000 & 2.0484190 & -0.0000000 \\ \text { C } & -0.0000000 & 3.1891341 & 0.0000000 \\ \text { W } & -0.0000000 & -0.0000000 & 0.0000000 \\ \text { C } & 0.0000000 & -2.0484190 & 0.0000000 \\ 0 & 0.0000000 & -3.1891341 & -0.0000000 \\ \text { C } & -0.0000000 & 0.0000000 & 2.0484190 \\ 0 & 0.0000000 & 0.0000000 & 3.1891341 \\ \text { C } & 2.0484190 & 0.0000000 & -0.0000000 \\ 0 & 3.1891341 & 0.0000000 & 0.0000000 \\ \text { C } & -0.0000000 & 0.0000000 & -2.0484190 \\ 0 & 0.0000000 & 0.0000000 & -3.1891341 \\ \text { C } & -2.0484190 & -0.0000000 & 0.0000000 \\ 0 & -3.1891341 & -0.0000000 & 0.0000000\end{array}$


20. Optimized Structures at the LH2Ot Level

The Cartesian coordinates of the structures are listed in Ångström.

\begin{tabular}{|c|c|c|c|}
\hline W & -0.0000000 & 0.0000000 & 0.0000000 \\
\hline 0 & -1.0235283 & 1.0235283 & 1.0235283 \\
\hline 0 & 1.0235283 & -1.0235283 & 1.0235283 \\
\hline 0 & 1.0235283 & 1.0235283 & -1.023528 \\
\hline 0 & -1.0235283 & -1.0235283 & -1.023528 \\
\hline W & -0.0005572 & 0.0013059 & -0.087740 \\
\hline S & 0.0154041 & -0.0319233 & 2.150286 \\
\hline 0 & 1.6484423 & 0.0042416 & -0.699493 \\
\hline 0 & -0.8368957 & -1.4182431 & -0.702878 \\
\hline 0 & -0.8263935 & 1.4446190 & -0.660173 \\
\hline S & -0.0192910 & 0.0374810 & 2.271740 \\
\hline W & 0.0128118 & -0.0146607 & 0.056734 \\
\hline S & -1.0016974 & 1.7631025 & -0.792043 \\
\hline 0 & 1.6688314 & -0.0544314 & -0.496244 \\
\hline 0 & -0.7924869 & -1.4608775 & -0.499778 \\
\hline S & 0.0133260 & 0.0168551 & 2.269183 \\
\hline W & -0.0318243 & -0.0187160 & 0.072439 \\
\hline S & -0.9923924 & -1.8634982 & -0.636829 \\
\hline S & -1.1059093 & 1.7389093 & -0.692712 \\
\hline 0 & 1.5993040 & 0.0235718 & -0.519786 \\
\hline W & -0.0000000 & 0.0000000 & 0.000000 \\
\hline$S$ & -1.2594868 & 1.2594868 & 1.259486 \\
\hline S & 1.2594868 & -1.2594868 & 1.259486 \\
\hline $\mathrm{S}$ & 1.2594868 & 1.2594868 & -1.259486 \\
\hline$S$ & -1.2594868 & -1.2594868 & -1.259486 \\
\hline F & 1.8286498 & & 0.000000 \\
\hline W & -0.0000000 & -0.0000000 & 0.000000 \\
\hline F & 0.0000000 & 0.0000000 & -1.828649 \\
\hline F & -1.8286498 & -0.0000000 & -0.000000 \\
\hline $\mathrm{F}$ & -0.0000000 & 1.8286498 & 0.000000 \\
\hline$F$ & 0.0000000 & 0.0000000 & 1.82864 \\
\hline $\mathrm{F}$ & 0.0000000 & -1.8286498 & 0.00000 \\
\hline W & -0.0000000 & -0.0000000 & -0.000000 \\
\hline $\mathrm{Cl}$ & -0.0000000 & 2.2759758 & -0.000000 \\
\hline $\mathrm{Cl}$ & 0.0000000 & 0.0000000 & 2.27597 \\
\hline $\mathrm{Cl}$ & -2.2759758 & -0.0000000 & 0.000000 \\
\hline $\mathrm{Cl}$ & 0.0000000 & 0.0000000 & -2.27597 \\
\hline $\mathrm{Cl}$ & 0.0000000 & -2.2759758 & -0.000000 \\
\hline $\mathrm{Cl}$ & 2.2759758 & 0.0000000 & 0.000000 \\
\hline
\end{tabular}




$\begin{array}{rrrr}\text { C } & -0.0000000 & 2.0479972 & -0.0000000 \\ \text { C } & -0.0000000 & 3.1837793 & 0.0000000 \\ \text { W } & -0.0000000 & -0.0000000 & 0.0000000 \\ \text { C } & 0.0000000 & -2.0479972 & -0.0000000 \\ 0 & 0.0000000 & -3.1837793 & -0.0000000 \\ \text { C } & -0.0000000 & 0.0000000 & 2.0479972 \\ 0 & 0.0000000 & 0.0000000 & 3.1837793 \\ \text { C } & 2.0479972 & 0.0000000 & 0.0000000 \\ 0 & 3.1837793 & 0.0000000 & -0.0000000 \\ \text { C } & -0.0000000 & 0.0000000 & -2.0479972 \\ 0 & 0.0000000 & 0.0000000 & -3.1837793 \\ \text { C } & -2.0479972 & -0.0000000 & 0.0000000 \\ 0 & -3.1837793 & -0.0000000 & 0.0000000\end{array}$




\section{Optimized Structures at the mPSTS Level}

The Cartesian coordinates of the structures are listed in Ångström.

\begin{tabular}{|c|c|c|c|}
\hline W & -0.0000000 & 0.0000000 & 0.0000000 \\
\hline 0 & -1.0324845 & 1.0324845 & 1.0324845 \\
\hline 0 & 1.0324845 & -1.0324845 & 1.0324845 \\
\hline 0 & 1.0324845 & 1.0324845 & -1.0324845 \\
\hline 0 & -1.0324845 & -1.0324845 & -1.0324845 \\
\hline W & -0.0005872 & 0.0013174 & -0.0870629 \\
\hline $\mathrm{S}$ & 0.0156198 & -0.0323364 & 2.1728229 \\
\hline 0 & 1.6607682 & 0.0043517 & -0.7072680 \\
\hline 0 & -0.8432092 & -1.4288356 & -0.710685 \\
\hline 0 & -0.8325916 & 1.4555030 & -0.6678067 \\
\hline S & -0.0177340 & 0.0347939 & 2.293252 \\
\hline W & 0.0122297 & -0.0135963 & 0.0575230 \\
\hline $\mathrm{S}$ & -1.0108517 & 1.7789809 & -0.8036611 \\
\hline 0 & 1.6806726 & -0.0572037 & -0.5015228 \\
\hline 0 & -0.7961486 & -1.4723608 & -0.5051828 \\
\hline $\mathrm{S}$ & 0.0173464 & 0.0172652 & 2.2892851 \\
\hline W & -0.0334727 & -0.0188323 & 0.0730111 \\
\hline $\mathrm{S}$ & -0.9986371 & -1.8813012 & -0.6450182 \\
\hline S & -1.1129982 & 1.7559177 & -0.701501 \\
\hline 0 & 1.6102657 & 0.0240726 & -0.523480 \\
\hline W & -0.0000000 & 0.0000000 & -0.000000 \\
\hline S & -1.2705604 & 1.2705604 & 1.270560 \\
\hline$S$ & 1.2705604 & -1.2705604 & 1.270560 \\
\hline $\mathrm{S}$ & 1.2705604 & 1.2705604 & -1.270560 \\
\hline $\mathrm{S}$ & -1.2705604 & -1.2705604 & -1.270560 \\
\hline F & 1.8419230 & & -0.000000 \\
\hline W & -0.0000000 & -0.0000000 & -0.0000000 \\
\hline $\mathrm{F}$ & 0.0000000 & 0.0000000 & -1.841923 \\
\hline $\mathrm{F}$ & -1.8419230 & -0.0000000 & -0.000000 \\
\hline $\mathrm{F}$ & -0.0000000 & 1.8419230 & 0.000000 \\
\hline $\mathrm{F}$ & 0.0000000 & 0.0000000 & 1.841923 \\
\hline $\mathrm{F}$ & 0.0000000 & -1.8419230 & -0.000000 \\
\hline $\mathrm{F}$ & 1.8438640 & 0.0000000 & 0.000000 \\
\hline W & -0.0000000 & -0.0000000 & 0.000000 \\
\hline $\mathrm{Cl}$ & -0.0000000 & & 0.000000 \\
\hline $\mathrm{Cl}$ & 0.0000000 & -0.0000000 & 2.294509 \\
\hline $\mathrm{Cl}$ & -2.2945094 & -0.0000000 & 0.000000 \\
\hline $\mathrm{Cl}$ & 0.0000000 & -0.0000000 & -2.294509 \\
\hline $\mathrm{Cl}$ & 0.0000000 & -2.2945094 & -0.0000000 \\
\hline $\mathrm{Cl}$ & 2.2945094 & 0.0000000 & 0.000000 \\
\hline
\end{tabular}




$\begin{array}{rrrr}\text { C } & -0.0000000 & 2.0589242 & 0.0000000 \\ \text { C } & -0.0000000 & 3.2041063 & 0.0000000 \\ \text { W } & -0.0000000 & -0.0000000 & -0.0000000 \\ \text { C } & 0.0000000 & -2.0589242 & -0.0000000 \\ 0 & 0.0000000 & -3.2041063 & 0.0000000 \\ \text { C } & 0.0000000 & -0.0000000 & 2.0589242 \\ 0 & -0.0000000 & -0.0000000 & 3.2041063 \\ \text { C } & 2.0589242 & 0.0000000 & -0.0000000 \\ 0 & 3.2041063 & 0.0000000 & 0.0000000 \\ \text { C } & 0.0000000 & -0.0000000 & -2.0589242 \\ 0 & 0.0000000 & -0.0000000 & -3.2041063 \\ \text { C } & -2.0589242 & -0.0000000 & 0.0000000 \\ 0 & -3.2041063 & -0.0000000 & 0.0000000\end{array}$


The Cartesian coordinates of the structures are listed in Ångström.

\begin{tabular}{|c|c|c|c|}
\hline W & 0.0000000 & 0.0000000 & 0.0000000 \\
\hline 0 & -1.0287821 & 1.0287821 & 1.0287821 \\
\hline 0 & 1.0287821 & -1.0287821 & 1.0287821 \\
\hline 0 & 1.0287821 & 1.0287821 & -1.0287821 \\
\hline 0 & -1.0287821 & -1.0287821 & -1.0287821 \\
\hline W & -0.0005472 & 0.0012965 & -0.0857249 \\
\hline S & 0.0154263 & -0.0320046 & 2.1499114 \\
\hline 0 & 1.6584223 & 0.0042630 & -0.7000855 \\
\hline 0 & -0.8419568 & -1.4268278 & -0.7034504 \\
\hline 0 & -0.8313445 & 1.4532728 & -0.660650 \\
\hline $\mathrm{S}$ & -0.0212022 & 0.0408434 & 2.2724263 \\
\hline W & 0.0123041 & -0.0136994 & 0.0574385 \\
\hline $\mathrm{S}$ & -1.0028696 & 1.7649944 & -0.7887947 \\
\hline 0 & 1.6791383 & -0.0527493 & -0.4985278 \\
\hline 0 & -0.7992027 & -1.4687750 & -0.502133 \\
\hline S & 0.0105761 & 0.0168041 & 2.2714594 \\
\hline W & -0.0323497 & -0.0188175 & 0.072607 \\
\hline $\mathrm{S}$ & -0.9961803 & -1.8646331 & -0.636233 \\
\hline $\mathrm{S}$ & -1.1095130 & 1.7397146 & -0.692164 \\
\hline 0 & 1.6099709 & 0.0240540 & -0.523373 \\
\hline W & 0.0000000 & -0.0000000 & -0.0000000 \\
\hline S & -1.2616803 & 1.2616803 & 1.2616803 \\
\hline $\mathrm{S}$ & 1.2616803 & -1.2616803 & 1.2616803 \\
\hline S & 1.2616803 & 1.2616803 & -1.261680 \\
\hline$S$ & -1.2616803 & -1.2616803 & -1.261680 \\
\hline $\mathrm{F}$ & 1.8378936 & -0.0000000 & -0.0000000 \\
\hline W & -0.0000000 & -0.0000000 & 0.000000 \\
\hline$F$ & 0.0000000 & -0.0000000 & -1.837893 \\
\hline $\mathrm{F}$ & -1.8378936 & 0.0000000 & -0.000000 \\
\hline $\mathrm{F}$ & 0.0000000 & 1.8378936 & 0.000000 \\
\hline $\mathrm{F}$ & 0.0000000 & -0.0000000 & 1.837893 \\
\hline $\mathrm{F}$ & -0.0000000 & -1.8378936 & 0.000000 \\
\hline W & -0.0000000 & -0.0000000 & 0.000000 \\
\hline $\mathrm{Cl}$ & & & 0.000000 \\
\hline $\mathrm{Cl}$ & 0.0000000 & 0.0000000 & 2.277014 \\
\hline $\mathrm{Cl}$ & -2.2770148 & 0.0000000 & -0.000000 \\
\hline $\mathrm{Cl}$ & 0.0000000 & 0.0000000 & -2.277014 \\
\hline $\mathrm{Cl}$ & -0.0000000 & -2.2770148 & 0.0000000 \\
\hline $\mathrm{Cl}$ & 2.2770148 & -0.0000000 & -0.000000 \\
\hline
\end{tabular}




$\begin{array}{rrrr}\text { C } & -0.0000000 & 2.0422253 & -0.0000000 \\ \text { O } & -0.0000000 & 3.1820801 & 0.0000000 \\ \text { W } & 0.0000000 & 0.0000000 & 0.0000000 \\ \text { C } & 0.0000000 & -2.0422253 & 0.0000000 \\ 0 & 0.0000000 & -3.1820801 & -0.0000000 \\ \text { C } & 0.0000000 & 0.0000000 & 2.0422253 \\ 0 & 0.0000000 & 0.0000000 & 3.1820801 \\ \text { C } & 2.0422253 & 0.0000000 & 0.0000000 \\ 0 & 3.1820801 & 0.0000000 & 0.0000000 \\ \text { C } & 0.0000000 & 0.0000000 & -2.0422253 \\ 0 & -0.0000000 & 0.0000000 & -3.1820801 \\ \text { C } & -2.0422253 & -0.0000000 & -0.0000000 \\ \text { D } & -3.1820801 & -0.0000000 & 0.0000000\end{array}$




\section{S6. MAGNETICALLY INDUCED CURRENT DENSITIES}

Magnetically induced current densities were calculated with the gauge-including magnetically induced current (GIMIC) method [46-48, 115]. Current strengths are obtained by numerical integration along a plane. Structures were taken from Ref. 116 The KT3 functional is not considered for $\left[\mathrm{Th} @ \mathrm{Bi}_{12}\right]^{4-}$ and related compounds as it was designed for the NMR shieldings of light main-group elements [68] and did not improve upon the other generalized gradient approximations for heavy elements in Sec. S5 The def2-TZVP orbital [15] and auxiliary basis sets [30] together with the def-TZVP orbital [117] and auxiliary basis sets were employed for the effective-core potential (ECP) [117, 118] calculations whereas the DLU-X2C Hamiltonian is combined with the uncontracted Dyall-VTZ [119,-121], Jorge-TZP-DKH [62, 63, 122, 123], and x2c-XVPall-s $(X=S, T Z, Q Z)$ [99, 124] bases. The RI- $J$ approximation was used in all calculations involving Karlsruhe basis sets. Tight SCF thresholds of $10^{-8} \mathrm{E}_{\mathrm{h}}$ and a threshold of $10^{-7}$ a.u. were chosen for the residuum of the coupled perturbed Kohn-Sham equations. COSMO was applied to compensate the negative charges. Medium sized grids (gridsize 3 for ECP calculations and gridsize 3a for DLU$\mathrm{X} 2 \mathrm{C}$ calculations) were employed. More than 3,500 basis functions (spherical atomic orbital basis) are used for [Th@ $\left.\mathrm{Bi}_{12}\right]^{4-}$ with the Dyall-VTZ basis.

Furthermore, nucleus-independent chemical shifts (NICS) [125] were evaluated for the bare $\mathrm{Bi}_{12}$ clusters. Both GIMIC and NICS can be used to study the aromaticity based on the magnetic criterion [48, 126-128].

TABLE S85. Current strengths in nA/T of $\left[\mathrm{Th} @ \mathrm{Bi}_{12}\right]^{4-}$ with various computational methods. The BP86, PBE, TPSS, and TPSSh results are taken from Ref. 116. The inclusion of the current density to restore gauge-origin invariance instead of the external vector potential in the generalized kinetic energy is denoted by a $\mathrm{c}$ at the beginning of the functional name.

\begin{tabular}{lccc}
\hline & def(2)-TZVP/ECP & Dyall-VTZ/X2C & Jorge-DKH-TZP/X2C \\
\hline BP86 & 22.4 & 23.2 & 24.7 \\
PBE & 22.7 & 23.4 & 24.8 \\
TPSS & 22.9 & 23.3 & 24.8 \\
cTPSS & 23.1 & 23.3 & 24.9 \\
B3LYP & 24.1 & 24.6 & 25.9 \\
PBE0 & 25.1 & 25.4 & 25.5 \\
TPSSh & 24.0 & 24.2 & 25.5 \\
cTPSSh & 24.1 & 24.4 & 25.6 \\
LC- $\omega$ PBE & 26.5 & 25.9 & 27.5 \\
$\omega B 97 X-D$ & 25.8 & 25.9 & 27.4 \\
CAM-B3LYP & 25.3 & 25.3 & 26.8 \\
LH07t & 23.9 & 26.2 & 24.7 \\
cLH07t & 24.0 & 24.7 & 25.8 \\
LH12ct & 24.5 & 25.3 & 26.2 \\
cLH12ct & 24.6 & 25.4 & 26.2 \\
LH14t & 24.0 & 24.9 & 26.0 \\
cLH14t & 24.1 & 24.9 & 26.0 \\
LH20t & 25.1 & 25.4 & 26.4 \\
cLH20t & 24.9 & 25.4 & 26.3 \\
mPSTS & 24.0 & 24.4 & 25.6 \\
cmPSTS & 24.2 & 24.6 & 25.6 \\
LHJ14 & 24.2 & 24.7 & 26.5 \\
cLHJ14 & 23.4 & 23.8 & 26.0 \\
\hline
\end{tabular}


TABLE S86. Current strengths (in nA/T) and NICS (in ppm) of $\mathrm{Bi}_{12}^{8-}$ with various computational methods. The BP86, PBE, TPSS, and TPSSh results as well as the results with B3LYP, PBE0, and the CAM-B3LYP hybrid functional of the x2c-type basis sets are taken from Ref. 116

\begin{tabular}{|c|c|c|c|c|c|c|c|c|c|c|c|}
\hline & \multirow{2}{*}{$\frac{\operatorname{def}(2)-\mathrm{TZVP}}{\text { GIMIC NICS }}$} & \multicolumn{2}{|c|}{ Dyall-VTZ } & \multicolumn{2}{|c|}{ Jorge-DKH-TZP } & \multicolumn{2}{|c|}{ x2c-SVPall-s } & \multicolumn{2}{|c|}{ x2c-TZVPall-s } & \multicolumn{2}{|c|}{ x2c-QZVPall-s } \\
\hline & & GIMIC & NICS & GIMIC & NICS & GIMIC & NICS & GIMIC & NICS & GIMIC & NICS \\
\hline BP86 & $23.4-16.8$ & 23.3 & -17.6 & 23.0 & -15.3 & 21.0 & -15.5 & 21.8 & -16.3 & 23.0 & -18.0 \\
\hline PBE & $23.7-17.1$ & 23.4 & -17.8 & 23.2 & -15.4 & 21.1 & -15.6 & 22.0 & -16.5 & 22.8 & -18.2 \\
\hline TPSS & $23.4-17.1$ & 23.1 & -17.7 & 22.8 & -15.7 & 20.8 & -15.7 & 21.7 & -16.5 & 23.1 & -18.0 \\
\hline cTPSS & $24.0-16.1$ & 23.3 & -17.9 & 22.9 & -15.8 & 20.9 & -15.8 & 21.9 & 16.7 & 23.0 & -18.2 \\
\hline B3LYP & $23.6-17.0$ & 23.4 & -17.6 & 23.0 & -15.3 & 20.8 & -16.3 & 21.9 & -16.6 & 23.1 & -17.9 \\
\hline PBE0 & $24.2-17.9$ & 23.1 & -17.7 & 23.4 & -16.9 & 21.2 & -15.6 & 22.3 & -17.3 & 23.6 & -18.7 \\
\hline TPSSh & $23.7-17.5$ & 23.4 & -18.0 & 23.0 & -16.3 & 20.9 & -16.2 & 21.9 & -16.9 & 23.1 & -18.3 \\
\hline cTPSSh & $23.8-17.6$ & 23.6 & -18.2 & 23.1 & -16.3 & 21.0 & -16.3 & 22.1 & -17.0 & 23.2 & -18.5 \\
\hline LC- $\omega$ PBE & $23.1-17.4$ & 22.8 & -17.6 & 22.0 & -17.2 & 19.6 & -16.2 & 21.2 & -16.7 & 22.5 & -17.7 \\
\hline$\omega \mathrm{B} 97 \mathrm{X}-\mathrm{D}$ & $23.6-17.3$ & 23.6 & -17.7 & 22.9 & -17.1 & 20.4 & -16.6 & 21.8 & -16.8 & 23.3 & -17.9 \\
\hline CAM-B3LYP & $23.2-16.9$ & 23.0 & -17.3 & 22.3 & -16.8 & 20.2 & -16.4 & 21.4 & -16.5 & 22.8 & -17.5 \\
\hline LH07t-S & $23.7-17.1$ & 23.0 & -16.4 & 23.5 & -17.8 & 20.8 & -16.3 & 21.9 & -16.7 & 23.3 & -18.1 \\
\hline cLH07t-SVWN & $23.8-17.2$ & 23.6 & -17.9 & 23.1 & -16.4 & 21.0 & -16.4 & 22.1 & -16.8 & 23.4 & -18.2 \\
\hline LH12ct-SsirPW92 & $23.9-17.3$ & 23.7 & -17.9 & 23.3 & -16.7 & 20.9 & -16.5 & 22.1 & -16.9 & 23.5 & -18.2 \\
\hline cLH12ct-SsirPW92 & $23.9-17.3$ & 23.8 & -17.9 & 23.3 & -16.7 & 21.1 & -16.5 & 22.2 & -16.9 & 23.5 & -18.2 \\
\hline LH14t-calPBE & $23.9-17.4$ & 23.7 & -18.0 & 23.2 & -16.5 & 21.0 & -16.5 & 22.1 & -16.9 & 23.5 & -18.4 \\
\hline cLH14t-calPBE & $23.8-17.4$ & 23.7 & -18.0 & 23.2 & -16.5 & 21.0 & -16.4 & 22.1 & -16.9 & 23.4 & -18.4 \\
\hline LH20t & $23.9-17.5$ & 23.8 & -18.1 & 23.4 & -16.9 & 20.9 & -16.5 & 22.0 & -16.9 & 23.7 & -18.5 \\
\hline cLH20t & $23.6-17.4$ & 23.5 & -18.1 & 23.2 & -16.8 & 20.6 & -16.5 & 21.7 & -16.8 & 23.3 & -18.4 \\
\hline mPSTS & $23.7-17.4$ & 23.5 & -18.0 & 23.0 & -16.4 & 20.9 & -16.3 & 22.0 & -16.9 & 23.1 & -18.3 \\
\hline cmPSTS & $23.8-17.6$ & 23.6 & -18.2 & 23.1 & -16.4 & 21.1 & -16.4 & 22.2 & -17.1 & 23.2 & -18.4 \\
\hline LHJ14 & $24.1-17.7$ & 24.2 & -18.4 & 23.3 & -17.2 & 21.1 & -16.5 & 22.2 & -16.8 & 24.1 & -18.8 \\
\hline cLHJ14 & $23.2-17.6$ & 23.1 & -18.2 & 22.7 & -17.1 & 20.4 & -16.5 & 21.3 & -16.8 & 23.0 & -18.6 \\
\hline
\end{tabular}


TABLE S87. Current strengths (in $\mathrm{nA} / \mathrm{T}$ ) and NICS (in ppm) of $\mathrm{Bi}_{12}^{12-}$ with various computational methods. The BP86, PBE, TPSS, and TPSSh results as well as the results with B3LYP, PBE0, and the CAM-B3LYP hybrid functional of the $\mathrm{x} 2 \mathrm{c}$-type basis sets are taken from Ref. 116 .

\begin{tabular}{|c|c|c|c|c|c|c|c|c|c|c|c|}
\hline & \multirow{2}{*}{$\frac{\operatorname{def}(2)-\mathrm{TZVP}}{\text { GIMIC NICS }}$} & \multicolumn{2}{|c|}{ Dyall-VTZ } & \multicolumn{2}{|c|}{ Jorge-DKH-TZP } & \multicolumn{2}{|c|}{ x2c-SVPall-s } & \multicolumn{2}{|c|}{ x2c-TZVPall-s } & \multicolumn{2}{|c|}{ x2c-QZVPall-s } \\
\hline & & GIMIC & NICS & GIMIC & NICS & GIMIC & NICS & GIMIC & NICS & GIMIC & NICS \\
\hline BP86 & $42.0-33.4$ & 41.1 & -34.2 & 41.0 & -32.1 & 36.2 & -31.3 & 37.9 & -32.6 & 40.4 & -34.7 \\
\hline PBE & $42.9-34.0$ & 41.9 & -34.8 & 41.8 & -32.7 & 36.9 & -31.7 & 38.7 & -33.2 & 41.1 & -35.3 \\
\hline TPSS & $42.6-33.9$ & 41.7 & -34.7 & 41.8 & -33.5 & 36.5 & -31.6 & 38.6 & -33.1 & 40.9 & -35.2 \\
\hline cTPSS & $42.8-34.3$ & 41.9 & -35.1 & 42.0 & -33.6 & 36.7 & -31.8 & 38.8 & -33.4 & 41.2 & -35.5 \\
\hline B3LYP & $0.7-33.2$ & 39.6 & -33.8 & 40.0 & -32.1 & 35.1 & -30.9 & 36.6 & -32.3 & 38.9 & -34.3 \\
\hline PBE0 & $3.0-34.8$ & 41.7 & -35.6 & 42.1 & -33.8 & 36.7 & -32.2 & 38.7 & -33.9 & 41.0 & -36.1 \\
\hline TPSSh & $42.8-34.3$ & 41.6 & -35.1 & 41.8 & -33.5 & 36.5 & -31.9 & 38.6 & -33.5 & 40.9 & -35.6 \\
\hline cTPSSh & $43.0-34.7$ & 41.9 & -35.4 & 42.0 & -33.6 & 36.7 & -32.1 & 38.8 & -33.7 & 41.1 & -35.8 \\
\hline LC- $\omega$ PBE & $39.6-33.5$ & 38.3 & -34.4 & 34.5 & -30.5 & 14.2 & -21.2 & 25.7 & -27.8 & 37.4 & -34.9 \\
\hline$\omega \mathrm{B} 97 \mathrm{X}-\mathrm{D}$ & $39.3-33.2$ & 37.9 & -34.0 & 38.5 & -32.5 & 32.1 & -30.0 & 33.8 & -31.5 & 37.1 & -34.4 \\
\hline CAM-B3LYP & $38.8-32.5$ & 37.6 & -33.3 & 38.2 & -31.5 & 32.3 & -29.3 & 33.7 & -30.9 & 37.0 & -33.8 \\
\hline LH07t-SVWN & $41.4-33.8$ & 40.9 & -33.0 & 40.3 & -34.6 & 35.6 & -31.5 & 37.3 & -32.9 & 39.7 & -35.0 \\
\hline cLH07t-SVWN & $41.6-34.0$ & 40.5 & -34.8 & 41.0 & -33.1 & 35.8 & -31.6 & 37.5 & -33.0 & 39.8 & -35.1 \\
\hline LH12ct-SsirPW92 & $41.5-34.1$ & 40.4 & -35.0 & 41.2 & -33.4 & 35.6 & -31.7 & 37.4 & -33.2 & 39.7 & -35.3 \\
\hline cLH12ct-SsirPW92 & $41.8-34.3$ & 40.5 & -35.0 & 41.0 & -33.1 & 35.8 & -31.6 & 37.5 & -33.0 & 39.8 & -35.4 \\
\hline LH14t-calPBE & $42.0-34.1$ & 41.0 & -34.9 & 41.3 & -33.2 & 36.0 & -31.6 & 37.9 & -33.1 & 40.3 & -35.2 \\
\hline cLH14-calPBE & $42.0-34.1$ & 40.9 & -34.9 & 41.3 & -33.5 & 35.8 & -31.8 & 37.6 & -33.2 & 40.2 & -35.2 \\
\hline LH20t & $43.2-34.8$ & 42.1 & -35.6 & 42.2 & -34.1 & 36.4 & -32.1 & 38.8 & -33.8 & 41.3 & -35.9 \\
\hline cLH20t & $42.6-34.6$ & 41.4 & -35.4 & 41.2 & -33.1 & 35.9 & -31.6 & 37.8 & -33.1 & 40.6 & -35.7 \\
\hline mPSTS & $42.6-34.2$ & 41.6 & -35.0 & 41.7 & -33.5 & 36.6 & -31.9 & 38.6 & -33.4 & 40.9 & -35.4 \\
\hline cmPSTS & $42.9-34.6$ & 41.8 & -35.4 & 41.9 & -33.6 & 36.8 & -32.1 & 38.8 & -33.7 & 41.1 & -35.7 \\
\hline LHJ14 & $43.0-34.3$ & 42.5 & -35.3 & 41.4 & -33.2 & 36.1 & -31.2 & 38.4 & -32.9 & 41.9 & -35.6 \\
\hline cLHJ14 & $41.4-33.7$ & 40.5 & -34.7 & 40.5 & -32.9 & 35.0 & -30.9 & 36.9 & -32.4 & 39.7 & -33.8 \\
\hline
\end{tabular}


[1] Suellen, C.; Freitas, R. G.; Loos, P.-F.; Jacquemin, D. Cross-Comparisons between Experiment, TD-DFT, CC, and ADC for Transition Energies. J. Chem. Theory Comput. 2019, 15, 4581-4590.

[2] Loos, P.-F.; Jacquemin, D. Chemically Accurate 0-0 Energies with Not-so-Accurate Excited State Geometries. J. Chem. Theory Comput. 2019, 15, 2481-2491.

[3] Dunning, T. H. Gaussian basis sets for use in correlated molecular calculations. I. The atoms boron through neon and hydrogen. J. Chem. Phys. 1989, 90, 1007-1023.

[4] Kendall, R. A.; Dunning, T. H.; Harrison, R. J. Electron affinities of the first-row atoms revisited. Systematic basis sets and wave functions. J. Chem. Phys. 1992, 96, 6796-6806.

[5] Woon, D. E.; Dunning, T. H. Gaussian basis sets for use in correlated molecular calculations. III. The atoms aluminum through argon. J. Chem. Phys. 1993, 98, 1358-1371.

[6] Furche, F.; Krull, B. T.; Nguyen, B. D.; Kwon, J. Accelerating molecular property calculations with nonorthonormal Krylov space methods. J. Chem. Phys. 2016, 144, 174105.

[7] Treutler, O.; Ahlrichs, R. Efficient molecular numerical integration schemes. J. Chem. Phys. 1995, 102, 346-354.

[8] Bates, J. E.; Furche, F. Harnessing the meta-generalized gradient approximation for time-dependent density functional theory. J. Chem. Phys. 2012, 137, 164105.

[9] Note that we have removed the incorrect extra factor of 2 for the antisymmetric part in the current-density response.

[10] Ahlrichs, R.; Bär, M.; Häser, M.; Horn, H.; Kölmel, C. Electronic structure calculations on workstation computers: The program system turbomole. Chem. Phys. Lett. 1989, 162, 165-169.

[11] Furche, F.; Ahlrichs, R.; Hättig, C.; Klopper, W.; Sierka, M.; Weigend, F. Turbomole. Wiley Interdiscip. Rev.: Comput. Mol. Sci. 2014, 4, 91-100.

[12] Balasubramani, S. G. et al. TURBOMOLE: Modular program suite for ab initio quantum-chemical and condensed-matter simulations. J. Chem. Phys. 2020, 152, 184107.

[13] Developers' version of TURBOMOLE V7.5.1 2021, a development of University of Karlsruhe and Forschungszentrum Karlsruhe GmbH, 1989-2007, TURBOMOLE GmbH, since 2007; available from https : //www . turbomole . org (retrieved November 22, 2020).

[14] Furche, F.; Rappoport, D. In Computational Photochemistry; Olivucci, M., Ed.; Theoretical and Computational Chemistry; Elsevier: Amsterdam, The Netherlands, 2005; Vol. 16; Chapter III, pp 93-128.

[15] Weigend, F.; Ahlrichs, R. Balanced basis sets of split valence, triple zeta valence and quadruple zeta valence quality for $\mathrm{H}$ to Rn: Design and assessment of accuracy. Phys. Chem. Chem. Phys. 2005, 7, 3297-3305.

[16] Holt, S. L.; Ballhausen, C. J. Low temperature absorption spectra of KMnO4 in KClo4. Theor. Chim. Acta 1967, 7, 313-320.

[17] McDiarmid, R. Assignments in the ultraviolet spectra of $\mathrm{MoF}_{6}$ and $\mathrm{WF}_{6}$. J. Chem. Phys. 1974, 61, 3333-3339.

[18] Houmøller, J.; Kaufman, S. H.; Støchkel, K.; Tribedi, L. C.; Brøndsted Nielsen, S.; Weber, J. M. On the Photoabsorption by Permanganate Ions in Vacuo and the Role of a Single Water Molecule. New Experimental Benchmarks for Electronic Structure Theory. ChemPhysChem 2013, 14, 1133-1137.

[19] Lever, A. B. P.; Ozin, G. A.; Hanlan, A. J. L.; Power, W. J.; Gray, H. B. Electronic absorption spectra of metal tetracarbonyls. Inorg. Chem. 1979, 18, 2088-2090.

[20] Maestri, M.; Sandrini, D.; Balzani, V.; Maeder, U.; Von Zelewsky, A. Absorption spectra, electrochemical behavior, luminescence spectra, and excited-state lifetimes of mixed-ligand ortho-metalated rhodium(III) complexes. Inorg. Chem. 1987, $26,1323-1327$.

[21] Kirketerp, M.-B. S.; Nielsen, S. B. Absorption spectrum of isolated tris(2,2'-bipyridine)ruthenium(II) dications in vacuo. Int. J. Mass Spectrom. 2010, 297, 63-66.

[22] Güdel, H. U.; Ballhausen, C. J. Low temperature absorption spectra of $\mathrm{TcO}_{4}^{-}$and $\mathrm{ReO}_{4}^{-}$in $\mathrm{KClO}_{4}$. Theor. Chim. Acta 1972 , $25,331-337$.

[23] Sekino, H.; Kobayashi, H. A screened potential molecular-orbital calculation of the $\pi$-electron systems of metalloporphin, metallochlorin, and metallobacteriochlorin. J. Chem. Phys. 1987, 86, 5045-5052.

[24] van Setten, M. J.; Weigend, F.; Evers, F. The GW-Method for Quantum Chemistry Applications: Theory and Implementation. J. Chem. Theory Comput. 2013, 9, 232-246.

[25] van Setten, M. J.; Caruso, F.; Sharifzadeh, S.; Ren, X.; Scheffler, M.; Liu, F.; Lischner, J.; Lin, L.; Deslippe, J. R.; Louie, S. G.; Yang, C.; Weigend, F.; Neaton, J. B.; Evers, F.; Rinke, P. GW100: Benchmarking G0W0 for Molecular Systems. J. Chem. Theory Comput. 2015, $11,5665-5687$.

[26] Krause, K.; Harding, M. E.; Klopper, W. Coupled-cluster reference values for the GW27 and GW100 test sets for the assessment of GW methods. Mol. Phys. 2015, 113, 1952-1960.

[27] Schäfer, A.; Huber, C.; Ahlrichs, R. Fully optimized contracted Gaussian basis sets of triple zeta valence quality for atoms Li to Kr. J. Chem. Phys. 1994, 100, 5829-5835.

[28] Eichkorn, K.; Treutler, O.; Öhm, H.; Häser, M.; Ahlrichs, R. Auxiliary Basis Sets to Approximate Coulomb Potentials. Chem. Phys. Lett. 1995, 242, 283-290.

[29] Eichkorn, K.; Weigend, F.; Treutler, O.; Ahlrichs, R. Auxiliary basis sets for main row atoms and transition metals and their use to approximate Coulomb potentials. Theor. Chem. Acc. 1997, 97, 119-124.

[30] Weigend, F. Accurate Coulomb-fitting basis sets for H to Rn. Phys. Chem. Chem. Phys. 2006, 8, 1057-1065.

[31] Weigend, F.; Kattannek, M.; Ahlrichs, R. Approximated electron repulsion integrals: Cholesky decomposition versus resolution of the identity methods. J. Chem. Phys. 2009, 130, 164106.

[32] Weigend, F.; Häser, M.; Patzelt, H.; Ahlrichs, R. RI-MP2: optimized auxiliary basis sets and demonstration of efficiency. Chem. Phys. Lett. 1998, 294, 143-152.

[33] Hellweg, A.; Hättig, C.; Höfener, S.; Klopper, W. Optimized accurate auxiliary basis sets for RI-MP2 and RI-CC2 calculations for the 
atoms $\mathrm{Rb}$ to $\mathrm{Rn}$. Theor. Chem. Acc. 2007, 117, 587-597.

[34] Becke, A. D. Density-functional exchange-energy approximation with correct asymptotic behavior. Phys. Rev. A 1988, 38, 3098-3100.

[35] Perdew, J. P. Density-functional approximation for the correlation energy of the inhomogeneous electron gas. Phys. Rev. B 1986, 33, 8822-8824.

[36] Perdew, J. P.; Burke, K.; Ernzerhof, M. Generalized Gradient Approximation Made Simple. Phys. Rev. Lett. 1996, 77, $3865-3868$.

[37] Kaplan, F.; Weigend, F.; Evers, F.; van Setten, M. J. Off-Diagonal Self-Energy Terms and Partially Self-Consistency in GW Calculations for Single Molecules: Efficient Implementation and Quantitative Effects on Ionization Potentials. J. Chem. Theory Comput. 2015, 11, 5152-5160.

[38] Kaplan, F.; Harding, M. E.; Seiler, C.; Weigend, F.; Evers, F.; van Setten, M. J. Quasi-Particle Self-Consistent GW for Molecules. J. Chem. Theory Comput. 2016, 12, 2528-2541.

[39] Holzer, C.; Klopper, W. Ionized, electron-attached, and excited states of molecular systems with spin-orbit coupling: Two-component GW and Bethe-Salpeter implementations. J. Chem. Phys. 2019, 150, 204116.

[40] Holzer, C.; Teale, A. M.; Hampe, F.; Stopkowicz, S.; Helgaker, T.; Klopper, W. GW quasiparticle energies of atoms in strong magnetic fields. J. Chem. Phys. 2019, 150, 214112.

[41] Holzer, C.; Teale, A. M.; Hampe, F.; Stopkowicz, S.; Helgaker, T.; Klopper, W. Erratum: "GW quasiparticle energies of atoms in strong magnetic fields" [J. Chem. Phys. 150, 214112 (2019)]. J. Chem. Phys. 2019, 151, 069902.

[42] Kotani, T.; Van Schilfgaarde, M.; Faleev, S. V. Quasiparticle self-consistent GW method: A basis for the independent-particle approximation. Phys. Rev. B 2007, 76, 165106.

[43] Häser, M.; Ahlrichs, R.; Baron, H. P.; Weis, P.; Horn, H. Direct computation of second-order scf properties of large molecules on workstation computers with an application to large carbon clusters. Theor. Chim. Acta 1992, 83, 455-470.

[44] Huniar, U. Berechnung der chemischen Verschiebung der NMR mit Methoden der Dichtefunktionaltheorie (DFT). Diploma Thesis, University of Karlsruhe (TH), Germany, 1999.

[45] Reiter, K.; Mack, F.; Weigend, F. Calculation of Magnetic Shielding Constants with meta-GGA Functionals Employing the MultipoleAccelerated Resolution of the Identity: Implementation and Assessment of Accuracy and Efficiency. J. Chem.Theory Comput. 2018, 14, 191-197.

[46] Jusélius, J.; Sundholm, D.; Gauss, J. Calculation of current densities using gauge-including atomic orbitals. J. Chem. Phys. 2004, 121, 3952-3963.

[47] Fliegl, H.; Taubert, S.; Lehtonen, O.; Sundholm, D. The gauge including magnetically induced current method. Phys. Chem. Chem. Phys. 2011, 13, 20500-20518.

[48] Sundholm, D.; Fliegl, H.; Berger, R. J. F. Calculations of magnetically induced current densities: Theory and applications. Wiley Interdiscip. Rev.: Comput. Mol. Sci. 2016, 6, 639-678.

[49] GIMIC. Version 2.1.4 (merge 6c574ed, 2020), available from https ://github.com/qmcurrents/gimic (retrieved March 28, 2021).

[50] Lehtola, S.; Dimitrova, M.; Fliegl, H.; Sundholm, D. Benchmarking Magnetizabilities with Recent Density Functionals. J. Chem. Theory Comput. 2021, 17, 1457-1468.

[51] Ruud, K.; Helgaker, T.; Bak, K. L.; Jørgensen, P.; Jensen, H. J. A. Hartree-Fock limit magnetizabilities from London orbitals. J. Chem. Phys. 1993, 99, 3847-3859.

[52] Ruud, K.; Helgaker, T.; Jørgensen, P.; Bak, K. L. Theoretical calculations of the magnetizability of some small fluorine-containing molecules using London atomic orbitals. Chem. Phys. Lett. 1994, 223, 12-18.

[53] Ruud, K.; Skaane, H.; Helgaker, T.; Bak, K. L.; Joergensen, P. Magnetizability of hydrocarbons. J. Am. Chem. Soc. 1994, 116, 1013510140.

[54] Hait, D.; Head-Gordon, M. How accurate are static polarizability predictions from density functional theory? An assessment over 132 species at equilibrium geometry. Phys. Chem. Chem. Phys. 2018, 20, 19800-19810.

[55] Haynes, W. M., Ed. CRC Handbook of Chemistry and Physics; CRC Press: Boca Ratón, USA, 2014.

[56] Lutnæs, O. B.; Teale, A. M.; Helgaker, T.; Tozer, D. J.; Ruud, K.; Gauss, J. Benchmarking density-functional-theory calculations of rotational g tensors and magnetizabilities using accurate coupled-cluster calculations. J. Chem. Phys. 2009, 131, 144104.

[57] Peterson, K. E.; Dunning, T. H. Accurate correlation consistent basis sets for molecular core-valence correlation effects: The second row atoms Al-Ar, and the first row atoms B-Ne revisited. J. Chem. Phys. 2002, 117, 10548-10560.

[58] Woon, D. E.; Dunning, T. H. Gaussian basis sets for use in correlated molecular calculations. V. Core-valence basis sets for boron through neon. J. Chem. Phys. 1995, 103, 4572-4585.

[59] Prascher, B. P.; Woon, D. E.; Peterson, K. A.; Dunning, T. H.; Wilson, A. K. Gaussian basis sets for use in correlated molecular calculations. VII. Valence, core-valence, and scalar relativistic basis sets for $\mathrm{Li}, \mathrm{Be}, \mathrm{Na}$, and $\mathrm{Mg}$. Theor. Chem. Acc. 2011, 128, 69-82.

[60] ccRepo of the Hill group, http://www.grant-hill.group.shef .ac.uk/ccrepo/(retrieved January 24, 2021).

[61] Benedikt, U.; Auer, A. A.; Jensen, F. Optimization of augmentation functions for correlated calculations of spin-spin coupling constants and related properties. J. Chem. Phys. 2008, 129, 064111.

[62] Pritchard, B. P.; Altarawy, D.; Didier, B.; Gibson, T. D.; Windus, T. L. New Basis Set Exchange: An Open, Up-to-Date Resource for the Molecular Sciences Community. J. Chem. Inf. Model. 2019, 59, 4814-4820.

[63] Basis set exchange library ver2, BSE Library v0.8.12, https ://www .basissetexchange.org/(retrieved March 5, 2020).

[64] Faber, R.; Sauer, S. P. A.; Gauss, J. Importance of Triples Contributions to NMR Spin-Spin Coupling Constants Computed at the CC3 and CCSDT Levels. J. Chem. Theory Comput. 2017, 13, 696-709.

[65] Ramsey, N. F. Electron Coupled Interactions between Nuclear Spins in Molecules. Phys. Rev. 1953, 91, 303-307.

[66] Mack, F.; Schattenberg, C. J.; Kaupp, M.; Weigend, F. Nuclear Spin-Spin Couplings: Efficient Evaluation of Exact Exchange and Extension to Local Hybrid Functionals. J. Phys. Chem. A 2020, 124, 8529-8539.

[67] Flaig, D.; Maurer, M.; Hanni, M.; Braunger, K.; Kick, L.; Thubauville, M.; Ochsenfeld, C. Benchmarking Hydrogen and Carbon NMR Chemical Shifts at HF, DFT, and MP2 Levels. J. Chem. Theory Comput. 2014, 10, 572-578. 
[68] Keal, T. W.; Tozer, D. J. A semiempirical generalized gradient approximation exchange-correlation functional. J. Chem. Phys. 2004, 121, 5654-5660.

[69] Ekström, U.; Visscher, L.; Bast, R.; Thorvaldsen, A. J.; Ruud, K. Arbitrary-Order Density Functional Response Theory from Automatic Differentiation. J. Chem. Theory Comput. 2010, 6, 1971-1980.

[70] Tao, J.; Perdew, J. P.; Staroverov, V. N.; Scuseria, G. E. Climbing the Density Functional Ladder: Nonempirical Meta-Generalized Gradient Approximation Designed for Molecules and Solids. Phys. Rev. Lett. 2003, 91, 146401.

[71] Lee, C.; Yang, W.; Parr, R. G. Development of the Colle-Salvetti correlation-energy formula into a functional of the electron density. Phys. Rev. B 1988, 37, 785-789.

[72] Becke, A. D. A new mixing of Hartree-Fock and local density-functional theories. J. Chem. Phys. 1993, 98, $1372-1377$.

[73] Becke, A. D. Density-functional thermochemistry. III. The role of exact exchange. J. Chem. Phys. 1993, 98, 5648-5652.

[74] Stephens, P. J.; Devlin, F. J.; Chabalowski, C. F.; Frisch, M. J. Ab Initio Calculation of Vibrational Absorption and Circular Dichroism Spectra Using Density Functional Force Fields. J. Phys. Chem. 1994, 98, 11623-11627.

[75] Adamo, C.; Barone, V. Toward reliable density functional methods without adjustable parameters: The PBE0 model. J. Chem. Phys. 1999, 110, 6158-6170.

[76] Staroverov, V. N.; Scuseria, G. E.; Tao, J.; Perdew, J. P. Comparative assessment of a new nonempirical density functional: Molecules and hydrogen-bonded complexes. J. Chem. Phys. 2003, 119, 12129-12137.

[77] Vydrov, O. A.; Scuseria, G. E. Assessment of a long-range corrected hybrid functional. J. Chem. Phys. 2006, $125,234109$.

[78] Marques, M. A. L.; Oliveira, M. J. T.; Burnus, T. Libxc: A library of exchange and correlation functionals for density functional theory. Comput. Phys. Commun. 2012, 183, 2272-2281.

[79] Lehtola, S.; Steigemann, C.; Oliveira, M. J. T.; Marques, M. A. L. Recent developments in libxc - A comprehensive library of functionals for density functional theory. SoftwareX 2018, 7, 1-5.

[80] Chai, J.-D.; Head-Gordon, M. Long-range corrected hybrid density functionals with damped atom-atom dispersion corrections. Phys. Chem. Chem. Phys. 2008, 10, 6615-6620.

[81] Yanai, T.; Tew, D. P.; Handy, N. C. A new hybrid exchange-correlation functional using the Coulomb-attenuating method (CAMB3LYP). Chem. Phys. Lett. 2004, 393, 51-57.

[82] Jin, Y.; Bartlett, R. J. The QTP family of consistent functionals and potentials in Kohn-Sham density functional theory. J. Chem. Phys. 2016, $145,034107$.

[83] Haiduke, R. L. A.; Bartlett, R. J. Communication: Can excitation energies be obtained from orbital energies in a correlated orbital theory? J. Chem. Phys. 2018, 149, 131101.

[84] Bahmann, H.; Rodenberg, A.; Arbuznikov, A. V.; Kaupp, M. A thermochemically competitive local hybrid functional without gradient corrections. J. Chem. Phys. 2007, 126, 011103.

[85] Arbuznikov, A. V.; Kaupp, M. Importance of the correlation contribution for local hybrid functionals: Range separation and selfinteraction corrections. J. Chem. Phys. 2012, 136, 014111.

[86] Arbuznikov, A. V.; Kaupp, M. Towards improved local hybrid functionals by calibration of exchange-energy densities. J. Chem. Phys. 2014, 141, 204101.

[87] Haasler, M.; Maier, T. M.; Grotjahn, R.; Gückel, S.; Arbuznikov, A. V.; Kaupp, M. A Local Hybrid Functional with Wide Applicability and Good Balance between (De)Localization and Left-Right Correlation. J. Chem. Theory Comput. 2020, 16, $5645-5657$.

[88] Johnson, E. R. Local-hybrid functional based on the correlation length. J. Chem. Phys. 2014, 141, 124120.

[89] Plessow, P.; Weigend, F. Seminumerical calculation of the Hartree-Fock exchange matrix: Application to two-component procedures and efficient evaluation of local hybrid density functionals. J. Comput. Chem. 2012, 33, 810-816.

[90] Schattenberg, C. J.; Reiter, K.; Weigend, F.; Kaupp, M. An Efficient Coupled-Perturbed Kohn-Sham Implementation of NMR Chemical Shift Computations with Local Hybrid Functionals and Gauge-Including Atomic Orbitals. J. Chem. Theory Comput. 2020, 16, 931-943.

[91] Holzer, C. An improved seminumerical Coulomb and exchange algorithm for properties and excited states in modern density functional theory. J. Chem. Phys. 2020, 153, 184115.

[92] Stoychev, G. L.; Auer, A. A.; Izsák, R.; Neese, F. Self-Consistent Field Calculation of Nuclear Magnetic Resonance Chemical Shielding Constants Using Gauge-Including Atomic Orbitals and Approximate Two-Electron Integrals. J. Chem. Theory Comput. 2018, 14, 619637.

[93] Jensen, F. Segmented Contracted Basis Sets Optimized for Nuclear Magnetic Shielding. J. Chem. Theory Comput. 2015, 11, $132-138$.

[94] Peng, D.; Reiher, M. Local relativistic exact decoupling. J. Chem. Phys. 2012, 136, 244108.

[95] Peng, D.; Middendorf, N.; Weigend, F.; Reiher, M. An efficient implementation of two-component relativistic exact-decoupling methods for large molecules. J. Chem. Phys. 2013, 138, 184105.

[96] Franzke, Y. J.; Middendorf, N.; Weigend, F. Efficient implementation of one- and two-component analytical energy gradients in exact two-component theory. J. Chem. Phys. 2018, 148, 104410.

[97] Klamt, A.; Schüürmann, G. COSMO: a new approach to dielectric screening in solvents with explicit expressions for the screening energy and its gradient. J. Chem. Soc., Perkin Trans. 2 1993, 799-805.

[98] Schäfer, A.; Klamt, A.; Sattel, D.; Lohrenz, J. C. W.; Eckert, F. COSMO Implementation in TURBOMOLE: Extension of an efficient quantum chemical code towards liquid systems. Phys. Chem. Chem. Phys. 2000, 2, 2187-2193.

[99] Franzke, Y. J.; Treß, R.; Pazdera, T. M.; Weigend, F. Error-consistent segmented contracted all-electron relativistic basis sets of doubleand triple-zeta quality for NMR shielding constants. Phys. Chem. Chem. Phys. 2019, 21, 16658-16664.

[100] Franzke, Y. J.; Weigend, F. NMR Shielding Tensors and Chemical Shifts in Scalar-Relativistic Local Exact Two-Component Theory. J. Chem. Theory Comput. 2019, 15, 1028-1043.

[101] Visscher, L.; Dyall, K. G. Dirac-Fock atomic electronic structure calculations using different nuclear charge distributions. At. Data Nucl. Data Tables 1997, 67, 207-224.

[102] Figgis, B. N.; Kidd, R. G.; Nyholm, R. S. Oxygen-17 nuclear magnetic resonance of inorganic compounds. Proc. Royal Soc. Lond. A 
1962, 269, 469-480.

[103] Kaupp, M.; Malkin, V. G.; Malkina, O. L.; Salahub, D. R. Scalar Relativistic Effects on 17O NMR Chemical Shifts in Transition-Metal Oxo Complexes. An ab Initio ECP/DFT Study. J. Am. Chem. Soc. 1995, 117, 1851-1852.

[104] Kaupp, M.; Malkin, V. G.; Malkina, O. L.; Salahub, D. R. Erratum: "Scalar Relativistic Effects on 17O NMR Chemical Shifts in Transition-Metal Oxo Complexes. An ab Initio ECP/DFT Study”. J. Am. Chem. Soc. 1995, 117, 8492-8492.

[105] Cheng, L.; Gauss, J.; Stanton, J. F. Treatment of scalar-relativistic effects on nuclear magnetic shieldings using a spin-free exact-twocomponent approach. J. Chem. Phys. 2013, 139, 054105.

[106] Gawrilow, M.; Beckers, H.; Riedel, S.; Cheng, L. Matrix-Isolation and Quantum-Chemical Analysis of the $\mathrm{C}_{3 v} \mathrm{Conformer}_{\mathrm{N}} \mathrm{XeF}_{6}$, $\mathrm{XeOF}_{4}$, and Their Acetonitrile Adducts. J. Chem. Phys. A 2018, 122, 119-129.

[107] Gerken, M.; Hazendonk, P.; Nieboer, J.; Schrobilgen, G. J. NMR spectroscopic study of xenon fluorides in the gas phase and of XeF2 in the solid state. J. Fluor. Chem. 2004, 125, 1163-1168.

[108] Jameson, C. In Multinuclear NMR; Mason, J., Ed.; Springer US: New York, USA, 1987; Chapter 18, pp $463-477$.

[109] Roos, B. O.; Lindh, R.; Malmqvist, P.-Å; Veryazov, V.; Widmark, P.-O. Main Group Atoms and Dimers Studied with a New Relativistic ANO Basis Set. J. Phys. Chem. A 2004, 108, 2851-2858.

[110] Rodriguez-Fortea, A.; Alemany, P.; Ziegler, T. Density Functional Calculations of NMR Chemical Shifts with the Inclusion of SpinOrbit Coupling in Tungsten and Lead Compounds. J. Phys. Chem. A 1999, 103, 8288-8294.

[111] Banck, J.; Schwenk, A. ${ }^{183}$ Tungsten NMR studies. Z. Phys. B 1975, 20, 75-80.

[112] Gheller, S. F.; Hambley, T. W.; Rodgers, J. R.; Brownlee, R. T. C.; O’Connor, M. J.; Snow, M. R.; Wedd, A. G. Synthesis and characterization of complexes of thiomolybdates and thiotungstates with copper(I) and silver(I) cyanides, including molybdenum-95 and tungsten-183 NMR properties and the crystal and molecular structures of $\left(\mathrm{n}-\mathrm{Pr}_{4} \mathrm{~N}\right)_{2}\left[(\mathrm{CN}) \mathrm{CuS}_{2} \mathrm{MoS}_{2}\right],\left(\mathrm{n}-\mathrm{Pr}_{4} \mathrm{~N}\right)_{2}\left[(\mathrm{CN}) \mathrm{AgS} \mathrm{SS}_{2}\right]$, and $\left(\mathrm{Ph}_{4} \mathrm{As}\right)_{2}\left[(\mathrm{CN}) \mathrm{CuS}_{2} \mathrm{MoS}_{2} \mathrm{Cu}(\mathrm{CN})\right] \cdot \mathrm{H}_{2} \mathrm{O}$. Inorg. Chem. 1984, 23, 2519-2528.

[113] Keiter, R. L.; Velde, D. G. V. Direct observation of ${ }^{183} \mathrm{~W}$ NMR of W(CO) 6 and phosphine derivatives. J. Organomet. Chem. 1983, 258, C34-C36.

[114] Mann, B. In Annual Reports on NMR Spectroscopy; Webb, G., Ed.; Academic Press: San Diego, USA, 1991; Vol. 23 ; pp 141-207.

[115] GIMIC. Version 2.1.4 (merge 3a5f0eb, 2019), available from https://github.com/qmcurrents/gimic (retrieved November 26, 2020).

[116] Eulenstein, A. R.; Franzke, Y. J.; Lichtenberger, N.; Wilson, R. J.; Deubner, H. L.; Kraus, F.; Weigend, F.; Dehnen, S. Substantial $\pi$-aromaticity of the anionic heavy-metal cluster $\left[\mathrm{Th}_{\mathrm{B}} \mathrm{Bi}_{12}\right]^{4-}$. Nat. Chem. 2021, 13, 149-155.

[117] Cao, X.; Dolg, M.; Stoll, H. Valence basis sets for relativistic energy-consistent small-core actinide pseudopotentials. J. Chem. Phys. 2003, 118, 487-496.

[118] Metz, B.; Stoll, H.; Dolg, M. Small-core multiconfiguration-Dirac-Hartree-Fock-adjusted pseudopotentials for post-d main group elements: Application to $\mathrm{PbH}$ and PbO. J. Chem. Phys. 2000, 113, 2563-2569.

[119] Dyall, K. G. Relativistic and nonrelativistic finite nucleus optimized triple-zeta basis sets for the 4p, 5p and 6p elements. Theor. Chem. Acc. 2002, 108, 335-340.

[120] Dyall, K. G. Relativistic double-zeta, triple-zeta, and quadruple-zeta basis sets for the actinides Ac-Lr. Theor. Chem. Acc. 2007, 117, 491-500.

[121] Basis sets available from the Dirac program web site, http://dirac.chem.sdu.dk (retrieved December 4, 2020). Note that we have deleted the inner-most g-function of the $6 \mathrm{~d} / 7 \mathrm{~s}$ correlating set of Th.

[122] Martins, L.; Jorge, F.; Machado, S. All-electron segmented contraction basis sets of triple zeta valence quality for the fifth-row elements. Mol. Phys. 2015, 113, 3578-3586.

[123] de Oliveira, A.; Campos, C.; Jorge, F.; Ferreira, I.; Fantin, P. All-electron triple zeta basis sets for the actinides. Comput. Theor. Chem. 2018, 1135, 28-33.

[124] Franzke, Y. J.; Spiske, L.; Pollak, P.; Weigend, F. Segmented Contracted Error-Consistent Basis Sets of Quadruple- $\zeta$ Valence Quality for One- and Two-Component Relativistic All-Electron Calculations. J. Chem. Theory Comput. 2020, 16, 5658-5674.

[125] Schleyer, P. v. R.; Maerker, C.; Dransfeld, A.; Jiao, H.; Hommes, N. J. R. v. E. Nucleus-independent chemical shifts: A simple and efficient aromaticity probe. J. Am. Chem. Soc. 1996, 118, 6317-6318.

[126] von Schleyer, P. R.; Jiao, H. What is aromaticity? Pure \& Appl. Chem. 1996, 68, 209-218.

[127] Chen, Z.; Wannere, C. S.; Corminboeuf, C.; Puchta, R.; Schleyer, P. v. R. Nucleus-independent chemical shifts (NICS) as an aromaticity criterion. Chem. Rev. 2005, 105, 3842-3888.

[128] Gershoni-Poranne, R.; Stanger, A. Magnetic criteria of aromaticity. Chem. Soc. Rev. 2015, 44, 6597-6615. 